TRANSACTIONS OF THE

AMERICAN MATHEMATICAL SOCIETY

Volume 359, Number 8, August 2007, Pages 3791-3858

S 0002-9947(07)04225-0

Article electronically published on March 20, 2007

\title{
EXTENSIONS OF $p$-LOCAL FINITE GROUPS
}

\author{
C. BROTO, N. CASTELlANA, J. GRODAL, R. LEVI, AND B. OLIVER
}

\begin{abstract}
A $p$-local finite group consists of a finite $p$-group $S$, together with a pair of categories which encode "conjugacy" relations among subgroups of $S$, and which are modelled on the fusion in a Sylow $p$-subgroup of a finite group. It contains enough information to define a classifying space which has many of the same properties as $p$-completed classifying spaces of finite groups. In this paper, we study and classify extensions of $p$-local finite groups, and also compute the fundamental group of the classifying space of a $p$-local finite group.
\end{abstract}

A $p$-local finite group consists of a finite $p$-group $S$, together with a pair of categories $(\mathcal{F}, \mathcal{L})$, of which $\mathcal{F}$ is modeled on the conjugacy (or fusion) in a Sylow subgroup of a finite group. The category $\mathcal{L}$ is essentially an extension of $\mathcal{F}$ and contains just enough extra information so that its $p$-completed nerve has many of the same properties as $p$-completed classifying spaces of finite groups. We recall the precise definitions of these objects in Section 1, and refer to BLO2] and [5A1] for motivation for their study.

In this paper, we study extensions of saturated fusion systems and of $p$-local finite groups. This is in continuation of our more general program of trying to understand to what extent properties of finite groups can be extended to properties of $p$-local finite groups, and to shed light on the question of how many (exotic) $p$-local finite groups there are. While we do not get a completely general theory of extensions of one $p$-local finite group by another, we do show how certain types of extensions can be described in a manner very similar to the situation for finite groups.

From the point of view of group theory, developing an extension theory for $p$ local finite groups is related to the question of to what extent the extension problem for groups is a local problem, i.e., a problem purely described in terms of a Sylow $p$-subgroup and conjugacy relations inside it. In complete generality this is not the case. For example, strongly closed subgroups of a Sylow $p$-subgroup $S$ of $G$ need not correspond to normal subgroups of $G$. However, special cases where this does happen include the case of existence of $p$-group quotients (the focal subgroup theorems; see [Go, §§7.3-7.4]) and central subgroups (described via the $Z^{*}$-theorem of Glauberman Gl]).

Received by the editors July 11, 2005.

2000 Mathematics Subject Classification. Primary 55R35; Secondary 55R40, 20D20.

Key words and phrases. Classifying space, $p$-completion, finite groups, fusion.

The first author was partially supported by MCYT grant BFM2001-2035.

The second author was partially supported by MCYT grant BFM2001-2035.

The third author was partially supported by NSF grants DMS-0104318 and DMS-0354633.

The fourth author was partially supported by EPSRC grant GR/M7831.

The fifth author was partially supported by UMR 7539 of the CNRS.

(C)2007 American Mathematical Society 
From the point of view of homotopy theory, one of the problems which comes up when looking for a general theory of extensions of $p$-local finite groups is that while an extension of groups $1 \rightarrow K \rightarrow \Gamma \rightarrow G \rightarrow 1$ always induces a (homotopy) fibration sequence of classifying spaces, it does not in general induce a fibration sequence of $p$-completed classifying spaces. Two cases where this does happen are those where $G$ is a $p$-group, and where the extension is central. In both of these cases, $B H_{p}^{\wedge}$ is the homotopy fiber of the map $B \Gamma_{p}^{\wedge} \longrightarrow B G_{p}^{\wedge}$. Thus, also from the point of view of homotopy theory, it is natural to study extensions of $p$-local finite groups with a $p$-group quotient, and to study central extensions of $p$-local finite groups. The third case we study is that of extensions with a quotient of order prime to $p$; and the case of $p$ and $p^{\prime}$-group quotients to some extent unify to give a theory of extensions with $p$-solvable quotient. Recall in this connection that by a previous result of ours [5A1, Proposition C], solvable $p$-local finite groups all come from $p$-solvable groups. In all three of these situations, we develop a theory of extensions which parallels the situation for finite groups.

We now describe the contents of this paper in more detail, stating simplified versions of our main results on extensions. Stronger and more precise versions of some of these theorems will be stated and proven later.

In Section 3, we construct a very general theory of fusion subsystems (Proposition 3.8) and linking subsystems (Theorem 3.9) with quotient a $p$-group or a group of order prime to $p$. As a result we get the following theorem (Corollary [3.10), which for a $p$-local finite group $(S, \mathcal{F}, \mathcal{L})$, describes a correspondence between covering spaces of the geometric realization $|\mathcal{L}|$ and certain $p$-local finite subgroups of $(S, \mathcal{F}, \mathcal{L})$.

Theorem A. Suppose that $(S, \mathcal{F}, \mathcal{L})$ is a p-local finite group. Then there is a normal subgroup $H \triangleleft \pi_{1}(|\mathcal{L}|)$ which is minimal among all those whose quotient is finite and p-solvable. Any covering space of the geometric realization $|\mathcal{L}|$ whose fundamental group contains $H$ is homotopy equivalent to $\left|\mathcal{L}^{\prime}\right|$ for some $p$-local finite group $\left(S^{\prime}, \mathcal{F}^{\prime}, \mathcal{L}^{\prime}\right)$, where $S^{\prime} \leq S$ and $\mathcal{F}^{\prime} \subseteq \mathcal{F}$.

Moreover, the $p$-local finite group $\left(S^{\prime}, \mathcal{F}^{\prime}, \mathcal{L}^{\prime}\right)$ of Theorem $\mathrm{A}$ can be explicitly described in terms of $\mathcal{L}$, as we will explain in Section 3.

In order to use this theorem, it is useful to have ways of finding the finite $p$ solvable quotients of $\pi_{1}(|\mathcal{L}|)$. This can be done by iteration, using the next two theorems. In them, the maximal $p$-group quotient of $\pi_{1}(|\mathcal{L}|)$, and the maximal quotient of order prime to $p$, are described solely in terms of the fusion system $\mathcal{F}$.

When $G$ is an infinite group, we define $O^{p}(G)$ and $O^{p^{\prime}}(G)$ to be the intersection of all normal subgroups of $G$ of $p$-power index, or index prime to $p$, respectively. These clearly generalize the usual definitions for finite $G$ (but are not the only possible generalizations).

Theorem B (Hyperfocal subgroup theorem for $p$-local finite groups). For a $p$-local finite group $(S, \mathcal{F}, \mathcal{L})$, the natural homomorphism

$$
S \longrightarrow \pi_{1}(|\mathcal{L}|) / O^{p}\left(\pi_{1}(|\mathcal{L}|)\right) \cong \pi_{1}\left(|\mathcal{L}|_{p}^{\wedge}\right)
$$

is surjective, with kernel equal to

$$
O_{\mathcal{F}}^{p}(S) \stackrel{\text { def }}{=}\left\langle g^{-1} \alpha(g) \in S \mid g \in P \leq S, \alpha \in O^{p}\left(\operatorname{Aut}_{\mathcal{F}}(P)\right)\right\rangle .
$$

When $G$ is a finite group with Sylow $p$-subgroup $S$, then we have $\pi_{1}\left(\left|\mathcal{L}_{p}(G)\right|_{p}^{\wedge}\right) \cong$ $\pi_{1}\left(B G_{p}^{\wedge}\right) \cong G / O^{p}(G)$, and Theorem B becomes the hyperfocal subgroup theorem 
of Puig [Pu2, §1.1], which describes $S \cap O^{p}(G)$ in terms of $G$ fusion in $S$. This in turn was motivated by the classical focal subgroup theorem (cf. Go, Theorem 7.3.4]), which describes $S \cap[G, G]$ in a similar way.

For any saturated fusion system $\mathcal{F}$ over a $p$-group $S$, we let $O_{*}^{p^{\prime}}(\mathcal{F}) \subseteq \mathcal{F}$ be the smallest fusion subsystem of $\mathcal{F}$ (in the sense of Definition 1.1) which contains all automorphism groups $O^{p^{\prime}}\left(\operatorname{Aut}_{\mathcal{F}}(P)\right)$ for $P \leq S$. Equivalently, $O_{*}^{p^{\prime}}(\mathcal{F})$ is the smallest subcategory of $\mathcal{F}$ with the same objects, and which contains all restrictions of all automorphisms in $\mathcal{F}$ of $p$-power order. This subcategory is needed in the statement of the next theorem.

Theorem C. For a p-local finite group $(S, \mathcal{F}, \mathcal{L})$, the natural map

$$
\operatorname{Out}_{\mathcal{F}}(S) \longrightarrow \pi_{1}(|\mathcal{L}|) / O^{p^{\prime}}\left(\pi_{1}(|\mathcal{L}|)\right)
$$

is surjective, with kernel equal to

$$
\operatorname{Out}_{\mathcal{F}}^{0}(S) \stackrel{\text { def }}{=}\left\langle\alpha \in \operatorname{Out}_{\mathcal{F}}(S)|\alpha|_{P} \in \operatorname{Mor}_{O_{*}^{p^{\prime}}(\mathcal{F})}(P, S) \text {, some } \mathcal{F} \text {-centric } P \leq S\right\rangle .
$$

Theorem $\mathrm{C}$ is analogous to the result that for a finite group $G$ with Sylow $p$ subgroup $S$, the quotient $N_{G}(S) / S$ surjects onto $G / O^{p^{\prime}}(G)$. Theorem B is proved as Theorem 2.5, and Theorem $\mathrm{C}$ is proved as Theorem [5.5.

In fact, we give a purely algebraic description of these subsystems of " $p$-power index" or of "index prime to $p$ " (Definition 3.1), and then show in Sections 4.1 and 5.1 that they in fact all arise as finite covering spaces of $|\mathcal{L}|$ (see Theorems 4.4 and 5.5). Subsystems of fusion systems of index prime to $p$ were earlier studied by Puig Pu1, using somewhat different arguments and terminology. He showed that there is a minimal such subsystem (which he calls the "adjoint" subsystem), and gave an algebraic construction of intermediate ones. Much later, subsystems of $p$-power index were also studied by Puig [ $\mathrm{Pu}$ ], independently of us, under the assumption that there is an associated linking system.

Afterwards, in Sections 4.2 and 5.2, we establish converses to these concerning the extensions of a $p$-local finite group, which include the following theorem.

Theorem D. Let $(S, \mathcal{F}, \mathcal{L})$ be a p-local finite group. Suppose we are given a fibration sequence $|\mathcal{L}|_{p}^{\wedge} \rightarrow E \rightarrow B G$, where $G$ is a finite p-group or has order prime to p. Then there exists a p-local finite group $\left(S^{\prime}, \mathcal{F}^{\prime}, \mathcal{L}^{\prime}\right)$ containing $(S, \mathcal{F}, \mathcal{L})$ such that $\left|\mathcal{L}^{\prime}\right|_{p}^{\wedge} \simeq E_{p}^{\wedge}$.

This is shown as Theorems 4.7 and 5.8. Moreover, when $G$ is a $p$-group, we give in Theorem 4.7 an explicit algebraic construction of the $p$-local finite group $\left(S^{\prime}, \mathcal{F}^{\prime}, \mathcal{L}^{\prime}\right)$. In the setting of saturated fusion systems, extensions by finite groups of order prime to $p$ were earlier constructed by Puig in [Pu1, Theorem 2.17].

Finally, in Section 6, we develop the theory of central extensions of $p$-local finite groups. Our main results there (Theorems 6.8 and 6.13) give a more elaborate version of the following theorem. Here, the center of a $p$-local finite group $(S, \mathcal{F}, \mathcal{L})$ is defined to be the subgroup of elements $x \in Z(S)$ such that $\alpha(x)=x$ for all $\alpha \in \operatorname{Mor}\left(\mathcal{F}^{c}\right)$.

Theorem E. Suppose that $A$ is a central subgroup of a p-local finite group $(S, \mathcal{F}, \mathcal{L})$. Then there exists a canonical quotient $p$-local finite group $(S / A, \mathcal{F} / A, \mathcal{L} / A)$, and the canonical projection of $|\mathcal{L}|$ onto $|\mathcal{L} / A|$ is a principal fibration with fiber $B A$. 
Conversely, for any principal fibration $E \rightarrow|\mathcal{L}|$ with fiber $B A$, where $A$ is a finite abelian p-group, there exists a p-local finite group $(\widetilde{S}, \widetilde{\mathcal{F}}, \widetilde{\mathcal{L}})$ such that $|\widetilde{\mathcal{L}}| \simeq E$. Furthermore, this correspondence sets up a $1-1$ correspondence between central extensions of $\mathcal{L}$ by $A$ and elements in $H^{2}(|\mathcal{L}| ; A)$.

One motivation for this study was the question of whether extensions of $p$-local finite groups coming from finite groups can produce exotic $p$-local finite groups. In the case of central extensions, we are able to show that $(S, \mathcal{F}, \mathcal{L})$ comes from a finite group if and only if $(S / A, \mathcal{F} / A, \mathcal{L} / A)$ comes from a group (Corollary 6.14). For the other types of extensions studied in this paper, this is still an open question. We have so far failed to produce exotic examples in this way, and yet we have also been unable to show that exotic examples cannot occur. This question seems to be related to some rather subtle and interesting group theoretic issues relating local to global structure; see Corollary 4.8 for one partial result in this direction.

This paper builds on the earlier paper [5A1] by the same authors, and many of the results in that paper were originally motivated by this work on extensions.

The authors would like to thank the University of Aberdeen, Universitat Autònoma de Barcelona, Université Paris 13, and Aarhus Universitet for their hospitality. In particular, the origin of this project, and many of the results, go back to a three week period in the spring of 2001, when four of the authors met in Aberdeen.

\section{A QUiCK REVIEW OF $p$-LOCAL FINITE GROUPS}

We first recall the definitions of a fusion system and a saturated fusion system, in the form given in BLO2]. For any group $G$, and any pair of subgroups $H, K \leq G$, we set

$$
N_{G}(H, K)=\left\{x \in G \mid x H x^{-1} \leq K\right\}
$$

let $c_{x}$ denote conjugation by $x\left(c_{x}(g)=x g x^{-1}\right)$, and set

$$
\operatorname{Hom}_{G}(H, K)=\left\{c_{x} \in \operatorname{Hom}(H, K) \mid x \in N_{G}(H, K)\right\} \cong N_{G}(H, K) / C_{G}(H) .
$$

By analogy, we also write

$$
\operatorname{Aut}_{G}(H)=\operatorname{Hom}_{G}(H, H)=\left\{c_{x} \in \operatorname{Aut}(H) \mid x \in N_{G}(H)\right\} \cong N_{G}(H) / C_{G}(H) .
$$

Definition 1.1 ([Pu1] and [BLO2, Definition 1.1]). A fusion system over a finite $p$-group $S$ is a category $\mathcal{F}$, where $\operatorname{Ob}(\mathcal{F})$ is the set of all subgroups of $S$, and which satisfies the following two properties for all $P, Q \leq S$ :

- $\operatorname{Hom}_{S}(P, Q) \subseteq \operatorname{Hom}_{\mathcal{F}}(P, Q) \subseteq \operatorname{Inj}(P, Q)$; and

- each $\varphi \in \operatorname{Hom}_{\mathcal{F}}(P, Q)$ is the composite of an isomorphism in $\mathcal{F}$ followed by an inclusion.

The following additional definitions and conditions are needed in order for these systems to be very useful. If $\mathcal{F}$ is a fusion system over a finite $p$-subgroup $S$, then two subgroups $P, Q \leq S$ are said to be $\mathcal{F}$-conjugate if they are isomorphic as objects of the category $\mathcal{F}$.

Definition 1.2 ([י1]; see BLO2, Def. 1.2]). Let $\mathcal{F}$ be a fusion system over a $p$-group $S$.

- A subgroup $P \leq S$ is fully centralized in $\mathcal{F}$ if $\left|C_{S}(P)\right| \geq\left|C_{S}\left(P^{\prime}\right)\right|$ for all $P^{\prime} \leq S$ which is $\mathcal{F}$-conjugate to $P$. 
- A subgroup $P \leq S$ is fully normalized in $\mathcal{F}$ if $\left|N_{S}(P)\right| \geq\left|N_{S}\left(P^{\prime}\right)\right|$ for all $P^{\prime} \leq S$ which is $\mathcal{F}$-conjugate to $P$.

- $\mathcal{F}$ is a saturated fusion system if the following two conditions hold:

(I) For all $P \leq S$ which is fully normalized in $\mathcal{F}, P$ is fully centralized in $\mathcal{F}$ and $\operatorname{Aut}_{S}(P) \in \operatorname{Syl}_{p}\left(\operatorname{Aut}_{\mathcal{F}}(P)\right)$.

(II) If $P \leq S$ and $\varphi \in \operatorname{Hom}_{\mathcal{F}}(P, S)$ are such that $\varphi P$ is fully centralized, and if we set

$$
N_{\varphi}=\left\{g \in N_{S}(P) \mid \varphi c_{g} \varphi^{-1} \in \operatorname{Aut}_{S}(\varphi P)\right\},
$$

then there is $\bar{\varphi} \in \operatorname{Hom}_{\mathcal{F}}\left(N_{\varphi}, S\right)$ such that $\left.\bar{\varphi}\right|_{P}=\varphi$.

If $G$ is a finite group and $S \in \operatorname{Syl}_{p}(G)$, then by [BLO2, Proposition 1.3], the category $\mathcal{F}_{S}(G)$ defined by letting $\operatorname{Ob}\left(\mathcal{F}_{S}(G)\right)$ be the set of all subgroups of $S$ and setting $\operatorname{Mor}_{\mathcal{F}_{S}(G)}(P, Q)=\operatorname{Hom}_{G}(P, Q)$ is a saturated fusion system.

An alternative pair of axioms for a fusion system being saturated have been given by Radu Stancu [St]. He showed that axioms (I) and (II) above are equivalent to the two axioms:

$\left(\mathrm{I}^{\prime}\right) \operatorname{Inn}(S) \in \operatorname{Syl}_{p}\left(\operatorname{Aut}_{\mathcal{F}}(S)\right)$.

(II') If $P \leq S$ and $\varphi \in \operatorname{Hom}_{\mathcal{F}}(P, S)$ are such that $\varphi P$ is fully normalized, and if we set

$$
N_{\varphi}=\left\{g \in N_{S}(P) \mid \varphi c_{g} \varphi^{-1} \in \operatorname{Aut}_{S}(\varphi P)\right\}
$$

then there is $\bar{\varphi} \in \operatorname{Hom}_{\mathcal{F}}\left(N_{\varphi}, S\right)$ such that $\left.\bar{\varphi}\right|_{P}=\varphi$.

The following consequence of conditions (I) and (II) above will be needed several times throughout the paper.

Lemma 1.3. Let $\mathcal{F}$ be a saturated fusion system over a p-group $S$. Let $P, P^{\prime} \leq S$ be a pair of $\mathcal{F}$-conjugate subgroups such that $P^{\prime}$ is fully normalized in $\mathcal{F}$. Then there is a homomorphism $\alpha \in \operatorname{Hom}_{\mathcal{F}}\left(N_{S}(P), N_{S}\left(P^{\prime}\right)\right)$ such that $\alpha(P)=P^{\prime}$.

Proof. This is shown in BLO2, Proposition A.2(b)].

In this paper, it will sometimes be necessary to work with fusion systems which are not saturated. This is why we have emphasized the difference between fusion systems and saturated fusion systems, in the above definitions.

We next specify certain collections of subgroups relative to a given fusion system.

Definition 1.4. Let $\mathcal{F}$ be a fusion system over a finite $p$-subgroup $S$.

- A subgroup $P \leq S$ is $\mathcal{F}$-centric if $C_{S}\left(P^{\prime}\right)=Z\left(P^{\prime}\right)$ for all $P^{\prime} \leq S$ which is $\mathcal{F}$-conjugate to $P$.

- A subgroup $P \leq S$ is $\mathcal{F}$-radical if $\operatorname{Out}_{\mathcal{F}}(P)$ is $p$-reduced; i.e., if $O_{p}\left(\operatorname{Out}_{\mathcal{F}}(P)\right)$ $=1$.

- For any $P \leq S$ which is fully centralized in $\mathcal{F}$, the centralizer fusion system $C_{\mathcal{F}}(P)$ is the fusion system over $C_{S}(P)$ defined by setting

$\operatorname{Hom}_{C_{\mathcal{F}}(P)}\left(Q, Q^{\prime}\right)=\left\{\left.\alpha\right|_{Q}\left|\alpha \in \operatorname{Hom}_{\mathcal{F}}\left(Q P, Q^{\prime} P\right), \alpha\right|_{P}=\operatorname{Id}_{P}, \alpha(Q) \leq Q^{\prime}\right\}$.

A subgroup $P \leq S$ is $\mathcal{F}$-quasicentric if for all $P^{\prime} \leq S$ which is $\mathcal{F}$-conjugate to $P$ and fully centralized in $\mathcal{F}, C_{\mathcal{F}}\left(P^{\prime}\right)$ is the fusion system of the $p$-group $C_{S}\left(P^{\prime}\right)$.

- $\mathcal{F}^{c} \subseteq \mathcal{F}^{q} \subseteq \mathcal{F}$ denote the full subcategories of $\mathcal{F}$ whose objects are the $\mathcal{F}$-centric subgroups, and $\mathcal{F}$-quasicentric subgroups, respectively, of $S$. 
If $\mathcal{F}=\mathcal{F}_{S}(G)$ for some finite group $G$, then $P \leq S$ is $\mathcal{F}$-centric if and only if $P$ is $p$-centric in $G$ (i.e., $Z(P) \in \operatorname{Syl}_{p}\left(C_{G}(P)\right)$ ), and $P$ is $\mathcal{F}$-radical if and only if $N_{G}(P) /\left(P \cdot C_{G}(P)\right)$ is $p$-reduced. Also, $P$ is $\mathcal{F}$-quasicentric if and only if $C_{G}(P)$ contains a normal subgroup of order prime to $p$ and of $p$-power index.

In fact, when working with $p$-local finite groups, it suffices to have a fusion system $\mathcal{F}^{c}$ defined on the centric subgroups of $S$, and which satisfies axioms (I) and (II) above for those centric subgroups. In other words, fusion systems defined only on the centric subgroups are equivalent to fusion systems defined on all subgroups, as described in the following theorem.

Theorem 1.5. Fix a p-group $S$ and a fusion system $\mathcal{F}$ over $S$.

(a) Assume $\mathcal{F}$ is saturated. Then each morphism in $\mathcal{F}$ is a composite of restrictions of morphisms between subgroups of $S$ which are $\mathcal{F}$-centric, $\mathcal{F}$-radical, and fully normalized in $\mathcal{F}$. More precisely, for each $P, P^{\prime} \leq S$ and each $\varphi \in \operatorname{Iso}_{\mathcal{F}}\left(P, P^{\prime}\right)$, there are subgroups $P=P_{0}, P_{1}, \ldots, P_{k}=P^{\prime}$, subgroups $Q_{i} \geq\left\langle P_{i-1}, P_{i}\right\rangle(i=1, \ldots, k)$ which are $\mathcal{F}$-centric, $\mathcal{F}$-radical, and fully normalized in $\mathcal{F}$, and automorphisms $\varphi_{i} \in \operatorname{Aut}_{\mathcal{F}}\left(Q_{i}\right)$, such that $\varphi_{i}\left(P_{i-1}\right)=P_{i}$ for all $i$ and $\varphi=\left.\varphi_{k} \circ \cdots \circ \varphi_{1}\right|_{P}$.

(b) Assume conditions (I) and (II) in Definition 1.2 are satisfied for all $\mathcal{F}$ centric subgroups $P \leq S$. Assume also that each morphism in $\mathcal{F}$ is a composite of restrictions of morphisms between $\mathcal{F}$-centric subgroups of $S$. Then $\mathcal{F}$ is saturated.

Proof. Part (a) is Alperin's fusion theorem for saturated fusion systems, in the form shown in [BLO2, Theorem A.10]. Part (b) is a special case of [5A1, Theorem 2.2]: the case where $\mathcal{H}$ is the set of all $\mathcal{F}$-centric subgroups of $S$.

Theorem 1.5(a) will be used repeatedly throughout this paper. The following lemma is a first easy application of the theorem, and provides a very useful criterion for a subgroup to be quasicentric or not.

Lemma 1.6. Let $\mathcal{F}$ be a saturated fusion system over a p-group $S$. Then the following hold for any $P \leq S$ :

(a) Assume that $P \leq Q \leq P \cdot C_{S}(P)$ and $\operatorname{Id} \neq \alpha \in \operatorname{Aut}_{\mathcal{F}}(Q)$ are such that $\left.\alpha\right|_{P}=\operatorname{Id}_{P}$ and $\alpha$ has order prime to $p$. Then $P$ is not $\mathcal{F}$-quasicentric.

(b) Assume that $P$ is fully centralized in $\mathcal{F}$, and is not $\mathcal{F}$-quasicentric. Then there are $P \leq Q \leq P \cdot C_{S}(P)$ and $\operatorname{Id} \neq \alpha \in \operatorname{Aut}_{\mathcal{F}}(Q)$ such that $Q$ is $\mathcal{F}$ centric, $\left.\alpha\right|_{P}=\operatorname{Id}_{P}$, and $\alpha$ has order prime to $p$.

Proof. (a) Fix any $P^{\prime}$ which is $\mathcal{F}$-conjugate to $P$ and fully centralized in $\mathcal{F}$. By axiom (II), there is $\varphi \in \operatorname{Hom}_{\mathcal{F}}(Q, S)$ such that $\varphi(P)=P^{\prime}$; set $Q^{\prime}=\varphi(Q)$. Thus $\left.\varphi \alpha \varphi^{-1}\right|_{C_{Q^{\prime}}\left(P^{\prime}\right)}$ is an automorphism in $C_{\mathcal{F}}\left(P^{\prime}\right)$ whose order is not a power of $p$, so $C_{\mathcal{F}}\left(P^{\prime}\right)$ is not the fusion system of $C_{S}\left(P^{\prime}\right)$, and $P$ is not $\mathcal{F}$-quasicentric.

(b) Assume that $P$ is fully centralized in $\mathcal{F}$ and not $\mathcal{F}$-quasicentric. Then $C_{\mathcal{F}}(P)$ strictly contains the fusion system of $C_{S}(P)$ (since $C_{\mathcal{F}}\left(P^{\prime}\right)$ is isomorphic as a category to $C_{\mathcal{F}}(P)$ for all $P^{\prime}$ which is $\mathcal{F}$-conjugate to $P$ and fully centralized in $\mathcal{F})$. Since $C_{\mathcal{F}}(P)$ is saturated by [BLO2, Proposition A.6], Theorem 1.5(a) implies there is a subgroup $Q \leq C_{S}(P)$ which is $C_{\mathcal{F}}(P)$-centric and fully normalized in $C_{\mathcal{F}}(P)$, and such that $\operatorname{Aut}_{C_{\mathcal{F}}(P)}(Q) \supsetneqq \operatorname{Aut}_{C_{S}(P)}(Q)$. Since $Q$ is fully normalized, $\operatorname{Aut}_{C_{S}(P)}(Q)$ is a Sylow $p$-subgroup of $\operatorname{Aut}_{C_{\mathcal{F}}(P)}(Q)$, and hence this last group is 
not a $p$-group. Also, by [BLO2, Proposition 2.5(a)], $P Q$ is $\mathcal{F}$-centric since $Q$ is $C_{\mathcal{F}}(P)$-centric.

Since orbit categories — both of fusion systems and of groups — will play a role in certain proofs in the last three sections, we define them here.

Definition 1.7. $\quad$ (a) If $\mathcal{F}$ is a fusion system over a $p$-group $S$, then $\mathcal{O}^{c}(\mathcal{F})$ (the centric orbit category of $\mathcal{F}$ ) is the category whose objects are the $\mathcal{F}$-centric subgroups of $S$, and whose morphism sets are given by

$$
\operatorname{Mor}_{\mathcal{O}^{c}(\mathcal{F})}(P, Q)=\operatorname{Rep}_{\mathcal{F}}(P, Q) \stackrel{\text { def }}{=} Q \backslash \operatorname{Hom}_{\mathcal{F}}(P, Q) .
$$

Let $\mathcal{Z}_{\mathcal{F}}: \mathcal{O}^{c}(\mathcal{F}) \rightarrow \mathbb{Z}_{(p)}$-mod be the functor which sends $P$ to $Z(P)$ and $[\varphi]$ (the class of $\varphi \in \operatorname{Hom}_{\mathcal{F}}(P, Q)$ ) to $Z(Q) \stackrel{\varphi^{-1}}{\longrightarrow} Z(P)$.

(b) If $G$ is a finite group and $S \in \operatorname{Syl}_{p}(G)$, then $\mathcal{O}_{S}^{c}(G)$ (the centric orbit category of $G$ ) is the category whose objects are the subgroups of $S$ which are $p$-centric in $G$, and where

$$
\operatorname{Mor}_{\mathcal{O}_{S}^{c}(G)}(P, Q)=Q \backslash N_{G}(P, Q) \cong \operatorname{Map}_{G}(G / P, G / Q) \text {. }
$$

Let $\mathcal{Z}_{G}: \mathcal{O}_{S}^{c}(G) \rightarrow \mathbb{Z}_{(p)}$-mod be the functor which sends $P$ to $Z(P)$ and $[g]$ (the class of $g \in N_{G}(P, Q)$ ) to conjugation by $g^{-1}$.

We now turn to linking systems associated to abstract fusion systems.

Definition 1.8 ([BLO2, Def. 1.7]). Let $\mathcal{F}$ be a fusion system over the $p$-group $S$. A centric linking system associated to $\mathcal{F}$ is a category $\mathcal{L}$ whose objects are the $\mathcal{F}$ centric subgroups of $S$, together with a functor $\pi: \mathcal{L} \longrightarrow \mathcal{F}^{c}$, and "distinguished" monomorphisms $P \stackrel{\delta_{P}}{\longrightarrow} \operatorname{Aut}_{\mathcal{L}}(P)$ for each $\mathcal{F}$-centric subgroup $P \leq S$, which satisfy the following conditions:

(A) $\pi$ is the identity on objects. For each pair of objects $P, Q \in \mathcal{L}, Z(P)$ acts freely on $\operatorname{Mor}_{\mathcal{L}}(P, Q)$ by composition (upon identifying $Z(P)$ with $\left.\delta_{P}(Z(P)) \leq \operatorname{Aut}_{\mathcal{L}}(P)\right)$, and $\pi$ induces a bijection

$$
\operatorname{Mor}_{\mathcal{L}}(P, Q) / Z(P) \stackrel{\cong}{\longrightarrow} \operatorname{Hom}_{\mathcal{F}}(P, Q) \text {. }
$$

(B) For each $\mathcal{F}$-centric subgroup $P \leq S$ and each $x \in P, \pi\left(\delta_{P}(x)\right)=c_{x} \in$ $\operatorname{Aut}_{\mathcal{F}}(P)$.

(C) For each $f \in \operatorname{Mor}_{\mathcal{L}}(P, Q)$ and each $x \in P$, the following square commutes in $\mathcal{L}$ :

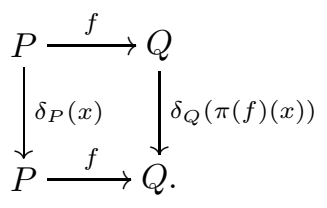

A $p$-local finite group is defined to be a triple $(S, \mathcal{F}, \mathcal{L})$, where $S$ is a finite $p$ group, $\mathcal{F}$ is a saturated fusion system over $S$, and $\mathcal{L}$ is a centric linking system associated to $\mathcal{F}$. The classifying space of the triple $(S, \mathcal{F}, \mathcal{L})$ is the $p$-completed nerve $|\mathcal{L}|_{p}^{\wedge}$.

For any finite group $G$ with Sylow $p$-subgroup $S$, a category $\mathcal{L}_{S}^{c}(G)$ was defined in BLO1, whose objects are the $p$-centric subgroups of $G$, and whose morphism sets are defined by

$$
\operatorname{Mor}_{\mathcal{L}_{S}^{c}(G)}(P, Q)=N_{G}(P, Q) / O^{p}\left(C_{G}(P)\right) .
$$


Since $C_{G}(P)=Z(P) \times O^{p}\left(C_{G}(P)\right)$ when $P$ is $p$-centric in $G, \mathcal{L}_{S}^{c}(G)$ is easily seen to satisfy conditions (A), (B), and (C) above, and hence is a centric linking system associated to $\mathcal{F}_{S}(G)$. Thus $\left(S, \mathcal{F}_{S}(G), \mathcal{L}_{S}^{c}(G)\right)$ is a $p$-local finite group, with classifying space $\left|\mathcal{L}_{S}^{c}(G)\right|_{p}^{\wedge} \simeq B G_{p}^{\wedge}$ (see [BLO1, Proposition 1.1]).

It will be of crucial importance in this paper that any centric linking system associated to $\mathcal{F}$ can be extended to a quasicentric linking system; a linking system with similar properties, whose objects are the $\mathcal{F}$-quasicentric subgroups of $S$. We first make more precise what this means.

Definition 1.9. Let $\mathcal{F}$ be any saturated fusion system over a $p$-group $S$. A quasicentric linking system associated to $\mathcal{F}$ consists of a category $\mathcal{L}^{q}$ whose objects are the $\mathcal{F}$-quasicentric subgroups of $S$, together with a functor $\pi: \mathcal{L}^{q} \longrightarrow \mathcal{F}^{q}$, and distinguished monomorphisms

$$
P \cdot C_{S}(P) \stackrel{\delta_{P}}{\longrightarrow} \operatorname{Aut}_{\mathcal{L}^{q}}(P),
$$

which satisfy the following conditions:

$(\mathrm{A})_{q} \pi$ is the identity on objects and surjective on morphisms. For each pair of objects $P, Q \in \mathcal{L}^{q}$ such that $P$ is fully centralized, $C_{S}(P)$ acts freely on $\operatorname{Mor}_{\mathcal{L}^{q}}(P, Q)$ by composition (upon identifying $C_{S}(P)$ with the subgroup $\left.\delta_{P}\left(C_{S}(P)\right) \leq \operatorname{Aut}_{\mathcal{L}^{q}}(P)\right)$, and $\pi$ induces a bijection

$$
\operatorname{Mor}_{\mathcal{L}^{q}}(P, Q) / C_{S}(P) \stackrel{\cong}{\longrightarrow} \operatorname{Hom}_{\mathcal{F}}(P, Q) \text {. }
$$

$(\mathrm{B})_{q}$ For each $\mathcal{F}$-quasicentric subgroup $P \leq S$ and each $g \in P, \pi$ sends $\delta_{P}(g) \in$ $\operatorname{Aut}_{\mathcal{L}^{q}}(P)$ to $c_{g} \in \operatorname{Aut}_{\mathcal{F}}(P)$.

$(\mathrm{C})_{q}$ For each $f \in \operatorname{Mor}_{\mathcal{L}^{q}}(P, Q)$ and each $x \in P, f \circ \delta_{P}(x)=\delta_{Q}(\pi(f)(x)) \circ f$ in $\operatorname{Mor}_{\mathcal{L}^{q}}(P, Q)$.

$(\mathrm{D})_{q}$ For each $\mathcal{F}$-quasicentric subgroup $P \leq S$, there is some $\iota_{P} \in \operatorname{Mor}_{\mathcal{L}^{q}}(P, S)$ such that $\pi\left(\iota_{P}\right)=\operatorname{incl}_{P}^{S} \in \operatorname{Hom}(P, S)$, and such that for each $g \in P \cdot C_{S}(P)$, $\delta_{S}(g) \circ \iota_{P}=\iota_{P} \circ \delta_{P}(g)$ in $\operatorname{Mor}_{\mathcal{L}^{q}}(P, S)$.

If $P$ and $P^{\prime}$ are $\mathcal{F}$-conjugate and $\mathcal{F}$-quasicentric, then for any subgroup $Q \leq S$, $\operatorname{Mor}_{\mathcal{L}^{q}}(P, Q) \cong \operatorname{Mor}_{\mathcal{L}^{q}}\left(P^{\prime}, Q\right)$ and $\operatorname{Hom}_{\mathcal{F}}(P, Q) \cong \operatorname{Hom}_{\mathcal{F}}\left(P^{\prime}, Q\right)$, while the centralizers $C_{S}(P)$ and $C_{S}\left(P^{\prime}\right)$ need not have the same order. This is why condition $(\mathrm{A})_{q}$ makes sense only if we assume that $P$ is fully centralized; i.e., that $C_{S}(P)$ is as large as possible. When $P$ is $\mathcal{F}$-centric, then this condition is irrelevant, since every subgroup $P^{\prime}$ which is $\mathcal{F}$-conjugate to $P$ is fully centralized $\left(C_{S}\left(P^{\prime}\right)=Z\left(P^{\prime}\right) \cong Z(P)\right)$.

Note that $(\mathrm{D})_{q}$ is a special case of $(\mathrm{C})_{q}$ when $P$ is $\mathcal{F}$-centric; this is why the axiom is not needed for centric linking systems. We also note the following relation between these axioms:

Lemma 1.10. In the situation of Definition 1.9, axiom $(C)_{q}$ implies axiom $(B)_{q}$.

Proof. Fix an $\mathcal{F}$-quasicentric subgroup $P \leq S$, and an element $g \in P$. We apply $(\mathrm{C})_{q}$ with $f=\delta_{P}(g)$. For each $x \in P$, if we set $y=\pi\left(\delta_{P}(g)\right)(x)$, then $\delta_{P}(g) \circ \delta_{P}(x)=$ $\delta_{P}(y) \circ \delta_{P}(g)$. Since $\delta_{P}$ is an injective homomorphism, this implies that $g x=y g$, and thus that $y=c_{g}(x)$. So $\pi\left(\delta_{P}(g)\right)=c_{g}$.

When $(S, \mathcal{F}, \mathcal{L})$ is a $p$-local finite group, and $\mathcal{L}^{q}$ is a quasicentric linking system associated to $\mathcal{F}$, then we say that $\mathcal{L}^{q}$ extends $\mathcal{L}$ if the full subcategory of $\mathcal{L}^{q}$ with objects the $\mathcal{F}$-centric subgroups of $S$ is isomorphic to $\mathcal{L}$ via a functor which 
commutes with the projection functors to $\mathcal{F}$ and with the distinguished monomorphisms. In [5A1, Propositions $3.4 \& 3.12$ ], we constructed an explicit quasicentric linking system $\mathcal{L}^{q}$ associated to $\mathcal{F}$ and extending $\mathcal{L}$, and showed that it is unique up to an isomorphism of categories which preserves all of these structures. So from now on, we will simply refer to $\mathcal{L}^{q}$ as the quasicentric linking system associated to $(S, \mathcal{F}, \mathcal{L})$.

Condition $(\mathrm{D})_{q}$ above helps to motivate the following definition of inclusion morphisms in a quasicentric linking system.

Definition 1.11. Fix a $p$-local finite group $(S, \mathcal{F}, \mathcal{L})$, with associated quasicentric linking system $\mathcal{L}^{q}$.

(a) A morphism $\iota_{P} \in \operatorname{Mor}_{\mathcal{L}^{q}}(P, S)$ is an inclusion morphim if it satisfies the hypotheses of axiom (D) $)_{q}$ : if $\pi\left(\iota_{P}\right)=\operatorname{incl}_{P}^{S}$, and if $\delta_{S}(g) \circ \iota_{P}=\iota_{P} \circ \delta_{P}(g)$ in $\operatorname{Mor}_{\mathcal{L}^{q}}(P, S)$ for all $g \in P \cdot C_{S}(P)$. If $P=S$, then we also require that $\iota_{S}=\operatorname{Id}_{S}$.

(b) A compatible set of inclusions for $\mathcal{L}^{q}$ is a choice of morphisms $\left\{\iota_{P}^{Q}\right\}$ for all pairs of $\mathcal{F}$-quasicentric subgroups $P \leq Q$, such that $\iota_{P}^{Q} \in \operatorname{Mor}_{\mathcal{L}^{q}}(P, Q)$, such that $\iota_{P}^{R}=\iota_{Q}^{R} \circ \iota_{P}^{Q}$ for all $P \leq Q \leq R$, and such that $\iota_{P}^{S}$ is an inclusion morphism for each $P$.

The following properties of quasicentric linking systems were also proven in [5A1].

Proposition 1.12. The following hold for any $p$-local finite group $(S, \mathcal{F}, \mathcal{L})$, with associated quasicentric linking system $\mathcal{L}^{q}$ :

(a) The inclusion $\mathcal{L} \subseteq \mathcal{L}^{q}$ induces a homotopy equivalence $|\mathcal{L}| \simeq\left|\mathcal{L}^{q}\right|$ between geometric realizations. More generally, for any full subcategory $\mathcal{L}^{\prime} \subseteq \mathcal{L}^{q}$ which contains as objects all subgroups of $S$ which are $\mathcal{F}$-centric and $\mathcal{F}$ radical, the inclusion $\mathcal{L}^{\prime} \subseteq \mathcal{L}^{q}$ induces a homotopy equivalence $\left|\mathcal{L}^{\prime}\right| \simeq\left|\mathcal{L}^{q}\right|$.

(b) Let $\varphi \in \operatorname{Mor}_{\mathcal{L}^{q}}(P, R)$ and $\psi \in \operatorname{Mor}_{\mathcal{L}^{q}}(Q, R)$ be any pair of morphisms in $\mathcal{L}^{q}$ with the same target group such that $\operatorname{Im}(\pi(\varphi)) \leq \operatorname{Im}(\pi(\psi))$. Then there is a unique morphism $\chi \in \operatorname{Mor}_{\mathcal{L}^{q}}(P, Q)$ such that $\varphi=\psi \circ \chi$.

Proof. The homotopy equivalences $\left|\mathcal{L}^{q}\right| \simeq|\mathcal{L}| \simeq\left|\mathcal{L}^{\prime}\right|$ are shown in 5A1, Theorem 3.5]. Point (b) is shown in [5A1, Lemma 3.6].

Point (b) above will be frequently used throughout the paper. In particular, it makes it possible to embed the linking system of $S$ (or an appropriate full subcategory) in $\mathcal{L}^{q}$, depending on the choice of an inclusion morphisms $\iota_{P}$ as defined above, for each object $P$. Such inclusion morphisms always exist by axiom $(\mathrm{D})_{q}$. In the following proposition, $\left.\mathcal{L}_{S}(S)\right|_{\mathrm{Ob}\left(\mathcal{L}^{q}\right)}$ denotes the full subcategory of $\mathcal{L}_{S}(S)$ whose objects are the $\mathcal{F}$-quasicentric subgroups of $S$.

In general, for a functor $F: \mathcal{C} \rightarrow \mathcal{C}^{\prime}$, and objects $c, d \in \operatorname{Ob}(\mathcal{C})$, we let $F_{c, d}$ denote the map from $\operatorname{Mor}_{\mathcal{C}}(c, d)$ to $\operatorname{Mor}_{\mathcal{C}^{\prime}}(F(c), F(d))$ induced by $F$.

Proposition 1.13. Fix a p-local finite group $(S, \mathcal{F}, \mathcal{L})$. Let $\mathcal{L}^{q}$ be its associated quasicentric linking system, and let $\pi: \mathcal{L}^{q} \longrightarrow \mathcal{F}^{q}$ be the projection. Then any choice of inclusion morphisms $\iota_{P}=\iota_{P}^{S} \in \operatorname{Mor}_{\mathcal{L}^{q}}(P, S)$, for all $\mathcal{F}$-quasicentric subgroups $P \leq S$, extends to a unique inclusion of categories

$$
\delta:\left.\mathcal{L}_{S}(S)\right|_{\mathrm{Ob}\left(\mathcal{L}^{q}\right)} \longrightarrow \mathcal{L}^{q}
$$


such that $\delta_{P, S}(1)=\iota_{P}$ for all $P$; and such that

(a) $\delta_{P, P}(g)=\delta_{P}(g)$ for all $g \in P \cdot C_{S}(P)$, and

(b) $\pi\left(\delta_{P, Q}(g)\right)=c_{g} \in \operatorname{Hom}(P, Q)$ for all $g \in N_{S}(P, Q)$.

In addition, the following hold:

(c) If we set $\iota_{P}^{Q}=\delta_{P, Q}(1)$ for all $P \leq Q$, then $\left\{\iota_{P}^{Q}\right\}$ is a compatible set of inclusions for $\mathcal{L}^{q}$.

(d) For any $P \triangleleft Q \leq S$, where $P$ and $Q$ are both $\mathcal{F}$-quasicentric and $P$ is fully centralized in $\mathcal{F}$, and any morphism $\psi \in \operatorname{Aut}_{\mathcal{L}^{q}}(P)$ which normalizes $\delta_{P, P}(Q)$, there is a unique $\widehat{\psi} \in \operatorname{Aut}_{\mathcal{L}^{q}}(Q)$ such that $\widehat{\psi} \circ \iota_{P}^{Q}=\iota_{P}^{Q} \circ \psi$. Furthermore, for any $g \in Q, \psi \delta_{P, P}(g) \psi^{-1}=\delta_{P, P}(\pi(\widehat{\psi})(g))$.

(e) Every morphism $\varphi \in \operatorname{Mor}_{\mathcal{L}^{q}}(P, Q)$ in $\mathcal{L}^{q}$ is a composite $\varphi=\iota_{P^{\prime}}^{Q} \circ \varphi^{\prime}$ for a unique morphism $\varphi^{\prime} \in \operatorname{Iso}_{\mathcal{L}^{q}}\left(P, P^{\prime}\right)$, where $P^{\prime}=\operatorname{Im}(\pi(\varphi))$.

Proof. For each $P$ and $Q$, and each $g \in N_{S}(P, Q)$, there is by Proposition 1.12(b) a unique morphism $\delta_{P, Q}(g)$ such that

$$
\delta_{S}(g) \circ \iota_{P}=\iota_{Q} \circ \delta_{P, Q}(g) .
$$

This defines $\delta$ on morphism sets, and also allows us to define $\iota_{P}^{Q}=\delta_{P, Q}(1)$. Then by the axioms in Definition 1.9, $\left\{\iota_{P}^{Q}\right\}$ is a compatible set of inclusions for $\mathcal{L}^{q}$, and $\delta$ is a functor which satisfies (a), (b), and (c). Point (e) is a special case of Proposition 1.12(b) (where $P^{\prime}=\operatorname{Im}(\pi(\varphi))$ ).

If $\delta_{P, Q}(g)=\delta_{P, Q}\left(g^{\prime}\right)$ for $g, g^{\prime} \in N_{G}(P, Q)$, then $\delta_{S}(g) \circ \iota_{P}=\delta_{S}\left(g^{\prime}\right) \circ \iota_{P}$, and hence $g=g^{\prime}$ by [5A1, Lemma 3.9]. Thus each $\delta_{P, Q}$ is injective.

It remains to prove (d). Set $\varphi=\pi(\psi) \in \operatorname{Aut}_{\mathcal{F}}(P)$ for short. Since $\psi$ normalizes $\delta_{P, P}(Q)$, for all $g \in Q$ there is $h \in Q$ such that $\psi \delta_{P, P}(g) \psi^{-1}=\delta_{P, P}(h)$, and this implies the relation $\varphi c_{g} \varphi^{-1}=c_{h}$ in $\operatorname{Aut}_{\mathcal{F}}(P)$. Thus $Q$ is contained in $N_{\varphi}$. So by axiom (II) (and since $P$ is fully centralized), $\varphi$ extends to $\bar{\varphi} \in \operatorname{Iso}_{\mathcal{F}}\left(Q, Q^{\prime}\right)$ for some $P \triangleleft Q^{\prime} \leq S$. Let $\widehat{\psi}_{0} \in \operatorname{Iso}_{\mathcal{L}^{q}}\left(Q, Q^{\prime}\right)$ be any lifting of $\bar{\varphi}$ to $\mathcal{L}^{q}$.

By axiom $(\mathrm{A})_{q}$ (and since $\psi$ is an isomorphism), there is $x \in C_{S}(P)$ such that $\iota_{P}^{Q^{\prime}} \circ \delta_{P}(x) \circ \psi=\widehat{\psi}_{0} \circ \iota_{P}^{Q}$. By $(\mathrm{C})_{q}, \widehat{\psi}_{0} \delta_{Q}(Q) \widehat{\psi}_{0}^{-1}=\delta_{Q^{\prime}}\left(Q^{\prime}\right)$, and hence after restriction, $\delta_{P}(x) \circ \psi$ conjugates $\delta_{P, P}(Q)$ to $\delta_{P, P}\left(Q^{\prime}\right)$. Since $\psi$ normalizes $\delta_{P, P}(Q)$, this shows that $\delta_{P}(x)$ conjugates $\delta_{P, P}(Q)$ to $\delta_{P, P}\left(Q^{\prime}\right)$, and hence (since $\delta_{P, P}$ is injective) that $x Q x^{-1}=Q^{\prime}$. We thus have the following commutative diagram:

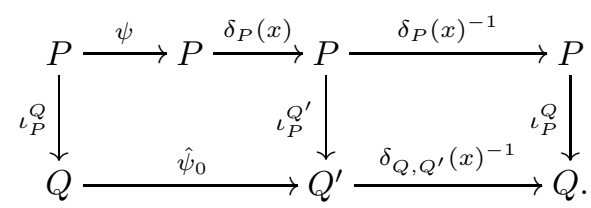

So if we set $\widehat{\psi}=\delta_{Q, Q^{\prime}}(x)^{-1} \circ \widehat{\psi}_{0}$, then $\widehat{\psi} \in \operatorname{Aut}_{\mathcal{L}^{q}}(Q)$ and $\iota_{P}^{Q} \circ \psi=\widehat{\psi} \circ \iota_{P}^{Q}$.

The uniqueness of $\widehat{\psi}$ follows from [5A1, Lemma 3.9]. Finally, for any $g \in Q$, $\widehat{\psi} \delta_{Q}(g) \widehat{\psi}^{-1}=\delta_{Q}(\pi(\widehat{\psi})(g))$ by $(\mathrm{C})_{q}$, and hence $\psi \delta_{P, P}(g) \psi^{-1}=\delta_{P, P}(\pi(\widehat{\psi})(g))$ since morphisms have unique restrictions (Proposition 1.12(b) again).

Once we have fixed a compatible set of inclusions $\left\{\iota_{P}^{Q}\right\}$ in a linking system $\mathcal{L}^{q}$, then for any $\varphi \in \operatorname{Mor}_{\mathcal{L}^{q}}(P, Q)$, and any $P^{\prime} \leq P$ and $Q^{\prime} \leq Q$ such that $\pi(\varphi)\left(P^{\prime}\right) \leq$ $Q^{\prime}$, there is a unique morphism $\bar{\varphi} \in \operatorname{Mor}_{\mathcal{L}^{q}}\left(P^{\prime}, Q^{\prime}\right)$ such that $\iota_{Q^{\prime}}^{Q} \circ \bar{\varphi}=\varphi \circ \iota_{P^{\prime}}^{P}$. We think of $\bar{\varphi}$ as the restriction of $\varphi$. 
Note, however, that all of this depends on the choice of inclusion morphisms $\iota_{P} \in \operatorname{Mor}_{\mathcal{L}^{q}}(P, S)$ which satisfy the hypotheses of axiom $(\mathrm{D})_{q}$, and that not just any lifting of the inclusion incl $\in \operatorname{Hom}_{\mathcal{F}}(P, S)$ can be chosen. To see why, assume for simplicity that $P$ is also fully centralized. From the axioms in Definition 1.9 and Proposition 1.12(b), we see that if $\iota_{P}, \iota_{P}^{\prime} \in \operatorname{Mor}_{\mathcal{L}^{q}}(P, S)$ are two liftings of incl $\in \operatorname{Hom}_{\mathcal{F}}(P, S)$, then $\iota_{P}^{\prime}=\iota_{P} \circ \delta_{P}(g)$ for some unique $g \in C_{S}(P)$. But if $\iota_{P}$ satisfies the conditions of $(\mathrm{D})_{q}$, then $\iota_{P}^{\prime}$ also satisfies those conditions only if $g \in Z\left(C_{S}(P)\right)$.

One situation where the choice of inclusion morphisms is useful is when describing the fundamental group of $|\mathcal{L}|$ or of its $p$-completion. For any group $\Gamma$, we let $\mathcal{B}(\Gamma)$ denote the category with one object, and with morphism monoid the group $\Gamma$. Recall that $|\mathcal{L}| \simeq\left|\mathcal{L}^{q}\right|$ (Proposition 1.12(a)), so we can work with either of these categories; we will mostly state the results for $\left|\mathcal{L}^{q}\right|$. Let the vertex $S$ be the basepoint of $\left|\mathcal{L}^{q}\right|$. For each morphism $\varphi \in \operatorname{Mor}_{\mathcal{L}^{q}}(P, Q)$, let $J(\varphi) \in \pi_{1}\left(\left|\mathcal{L}^{q}\right|\right)$ denote the homotopy class of the loop $\iota_{Q} \cdot \varphi \cdot \iota_{P}{ }^{-1}$ in $\left|\mathcal{L}^{q}\right|$ (where paths are composed from right to left). This defines a functor

$$
J: \mathcal{L}^{q} \longrightarrow \mathcal{B}\left(\pi_{1}\left(\left|\mathcal{L}^{q}\right|\right)\right),
$$

where all objects are sent to the unique object of $\mathcal{B}\left(\pi_{1}\left(\left|\mathcal{L}^{q}\right|\right)\right)$, and where all inclusion morphisms are sent to the identity. Let $j: S \rightarrow \pi_{1}\left(\left|\mathcal{L}^{q}\right|\right)$ denote the composite of $J$ with the distinguished monomorphism $\delta_{S}: S \rightarrow \operatorname{Aut}_{\mathcal{L}}(S)$.

The next proposition describes how $J$ is universal among functors of this type, and also includes some other technical results for later use about the structure of $\pi_{1}(|\mathcal{L}|)$.

Proposition 1.14. Let $(S, \mathcal{F}, \mathcal{L})$ be a p-local finite group, and let $\mathcal{L}^{q}$ be the associated quasicentric linking system. Assume a compatible set of inclusions $\left\{\iota_{P}^{Q}\right\}$ has been chosen for $\mathcal{L}^{q}$. Then the following hold:

(a) For any group $\Gamma$, and any functor $\lambda: \mathcal{L}^{q} \longrightarrow \mathcal{B}(\Gamma)$ which sends inclusions to the identity, there is a unique homomorphism $\bar{\lambda}: \pi_{1}\left(\left|\mathcal{L}^{q}\right|\right) \longrightarrow \Gamma$ such that $\lambda=\mathcal{B}(\bar{\lambda}) \circ J$.

(b) For $g \in P \leq S$ with $P \mathcal{F}$-quasicentric, $J\left(\delta_{P}(g)\right)=J\left(\delta_{S}(g)\right)$. In particular, $J\left(\delta_{P}(g)\right)=1$ in $\pi_{1}\left(\left|\mathcal{L}^{q}\right|\right)$ if and only if $\delta_{P}(g)$ is nulhomotopic as a loop based at the vertex $P$ of $\left|\mathcal{L}^{q}\right|$.

(c) If $\alpha \in \operatorname{Mor}_{\mathcal{L}^{q}}(P, Q)$, and $\pi(\alpha)(x)=y$, then $j(y)=J(\alpha) j(x) J(\alpha)^{-1}$ in $\pi_{1}\left(\left|\mathcal{L}^{q}\right|\right)$.

(d) If $x$ and $y$ are $\mathcal{F}$-conjugate elements of $S$, then $j(x)$ and $j(y)$ are conjugate in $\pi_{1}\left(\left|\mathcal{L}^{q}\right|\right)$.

Proof. Clearly, any functor $\lambda: \mathcal{L}^{q} \rightarrow \mathcal{B}(\Gamma)$ induces a homomorphism $\bar{\lambda}=\pi_{1}(|\lambda|)$ between the fundamental groups of their geometric realizations. If $\lambda$ sends inclusion morphisms to the identity, then $\lambda=\mathcal{B}(\bar{\lambda}) \circ J$ by definition of $J$.

The other points follow easily, using condition $(\mathrm{C})_{q}$ for quasicentric linking systems. Point (d) is shown by first reducing to a map between centric subgroups of $S$ which sends $x$ to $y$.

We finish this introductory section with two unrelated results which will be needed later in the paper. The first is a standard, group theoretic lemma. 
Lemma 1.15. Let $Q \triangleleft P$ be p-groups. If $\alpha$ is a $p^{\prime}$-automorphism of $P$ which acts as the identity on $Q$ and on $P / Q$, then $\alpha=\operatorname{Id}_{P}$. Equivalently, the group of all automorphisms of $P$ which restrict to the identity on $Q$ and on $P / Q$ is a p-group.

Proof. See [Go, Corollary 5.3.3].

The following proposition will only be used in Section 4, but we include it here because it seems to be of wider interest. Note, for any fusion system $\mathcal{F}$ over $S$, any subgroup $P \leq S$ fully normalized in $\mathcal{F}$, and any $P^{\prime}$ which is $S$-conjugate to $P$, that $P^{\prime}$ is also fully normalized in $\mathcal{F}$ since $N_{S}\left(P^{\prime}\right)$ is $S$-conjugate to $N_{S}(P)$.

Proposition 1.16. Let $\mathcal{F}$ be a saturated fusion system over a p-group $S$. Then for any subgroup $P \leq S$, the set of $S$-conjugacy classes of subgroups $\mathcal{F}$-conjugate to $P$ and fully normalized in $\mathcal{F}$ has order prime to $p$.

Proof. By [BLO2, Proposition 5.5], there is an $(S, S)$-biset $\Omega$ which, when regarded as a set with $(S \times S)$-action, satisfies the following three conditions:

(a) The isotropy subgroup of each point in $\Omega$ is of the form

$$
P_{\varphi} \stackrel{\text { def }}{=}\{(x, \varphi(x)) \mid x \in P\}
$$

for some $P \leq S$ and some $\varphi \in \operatorname{Hom}_{\mathcal{F}}(P, S)$.

(b) For each $P \leq S$ and each $\varphi \in \operatorname{Hom}_{\mathcal{F}}(P, S)$, the two structures of $(S \times P)$-set on $\Omega$ obtained by restriction and by $\operatorname{Id} \times \varphi$ are isomorphic.

(c) $|\Omega| /|S| \equiv 1(\bmod p)$.

Note that by (a), the actions of $S \times 1$ and $1 \times S$ on $\Omega$ are both free.

Now fix a subgroup $P \leq S$. Set $S_{2}=1 \times S$ for short, and let $\Omega_{0} \subseteq \Omega$ be the subset such that $\Omega_{0} / S_{2}=\left(\Omega / S_{2}\right)^{P}$. In other words, $\Omega_{0}$ is the set of all $x \in \Omega$ such that for each $g \in P$, there is some $h \in S$ satisfying $(g, h) \cdot x=x$. Since the action of $S_{2}$ on $\Omega$ is free, this element $h \in S$ is uniquely determined for each $x \in \Omega_{0}$ and $g \in P$. Let $\theta(x): P \rightarrow S$ denote the function such that for each $g \in P$, $(g, \theta(x)(g)) \cdot x=x$. The isotropy subgroup at $x$ of the $(P \times S)$-action is thus the subgroup $P_{\theta(x)}=\{(g, \theta(x)(g)) \mid g \in P\}$; and by $(\mathrm{a}), \theta(x) \in \operatorname{Hom}_{\mathcal{F}}(P, S)$. This defines a map

$$
\theta: \Omega_{0} \rightarrow \operatorname{Hom}_{\mathcal{F}}(P, S)
$$

By definition, for each $\varphi \in \operatorname{Hom}_{\mathcal{F}}(P, S), \theta^{-1}(\varphi)$ is the set of elements of $\Omega$ fixed by $P_{\varphi}$. By condition (b) above, the action of $P \times P$ on $\Omega$ induced by the homomorphism $1 \times \varphi \in \operatorname{Hom}(P \times P, S \times S)$ is isomorphic to the action defined by restriction, and thus $\left|\theta^{-1}(\varphi)\right|=\mid \theta^{-1}$ (incl) $\mid$. This shows that the point inverses of $\theta$ all have the same fixed order $k$.

Now let $\operatorname{Rep}_{\mathcal{F}}(P, S)=\operatorname{Hom}_{\mathcal{F}}(P, S) / \operatorname{Inn}(S)$ : the set of $S$-conjugacy classes of morphisms from $P$ to $S$. Let $\mathcal{P}$ be the set of $S$-conjugacy classes of subgroups $\mathcal{F}$-conjugate to $P$, and let $\mathcal{P}_{\text {fn }} \subseteq \mathcal{P}$ be the subset of classes of subgroups fully normalized in $\mathcal{F}$. If $x \in \Omega_{0}$ and $\theta(x)=\varphi$, then for all $s \in S$ and $g \in P$,

$$
(1, s) \cdot x=(g, s \varphi(g)) \cdot x=\left(g, c_{s} \circ \varphi(g)\right) \cdot(1, s) \cdot x,
$$

and this shows that $\theta((1, s) \cdot x)=c_{s} \circ \theta(x)$. Thus $\theta$ induces a map

$$
\bar{\theta}:\left(\Omega / S_{2}\right)^{P}=\Omega_{0} / S_{2} \stackrel{\theta / S_{2}}{\longrightarrow} \operatorname{Rep}_{\mathcal{F}}(P, S) \stackrel{\operatorname{Im}(-)}{\longrightarrow} \mathcal{P},
$$

where $\operatorname{Rep}_{\mathcal{F}}(P, S)=\operatorname{Hom}_{\mathcal{F}}(P, S) / \operatorname{Inn}(S)$. Furthermore, $\left|\Omega / S_{2}\right| \equiv 1(\bmod p)$ by (c), and thus $\left|\left(\Omega / S_{2}\right)^{P}\right| \equiv 1(\bmod p)$. 
For each $P^{\prime}$ which is $\mathcal{F}$-conjugate to $P$, there are $|S| /\left|N_{S}\left(P^{\prime}\right)\right|$ distinct subgroups in the $S$-conjugacy class $\left[P^{\prime}\right]$. Hence there are

$$
\left|\operatorname{Aut}_{\mathcal{F}}(P)\right| \cdot|S| /\left|N_{S}\left(P^{\prime}\right)\right|
$$

elements of $\operatorname{Hom}_{\mathcal{F}}(P, S)$ whose image lies in $\left[P^{\prime}\right]$. Since each of these is the image of $k$ elements in $\Omega_{0}$, this shows that

$$
\left|\bar{\theta}^{-1}\left(\left[P^{\prime}\right]\right)\right|=k \cdot\left|\operatorname{Aut}_{\mathcal{F}}(P)\right| /\left|N_{S}\left(P^{\prime}\right)\right| .
$$

Thus $\left|N_{S}\left(P^{\prime}\right)\right||k \cdot| \operatorname{Aut}_{\mathcal{F}}(P) \mid$ for all $P^{\prime} \mathcal{F}$-conjugate to $P$, and so $\bar{\theta}^{-1}\left(\left[P^{\prime}\right]\right)$ has order a multiple of $p$ if $P^{\prime}$ is not fully normalized in $\mathcal{F}$ (if $\left[P^{\prime}\right] \in \mathcal{P} \backslash \mathcal{P}_{\text {fn }}$ ). Hence

$$
\left|\bar{\theta}^{-1}\left(\mathcal{P}_{\mathrm{fn}}\right)\right| \equiv\left|\left(\Omega / S_{2}\right)^{P}\right| \equiv 1 \quad(\bmod p) .
$$

So if we set $m=\left|N_{S}\left(P^{\prime}\right)\right|$ for $\left[P^{\prime}\right] \in \mathcal{P}_{\text {fn }}$ (i.e., the maximal value of $\left|N_{S}\left(P^{\prime}\right)\right|$ for $P^{\prime}$ $\mathcal{F}$-conjugate to $P$ ), then

$$
\left|\bar{\theta}^{-1}\left(\mathcal{P}_{\mathrm{fn}}\right)\right|=\left|\mathcal{P}_{\mathrm{fn}}\right| \cdot\left(\frac{k \cdot\left|\operatorname{Aut}_{\mathcal{F}}(P)\right|}{m}\right),
$$

and thus $\left|\mathcal{P}_{\text {fn }}\right|$ is prime to $p$.

Using a similar argument, one can also show that the $\operatorname{set}^{\operatorname{Rep}_{\mathcal{F}}^{\mathrm{fc}}}(P, S)$ of elements of $\operatorname{Rep}_{\mathcal{F}}(P, S)$ whose image is fully centralized also has order prime to $p$.

\section{The Fundamental group of $\mathcal{L}_{p}^{\wedge}$}

The purpose of this section is to give a simple description of the fundamental group of $|\mathcal{L}|_{p}^{\wedge}$, for any $p$-local finite group $(S, \mathcal{F}, \mathcal{L})$, purely in terms of the fusion system $\mathcal{F}$. The result is analogous to the (hyper-) focal subgroup theorem for finite groups, as we explain below.

In Section 1, we defined a functor $J: \mathcal{L}^{q} \rightarrow \mathcal{B}\left(\pi_{1}(|\mathcal{L}|)\right)$, for any $p$-local finite group $(S, \mathcal{F}, \mathcal{L})$, and a homomorphism $j=J \circ \delta_{S}$ from $S$ to $\pi_{1}(|\mathcal{L}|)$. Let $\tau: S \rightarrow \pi_{1}\left(|\mathcal{L}|_{p}^{\wedge}\right)$ be the composite of $j$ with the natural homomorphism from $\pi_{1}(|\mathcal{L}|)$ to $\pi_{1}\left(|\mathcal{L}|_{p}^{\wedge}\right)$.

In [BLO2, Proposition 1.12], we proved that $\tau: S \rightarrow \pi_{1}\left(|\mathcal{L}|_{p}^{\wedge}\right)$ is a surjection. In this section, we will show that $\operatorname{Ker}(\tau)$ is the hyperfocal subgroup of $\mathcal{F}$, defined by Puig [Pu3] (see also $\mathrm{Pu2}$ ).

Definition 2.1. For any saturated fusion system $\mathcal{F}$ over a $p$-group $S$, the hyperfocal subgroup of $\mathcal{F}$ is the normal subgroup of $S$ defined by

$$
O_{\mathcal{F}}^{p}(S)=\left\langle g^{-1} \alpha(g) \mid g \in P \leq S, \alpha \in O^{p}\left(\operatorname{Aut}_{\mathcal{F}}(P)\right)\right\rangle .
$$

We will prove, for any $p$-local finite group $(S, \mathcal{F}, \mathcal{L})$, that $\pi_{1}\left(|\mathcal{L}|_{p}^{\wedge}\right) \cong S / O_{\mathcal{F}}^{p}(S)$. This is motivated by Puig's hyperfocal theorem, and we will also need that theorem in order to prove it. Before stating Puig's theorem, we first recall the standard focal subgroup theorem. If $G$ is a finite group and $S \in \operatorname{Syl}_{p}(G)$, then this theorem says that $S \cap[G, G]$ (the focal subgroup) is the subgroup generated by all elements of the form $x^{-1} y$ for $x, y \in S$ which are $G$-conjugate (cf. [Go, Theorem 7.3.4] or [Suz2, $5.2 \cdot 8])$.

The quotient group $S /(S \cap[G, G])$ is isomorphic to the $p$-power torsion subgroup of $G /[G, G]$, and can thus be identified as a quotient group of the maximal $p$ group quotient $G / O^{p}(G)$. Since $G / O^{p}(G)$ is a $p$-group, $G=S \cdot O^{p}(G)$, and hence $G / O^{p}(G) \cong S /\left(S \cap O^{p}(G)\right)$. Hence $S \cap O^{p}(G) \leq S \cap[G, G]$. This subgroup 
$S \cap O^{p}(G)$ is what Puig calls the hyperfocal subgroup, and is described by the hyperfocal subgroup theorem in terms of $S$ and fusion.

For $S \in \operatorname{Syl}_{p}(G)$ as above, let $O_{G}^{p}(S)$ be the normal subgroup of $S$ defined by

$$
\begin{aligned}
O_{G}^{p}(S)=O_{\mathcal{F}_{S}(G)}^{p}(S) & =\left\langle g^{-1} \alpha(g) \mid g \in P \leq S, \alpha \in O^{p}\left(\operatorname{Aut}_{G}(P)\right)\right\rangle \\
& \left.=\langle[g, x]| g \in P \leq S, x \in N_{G}(P) \text { of order prime to } p\right\rangle .
\end{aligned}
$$

Lemma 2.2 ( $\mathrm{Pu} 2)$. Fix a prime $p$, a finite group $G$, and a Sylow subgroup $S \in$ $\operatorname{Syl}_{p}(G)$. Then $O_{G}^{p}(S)=S \cap O^{p}(G)$.

Proof. This is stated in $[\mathrm{Pu} 2, \S 1.1]$, but the proof is only sketched there, and so we elaborate on it here. Following standard notation, for any $P \leq S$ and any $A \leq$ $\operatorname{Aut}(P)$, we write $[P, A]=\left\langle x^{-1} \alpha(x) \mid x \in P, \alpha \in A\right\rangle$. Thus $O_{G}^{p}(S)$ is generated by the subgroups $\left[P, O^{p}\left(\operatorname{Aut}_{G}(P)\right)\right]$ for all $P \leq S$. It is clear that $O_{G}^{p}(S) \leq S \cap O^{p}(G)$; the problem is to prove the opposite inclusion.

Set $G_{*}=O^{p}(G)$ and $S_{*}=S \cap G_{*}$ for short. Then $\left[G_{*}, G_{*}\right]$ has index prime to $p$ in $G_{*}$, so it contains $S_{*}$. By the focal subgroup theorem (cf. Go, Theorem 7.3.4]), applied to $S_{*} \in \operatorname{Syl}_{p}\left(G_{*}\right), S_{*}$ is generated by all elements of the form $x^{-1} y$ for $x, y \in S_{*}$ which are $G_{*}$-conjugate. Combined with Alperin's fusion theorem (in Alperin's original version [A] or in the version of Theorem 1.5(a)), this implies that $S_{*}$ is generated by all subgroups $\left[P, N_{G_{*}}(P)\right]$ for $P \leq S_{*}$ such that $N_{S_{*}}(P) \in \operatorname{Syl}_{p}\left(N_{G_{*}}(P)\right)$. (This last condition is equivalent to $P$ being fully normalized in $\mathcal{F}_{S_{*}}\left(G_{*}\right)$.) Also, $N_{G_{*}}(P)$ is generated by $O^{p}\left(N_{G_{*}}(P)\right)$ and the Sylow subgroup $N_{S_{*}}(P)$, so $\left[P, N_{G_{*}}(P)\right]$ is generated by $\left[P, O^{p}\left(N_{G_{*}}(P)\right)\right] \leq O_{G}^{p}(S)$ and $\left[P, N_{S_{*}}(P)\right] \leq\left[S_{*}, S_{*}\right]$. Thus $S_{*}=\left\langle O_{G}^{p}(S),\left[S_{*}, S_{*}\right]\right\rangle$. Since $O_{G}^{p}(S)$ is normal in $S$ (hence also normal in $S_{*}$ ), this shows that $S_{*} / O_{G}^{p}(S)$ is equal to its commutator subgroup, which for a $p$-group is possible only if $S_{*} / O_{G}^{p}(S)$ is trivial, and hence $S_{*}=O_{G}^{p}(S)$.

By Proposition 1.14 the key to getting information about $\pi_{1}(|\mathcal{L}|)$ is to construct functors from $\mathcal{L}$ or $\mathcal{L}^{q}$ to $\mathcal{B}(\Gamma)$, for a group $\Gamma$, which send inclusions to the identity. The next lemma is our main inductive tool for doing this. Whenever $\alpha \in \operatorname{Mor}_{\mathcal{L}^{q}}\left(P, P^{\prime}\right)$ and $\beta \in \operatorname{Mor}_{\mathcal{L}^{q}}\left(Q, Q^{\prime}\right)$ are such that $P \leq Q, P^{\prime} \leq Q^{\prime}$, we write $\alpha=\left.\beta\right|_{P}$ to mean that $\alpha$ is the restriction of $\beta$ in the sense defined in Section 1; i.e., $\iota_{P^{\prime}}^{Q^{\prime}} \circ \alpha=\beta \circ \iota_{P}^{Q}$.

Lemma 2.3. Fix a p-local finite group $(S, \mathcal{F}, \mathcal{L})$, and let $\mathcal{L}^{q}$ be its associated quasicentric linking system. Assume that a compatible set of inclusions $\left\{\iota_{P}^{Q}\right\}$ has been chosen for $\mathcal{L}^{q}$. Let $\mathcal{H}_{0}$ be a set of $\mathcal{F}$-quasicentric subgroups of $S$ which is closed under $\mathcal{F}$-conjugacy and overgroups. Let $\mathcal{P}$ be an $\mathcal{F}$-conjugacy class of $\mathcal{F}$-quasicentric subgroups maximal among those not in $\mathcal{H}_{0}$, set $\mathcal{H}=\mathcal{H}_{0} \cup \mathcal{P}$, and let $\mathcal{L}^{\mathcal{H}_{0}} \subseteq \mathcal{L}^{\mathcal{H}} \subseteq \mathcal{L}^{q}$ be the full subcategories with these objects. Assume, for some group $\Gamma$, that

$$
\lambda_{0}: \mathcal{L}^{\mathcal{H}_{0}} \longrightarrow \mathcal{B}(\Gamma)
$$

is a functor which sends inclusions to the identity. Fix some $P \in \mathcal{P}$ which is fully normalized in $\mathcal{F}$, and fix a homomorphism $\lambda_{P}: \operatorname{Aut}_{\mathcal{L}^{q}}(P) \rightarrow \Gamma$. Assume that

(*) for all $P \supsetneqq Q \leq N_{S}(P)$ such that $Q$ is fully normalized in $N_{\mathcal{F}}(P)$, and for all $\alpha \in \operatorname{Aut}_{\mathcal{L}^{q}}(P)$ and $\beta \in \operatorname{Aut}_{\mathcal{L}^{q}}(Q)$ such that $\alpha=\left.\beta\right|_{P}, \lambda_{P}(\alpha)=\lambda_{0}(\beta)$.

Then there is a unique extension of $\lambda_{0}$ to a functor $\lambda: \mathcal{L}^{\mathcal{H}} \rightarrow \mathcal{B}(\Gamma)$ which sends inclusions to the identity, and such that $\lambda(\alpha)=\lambda_{P}(\alpha)$ for all $\alpha \in \operatorname{Aut}_{\mathcal{F}}(P)$. 
Proof. The uniqueness of the extension is an immediate consequence of Theorem 1.5(a) (Alperin's fusion theorem).

To prove the existence of the extension $\lambda$, we first show that $(*)$ implies the following (a prori stronger) statement:

(**) for all $Q, Q^{\prime} \leq S$ which strictly contain $P$, and for all $\beta \in \operatorname{Mor}_{\mathcal{L}^{q}}\left(Q, Q^{\prime}\right)$ and $\alpha \in \operatorname{Aut}_{\mathcal{L}^{q}}(P)$ such that $\alpha=\left.\beta\right|_{P}, \lambda_{P}(\alpha)=\lambda_{0}(\beta)$.

To see this, note first that it suffices to consider the case where $P$ is normal in $Q$ and $Q^{\prime}$. By assumption, $\pi(\beta)(P)=\pi(\alpha)(P)=P$, hence $\pi(\beta)\left(N_{Q}(P)\right) \leq N_{Q^{\prime}}(P)$, and therefore $\beta$ restricts to a morphism $\bar{\beta} \in \operatorname{Mor}_{\mathcal{L}^{q}}\left(N_{Q}(P), N_{Q^{\prime}}(P)\right)$ by Proposition 1.12(b) (applied with $\varphi=\beta \circ \iota_{N_{Q}(P)}^{Q}$ and $\psi=\iota_{N_{Q^{\prime}}(P)}^{Q^{\prime}}$ ). Since $N_{Q}(P), N_{Q^{\prime}}(P) \supsetneqq P$, by the induction hypothesis, $\lambda_{0}(\beta)=\lambda_{0}(\bar{\beta})$, so we are reduced to proving that $\lambda_{P}(\alpha)=\lambda_{0}(\bar{\beta})$.

Thus $\beta \in \operatorname{Mor}_{N_{\mathcal{L}}(P)}\left(Q, Q^{\prime}\right)$. We now apply Alperin's fusion theorem (Theorem [1.5(a)) to the morphism $\pi(\beta)$ in the fusion system $N_{\mathcal{F}}(P)$ (which is saturated by [BLO2, Proposition A.6]). Thus $\pi(\beta)=\varphi_{k} \circ \cdots \circ \varphi_{1}$, where each $\varphi_{i} \in \operatorname{Hom}_{N_{\mathcal{F}}(P)}\left(Q_{i-1}, Q_{i}\right)$ is the restriction to $Q_{i-1}$ of an automorphism $\widehat{\varphi}_{i} \in$ $\operatorname{Aut}_{N_{\mathcal{F}}(P)}\left(R_{i}\right)$, where $R_{i} \geq Q_{i-1}, Q_{i}$ is an $N_{\mathcal{F}}(P)$-centric subgroup of $N_{S}(P)$ which is fully normalized in $N_{\mathcal{F}}(P)$, and where $Q=Q_{0}$ and $Q^{\prime}=Q_{k}$. Each $R_{i}$ contains $P$, and hence is $\mathcal{F}$-centric by [BLO2, Lemma 6.2]. For each $\widehat{\varphi}_{i}$, also regarded as an automorphism in $\mathcal{F}$, we choose a lifting $\widehat{\beta}_{i} \in \operatorname{Aut}_{\mathcal{L}}\left(R_{i}\right)$, and let $\beta_{i} \in \operatorname{Mor}_{\mathcal{L}^{q}}\left(Q_{i-1}, Q_{i}\right)$ be its restriction. By $(\mathrm{A})_{q}, \beta=\beta_{k} \circ \cdots \circ \beta_{1} \circ \delta_{Q}(g)$ for some $g \in C_{S}(Q)$, and hence

$$
\begin{aligned}
\lambda_{0}(\beta) & =\lambda_{0}\left(\beta_{k}\right) \cdots \lambda_{0}\left(\beta_{1}\right) \cdot \lambda_{0}\left(\delta_{Q}(g)\right)=\lambda_{0}\left(\widehat{\beta}_{k}\right) \cdots \lambda_{0}\left(\widehat{\beta}_{1}\right) \cdot \lambda_{0}\left(\delta_{N_{S}(P)}(g)\right) \\
& =\lambda_{P}\left(\left.\widehat{\beta}_{k}\right|_{P}\right) \cdots \lambda_{P}\left(\left.\widehat{\beta}_{1}\right|_{P}\right) \cdot \lambda_{P}\left(\operatorname{Id}_{P}\right)=\lambda_{P}\left(\left.\beta\right|_{P}\right)=\lambda_{P}(\alpha),
\end{aligned}
$$

where the third equality follows from $(*)$. This finishes the proof of $(* *)$.

We can now extend $\lambda$ to be defined on all morphisms in $\mathcal{L}^{\mathcal{H}}$ not in $\mathcal{L}^{\mathcal{H}_{0}}$. Fix such a morphism $\varphi \in \operatorname{Mor}_{\mathcal{L}^{q}}\left(P_{1}, Q\right)$. Set $P_{2}=\pi(\varphi)\left(P_{1}\right) \leq Q$; then $P_{1}, P_{2} \in \mathcal{P}$, and $\varphi=\iota_{P_{2}}^{Q} \circ \varphi^{\prime}$ for some unique $\varphi^{\prime} \in \operatorname{Iso}_{\mathcal{L}^{q}}\left(P_{1}, P_{2}\right)$. By Lemma 1.3 (and then lifting to the linking category), there are isomorphisms $\bar{\varphi}_{i} \in \operatorname{Iso}_{\mathcal{L}^{q}}\left(N_{S}\left(P_{i}\right), N_{i}\right)$, for some $N_{i} \leq N_{S}(P)$ containing $P$, which restrict to isomorphisms $\varphi_{i} \in \operatorname{Iso}_{\mathcal{L}^{q}}\left(P_{i}, P\right)$. Set $\psi=\varphi_{2} \circ \varphi^{\prime} \circ \varphi_{1}^{-1} \in \operatorname{Aut}_{\mathcal{L}^{q}}(P)$. We have thus decomposed $\varphi^{\prime}$ as the composite $\varphi_{2}^{-1} \circ \psi \circ \varphi_{1}$, and can now define

$$
\lambda(\varphi)=\lambda\left(\varphi^{\prime}\right)=\lambda_{0}\left(\bar{\varphi}_{2}\right)^{-1} \cdot \lambda_{P}(\psi) \cdot \lambda_{0}\left(\bar{\varphi}_{1}\right) .
$$

Now let $\varphi^{\prime}=\left(\varphi_{2}^{\prime}\right)^{-1} \circ \psi^{\prime} \circ \varphi_{1}^{\prime}$ be another such decomposition, where $\varphi_{i}^{\prime}$ is the restriction of $\bar{\varphi}_{i}^{\prime} \in \operatorname{Iso}_{\mathcal{L}^{q}}\left(N_{S}\left(P_{i}\right), N_{i}^{\prime}\right)$. We thus have a commutative diagram

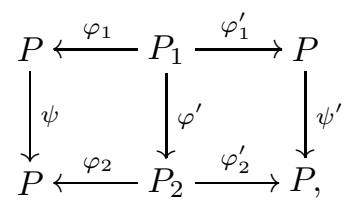

where for each $i, \varphi_{i}$ and $\varphi_{i}^{\prime}$ are restrictions of isomorphisms $\bar{\varphi}_{i}$ and $\bar{\varphi}_{i}^{\prime}$ defined on $N_{S}\left(P_{i}\right)$. To see that the two decompositions give the same value of $\lambda(\varphi)$, it remains 
to show that

$$
\lambda_{P}\left(\psi^{\prime}\right) \cdot \lambda_{0}\left(\bar{\varphi}_{1}^{\prime} \circ\left(\bar{\varphi}_{1}\right)^{-1}\right)=\lambda_{0}\left(\bar{\varphi}_{2}^{\prime} \circ\left(\bar{\varphi}_{2}\right)^{-1}\right) \cdot \lambda_{P}(\psi) .
$$

This holds since $\lambda_{0}\left(\bar{\varphi}_{i}^{\prime} \circ\left(\bar{\varphi}_{i}\right)^{-1}\right)=\lambda_{P}\left(\varphi_{i}^{\prime} \circ\left(\varphi_{i}\right)^{-1}\right)$ by $(* *)$.

We have now defined $\lambda$ on all morphisms in $\mathcal{L}^{\mathcal{H}}$, and it sends inclusion morphisms to the identity by construction. By construction, $\lambda$ sends composites to products, and thus the proof is complete.

Lemma 2.3 provides the induction step when proving the following proposition, which is the main result needed to compute $\pi_{1}\left(|\mathcal{L}|_{p}^{\wedge}\right)$.

Proposition 2.4. Fix a p-local finite group $(S, \mathcal{F}, \mathcal{L})$, and let $\mathcal{L}^{q}$ be its associated quasicentric linking system. Assume that a compatible set of inclusions $\left\{\iota_{P}^{Q}\right\}$ has been chosen for $\mathcal{L}^{q}$. Then there is a unique functor

$$
\lambda: \mathcal{L}^{q} \longrightarrow \mathcal{B}\left(S / O_{\mathcal{F}}^{p}(S)\right)
$$

which sends inclusions to the identity, and such that $\lambda\left(\delta_{S}(g)\right)=g$ for all $g \in S$.

Proof. The functor $\lambda$ will be constructed inductively, using Lemma 2.3 Let $\mathcal{H}_{0} \subseteq$ $\mathrm{Ob}\left(\mathcal{L}^{q}\right)$ be a subset (possibly empty) which is closed under $\mathcal{F}$-conjugacy and overgroups. Let $\mathcal{P}$ be an $\mathcal{F}$-conjugacy class of $\mathcal{F}$-quasicentric subgroups maximal among those not in $\mathcal{H}_{0}$, set $\mathcal{H}=\mathcal{H}_{0} \cup \mathcal{P}$, and let $\mathcal{L}^{\mathcal{H}_{0}} \subseteq \mathcal{L}^{\mathcal{H}} \subseteq \mathcal{L}^{q}$ be the full subcategories with these objects. Assume that

$$
\lambda_{0}: \mathcal{L}^{\mathcal{H}_{0}} \longrightarrow \mathcal{B}\left(S / O_{\mathcal{F}}^{p}(S)\right)
$$

has already been constructed, such that $\lambda_{0}\left(\delta_{S}(g)\right)=g$ for all $g \in S$ (if $S \in \mathcal{H}_{0}$ ), and such that $\lambda_{0}$ sends inclusions to the identity.

Fix $P \in \mathcal{P}$ which is fully normalized in $\mathcal{F}$, and let $\delta_{P, P}: N_{S}(P) \longrightarrow \operatorname{Aut}_{\mathcal{L}^{q}}(P)$ be the homomorphism of Proposition 1.13. Then $\operatorname{Im}\left(\delta_{P, P}\right)$ is a Sylow $p$-subgroup of $\operatorname{Aut}_{\mathcal{L}^{q}}(P)$, since $\operatorname{Aut}_{S}(P) \in \operatorname{Syl}_{p}\left(\operatorname{Aut}_{\mathcal{F}}(P)\right)$ by axiom $(\mathrm{I})$. We identify $N_{S}(P)$ as a subgroup of $\operatorname{Aut}_{\mathcal{L}^{q}}(P)$ to simplify notation. Then

$$
\left.\operatorname{Aut}_{\mathcal{L}^{q}}(P) / O^{p} \operatorname{Aut}_{\mathcal{L}^{q}}(P)\right) \cong N_{S}(P) /\left(N_{S}(P) \cap O^{p}\left(\operatorname{Aut}_{\mathcal{L}^{q}}(P)\right)\right)=N_{S}(P) / N_{0},
$$

where by Lemma 2.2. $N_{0}$ is the subgroup generated by all commutators $[g, x]$ for

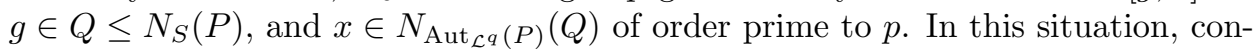
jugation by $x$ lies in $\operatorname{Aut}_{\mathcal{F}}(Q)$ by Proposition $1.13(\mathrm{~d})$, and thus $[g, x]=g \cdot c_{x}(g)^{-1} \in$ $O_{\mathcal{F}}^{p}(S)$. We conclude that $N_{0} \leq O_{\mathcal{F}}^{p}(S)$, and hence that the inclusion of $N_{S}(P)$ into $S$ extends to a homomorphism

$$
\lambda_{P}: \operatorname{Aut}_{\mathcal{L}^{q}}(P) \longrightarrow S / O_{\mathcal{F}}^{p}(S) .
$$

We claim that condition $(*)$ in Lemma 2.3 holds for $\lambda_{0}$ and $\lambda_{P}$. To see this, fix $P \supsetneqq Q \leq S$ such that $P \triangleleft Q$ and $Q$ is fully normalized in $N_{\mathcal{F}}(P)$, and fix $\alpha \in \operatorname{Aut}_{\mathcal{L}^{q}}(P)$ and $\beta \in \operatorname{Aut}_{\mathcal{L}^{q}}(Q)$ such that $\alpha=\left.\beta\right|_{P}$. We must show that $\lambda_{P}(\alpha)=$ $\lambda_{0}(\beta)$. Upon replacing $\alpha$ by $\alpha^{k}$ and $\beta$ by $\beta^{k}$ for some appropriate $k \equiv 1(\bmod p)$, we can assume that both automorphisms have order a power of $p$. Since $Q$ is fully normalized, $\operatorname{Aut}_{N_{S}(P)}(Q)$ is a Sylow subgroup of $\operatorname{Aut}_{N_{\mathcal{F}}(P)}(Q)$ ). Hence (since any two Sylow $p$-subgroups of a finite group $G$ are conjugate by an element of $O^{p}(G)$ ), there is an automorphism $\bar{\gamma} \in O^{p}\left(\operatorname{Aut}_{\left.N_{\mathcal{L}^{q}(P)}(Q)\right)}\right.$ such that $\bar{\gamma} \beta \bar{\gamma}^{-1}=\bar{\delta}_{Q}(g)$ for some $g \in N_{S}(Q) \cap N_{S}(P)$. In particular, $\lambda_{0}(\bar{\gamma})=1$ since it is a composite of automorphisms of order prime to $p$. Set $\gamma=\left.\bar{\gamma}\right|_{P}$; then $\gamma \in O^{p}\left(\operatorname{Aut}_{\mathcal{L}^{q}}(P)\right)$ and 
hence $\lambda_{P}(\gamma)=1$. Using axiom $(\mathrm{C})_{q}$ and Lemma 1.12, we see that $\gamma \alpha \gamma^{-1}=\bar{\delta}_{P}(g)$, and thus (since $\lambda_{P}(\gamma)=1$ and $\lambda_{0}(\bar{\gamma})=1$ ) that

$$
\lambda_{0}(\beta)=\lambda_{0}\left(\bar{\delta}_{Q}(g)\right)=g=\lambda_{P}\left(\bar{\delta}_{P}(g)\right)=\lambda_{P}(\alpha) .
$$

Thus, by Lemma 2.3, we can extend $\lambda_{0}$ to a functor defined on $\mathcal{L}^{\mathcal{H}}$. Upon continuing this procedure, we obtain a functor $\lambda$ defined on all of $\mathcal{L}^{q}$.

For any finite group $G, \pi_{1}\left(B G_{p}^{\wedge}\right) \cong G / O^{p}(G)$. (This is implicit in [BK, §VII.3], and shown explicitly in BLO1, Proposition A.2].) Hence our main result in this section can be thought of as the hyperfocal theorem for $p$-local finite groups.

Theorem 2.5 (The hyperfocal subgroup theorem for $p$-local finite groups). Let $(S, \mathcal{F}, \mathcal{L})$ be a p-local finite group. Then

$$
\pi_{1}\left(|\mathcal{L}|_{p}^{\wedge}\right) \cong S / O_{\mathcal{F}}^{p}(S)
$$

More precisely, the natural map $\tau: S \rightarrow \pi_{1}\left(|\mathcal{L}|_{p}^{\wedge}\right)$ is surjective, and $\operatorname{Ker}(\tau)=$ $O_{\mathcal{F}}^{p}(S)$.

Proof. Let $\lambda: \mathcal{L} \longrightarrow \mathcal{B}\left(S / O_{\mathcal{F}}^{p}(S)\right)$ be the functor of Proposition 2.4, and let $|\lambda|$ be the induced map between geometric realizations. Since $\left|\mathcal{B}\left(S / O_{\mathcal{F}}^{p}(S)\right)\right|$ is the classifying space of a finite $p$-group and hence $p$-complete, $|\lambda|$ factors through the $p$-completion $|\mathcal{L}|_{p}^{\wedge}$. Consider the following commutative diagram:

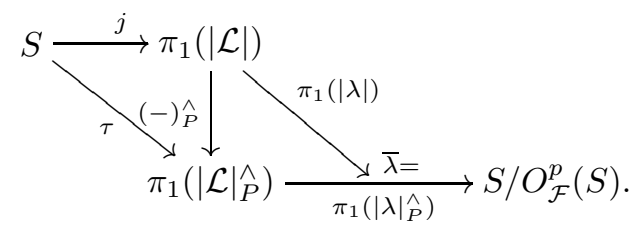

Here, $\tau$ is surjective by BLO2, Proposition 1.12]. Also, by construction, the composite $\bar{\lambda} \circ \tau=\pi_{1}(|\lambda|) \circ j$ is the natural projection. Thus $\operatorname{Ker}(\tau) \leq O_{\mathcal{F}}^{p}(S)$, and it remains to show the opposite inclusion.

Fix $g \in P \leq S$ and $\alpha \in \operatorname{Aut}_{\mathcal{F}}(P)$ such that $\alpha$ has order prime to $p$; we want to show that $g^{-1} \alpha(g) \in \operatorname{Ker}(\tau)$. Since $\operatorname{Ker}(\tau)$ is closed under $\mathcal{F}$-conjugacy (Proposition 1.14(d)), for any $\varphi \in \operatorname{Iso}_{\mathcal{F}}\left(P, P^{\prime}\right), g^{-1} \alpha(g)$ is in $\operatorname{Ker}(\tau)$ if $\varphi\left(g^{-1} \alpha(g)\right)$ is in $\operatorname{Ker}(\tau)$. In particular, since $\varphi\left(g^{-1} \alpha(g)\right)=\varphi(g)^{-1} \cdot\left(\varphi \alpha \varphi^{-1}\right)(\varphi(g))$, we can assume that $P$ is fully centralized in $\mathcal{F}$. Then, upon extending $\alpha$ to an automorphism of $P \cdot C_{S}(P)$, which can also be assumed to have order prime to $p$ (replace it by an appropriate power if necessary), we can assume that $P$ is $\mathcal{F}$-centric. In this case, by Proposition $1.14(\mathrm{c}), j(g)$ and $j(\alpha(g))$ are conjugate in $\pi_{1}(|\mathcal{L}|)$ by an element of order prime to $p$, and hence are equal in $\pi_{1}\left(|\mathcal{L}|_{p}^{\wedge}\right)$ since this is a $p$-group. This shows that $g^{-1} \alpha(g) \in \operatorname{Ker}(\tau)$, and finishes the proof of the theorem.

The following result, which will be useful in Section 5, is of a similar nature, but much more elementary.

Proposition 2.6. Fix a p-local finite group $(S, \mathcal{F}, \mathcal{L})$, and let $\mathcal{L}^{q}$ be its associated quasicentric linking system. Then the induced maps

$$
\pi_{1}(|\mathcal{L}|) \longrightarrow \pi_{1}\left(\left|\mathcal{F}^{c}\right|\right) \quad \text { and } \quad \pi_{1}\left(\left|\mathcal{L}^{q}\right|\right) \longrightarrow \pi_{1}\left(\left|\mathcal{F}^{q}\right|\right)
$$

are surjective, and their kernels are generated by elements of p-power order. 
Proof. We prove this for $\left|\mathcal{L}^{q}\right|$ and $\left|\mathcal{F}^{q}\right|$; a similar argument applies to $|\mathcal{L}|$ and $\left|\mathcal{F}^{c}\right|$. Recall from Section 1 that we can regard $\pi_{1}\left(\left|\mathcal{L}^{q}\right|\right)$ as the group generated by the loops $J(\alpha)$, for $\alpha \in \operatorname{Mor}\left(\mathcal{L}^{q}\right)$, with relations given by composition of morphisms and making inclusion morphisms equal to 1 . In a similar way, we regard $\pi_{1}\left(\left|\mathcal{F}^{q}\right|\right)$ as being generated by loops $J(\varphi)$ for $\varphi \in \operatorname{Mor}\left(\mathcal{F}^{q}\right)$. Since every morphism $\alpha \in$ $\operatorname{Hom}_{\mathcal{F}^{q}}(P, Q)$ has a lifting to a morphism of $\mathcal{L}^{q}$, the map $\pi_{\#}: \pi_{1}\left(\left|\mathcal{L}^{q}\right|\right) \rightarrow \pi_{1}\left(\left|\mathcal{F}^{q}\right|\right)$ induced by the projection functor $\pi: \mathcal{L}^{q} \rightarrow \mathcal{F}^{q}$ is an epimorphism.

Write $J C$ for the normal subgroup of $\pi_{1}\left(\left|\mathcal{L}^{q}\right|\right)$ generated by the loops $j(g)=$ $J\left(\delta_{P}(g)\right)$, for all $\mathcal{F}$-quasicentric subgroups $P \leq S$ and all $g \in C_{S}(P)$. In particular, $J C$ is generated by elements of $p$-power order. Since $\pi_{\#}(j(g))=1, J C$ is contained in the kernel of $\pi_{\#}$, and we have a factorization $\widehat{\pi}_{\#}: \pi_{1}\left(\left|\mathcal{L}^{q}\right|\right) / J C \rightarrow \pi_{1}\left(\left|\mathcal{F}^{q}\right|\right)$.

Define an inverse $s: \pi_{1}\left(\left|\mathcal{F}^{q}\right|\right) \rightarrow \pi_{1}\left(\left|\mathcal{L}^{q}\right|\right) / J C$ as follows. Given a morphism $\alpha \in \operatorname{Hom}_{\mathcal{F} q}(P, Q)$, choose a lifting $\bar{\alpha}$ in $\mathcal{L}^{q}$, and set $s(\alpha)=[J(\bar{\alpha})]$. If $\bar{\alpha}, \bar{\alpha}^{\prime} \in$ $\operatorname{Hom}_{\mathcal{L}^{q}}(P, Q)$ are two liftings of $\alpha$, then there is an isomorphism $\chi: P^{\prime} \rightarrow P$ in $\mathcal{L}^{q}$ where $P^{\prime}$ is fully centralized, and an element $g \in C_{S}\left(P^{\prime}\right)$, such that $\bar{\alpha}^{\prime}=$ $\bar{\alpha} \circ\left(\chi \circ \delta_{P^{\prime}}(g) \circ \chi^{-1}\right)$. Since $\chi \circ \delta_{P^{\prime}}(g) \circ \chi^{-1}$ represents a loop that belongs to $J C,[J(\bar{\alpha})]=\left[J\left(\bar{\alpha}^{\prime}\right)\right]$. Thus the definition of $s$ does not depend on the choice of $\bar{\alpha}$. It remains to show that $s$ preserves the relations among the generators. But we clearly have that $s\left(\left[J\left(\operatorname{incl}_{P}^{Q}\right)\right]\right)=\left[J\left(\iota_{P}^{Q}\right)\right]=1$. Also, if $\alpha$ and $\beta$ are composable morphisms of $\mathcal{F}^{q}$, and $\bar{\alpha}$ and $\bar{\beta}$ are liftings to $\mathcal{L}^{q}$, then $\bar{\alpha} \circ \bar{\beta}$ is a lifting of $\alpha \circ \beta$, and hence $s(\alpha) s(\beta)=[J(\bar{\alpha})][J(\bar{\beta})]=[J(\bar{\alpha} \circ \bar{\beta})]=s(\alpha \circ \beta)$.

This shows that $\pi_{\#}$ induces an isomorphim $\pi_{1}\left(\left|\mathcal{L}^{q}\right|\right) / J C \cong \pi_{1}\left(\left|\mathcal{F}^{q}\right|\right)$.

\section{Subsystems with $p$-Solvable Quotient}

In this section, we prove some general results about subsystems of saturated fusion systems and $p$-local finite subgroups: subsystems with $p$-group quotient or quotient of order prime to $p$. These will then be used in the next two sections to prove some more specific theorems.

It will be convenient in this section to write " $p$ '-group" for a finite group of order prime to $p$. Recall that a $p$-solvable group is a group $G$ with normal series $1=H_{0} \triangleleft H_{1} \triangleleft \cdots \triangleleft H_{k}=G$ such that each $H_{i} / H_{i-1}$ is a $p$-group or a $p^{\prime}$-group. As one consequence of the results of this section, we show that for any $p$-local finite group $(S, \mathcal{F}, \mathcal{L})$, and any homomorphism $\theta$ from $\pi_{1}(|\mathcal{L}|)$ to a finite $p$-solvable group, there is another $p$-local finite group $\left(S_{0}, \mathcal{F}_{0}, \mathcal{L}_{0}\right)$ such that $\left|\mathcal{L}_{0}\right|$ is homotopy equivalent to the covering space of $|\mathcal{L}|$ with fundamental group $\operatorname{Ker}(\theta)$.

We start with some definitions. Recall that for any finite group $G, O^{p^{\prime}}(G)$ and $O^{p}(G)$ are the smallest normal subgroups of $G$ of index prime to $p$ and of $p$-power index, respectively. Equivalently, $O^{p^{\prime}}(G)$ is the subgroup generated by elements of $p$-power order in $G$, and $O^{p}(G)$ is the subgroup generated by elements of order prime to $p$ in $G$.

We want to identify the fusion subsystems of a given fusion system which are analogous to subgroups of $G$ which contain $O^{p^{\prime}}(G)$ or $O^{p}(G)$. This motivates the following definitions.

Definition 3.1. Let $\mathcal{F}$ be a saturated fusion system over a $p$-group $S$. Let $\left(S^{\prime}, \mathcal{F}^{\prime}\right) \subseteq(S, \mathcal{F})$ be a saturated fusion subsystem; i.e., $S^{\prime} \leq S$ is a subgroup, and $\mathcal{F}^{\prime} \subseteq \mathcal{F}$ is a subcategory which is a saturated fusion system over $S^{\prime}$. 
(a) $\left(S^{\prime}, \mathcal{F}^{\prime}\right)$ is of p-power index in $(S, \mathcal{F})$ if

$$
S^{\prime} \geq O_{\mathcal{F}}^{p}(S) \quad \text { and } \quad \operatorname{Aut}_{\mathcal{F}^{\prime}}(P) \geq O^{p}\left(\operatorname{Aut}_{\mathcal{F}}(P)\right)
$$

for all $P \leq S^{\prime}$. Equivalently, a saturated fusion subsystem $\mathcal{F}^{\prime} \subseteq \mathcal{F}$ over $S^{\prime} \geq O_{\mathcal{F}}^{p}(S)$ has $p$-power index if it contains all $\mathcal{F}$-automorphisms of order prime to $p$ of subgroups of $S^{\prime}$.

(b) $\left(S^{\prime}, \mathcal{F}^{\prime}\right)$ is of index prime to $p$ in $(S, \mathcal{F})$ if

$$
S^{\prime}=S \quad \text { and } \quad \operatorname{Aut}_{\mathcal{F}^{\prime}}(P) \geq O^{p^{\prime}}\left(\operatorname{Aut}_{\mathcal{F}}(P)\right)
$$

for all $P \leq S$. Equivalently, a saturated fusion subsystem $\mathcal{F}^{\prime} \subseteq \mathcal{F}$ over $S$ has index prime to $p$ if it contains all $\mathcal{F}$-automorphisms of $p$-power order.

This terminology has been chosen for simplicity. Subsystems "of $p$-power index" or "of index prime to $p$ " are really analogous to subgroups $H \leq G$ which contain normal subgroups of $G$ of $p$-power index or index prime to $p$, respectively. For example, if $\mathcal{F}$ is a saturated fusion system over $S$, and $\mathcal{F}^{\prime} \subseteq \mathcal{F}$ is the fusion system of $S$ itself (i.e., the minimal fusion system over $S$ ), then $\mathcal{F}^{\prime}$ is not in general a subsystem of index prime to $p$ under the above definition, despite the inclusion $\mathcal{F}^{\prime} \subseteq \mathcal{F}$ being analogous to the inclusion of a Sylow $p$-subgroup in a group.

Over the next three sections, we will classify all saturated fusion subsystems of $p$-power index, or of index prime to $p$, in a given saturated fusion system. In both cases, there will be a minimal such subsystem, denoted $O^{p}(\mathcal{F})$ or $O^{p^{\prime}}(\mathcal{F})$; and the saturated fusion subsystems of the given type will be in bijective correspondence with the subgroups of a given $p$-group or $p^{\prime}$-group.

The following terminology will be useful for describing some of the categories we have to work with.

Definition 3.2. Fix a finite $p$-group $S$.

(a) A restrictive category over $S$ is a category $\mathcal{F}$ such that $\mathrm{Ob}(\mathcal{F})$ is the set of subgroups of $S$, such that all morphisms in $\mathcal{F}$ are group monomorphisms between the subgroups, and with the following additional property: for each $P^{\prime} \leq P \leq S$ and $Q^{\prime} \leq Q \leq S$, and each $\varphi \in \operatorname{Hom}_{\mathcal{F}}(P, Q)$ such that $\varphi\left(P^{\prime}\right) \leq Q^{\prime},\left.\varphi\right|_{P^{\prime}} \in \operatorname{Hom}_{\mathcal{F}}\left(P^{\prime}, Q^{\prime}\right)$.

(b) A restrictive category $\mathcal{F}$ over $S$ is normalized by an automorphism $\alpha \in$ $\operatorname{Aut}_{\mathcal{F}}(S)$ if for each $P, Q \leq S$, and each $\varphi \in \operatorname{Hom}_{\mathcal{F}}(P, Q), \alpha \varphi \alpha^{-1} \in$ $\operatorname{Hom}_{\mathcal{F}}(\alpha(P), \alpha(Q))$.

(c) For any restrictive category $\mathcal{F}$ over $S$ and any subgroup $A \leq \operatorname{Aut}(S),\langle\mathcal{F}, A\rangle$ is the smallest restrictive category over $S$ which contains $\mathcal{F}$ together with all automorphisms in $A$ and their restrictions.

By definition, any restrictive category is required to contain all inclusion homomorphisms (restrictions of $\operatorname{Id}_{S}$ ). The main difference between a restrictive category over $S$ and a fusion system over $S$ is that the restrictive subcategory need not contain $\mathcal{F}_{S}(S)$.

When $\mathcal{F}$ is a fusion system over $S$, then an automorphism $\alpha \in \operatorname{Aut}(S)$ normalizes $\mathcal{F}$ if and only if it is fusion preserving in the sense used in BLO1. For the purposes of this paper, it will be convenient to use both terms, in different situations.

We next define the followingsubcategories of a given fusion system $\mathcal{F}$. 
Definition 3.3. Let $\mathcal{F}$ be any fusion system over a $p$-group $S$.

(a) $O_{*}^{p}(\mathcal{F}) \subseteq \mathcal{F}$ denotes the smallest restrictive subcategory of $\mathcal{F}$ whose morphism set contains $O^{p}\left(\operatorname{Aut}_{\mathcal{F}}(P)\right)$ for all subgroups $P \leq S$.

(b) $O_{*}^{p^{\prime}}(\mathcal{F}) \subseteq \mathcal{F}$ denotes the smallest restrictive subcategory of $\mathcal{F}$ whose morphism set contains $O^{p^{\prime}}\left(\operatorname{Aut}_{\mathcal{F}}(P)\right)$ for all subgroups $P \leq S$.

By definition, for subgroups $P, Q$, a morphism $\varphi \in \operatorname{Hom}_{\mathcal{F}}(P, Q)$ lies in $O_{*}^{p^{\prime}}(\mathcal{F})$ if and only if it is a composite of morphisms which are restrictions of elements of $O^{p^{\prime}}\left(\operatorname{Aut}_{\mathcal{F}}(R)\right)$ for subgroups $R \leq S$. Morphisms in $O_{*}^{p}(\mathcal{F})$ are described in a similar way.

The subcategory $O_{*}^{p}(\mathcal{F})$ is not, in general, a fusion system - and this is why we had to define restrictive categories. The subcategory $O_{*}^{p^{\prime}}(\mathcal{F})$ is always a fusion system (since $\operatorname{Aut}_{S}(P) \leq O^{p^{\prime}}\left(\operatorname{Aut}_{\mathcal{F}}(P)\right)$ for all $P \leq S$ ), but it is not, in general, saturated. The subscripts " $*$ " have been put in as a reminder of these facts, and as a contrast with the notation $O^{p}(\mathcal{F})$ and $O^{p^{\prime}}(\mathcal{F})$ which will be used to denote certain minimal saturated fusion systems.

We now check some of the basic properties of the subcategories $O_{*}^{p}(\mathcal{F})$ and $O_{*}^{p^{\prime}}(\mathcal{F})$, stated in terms of these definitions.

Lemma 3.4. The following hold for any fusion system $\mathcal{F}$ over a p-group $S$ :

(a) $O_{*}^{p}(\mathcal{F})$ and $O_{*}^{p^{\prime}}(\mathcal{F})$ are normalized by $\operatorname{Aut}_{\mathcal{F}}(S)$.

(b) If $\mathcal{F}$ is saturated, then $\mathcal{F}=\left\langle O_{*}^{p}(\mathcal{F}), \operatorname{Aut}_{\mathcal{F}}(S)\right\rangle=\left\langle O_{*}^{p^{\prime}}(\mathcal{F})\right.$, $\left.\operatorname{Aut}_{\mathcal{F}}(S)\right\rangle$.

(c) If $\mathcal{F}^{\prime} \subseteq \mathcal{F}$ is any restrictive subcategory normalized by $\operatorname{Aut}_{\mathcal{F}}(S)$ and such that $\mathcal{F}=\left\langle\mathcal{F}^{\prime}, \operatorname{Aut}_{\mathcal{F}}(S)\right\rangle$, then for each $P, Q \leq S$ and $\varphi \in \operatorname{Hom}_{\mathcal{F}}(P, Q)$, there are morphisms $\alpha \in \operatorname{Aut}_{\mathcal{F}}(S), \varphi^{\prime} \in \operatorname{Hom}_{\mathcal{F}^{\prime}}(\alpha(P), Q)$, and $\varphi^{\prime \prime} \in$ $\operatorname{Hom}_{\mathcal{F}^{\prime}}\left(P, \alpha^{-1} Q\right)$, such that $\varphi=\left.\varphi^{\prime} \circ \alpha\right|_{P}=\alpha \circ \varphi^{\prime \prime}$.

Proof. To simplify notation in the following proofs, for $\alpha \in \operatorname{Aut}_{\mathcal{F}}(S)$, we write $\alpha$ in composites of morphisms between subgroups of $S$, rather than specifying the appropriate restriction each time.

(a) Set $\mathcal{F}^{\prime}=O_{*}^{p}(\mathcal{F})$ or $O_{*}^{p^{\prime}}(\mathcal{F})$. Let $\alpha \in \operatorname{Aut}_{\mathcal{F}}(S)$ and let $\varphi \in \operatorname{Hom}_{\mathcal{F}^{\prime}}(P, Q)$. We must show that $\alpha \varphi \alpha^{-1} \in \operatorname{Hom}_{\mathcal{F}^{\prime}}(\alpha(P), \alpha(Q))$. By definition of $\mathcal{F}^{\prime}$, there are subgroups

$$
P=P_{0}, P_{1}, \ldots, P_{k}=\varphi(P) \leq Q,
$$

subgroups $\bar{P}_{1}, \ldots, \bar{P}_{k}$, and automorphisms $\chi_{i} \in O^{p}\left(\operatorname{Aut}_{\mathcal{F}}\left(\bar{P}_{i}\right)\right)\left(\right.$ if $\left.\mathcal{F}^{\prime}=O_{*}^{p}(\mathcal{F})\right)$ or $\chi_{i} \in O^{p^{\prime}}\left(\operatorname{Aut}_{\mathcal{F}}\left(\bar{P}_{i}\right)\right)\left(\right.$ if $\left.\mathcal{F}^{\prime}=O_{*}^{p^{\prime}}(\mathcal{F})\right)$, such that $P_{i-1}, P_{i} \leq \bar{P}_{i}, \chi_{i}\left(P_{i-1}\right)=P_{i}$, and

$$
\varphi=\operatorname{incl}_{P_{k}}^{Q} \circ\left(\left.\chi_{k}\right|_{P_{k-1}}\right) \circ \cdots \circ\left(\left.\chi_{2}\right|_{P_{1}}\right) \circ\left(\left.\chi_{1}\right|_{P_{0}}\right) \text {. }
$$

Let $P_{i}^{\prime}=\alpha\left(P_{i}\right), \bar{P}_{i}^{\prime}=\alpha\left(\bar{P}_{i}\right)$, and $\chi_{i}^{\prime}=\alpha \chi_{i} \alpha^{-1} \in O^{p}\left(\operatorname{Aut}_{\mathcal{F}}\left(\bar{P}_{i}^{\prime}\right)\right)$ or $O^{p^{\prime}}\left(\operatorname{Aut}_{\mathcal{F}}\left(\bar{P}_{i}^{\prime}\right)\right)$. Then

$$
\alpha \varphi \alpha^{-1}=\operatorname{incl}_{P_{k}^{\prime}}^{\alpha(Q)} \circ\left(\left.\chi_{k}^{\prime}\right|_{P_{k-1}^{\prime}}\right) \circ \cdots \circ\left(\left.\chi_{2}^{\prime}\right|_{P_{1}^{\prime}}\right) \circ\left(\left.\chi_{1}^{\prime}\right|_{P_{0}^{\prime}}\right)
$$

is in $\operatorname{Hom}_{\mathcal{F}^{\prime}}(\alpha(P), \alpha(Q))$, as required.

(b1) Now assume that $\mathcal{F}$ is saturated. We show here that

$$
\mathcal{F}=\left\langle O_{*}^{p^{\prime}}(\mathcal{F}), \operatorname{Aut}_{\mathcal{F}}(S)\right\rangle ;
$$

i.e., that each $\varphi \in \operatorname{Mor}(\mathcal{F})$ is a composite of morphisms in $O_{*}^{p^{\prime}}(\mathcal{F})$ and in $\operatorname{Aut}_{\mathcal{F}}(S)$. By Alperin's fusion theorem for saturated fusion systems (Theorem 1.5(a)), it suffices to prove this when $\varphi \in \operatorname{Aut}_{\mathcal{F}}(P)$ for some $P \leq S$ which is $\mathcal{F}$-centric and fully 
normalized. The result clearly holds if $P=S$. So we can assume that $P \supsetneqq S$, and also assume inductively that the lemma holds for every automorphism of any subgroup $P^{\prime} \leq S$ such that $\left|P^{\prime}\right|>|P|$.

Consider the subgroup

$$
K=\varphi \operatorname{Aut}_{S}(P) \varphi^{-1}=\left\{\varphi c_{g} \varphi^{-1} \in \operatorname{Aut}_{\mathcal{F}}(P) \mid g \in N_{S}(P)\right\} .
$$

Then $K$ is a $p$-subgroup of $\operatorname{Aut}_{\mathcal{F}}(P)$, and hence $K \leq \operatorname{Aut}_{O_{*}^{p^{\prime}}(\mathcal{F})}(P)$. Since $P$ is fully normalized in $\mathcal{F}$, $\operatorname{Aut}_{S}(P) \in \operatorname{Syl}_{p}\left(\operatorname{Aut}_{\mathcal{F}}(P)\right)$. Thus $K$ and $\operatorname{Aut}_{S}(P)$ are both Sylow $p$-subgroups of Aut ${ }_{O_{*}^{p^{\prime}}(\mathcal{F})}(P)$, and there is some $\chi \in \operatorname{Aut}_{O_{*}^{p^{\prime}}(\mathcal{F})}(P)$ such that $\chi K \chi^{-1} \leq \operatorname{Aut}_{S}(P)$. In other words,

$$
N_{\chi \varphi} \stackrel{\text { def }}{=}\left\{g \in N_{S}(P) \mid(\chi \varphi) c_{g}(\chi \varphi)^{-1} \in \operatorname{Aut}_{S}(P)\right\}=N_{S}(P) .
$$

So by condition (II) in Definition 1.2 $\chi \circ \varphi$ can be extended to a homomorphism $\psi \in \operatorname{Hom}_{\mathcal{F}}\left(N_{S}(P), S\right)$. By the induction hypothesis (and since $N_{S}(P) \supsetneqq P$ ), $\psi$, and hence $\chi \circ \varphi$, are composites of morphisms in $N_{\mathcal{F}}(S)$ and in $O_{*}^{p^{\prime}}(\mathcal{F})$. Also, $\chi \in \operatorname{Aut}_{O_{*}^{p^{\prime}}(\mathcal{F})}(P)$ by assumption, and hence $\varphi$ is a composite of morphisms in these two subcategories.

(b2) To see that $\mathcal{F}=\left\langle O_{*}^{p}(\mathcal{F})\right.$, $\left.\operatorname{Aut}_{\mathcal{F}}(S)\right\rangle$ when $\mathcal{F}$ is saturated, it again suffices to restrict to the case where $\varphi \in \operatorname{Aut}_{\mathcal{F}}(P)$ for some $P \leq S$ which is $\mathcal{F}$-centric and fully normalized. But in this case, $\operatorname{Aut}_{\mathcal{F}}(P)$ is generated by its Sylow $p$-subgroup $\operatorname{Aut}_{S}(P) \leq \operatorname{Aut}_{N_{\mathcal{F}}(S)}(P)$, together with $O^{p}\left(\operatorname{Aut}_{\mathcal{F}}(P)\right) \leq \operatorname{Aut}_{O_{*}^{p}(\mathcal{F})}(P)$.

(c) Since $\mathcal{F}=\left\langle\mathcal{F}^{\prime}, \operatorname{Aut}_{\mathcal{F}}(S)\right\rangle$, every morphism in $\mathcal{F}$ is a composite of morphisms in $\mathcal{F}^{\prime}$ and restrictions of automorphisms in $\operatorname{Aut}_{\mathcal{F}}(S)$. Assume $\varphi \in \operatorname{Hom}_{\mathcal{F}}(P, Q)$ is the composite

$$
P=P_{0} \stackrel{\alpha_{0}}{\longrightarrow} Q_{0} \stackrel{\varphi_{1}}{\longrightarrow} P_{1} \stackrel{\alpha_{1}}{\longrightarrow} Q_{1} \longrightarrow \ldots \longrightarrow P_{n} \stackrel{\alpha_{n}}{\longrightarrow} Q_{n}=Q
$$

where $\alpha_{i} \in \operatorname{Aut}_{\mathcal{F}}(S), \alpha_{i}\left(P_{i}\right)=Q_{i}$, and $\varphi_{i} \in \operatorname{Hom}_{\mathcal{F}^{\prime}}\left(Q_{i-1}, P_{i}\right)$. Write $\alpha_{j, i}=$ $\alpha_{j} \cdots \alpha_{i}$ for any $i \leq j$, and set $\alpha=\alpha_{n, 0}=\alpha_{n} \cdots \alpha_{0}$. Then

$$
\begin{aligned}
\varphi & =\alpha \circ\left(\alpha_{n-1,0}{ }^{-1} \varphi_{n} \alpha_{n-1,0}\right) \cdots\left(\alpha_{0,0}{ }^{-1} \varphi_{1} \alpha_{0,0}\right) \\
& =\left(\alpha_{n, n} \varphi_{n} \alpha_{n, n}{ }^{-1}\right) \cdots\left(\alpha_{n, 1} \varphi_{1} \alpha_{n, 1}{ }^{-1}\right) \circ \alpha ;
\end{aligned}
$$

where each $\alpha_{n, i} \varphi_{i} \alpha_{n, i}{ }^{-1}$ and each $\alpha_{i, 0}{ }^{-1} \varphi_{i+1} \alpha_{i, 0}$ is a morphism in $\mathcal{F}^{\prime}$ since $\mathcal{F}^{\prime}$ is normalized by $\operatorname{Aut}_{\mathcal{F}}(S)$.

The following lemma will also be needed later.

Lemma 3.5. Let $\mathcal{F}$ be a saturated fusion system over a p-group S. Fix a normal subgroup $S_{0} \triangleleft S$ which is strongly $\mathcal{F}$-closed; i.e., no element of $S_{0}$ is $\mathcal{F}$-conjugate to any element of $S \backslash S_{0}$. Let $\left(S_{0}, \mathcal{F}_{0}\right)$ be a saturated fusion subsystem of $(S, \mathcal{F})$. Then for any $P \leq S$ which is $\mathcal{F}$-centric and $\mathcal{F}$-radical, $P \cap S_{0}$ is $\mathcal{F}_{0}$-centric.

Proof. Assume $P \leq S$ is $\mathcal{F}$-centric and $\mathcal{F}$-radical, and set $P_{0}=P \cap S_{0}$ for short. Choose a subgroup $P_{0}^{\prime} \leq S_{0}$ which is $\mathcal{F}$-conjugate to $P_{0}$ and fully normalized in $\mathcal{F}$. In particular, by $(\mathrm{I}), P_{0}^{\prime}$ is fully centralized in $\mathcal{F}$. By Lemma 1.3, there is $\varphi \in \operatorname{Hom}_{\mathcal{F}}\left(N_{S}\left(P_{0}\right), N_{S}\left(P_{0}^{\prime}\right)\right)$ such that $\varphi\left(P_{0}\right)=P_{0}^{\prime}$. Set $P^{\prime}=\varphi(P)$; thus $P^{\prime}$ is $\mathcal{F}$ conjugate to $P$ (so $P^{\prime}$ is also $\mathcal{F}$-centric and $\mathcal{F}$-radical), and $P_{0}^{\prime}=P^{\prime} \cap S_{0}$ since $S_{0}$ is strongly closed. For any $P_{0}^{\prime \prime} \leq S_{0}$ which is $\mathcal{F}_{0}$-conjugate to $P_{0}$ (hence $\mathcal{F}$-conjugate to $\left.P_{0}^{\prime}\right)$, there is a morphism $\psi \in \operatorname{Hom}_{\mathcal{F}}\left(P_{0}^{\prime \prime} \cdot C_{S}\left(P_{0}^{\prime \prime}\right), P_{0}^{\prime} \cdot C_{S}\left(P_{0}^{\prime}\right)\right)$ such that $\psi\left(P_{0}^{\prime \prime}\right)=P_{0}^{\prime}$ 
(by axiom (II)), and then $\psi\left(C_{S_{0}}\left(P_{0}^{\prime \prime}\right)\right) \leq C_{S_{0}}\left(P_{0}^{\prime}\right)$. So if $C_{S_{0}}\left(P_{0}^{\prime}\right)=Z\left(P_{0}^{\prime}\right)$, then $C_{S_{0}}\left(P_{0}^{\prime \prime}\right)=Z\left(P_{0}^{\prime \prime}\right)$ for all $P_{0}^{\prime \prime}$ which is $\mathcal{F}_{0}$-conjugate to $P_{0}$, and $P_{0}$ is $\mathcal{F}_{0}$-centric.

We are thus reduced to showing that $C_{S_{0}}\left(P_{0}^{\prime}\right)=Z\left(P_{0}^{\prime}\right)$; and without loss of generality, we can assume that $P^{\prime}=P$ and $P_{0}^{\prime}=P_{0}$. Since $S_{0}$ is strongly closed, every $\alpha \in \operatorname{Aut}_{\mathcal{F}}(P)$ leaves $P_{0}$ invariant. Let $\operatorname{Aut}_{\mathcal{F}}^{0}(P) \leq \operatorname{Aut}_{\mathcal{F}}(P)$ be the subgroup of elements which induce the identity on $P_{0}$ and on $P / P_{0}$. This is a normal subgroup of $\operatorname{Aut}_{\mathcal{F}}(P)$ since all elements of $\operatorname{Aut}_{\mathcal{F}}(P)$ leave $P_{0}$ invariant, and is also a $p$ subgroup by Lemma 1.15. Thus $\operatorname{Aut}_{\mathcal{F}}^{0}(P) \leq O_{p}\left(\operatorname{Aut}_{\mathcal{F}}(P)\right)$, and hence $\operatorname{Aut}_{\mathcal{F}}^{0}(P) \leq$ $\operatorname{Inn}(P)$ since $P$ is $\mathcal{F}$-radical.

We want to show that $C_{S_{0}}\left(P_{0}\right)=Z\left(P_{0}\right)$. Fix any $x \in C_{S_{0}}\left(P_{0}\right)$, and assume first that the coset $x \cdot Z\left(P_{0}\right) \in C_{S_{0}}\left(P_{0}\right) / Z\left(P_{0}\right)$ is fixed by the conjugation action of $P$. Thus $x \in S_{0},\left[x, P_{0}\right]=1$, and $[x, P] \leq Z\left(P_{0}\right)$, so $c_{x} \in \operatorname{Aut}_{\mathcal{F}}^{0}(P) \leq \operatorname{Inn}(P)$, and $x g \in C_{S}(P)$ for some $g \in P$. Since $P$ is $\mathcal{F}$-centric, this implies that $x g \in$ $P$, so $x \in C_{S_{0}}\left(P_{0}\right) \cap P=Z\left(P_{0}\right)$. In other words, $\left[C_{S_{0}}\left(P_{0}\right) / Z\left(P_{0}\right)\right]^{P}=1$, so $C_{S_{0}}\left(P_{0}\right) / Z\left(P_{0}\right)=1$, and thus $C_{S_{0}}\left(P_{0}\right)=Z\left(P_{0}\right)$.

The motivation for the next definition comes from considering the situation which arises when one is given a saturated fusion system $\mathcal{F}$ with an associated quasicentric linking system $\mathcal{L}^{q}$, and a functor $\mathcal{L}^{q} \rightarrow \mathcal{B}(\Gamma)$ which sends inclusions to the identity (or equivalently a homomorphism from $\pi_{1}\left(\left|\mathcal{L}^{q}\right|\right.$ ) to $\Gamma$ ) for some group $\Gamma$. Such a functor is equivalent to a function $\bar{\Theta}: \operatorname{Mor}\left(\mathcal{L}^{q}\right) \rightarrow \Gamma$ which sends composites to products and sends inclusions to the identity; and for any $H \leq \Gamma$, $\bar{\Theta}^{-1}(H)=\operatorname{Mor}\left(\mathcal{L}_{H}^{q}\right)$ for some subcategory $\mathcal{L}_{H}^{q} \subseteq \mathcal{L}^{q}$ with the same objects. Let $\mathcal{F}_{H}^{q} \subseteq \mathcal{F}^{q}$ be the image of $\mathcal{L}_{H}^{q}$ under the canonical projection; then in some sense (to be made precise later), $\mathcal{F}_{H}^{q}$ is a subsystem of $\mathcal{F}^{q}$ of index $[\Gamma: H]$.

What we now need is to make sense of such "inverse image subcategories" of the fusion system $\mathcal{F}$, when we are not assuming that we have an associated linking system. Let $\mathfrak{S u b}(\Gamma)$ denote the set of nonempty subsets of $\Gamma$. Given a function $\bar{\Theta}$ as above, there is an obvious associated function $\Theta: \operatorname{Mor}\left(\mathcal{F}^{q}\right) \rightarrow \mathfrak{S u b}(\Gamma)$, which sends a morphism $\alpha \in \operatorname{Mor}\left(\mathcal{F}^{q}\right)$ to $\bar{\Theta}\left(\pi^{-1}(\alpha)\right)$. Here, $\pi$ denotes the natural projection from $\mathcal{L}^{q}$ to $\mathcal{F}^{q}$. Moreover, $\bar{\Theta}$ also induces a homomorphism $\theta=\bar{\Theta} \circ \delta_{S}$ from $S$ to $\Gamma$. The maps $\Theta$ and $\theta$ are closely related to each other, and satisfy certain properties, none of which depend on the existence (or choice) of a quasicentric linking system associated to $\mathcal{F}$. In fact, we will see that the data encoded in such a pair of functions, if they satisfy the appropriate conditions, suffices to describe precisely what is meant by "inverse image subcategories" of fusion systems; and to show that under certain restrictions, these categories are (or generate) saturated fusion subsystems.

Definition 3.6. Let $\mathcal{F}$ be a saturated fusion system over a $p$-group $S$, and let $\mathcal{F}_{0} \subseteq \mathcal{F}^{q}$ be any full subcategory such that $\operatorname{Ob}\left(\mathcal{F}_{0}\right)$ is closed under $\mathcal{F}$-conjugacy. A fusion mapping triple for $\mathcal{F}_{0}$ consists of a triple $(\Gamma, \theta, \Theta)$, where $\Gamma$ is a group, $\theta: S \longrightarrow \Gamma$ is a homomorphism, and

$$
\Theta: \operatorname{Mor}\left(\mathcal{F}_{0}\right) \longrightarrow \mathfrak{S u b}(\Gamma)
$$

is a map which satisfies the following conditions for all subgroups $P, Q, R \leq S$ which lie in $\mathcal{F}_{0}$ :

(i) For all $P \stackrel{\varphi}{\longrightarrow} Q \stackrel{\psi}{\longrightarrow} R$ in $\mathcal{F}_{0}$, and all $x \in \Theta(\psi), \Theta(\psi \varphi)=x \cdot \Theta(\varphi)$.

(ii) If $P$ is fully centralized in $\mathcal{F}$, then $\Theta\left(\operatorname{Id}_{P}\right)=\theta\left(C_{S}(P)\right)$. 
(iii) If $\varphi=c_{g} \in \operatorname{Hom}_{\mathcal{F}}(P, Q)$, where $g \in N_{S}(P, Q)$, then $\theta(g) \in \Theta(\varphi)$.

(iv) For all $\varphi \in \operatorname{Hom}_{\mathcal{F}}(P, Q)$, all $x \in \Theta(\varphi)$, and all $g \in P, x \theta(g) x^{-1}=\theta(\varphi(g))$.

For any fusion mapping triple $(\Gamma, \theta, \Theta)$ and any $H \leq \Gamma$, we let $\mathcal{F}_{H}^{\bullet} \subseteq \mathcal{F}$ be the smallest restrictive subcategory which contains all $\varphi \in \operatorname{Mor}\left(\mathcal{F}^{q}\right)$ such that $\Theta(\varphi) \cap$ $H \neq \emptyset$. Let $\mathcal{F}_{H} \subseteq \mathcal{F}_{H}^{\bullet}$ be the full subcategory whose objects are the subgroups of $\theta^{-1}(H)$.

When $\theta$ is the trivial homomorphism (which is always the case when $\Gamma$ is a $p^{\prime}$-group), then a fusion mapping triple $(\Gamma, \theta, \Theta)$ on a subcategory $\mathcal{F}_{0} \subseteq \mathcal{F}^{q}$ is equivalent to a functor from $\mathcal{F}_{0}$ to $\mathcal{B}(\Gamma)$; i.e., $\Theta(\varphi)$ contains just one element for all $\varphi \in \operatorname{Mor}\left(\mathcal{F}_{0}\right)$. By (i), it suffices to show this for identity morphisms; and by (ii), $\left|\Theta\left(\operatorname{Id}_{P}\right)\right|=1$ if $P$ is fully centralized. For arbitrary $P$, it then follows from (i), together with the assumption (included in the definition of $\mathfrak{S u b}(\Gamma)$ ) that $\Theta(\varphi) \neq \emptyset$ for all $\varphi$.

The following additional properties of fusion mapping triples will be needed.

Lemma 3.7. Fix a saturated fusion system $\mathcal{F}$ over a p-group $S$, let $\mathcal{F}_{0}$ be a full subcategory such that $\mathrm{Ob}\left(\mathcal{F}_{0}\right)$ is closed under $\mathcal{F}$-conjugacy, and let $(\Gamma, \Theta, \theta)$ be a fusion mapping triple for $\mathcal{F}_{0}$. Then the following hold for all $P, Q, R \in \operatorname{Ob}\left(\mathcal{F}_{0}\right)$ :

(v) $\Theta\left(\operatorname{Id}_{P}\right)$ is a subgroup of $\Gamma$, and $\Theta$ restricts to a homomorphism

$$
\Theta_{P}: \operatorname{Aut}_{\mathcal{F}}(P) \rightarrow N_{\Gamma}\left(\Theta\left(\operatorname{Id}_{P}\right)\right) / \Theta\left(\operatorname{Id}_{P}\right) .
$$

Thus $\Theta_{P}(\alpha)=\Theta(\alpha)$ (as a coset of $\Theta\left(\operatorname{Id}_{P}\right)$ ) for all $\alpha \in \operatorname{Aut}_{\mathcal{F}}(P)$.

(vi) For all $P \stackrel{\varphi}{\longrightarrow} Q \stackrel{\psi}{\longrightarrow} R$ in $\mathcal{F}_{0}$, and all $x \in \Theta(\varphi), \Theta(\psi \varphi) \supseteq \Theta(\psi) \cdot x$, with equality if $\varphi(P)=Q$. In particular, if $P \leq Q$, then $\Theta\left(\left.\psi\right|_{P}\right) \supseteq \Theta(\psi)$.

(vii) Assume $S \in \operatorname{Ob}\left(\mathcal{F}_{0}\right)$. Then for any $\varphi \in \operatorname{Hom}_{\mathcal{F}}(P, Q)$, any $\alpha \in \operatorname{Aut}_{\mathcal{F}}(S)$, and any $x \in \Theta(\alpha), \Theta\left(\alpha \varphi \alpha^{-1}\right)=x \Theta(\varphi) x^{-1}$, where

$$
\alpha \varphi \alpha^{-1} \in \operatorname{Hom}_{\mathcal{F}}(\alpha(P), \alpha(Q)) .
$$

Proof. (v) By (i), for any $\alpha, \beta \in \operatorname{Aut}_{\mathcal{F}}(P)$ and any $x \in \Theta(\alpha), \Theta(\alpha \beta)=x \cdot \Theta(\beta)$. When applied with $\alpha=\beta=\operatorname{Id}_{P}$, this shows that $\Theta\left(\operatorname{Id}_{P}\right)$ is a subgroup of $\Gamma$. (Note here that $\Theta\left(\operatorname{Id}_{P}\right) \neq \emptyset$ by definition of $\mathfrak{S} \mathfrak{u} \mathfrak{b}(\Gamma)$.) When applied with $\beta=\alpha^{-1}$, this shows that $x^{-1} \in \Theta\left(\alpha^{-1}\right)$ if $x \in \Theta(\alpha)$. Hence $\Theta(\alpha)=x \cdot \Theta\left(\operatorname{Id}_{P}\right)$ implies that $\Theta\left(\alpha^{-1}\right)=\Theta\left(\operatorname{Id}_{P}\right) \cdot x^{-1}$ and $\Theta\left(\alpha^{-1}\right)=x^{-1} \cdot \Theta\left(\operatorname{Id}_{P}\right)$, and thus that each $\Theta(\alpha)$ is a right coset as well as a left coset. Thus $\Theta(\alpha) \subseteq N_{\Gamma}\left(\Theta\left(\operatorname{Id}_{P}\right)\right)$ for all $\alpha \in \operatorname{Aut}_{\mathcal{F}}(P)$, and the induced map $\Theta_{P}$ is clearly a homomorphism.

(vi) By (i), $\Theta(\psi \varphi) \supseteq \Theta(\psi) \cdot \Theta(\varphi)$ for any pair of composable morphisms $\varphi, \psi$ in $\mathcal{F}_{0}$. In particular, $\Theta(\psi \varphi) \supseteq \Theta(\psi) \cdot x$ if $x \in \Theta(\varphi)$. If $\varphi$ is an isomorphism, then $1 \in \Theta\left(\operatorname{Id}_{P}\right)=x \cdot \Theta\left(\varphi^{-1}\right)$ by $(\mathrm{v})$ and (i), so $x^{-1} \in \Theta\left(\varphi^{-1}\right)$. This gives the inclusions

$$
\Theta(\psi)=\Theta\left(\psi \varphi \varphi^{-1}\right) \supseteq \Theta(\psi \varphi) \cdot x^{-1} \supseteq \Theta(\psi) x x^{-1},
$$

and hence these are both equalities. The last statement is the special case where $P \leq Q$ and $\varphi=\operatorname{incl}_{P}^{Q} ; 1 \in \Theta\left(\operatorname{incl}_{P}^{Q}\right)$ by (iii).

(vii) For $x \in \Theta(\alpha), \Theta(\alpha \varphi)=x \cdot \Theta(\varphi)=\Theta\left(\alpha \varphi \alpha^{-1}\right) \cdot x$ by (i) and (vi).

We are now ready to prove the main result about fusion mapping triples.

Proposition 3.8. Let $\mathcal{F}$ be a saturated fusion system over a finite p-group $S$. Let $(\Gamma, \theta, \Theta)$ be any fusion mapping triple on $\mathcal{F}^{q}$, where $\Gamma$ is a p-group or a $p^{\prime}$-group, and

$$
\theta: S \longrightarrow \Gamma \quad \text { and } \quad \Theta: \operatorname{Mor}\left(\mathcal{F}^{q}\right) \longrightarrow \mathfrak{S u b}(\Gamma) \text {. }
$$


Then the following hold for any subgroup $H \leq \Gamma$, where we set $S_{H}=\theta^{-1}(H)$ :

(a) $\mathcal{F}_{H}$ is a saturated fusion system over $S_{H}$.

(b) If $\Gamma$ is a p-group, then a subgroup $P \leq S_{H}$ is $\mathcal{F}_{H}$-quasicentric if and only if it is $\mathcal{F}$-quasicentric. Also, $\mathcal{F}_{1}^{\bullet} \supseteq O_{*}^{p}(\mathcal{F})$.

(c) If $\Gamma$ is a $p^{\prime}$-group, then $S_{H}=S$. A subgroup $P \leq S$ is $\mathcal{F}_{H}$-centric (fully centralized in $\mathcal{F}_{H}$, fully normalized in $\mathcal{F}_{H}$ ) if and only if it is $\mathcal{F}$-centric (fully centralized in $\mathcal{F}$, fully normalized in $\mathcal{F}$ ). Also, $\mathcal{F}_{1} \bullet \supseteq O_{*}^{p^{\prime}}(\mathcal{F})$.

Proof. Throughout the proof, (i)-(vii) refer to the conditions in Definition 3.6 and Lemma 3.7. If $X$ is any set of morphisms of $\mathcal{F}$, then we let $\Theta(X)$ be the union of the sets $\Theta(\alpha)$ for $\alpha \in X$. Condition (v) implies that for any $P \leq S$ and any subgroup $A \leq \operatorname{Aut}_{\mathcal{F}}(P), \Theta(A)$ is a subgroup of $\Gamma$.

We first prove the following two additional properties of these subcategories:

(1) For each pair of subgroups $P, Q \leq S$, and each $\varphi \in \operatorname{Hom}_{\mathcal{F}}(P, Q)$, there are $Q^{\prime} \leq S, \varphi^{\prime} \in \operatorname{Hom}_{\mathcal{F}_{i}}\left(P, Q^{\prime}\right)$, and $\alpha \in \operatorname{Aut}_{\mathcal{F}}(S)$ such that $\alpha\left(Q^{\prime}\right)=Q$ and $\varphi=\left(\left.\alpha\right|_{Q^{\prime}}\right) \circ \varphi^{\prime}$.

(2) For all $P \leq S$ there exists $P^{\prime} \leq S$ which is fully normalized in $\mathcal{F}$, and $\varphi \in \operatorname{Hom}_{\mathcal{F}_{\mathbf{i}}}\left(N_{S}(P), N_{S}\left(P^{\prime}\right)\right)$ such that $\varphi(P)=P^{\prime}$.

By (vii), for any $\alpha \in \operatorname{Aut}_{\mathcal{F}}(S)$, any $x \in \Theta(\alpha)$, and any $\varphi \in \operatorname{Hom}_{\mathcal{F}}(P, Q)$, $\Theta\left(\alpha \varphi \alpha^{-1}\right)=x \Theta(\varphi) x^{-1}$. In particular, $\Theta\left(\alpha \varphi \alpha^{-1}\right)=1$ if and only if $\Theta(\varphi)=1$, and thus $\mathcal{F}_{1}^{\bullet}$ is normalized by $\operatorname{Aut}_{\mathcal{F}}(S)$.

Let $O_{*}^{p^{\prime}}(\mathcal{F})^{c} \subseteq O_{*}^{p^{\prime}}(\mathcal{F})$ and $O_{*}^{p}(\mathcal{F})^{c} \subseteq O_{*}^{p}(\mathcal{F})$ be the full subcategories whose objects are the $\mathcal{F}$-centric subgroups of $S$. By (v), for each $\mathcal{F}$-quasicentric subgroup $P \leq S, \operatorname{Aut}_{\mathcal{F}_{\mathbf{i}}}(P)=\operatorname{Ker}\left(\Theta_{P}\right)$, where $\Theta_{P}$ is a homomorphism to a subquotient of $\Gamma$. Hence $\operatorname{Aut}_{\mathcal{F}_{\mathbf{P}}}(P)$ contains $O^{p^{\prime}}\left(\operatorname{Aut}_{\mathcal{F}}(P)\right)$ if $\Gamma$ is a $p^{\prime}$-group or $O^{p}\left(\operatorname{Aut}_{\mathcal{F}}(P)\right)$ if $\Gamma$ is a $p$-group. This shows that $\mathcal{F}_{1}^{\bullet}$ contains either $O_{*}^{p^{\prime}}(\mathcal{F})^{c}$ (if $\Gamma$ is a $p^{\prime}$-group) or $O_{*}^{p}(\mathcal{F})^{c}$ (if $\Gamma$ is a $p$-group). Thus $\mathcal{F}^{c} \subseteq\left\langle\mathcal{F}_{1}^{\bullet}, \operatorname{Aut}_{\mathcal{F}}(S)\right\rangle$ by Lemma 3.4(b), and so $\mathcal{F}=\left\langle\mathcal{F}_{1}^{\bullet}, \operatorname{Aut}_{\mathcal{F}}(S)\right\rangle$ since $\mathcal{F}$ is the smallest restrictive category over $S$ which contains $\mathcal{F}^{c}$ by Theorem 1.5(a) (Alperin's fusion theorem). Point (1) now follows from Lemma 3.4 (c).

To see (2), recall that by Lemma 1.3] if $P^{\prime \prime}$ is any subgroup $\mathcal{F}$-conjugate to $P$ and fully normalized in $\mathcal{F}$, then there is a morphism $\varphi^{\prime} \in \operatorname{Hom}_{\mathcal{F}}\left(N_{S}(P), N_{S}\left(P^{\prime \prime}\right)\right)$ such that $\varphi^{\prime}(P)=P^{\prime \prime}$. By $(1), \varphi^{\prime}=\alpha \circ \varphi$ for some $\alpha \in \operatorname{Aut}_{\mathcal{F}}(S)$ and some $\varphi \in \operatorname{Hom}_{\mathcal{F}_{\mathbf{i}}}\left(N_{S}(P), N_{S}\left(\alpha^{-1}\left(P^{\prime \prime}\right)\right)\right)$. Also, the subgroup $P^{\prime}=\alpha^{-1}\left(P^{\prime \prime}\right)$ is fully normalized in $\mathcal{F}$ since $P^{\prime \prime}$ is.

(b) Assume $\Gamma$ is a $p$-group, and fix $H \leq \Gamma$. Consider the following set of subgroups of $S_{H}$ :

$$
\begin{aligned}
\mathcal{Q}_{H}=\left\{P \leq S_{H} \mid \forall P^{\prime} \mathcal{F}_{H^{-} \text {-conjugate to } P, \forall P^{\prime} \leq Q \leq P^{\prime} \cdot C_{S_{H}}\left(P^{\prime}\right),}\right. \\
\left.\quad \forall \alpha \in \operatorname{Aut}_{\mathcal{F}_{H}}(Q) \text { such that }\left.\alpha\right|_{P^{\prime}}=\mathrm{Id},|\alpha| \text { is a power of } p\right\} .
\end{aligned}
$$

This set clearly contains all $\mathcal{F}_{H}$-centric subgroups of $S_{H}$. By Lemma 1.6, if $\mathcal{F}_{H}$ is saturated, then $\mathcal{Q}_{H}$ is precisely the set of $\mathcal{F}_{H}$-quasicentric subgroups. We prove (b) here with " $\mathcal{F}_{H}$-quasicentric" replaced by "element of $\mathcal{Q}_{H}$ ". This is all that will be needed for the proof that $\mathcal{F}_{H}$ is saturated; and once that is shown then (b) (in its original form) will follow. 
Assume $P \leq S_{H}$ and $P \notin \mathcal{Q}_{H}$. Fix $P^{\prime}$ which is $\mathcal{F}_{H}$-conjugate to $P, Q \leq$ $P^{\prime} \cdot C_{S_{H}}\left(P^{\prime}\right)$ which contains $P^{\prime}$, and $\operatorname{Id}_{Q} \neq \alpha \in \operatorname{Aut}_{\mathcal{F}_{H}}(Q)$ of order prime to $p$, such that $\left.\alpha\right|_{P^{\prime}}=\operatorname{Id}_{P^{\prime}}$. Then $P^{\prime}$, and hence $P$, is not $\mathcal{F}$-quasicentric by Lemma 1.6(a).

We have shown that if $P$ is $\mathcal{F}$-quasicentric, then $P \in \mathcal{Q}_{H}$, and it remains to check the converse. Assume $P$ is not $\mathcal{F}$-quasicentric: fix $P^{\prime} \leq Q \leq P^{\prime} \cdot C_{S}\left(P^{\prime}\right)$ and $\alpha \in \operatorname{Aut}_{\mathcal{F}}(Q)$ as in Lemma 1.6(b). In particular, $Q$ is $\mathcal{F}$-centric, and hence $\mathcal{F}$-quasicentric. Set $Q_{1}=Q \cap S_{1}$ (where $S_{1}=\theta^{-1}(1)$ ). Since $1 \in \Theta(\alpha)$ by (v) (and since $|\alpha|$ is prime to $p), \theta(g)=\theta(\alpha(g))$ for all $g \in Q$ by (iv), and thus $\alpha\left(Q_{1}\right)=Q_{1}$ and (since $g^{-1} \alpha(g) \in \operatorname{Ker}(\theta)=S_{1}$ for $g \in Q$ ) $\alpha$ induces the identity on $Q / Q_{1}$. Since $|\alpha|$ is not a power of $p$, it cannot be the identity on both $Q_{1}$ and $Q / Q_{1}$ (Lemma 1.15), and hence $\left.\alpha\right|_{Q_{1}} \neq \operatorname{Id}_{Q_{1}}$. Thus $P^{\prime} \leq P^{\prime} Q_{1} \leq P^{\prime} \cdot C_{S_{H}}\left(P^{\prime}\right),\left.\alpha\right|_{P^{\prime} Q_{1}}$ is a nontrivial automorphism of $P^{\prime} Q_{1}$ of order prime to $p$ whose restriction to $P^{\prime}$ is the identity.

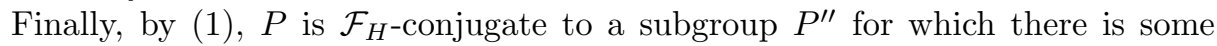
$\varphi \in \operatorname{Iso}_{\mathcal{F}}\left(P^{\prime}, P^{\prime \prime}\right)$ which is the restriction of some $\bar{\varphi} \in \operatorname{Aut}_{\mathcal{F}}(S)$. Since $P \leq S_{H}$ and is $\mathcal{F}_{H}$-conjugate to $P^{\prime \prime}, P^{\prime \prime} \leq S_{H}$ by (iv) (applied with $\alpha \in \operatorname{Iso}_{\mathcal{F}_{H}}\left(P, P^{\prime \prime}\right)$ and $x \in$ $\Theta(\alpha) \cap H)$. Set $Q^{\prime \prime}=\bar{\varphi}\left(P^{\prime} Q_{1}\right)=P^{\prime \prime} \cdot \bar{\varphi}\left(Q_{1}\right) \leq S_{H}$ and $\alpha^{\prime \prime}=\bar{\varphi} \alpha \bar{\varphi}^{-1} \in \operatorname{Aut}_{\mathcal{F}}\left(Q^{\prime \prime}\right)$. Since $\Gamma$ is a $p$-group and $\left|\alpha^{\prime \prime}\right|$ is prime to $p,(\mathrm{v})$ implies that $\Theta\left(\alpha^{\prime \prime}\right)=\Theta\left(\operatorname{Id}_{Q}\right)$, and hence that $1 \in \Theta\left(\alpha^{\prime \prime}\right)$ and hence $\alpha^{\prime \prime} \in \operatorname{Aut}_{\mathcal{F}_{H}}\left(Q^{\prime \prime}\right)$. But then $P^{\prime \prime} \notin \mathcal{Q}_{H}$, and hence $P \notin \mathcal{Q}_{H}$.

It remains to show that $\mathcal{F}_{1}^{\bullet} \supseteq O_{*}^{p}(\mathcal{F})$; i.e., to show that

$$
\operatorname{Aut}_{\mathcal{F}_{\mathbf{i}}}(P) \geq O^{p}\left(\operatorname{Aut}_{\mathcal{F}}(P)\right)
$$

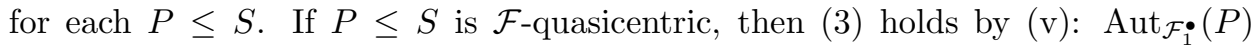
is the kernel of a homomorphism from $\operatorname{Aut}_{\mathcal{F}}(P)$ to a $p$-group. If $P$ is not $\mathcal{F}$ quasicentric but is fully centralized in $\mathcal{F}$, then every automorphism $\alpha \in \operatorname{Aut}_{\mathcal{F}}(P)$ of order prime to $p$ extends to an automorphism $\bar{\alpha} \in \operatorname{Aut}_{\mathcal{F}}\left(P \cdot C_{S}(P)\right.$ ), which (after replacing it by an appropriate power) can also be assumed to have order prime to $p$, and hence in $O^{p}\left(\operatorname{Aut}_{\mathcal{F}}\left(P \cdot C_{S}(P)\right)\right)$. Thus (3) holds for $P$, since it holds for the $\mathcal{F}$-centric subgroup $P \cdot C_{S}(P)$. Finally, by (1), every subgroup of $S$ is $\mathcal{F}_{1}^{\bullet}$-conjugate to a subgroup which is fully centralized in $\mathcal{F}$, and thus (3) holds for all $P \leq S$.

(c) This point holds, in fact, without assuming that the fusion system $\mathcal{F}$ be saturated. Assume $\Gamma$ is a $p^{\prime}$-group; then $\theta$ is the trivial homomorphism, and so $S_{H}=\theta^{-1}(H)=S$ for all $H$. Fix $H \leq \Gamma$, and let $P$ be any subgroup of $S$. Since

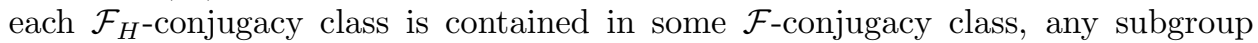
which is fully centralized (fully normalized) in $\mathcal{F}$ is also fully centralized (fully

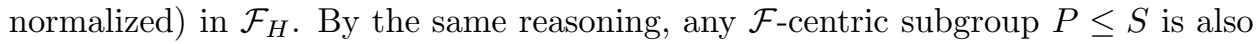
$\mathcal{F}_{H^{-} \text {-centric. }}$

Conversely, assume $P$ is not fully centralized in $\mathcal{F}$, and let $P^{\prime}$ be a subgroup in the $\mathcal{F}$-conjugacy class of $P$ such that $\left|C_{S}\left(P^{\prime}\right)\right|>\left|C_{S}(P)\right|$. Fix some $\varphi \in \operatorname{Iso}_{\mathcal{F}}\left(P, P^{\prime}\right)$. By (1), there are a subgroup $P^{\prime \prime} \leq S$ and isomorphisms $\alpha \in \operatorname{Aut}_{\mathcal{F}}(S)$ and $\varphi^{\prime} \in$ $\operatorname{Iso}_{\mathcal{F}_{1}}\left(P, P^{\prime \prime}\right)$, such that $\alpha^{-1}\left(P^{\prime \prime}\right)=P^{\prime}$ and $\varphi=\left(\left.\alpha\right|_{P^{\prime \prime}}\right) \circ \varphi^{\prime}$. Thus $P^{\prime \prime}$ is $\mathcal{F}_{H^{-}}$ conjugate to $P$, and is $\mathcal{F}$-conjugate to $P^{\prime}$ via a restriction of an $\mathcal{F}$-automorphism of $S$. Hence $\left|C_{S}\left(P^{\prime \prime}\right)\right|=\left|C_{S}\left(P^{\prime}\right)\right|>\left|C_{S}(P)\right|$, and this shows that $P$ is not fully centralized in $\mathcal{F}_{H}$. A similar argument shows that if $P$ is not fully normalized in $\mathcal{F}$ (or not $\mathcal{F}$-centric), then it is not fully normalized in $\mathcal{F}_{H}$ (or not $\mathcal{F}_{H^{-} \text {-centric). }}$

The proof that $\mathcal{F}_{1}^{\bullet} \supseteq O_{*}^{p^{\prime}}(\mathcal{F})$ is identical to the proof of the corresponding result in (b). 
(a) Fix $H \leq \Gamma$. Clearly, $\mathcal{F}_{H}$ is a fusion system over $S_{H}$; we must prove it is saturated. By (b) or (c), each $\mathcal{F}_{H}$-centric subgroup of $S_{H}$ is $\mathcal{F}$-quasicentric. Hence by Theorem 1.5(b), it suffices to prove conditions (I) and (II) in Definition 1.2 for $\mathcal{F}$-quasicentric subgroups $P \leq S_{H}$. Thus we will be working only with subgroups $P \leq S_{H}$ for which $\Theta$ is defined on $\operatorname{Hom}_{\mathcal{F}}(P, S)$.

Proof of (I). Assume $\Gamma$ is a $p^{\prime}$-group; thus $S_{H}=S$. If $P$ is fully normalized in $\mathcal{F}_{H}$, then by (c), $P$ is fully normalized in $\mathcal{F}$, hence it is fully centralized in $\mathcal{F}$ and in $\mathcal{F}_{H}$. Also, $\operatorname{Aut}_{S}(P) \in \operatorname{Syl}_{p}\left(\operatorname{Aut}_{\mathcal{F}}(P)\right)$, and hence $\operatorname{Aut}_{S}(P)$ is also a Sylow $p$-subgroup of $\operatorname{Aut}_{\mathcal{F}_{H}}(P)$. This proves $(\mathrm{I})$ in this case.

Now assume $\Gamma$ is a $p$-group. Fix $P \leq S_{H}$ which is $\mathcal{F}$-quasicentric and fully normalized in $\mathcal{F}_{H}$, and let $\alpha \in \operatorname{Hom}_{\mathcal{F}_{\mathbf{i}}}\left(N_{S}(P), N_{S}\left(P^{\prime}\right)\right)$ be as in (2). In particular, $1 \in \Theta(\alpha)$ by definition of $\mathcal{F}_{1}^{\bullet}$; so by (iv), $\theta\left(\alpha\left(N_{S_{H}}(P)\right)\right)=\theta\left(N_{S_{H}}(P)\right) \leq H$. Hence $\alpha\left(N_{S_{H}}(P)\right) \leq S_{H} \cap N_{S}\left(P^{\prime}\right)=N_{S_{H}}\left(P^{\prime}\right)$, and this is an equality since $P$ is fully normalized in $\mathcal{F}_{H}$. In particular, this shows that $P^{\prime} \leq S_{H}$. The conclusion of (I) holds for $P$ (i.e., $P$ is fully centralized in $\mathcal{F}_{H}$ and $\operatorname{Aut}_{S_{H}}(P) \in \operatorname{Syl}_{p}\left(\operatorname{Aut}_{\mathcal{F}_{H}}(P)\right)$ ) if the conclusion of (I) holds for $P^{\prime}$. So we can assume that $P=P^{\prime}$ is fully normalized in $\mathcal{F}$ and in $\mathcal{F}_{H}$.

Fix $Q \leq S_{H}$ which is $\mathcal{F}_{H}$-conjugate to $P$ and fully centralized in $\mathcal{F}_{H}$, and choose an isomorphism $\psi \in \operatorname{Iso}_{\mathcal{F}_{H}}(P, Q)$. After applying (2) again, we can assume that $Q$ is also fully normalized in $\mathcal{F}$, and hence also fully centralized. Hence $\psi$ extends to some $\psi_{1} \in \operatorname{Hom}_{\mathcal{F}}\left(P \cdot C_{S}(P), Q \cdot C_{S}(Q)\right)$, which is an isomorphism since $P$ and $Q$ are both fully centralized in $\mathcal{F}$. Fix $h \in \Theta\left(\psi^{-1}\right) \cap H$ and $g \in \Theta\left(\psi_{1}\right)$. Then $g h \in \Theta\left(\operatorname{Id}_{Q}\right)=\theta\left(C_{S}(Q)\right)$ by (i) and (ii). Let $a \in C_{S}(Q)$ be such that $\theta(a)=g h$, and set

$$
\psi_{2}=c_{a}^{-1} \circ \psi_{1} \in \operatorname{Iso}_{\mathcal{F}}\left(P \cdot C_{S}(P), Q \cdot C_{S}(Q)\right) .
$$

Thus $\left.\psi_{2}\right|_{P}=\left.\psi_{1}\right|_{P}=\psi$ since $a \in C_{S}(Q)$, and $h^{-1}=\theta(a)^{-1} g \in \Theta\left(\psi_{2}\right)$ by (iii) and (i). By (iv), for all $g \in P \cdot C_{S}(P), \theta\left(\psi_{2}(g)\right)=h^{-1} \theta(g) h$, and hence $\psi_{2}(g) \in H$ if and only if $g \in H$. Thus $\psi_{2}$ sends $C_{S_{H}}(P)$ onto $C_{S_{H}}(Q)$. Since $Q$ is fully centralized in $\mathcal{F}_{H}$, so is $P$.

It remains to show that $\operatorname{Aut}_{S_{H}}(P) \in \operatorname{Syl}_{p}\left(\operatorname{Aut}_{\mathcal{F}_{H}}(P)\right)$. Set $P_{\Theta}=\Theta\left(\operatorname{Id}_{P}\right)=$ $\theta\left(C_{S}(P)\right)$ for short (see (ii)). By (v), $\Theta$ restricts to a homomorphism

$$
\Theta_{P}: \operatorname{Aut}_{\mathcal{F}}(P) \longrightarrow N_{\Gamma}\left(P_{\Theta}\right) / P_{\Theta} .
$$

Set

$$
\widehat{H}=\left\{h P_{\Theta} \mid h \in H \cap N_{\Gamma}\left(P_{\Theta}\right)\right\} \leq N_{\Gamma}\left(P_{\Theta}\right) / P_{\Theta} ;
$$

then $\operatorname{Aut}_{\mathcal{F}_{H}}(P)=\Theta_{P}{ }^{-1}(\widehat{H})$ by definition of $\mathcal{F}_{H}$. For any $\varphi=c_{a} \in \operatorname{Aut}_{S}(P) \cap$ Aut $_{\mathcal{F}_{H}}(P)$ (where $a \in N_{S}(P)$ ), $\Theta(\varphi)=\theta(a) \cdot \theta\left(C_{S}(P)\right.$ ) by (ii) and (iii); and thus $\Theta(\varphi)$ contains some $h \in H$ where $h=\theta(b)$ for some $b \in a \cdot C_{S}(P)$. Hence $b \in S_{H}$, and $\varphi=c_{b} \in \operatorname{Aut}_{S_{H}}(P)$. This shows that

$$
\operatorname{Aut}_{S_{H}}(P)=\operatorname{Aut}_{S}(P) \cap \operatorname{Aut}_{\mathcal{F}_{H}}(P)=\operatorname{Aut}_{S}(P) \cap \Theta_{P}^{-1}(\widehat{H}) .
$$

Also, $\Theta_{P}\left(\operatorname{Aut}_{S}(P)\right)=\Theta_{P}\left(\operatorname{Aut}_{\mathcal{F}}(P)\right)$, since $\operatorname{Aut}_{S}(P) \in \operatorname{Syl}_{p}\left(\operatorname{Aut}_{\mathcal{F}}(P)\right)$ and $\Gamma$ is a $p$-group, and hence

$$
\left[\operatorname{Aut}_{S}(P): \operatorname{Aut}_{S_{H}}(P)\right]=\left[\operatorname{Im}\left(\Theta_{P}\right): \widehat{H}\right]=\left[\operatorname{Aut}_{\mathcal{F}}(P): \operatorname{Aut}_{\mathcal{F}_{H}}(P)\right] .
$$

Since $\operatorname{Aut}_{S}(P) \in \operatorname{Syl}_{P}\left(\operatorname{Aut}_{\mathcal{F}}(P)\right)$ (i.e., $\left[\operatorname{Aut}_{\mathcal{F}}(P): \operatorname{Aut}_{S}(P)\right]$ is prime to $p$ ), this implies that $\operatorname{Aut}_{S_{H}}(P) \in \operatorname{Syl}_{p}\left(\operatorname{Aut}_{\mathcal{F}_{H}}(P)\right)$. 
Proof of (II). Fix a morphism $\varphi \in \operatorname{Iso}_{\mathcal{F}_{H}}(P, Q)$, for some $\mathcal{F}$-quasicentric $P, Q \leq S_{H}$ such that $Q=\varphi(P)$ is fully centralized in $\mathcal{F}_{H}$, and set

$$
N_{\varphi}=\left\{g \in N_{S_{H}}(P) \mid \varphi c_{g} \varphi^{-1} \in \operatorname{Aut}_{S_{H}}(Q)\right\} .
$$

By (2), there is a subgroup $Q^{\prime}$ fully normalized in $\mathcal{F}$, and a morphism $\psi \in$ $\operatorname{Hom}_{\mathcal{F}_{1}}\left(N_{S}(Q), N_{S}\left(Q^{\prime}\right)\right)$, such that $\psi(Q)=Q^{\prime}$. By condition (II) for the saturated fusion system $\mathcal{F}$, there is $\bar{\varphi}_{1} \in \operatorname{Hom}_{\mathcal{F}}\left(N_{\varphi}, N_{S}\left(Q^{\prime}\right)\right)$ such that $\left.\bar{\varphi}_{1}\right|_{P}=\psi \circ \varphi$. Fix some

$$
x \in \Theta\left(\bar{\varphi}_{1}\right) \subseteq \Theta(\psi \varphi)=\Theta(\varphi)
$$

(where the last equality holds since $1 \in \Theta(\psi)$ ). Since $\varphi \in \operatorname{Mor}\left(\mathcal{F}_{H}\right)$, there is $h \in H$ such that $h \in \Theta(\varphi)$, and thus

$$
h x^{-1} \in \Theta\left(\operatorname{Id}_{Q^{\prime}}\right)=\theta\left(C_{S}\left(Q^{\prime}\right)\right) .
$$

Fix $a \in C_{S}\left(Q^{\prime}\right)$ such that $h x^{-1}=\theta(a)$, and set

$$
\bar{\varphi}_{2}=c_{a} \circ \bar{\varphi}_{1} \in \operatorname{Hom}_{\mathcal{F}}\left(N_{\varphi}, N_{S}\left(Q^{\prime}\right)\right) .
$$

Then by (i), $h \in \Theta\left(\bar{\varphi}_{2}\right)$, so $\bar{\varphi}_{2} \in \operatorname{Hom}_{\mathcal{F}_{H}}\left(N_{\varphi}, N_{S_{H}}\left(Q^{\prime}\right)\right)$; and $\left.\bar{\varphi}_{2}\right|_{P}=\left.\bar{\varphi}_{1}\right|_{P}$ since $a \in C_{S}\left(Q^{\prime}\right)$. Since $Q$ is fully centralized in $\mathcal{F}_{H}, \psi$ sends $C_{S_{H}}(Q)$ isomorphically onto $C_{S_{H}}\left(Q^{\prime}\right)$; and hence (by definition of $\left.N_{\varphi}\right) \bar{\varphi}_{2}\left(N_{\varphi}\right) \leq \operatorname{Im}(\psi)$. So $\bar{\varphi} \stackrel{\text { def }}{=} \psi^{-1} \circ \bar{\varphi}_{2}$ sends $N_{\varphi}$ into $N_{S_{H}}(Q)$ and extends $\varphi$.

We next extend Proposition 3.8 to a result about linking systems and $p$-local finite groups. The main point of the following theorem is that for any $p$-local finite group $(S, \mathcal{F}, \mathcal{L})$ and any epimorphism $\pi_{1}(|\mathcal{L}|) \stackrel{\widehat{\theta}}{\longrightarrow} \Gamma$, where $\Gamma$ is a finite $p$-group or $p^{\prime}$-group, there is another $p$-local finite group $\left(S_{H}, \mathcal{F}_{H}, \mathcal{L}_{H}\right)$ for each subgroup $H \leq \Gamma$, such that $\left|\mathcal{L}_{H}\right|$ is homotopy equivalent to the covering space of $|\mathcal{L}|$ whose fundamental group is $\widehat{\theta}^{-1}(H)$.

Recall that for any $p$-local finite group $(S, \mathcal{F}, \mathcal{L})$ with associated quasicentric linking system $\mathcal{L}^{q}, j: S \rightarrow \pi_{1}(|\mathcal{L}|) \cong \pi_{1}\left(\left|\mathcal{L}^{q}\right|\right)$ denotes the homomorphism induced by the distinguished monomorphism $\delta_{S}: S \rightarrow \operatorname{Aut}_{\mathcal{L}}(S)$, and $J: \mathcal{L}^{q} \rightarrow \mathcal{B}\left(\pi_{1}\left(\left|\mathcal{L}^{q}\right|\right)\right)$ is the functor which sends morphisms to loops as defined in Section 1. Let $\widehat{\theta}$ be a homomorphism from $\pi_{1}\left(\left|\mathcal{L}^{q}\right|\right)$ to a group $\Gamma$, and set

$$
\theta=\widehat{\theta} \circ j \in \operatorname{Hom}(S, \Gamma) \quad \text { and } \quad \widehat{\Theta}=\mathcal{B}(\widehat{\theta}) \circ J: \mathcal{L}^{q} \longrightarrow \mathcal{B}(\Gamma) .
$$

Note that these depend on a choice of a compatible set of inclusions $\left\{\iota_{P}^{Q}\right\}$ for $\mathcal{L}^{q}$ (since $J$ depends on such a choice). For any subgroup $H \leq \Gamma$, let $\mathcal{L}_{H}^{\bullet} \subseteq \mathcal{L}^{q}$ be the subcategory with the same objects and with morphism set $\widehat{\Theta}^{-1}(H)$; and let $\mathcal{L}_{H}^{q} \subseteq \mathcal{L}_{H}^{\bullet}$ be the full subcategory obtained by restricting to subgroups of $S_{H} \stackrel{\text { def }}{=}$ $\theta^{-1}(H)$. Finally, let $\mathcal{F}_{H}$ be the fusion system over $S_{H}$ generated by $\pi\left(\mathcal{L}_{H}^{q}\right) \subseteq \mathcal{F}^{q}$ and restrictions of morphisms, and let $\mathcal{L}_{H} \subseteq \mathcal{L}_{H}^{q}$ be the full subcategory on those objects which are $\mathcal{F}_{H}$-centric.

Theorem 3.9. Let $(S, \mathcal{F}, \mathcal{L})$ be a p-local finite group, let $\mathcal{L}^{q}$ be its associated quasicentric linking system, and let $\pi: \mathcal{L}^{q} \rightarrow \mathcal{F}$ be the projection. Assume that a compatible set of inclusions $\left\{\iota_{P}^{Q}\right\}$ has been chosen for $\mathcal{L}^{q}$. Fix a finite group $\Gamma$ which is a p-group or a $p^{\prime}$-group, and a surjective homomorphism

$$
\widehat{\theta}: \pi_{1}\left(\left|\mathcal{L}^{q}\right|\right) \longrightarrow \Gamma \text {. }
$$


Set $\theta=\widehat{\theta} \circ j: S \longrightarrow \Gamma$. Fix $H \leq \Gamma$, and set $S_{H}=\theta^{-1}(H)$. Then $\left(S_{H}, \mathcal{F}_{H}, \mathcal{L}_{H}\right)$ is also a p-local finite group, and (via the inclusion of $\mathcal{L}_{H}$ into $\mathcal{L}^{q}$ ) $\left|\mathcal{L}_{H}\right|$ is homotopy equivalent to the covering space of $\left|\mathcal{L}^{q}\right| \simeq|\mathcal{L}|$ with fundamental group $\widehat{\theta}^{-1}(H)$.

Proof. Define $\Theta: \operatorname{Mor}\left(\mathcal{F}^{q}\right) \rightarrow \mathfrak{S u b}(\Gamma)$ by setting $\Theta(\alpha)=\widehat{\Theta}\left(\pi^{-1}(\alpha)\right)$, where $\widehat{\Theta}=$ $\mathcal{B}(\widehat{\theta}) \circ J$ as above. Then $\Theta$ and $\theta$ satisfy hypotheses (i)-(iv) of Definition 3.6. points (i) and (ii) follow from $(\mathrm{A})_{q}$, while (iii) follows from Proposition 1.13 and (iv) from $(\mathrm{C})_{q}$. Thus $(\Gamma, \theta, \Theta)$ is a fusion mapping triple on $\mathcal{F}^{q}$, and $\mathcal{F}_{H}$ is a saturated fusion system over $S_{H}$ by Proposition 3.8 .

Let $O_{*}^{p}(\mathcal{F})$ and $O_{*}^{p^{\prime}}(\mathcal{F})$ be the categories of Definition 3.3. and let $O_{*}^{p^{\prime}}(\mathcal{F})^{q} \subseteq$ $O_{*}^{p^{\prime}}(\mathcal{F})$ and $O_{*}^{p}(\mathcal{F})^{q} \subseteq O_{*}^{p}(\mathcal{F})$ be the full subcategories whose objects are the $\mathcal{F}$ quasicentric subgroups of $S$. By Proposition $3.8(\mathrm{~b}),(\mathrm{c}), \pi\left(\mathcal{L}_{1}^{\bullet}\right)$ contains $O_{*}^{p^{\prime}}(\mathcal{F})^{q}$ (if $\Gamma$ is a $p^{\prime}$-group) or $O_{*}^{p}(\mathcal{F})^{q}$ (if $\Gamma$ is a $p$-group). Hence in either case, by Lemma 3.4(b), all morphisms in $\mathcal{L}^{q}$ are composites of morphisms in $\mathcal{L}_{1}^{\bullet}$ and restrictions of morphisms in $\operatorname{Aut}_{\mathcal{L}}(S)$. Since $\mathcal{L}_{1}^{\bullet}=\widehat{\Theta}^{-1}(1)$, by definition, and since $\widehat{\Theta}(\alpha)=\widehat{\Theta}(\beta)$ whenever $\alpha$ is a restriction of $\beta$, this shows that $\widehat{\Theta}$ restricts to a surjection of $\operatorname{Aut}_{\mathcal{L}}(S)$ onto $\Gamma$. In particular, this implies that

(1) for all $P \in \operatorname{Ob}\left(\mathcal{L}^{q}\right)$ and for all $g \in \Gamma$,

$$
\exists P^{\prime} \leq S \text { and } \alpha \in \operatorname{Iso}_{\mathcal{L}^{q}}\left(P, P^{\prime}\right) \text { where } \widehat{\Theta}(\alpha)=g ;
$$

and in fact, $\alpha$ can always be chosen to be the restriction of an automorphism of $S$.

We start by proving that $\mathcal{L}_{H}^{q}$ is a linking system associated to $\mathcal{F}_{H}$ (for its set of objects), and hence that $\mathcal{L}_{H}$ is a centric linking system. Let $P \leq S_{H}$ be a $\mathcal{F}$-quasicentric subgroup, and choose $g \in P \cdot C_{S}(P)$. By construction, $\widehat{\Theta}\left(\delta_{S}(g)\right)=$ $\theta(g)$, and $\widehat{\Theta}\left(\iota_{P}\right)=1$. In particular, the inclusion morphisms are in $\mathcal{L}_{H}$. Also, $\iota_{P} \circ \delta_{P}(g)=\delta_{S}(g) \circ \iota_{P}$ by definition of an inclusion morphism (Definition 1.11). Hence $\widehat{\Theta}\left(\delta_{P}(g)\right)=\theta(g)$ in this situation; and in particular $\delta_{P}(g) \in \operatorname{Aut}_{\mathcal{L}_{H}^{q}}(P)$ if and only if $g \in S_{H}$. Thus $\delta_{P}$ restricts to a distinguished monomorphism

$$
P \cdot C_{S_{H}}(P) \longrightarrow \operatorname{Aut}_{\mathcal{L}_{H}^{q}}(P)
$$

for $\mathcal{L}_{H}^{q}$, and axiom $(\mathrm{D})_{q}$ is satisfied. Moreover, if $f, f^{\prime} \in \operatorname{Mor}_{\mathcal{L}_{H}^{q}}(P, Q)$ are such that $\pi(f)=\pi\left(f^{\prime}\right)$ in $\operatorname{Hom}_{\mathcal{F}}(P, Q)$, and $g \in C_{S}(P)$ is the unique element such that $f^{\prime}=f \circ \delta_{P}(g)$ (using axiom $(\mathrm{A})_{q}$ for $\left.\mathcal{L}^{q}\right)$, then $\widehat{\Theta}\left(\delta_{P}(g)\right)=1$, and hence $g \in S_{H}$. This shows that axiom $(\mathrm{A})_{q}$ for $\mathcal{L}_{H}^{q}$ holds. Axioms $(\mathrm{B})_{q}$ and $(\mathrm{C})_{q}$ for $\mathcal{L}_{H}^{q}$ also follow immediately from the same properties for $\mathcal{L}^{q}$.

We have now shown that $\left(S_{H}, \mathcal{F}_{H}, \mathcal{L}_{H}\right)$ is a $p$-local finite group. It remains to show that $\left|\mathcal{L}_{H}\right|$ is homotopy equivalent to a certain covering space of $\left|\mathcal{L}^{q}\right| \simeq|\mathcal{L}|$. We show this by first choosing certain full subcategories $\mathcal{L}^{x} \subseteq \mathcal{L}^{q}$ and $\mathcal{L}_{H}^{x} \subseteq \mathcal{L}_{H}^{q}$ such that $\left|\mathcal{L}^{x}\right| \simeq\left|\mathcal{L}^{q}\right| \simeq|\mathcal{L}|$ and $\left|\mathcal{L}_{H}^{x}\right| \simeq\left|\mathcal{L}_{H}^{q}\right| \simeq\left|\mathcal{L}_{H}\right|$, and then proving directly that $\left|\mathcal{L}_{H}^{x}\right|$ is a covering space of $\left|\mathcal{L}^{x}\right|$.

If $\Gamma$ is a $p^{\prime}$-group, then for any $P \leq S_{H}=S, P$ is $\mathcal{F}_{H}$-centric if and only if it is $\mathcal{F}$-centric (Proposition 3.8(c)). By the above remarks, $\mathcal{L}_{H}$ is a centric linking system associated to $\mathcal{F}_{H}$. Set $\mathcal{L}^{x}=\mathcal{L}$ and $\mathcal{L}_{H}^{x}=\mathcal{L}_{H}$ in this case.

If $\Gamma$ is a $p$-group, then for any $P \leq S_{H}, P$ is $\mathcal{F}_{H}$-quasicentric if and only if it is $\mathcal{F}$-quasicentric (Proposition $3.8(b)$ ). So by what was just shown, $\mathcal{L}_{H}^{q}$ is a quasicentric linking system associated to $\mathcal{F}_{H}$ which extends $\mathcal{L}_{H}$. Let $\mathcal{L}^{x} \subseteq \mathcal{L}^{q}$ be the full subcategory whose objects are those subgroups $P \leq S$ such that $P \cap S_{H}$ is 
$\mathcal{F}$-quasicentric. Set $S_{1}=\operatorname{Ker}(\theta)$, and let $\mathcal{F}_{1} \subseteq \mathcal{F}$ be the saturated fusion system over $S_{1}$ defined in Proposition 3.8. The definition of $\theta$ as a restriction of $\widehat{\Theta}$ ensures that $\theta(g)$ and $\theta\left(g^{\prime}\right)$ are $\Gamma$-conjugate whenever $g, g^{\prime}$ are $\mathcal{F}$-conjugate; in particular, no element of $S_{1}$ is $\mathcal{F}$-conjugate to any element of $S \backslash S_{1}$. Hence by Lemma 3.5. for each $P \leq S$ which is $\mathcal{F}$-centric and $\mathcal{F}$-radical, $P \cap S_{1}$ is $\mathcal{F}_{1}$-centric, hence $\mathcal{F}$ quasicentric, so $P \cap S_{H}$ is $\mathcal{F}$-quasicentric, and thus $P \in \operatorname{Ob}\left(\mathcal{L}^{x}\right)$. So the inclusion of $\left|\mathcal{L}^{x}\right|$ in $\left|\mathcal{L}^{q}\right|$ is a homotopy equivalence by Proposition 1.12.

Still assuming $\Gamma$ is a $p$-group, let $\mathcal{L}_{H}^{x} \subseteq \mathcal{L}^{x}$ be the subcategory with the same objects, where $\operatorname{Mor}\left(\mathcal{L}_{H}^{x}\right)=\widehat{\Theta}^{-1}(H)$. For each $P \in \operatorname{Ob}\left(\mathcal{L}_{H}^{x}\right)=\operatorname{Ob}\left(\mathcal{L}^{x}\right), P \cap S_{H}$ is $\mathcal{F}$ quasicentric by assumption, hence $\mathcal{F}_{H}$-quasicentric; and by Proposition 1.13, each $\varphi \in \operatorname{Mor}_{\mathcal{L}_{H}^{x}}(P, Q)$ restricts to a unique morphism $\varphi_{H} \in \operatorname{Mor}_{\mathcal{L}_{H}^{x}}\left(P \cap S_{H}, Q \cap S_{H}\right)$. These restrictions define a deformation retraction from $\left|\mathcal{L}_{H}^{x}\right|$ to $\left|\mathcal{L}_{H}^{q}\right|$, and thus the inclusion of categories induces a homotopy equivalence $\left|\mathcal{L}_{H}^{x}\right| \simeq\left|\mathcal{L}_{H}^{q}\right| \simeq\left|\mathcal{L}_{H}\right|$.

Thus in both cases, we have chosen categories $\mathcal{L}_{H}^{x} \subseteq \mathcal{L}^{x}$ with the same objects, where $\mathcal{L}^{x}$ is a full subcategory of $\mathcal{L}^{q}$ and $\operatorname{Mor}\left(\mathcal{L}_{H}^{x}\right)=\operatorname{Mor}\left(\mathcal{L}^{x}\right) \cap \widehat{\Theta}^{-1}(H)$, and where $\left|\mathcal{L}^{x}\right| \simeq|\mathcal{L}|$ and $\left|\mathcal{L}_{H}^{x}\right| \simeq\left|\mathcal{L}_{H}\right|$. Let $\mathcal{E}_{\Gamma}(\Gamma / H)$ be the category with object set $\Gamma / H$, and with a morphism $g$ from $a H$ to $g a H$ for each $g \in \Gamma$ and $a H \in \Gamma / H$. Thus $\operatorname{Aut}_{\mathcal{E}_{\Gamma}(\Gamma / H)}(1 \cdot H) \cong H$, and $\left|\mathcal{E}_{\Gamma}(\Gamma / H)\right|=E G / H \simeq B H$.

Let $\widetilde{\mathcal{L}}$ be the pullback category in the following square:

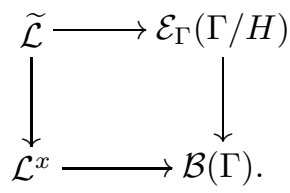

Thus $\operatorname{Ob}(\widetilde{\mathcal{L}})=\operatorname{Ob}\left(\mathcal{L}^{x}\right) \times \Gamma / H$, and $\operatorname{Mor}(\widetilde{\mathcal{L}})$ is the set of pairs of morphisms in $\mathcal{L}^{x}$ and $\mathcal{E}_{\Gamma}(\Gamma / H)$ which get sent to the same morphism in $\mathcal{B}(\Gamma)$. Then $\mathcal{L}_{H}^{x}$ can be identified with the full subcategory of $\widetilde{\mathcal{L}}$ with objects the pairs $(P, 1 \cdot H)$ for $P \in \operatorname{Ob}\left(\mathcal{L}^{x}\right)$. By $(1)$, each object in $\widetilde{\mathcal{L}}$ is isomorphic to an object in $\mathcal{L}_{H}^{x}$, and so $\left|\mathcal{L}_{H}^{x}\right| \simeq|\widetilde{\mathcal{L}}|$. By construction, $|\widetilde{\mathcal{L}}|$ is the covering space over $\left|\mathcal{L}^{x}\right|$ with fundamental group $\theta^{-1}(H)$. Since $|\widetilde{\mathcal{L}}| \simeq\left|\mathcal{L}_{H}\right|$ and $\left|\mathcal{L}^{x}\right| \simeq|\mathcal{L}|$, this finishes the proof of the last statement.

The following is an immediate corollary to Theorem 3.9

Corollary 3.10. For any $p$-local finite group $(S, \mathcal{F}, \mathcal{L})$, any finite $p$-solvable group $\Gamma$, and any homomorphism

$$
\widehat{\theta}: \pi_{1}(|\mathcal{L}|) \longrightarrow \Gamma,
$$

there is a p-local finite group $\left(S_{0}, \mathcal{F}_{0}, \mathcal{L}_{0}\right)$ such that $\left|\mathcal{L}_{0}\right|$ is homotopy equivalent to the covering space of $|\mathcal{L}|$ with fundamental group $\operatorname{Ker}(\widehat{\theta})$. Furthermore, this can be chosen such that $S_{0}$ is a subgroup of $S$ and $\mathcal{F}_{0}$ is a subcategory of $\mathcal{F}$.

For any $p$-local finite group $(S, \mathcal{F}, \mathcal{L})$, since $\mathcal{F}$ is a finite category, Corollary 3.10 implies that there is a unique maximal $p$-solvable quotient group of $\pi_{1}(|\mathcal{L}|)$, which is finite. In contrast, if we look at arbitrary finite quotient groups of the fundamental group, they can be arbitrarily large.

As one example, consider the case where $p=2, S \in \operatorname{Syl}_{2}\left(A_{6}\right)$ (so $S \cong D_{8}$ ), $\mathcal{F}=\mathcal{F}_{S}\left(A_{6}\right)$, and $\mathcal{L}=\mathcal{L}_{S}^{c}\left(A_{6}\right)$. It is not hard to show directly, using Van Kampen's 
theorem, that $\pi_{1}(|\mathcal{L}|) \cong \Sigma_{4} * \Sigma_{4}$ : the amalgamated free product of two copies of $\Sigma_{4}$ intersecting in $S$, where each of the two subgroups $C_{2}^{2}$ in $D_{8}$ is normalized by one of the $\Sigma_{4}$. Thus $\pi_{1}(|\mathcal{L}|)$ surjects onto any finite group $\Gamma$ which is generated by two copies of $\Sigma_{4}$ intersecting in the same way. This is the case when $\Gamma=A_{6}$, and also when $\Gamma=P S L_{2}(q)$ for any $q \equiv \pm 9(\bmod 16)$. However, the kernel of any such homomorphism defined on $\pi_{1}(|\mathcal{L}|)$ is torsion free (and infinite), and hence cannot be the fundamental group of the geometric realization of any centric linking system. In fact, in this case, there is no nontrivial homomorphism from $\pi_{1}(|\mathcal{L}|)$ to a finite 2-solvable group.

\section{Fusion subsystems And EXTEnsions of $p$-POWER IndeX}

Recall that for any saturated fusion system $\mathcal{F}$ over a $p$-group $S$, we defined $O_{\mathcal{F}}^{p}(S) \triangleleft S$ to be the subgroup generated by all elements of the form $g^{-1} \alpha(g)$, for $g \in P \leq S$, and $\alpha \in \operatorname{Aut}_{\mathcal{F}}(P)$ of order prime to $p$. In this section, we classify all saturated fusion subsystems of $p$-power index in a given saturated fusion system $\mathcal{F}$ over $S$, and show that there is a one-to-one correspondence between such subsystems and the subgroups of $S$ which contain $O_{\mathcal{F}}^{p}(S)$. In particular, there is a unique minimal subsystem $O^{p}(\mathcal{F})$ of this type, which is a fusion system over $O_{\mathcal{F}}^{p}(S)$. This result has been shown independently by Puig $\mathrm{Pu} 3$, when $\mathcal{F}$ is assumed to have an associated linking system. We then look at extensions, and describe the procedure for finding all larger saturated fusion systems of which $\mathcal{F}$ is a fusion subsystem of $p$-power index.

4.1. Subsystems of $p$-power index. In this subsection, we classify all saturated fusion subsystems of $p$-power index in a given saturated fusion system $\mathcal{F}$, and show that there is a unique minimal subsystem $O^{p}(\mathcal{F})$ of this type. We also show that there is a bijective correspondence between subgroups $T$ of the finite $p$-group

$$
\Gamma_{p}(\mathcal{F}) \stackrel{\text { def }}{=} S / O_{\mathcal{F}}^{p}(S)
$$

and fusion subsystems $\mathcal{F}_{T}$ of $p$-power index in $\mathcal{F}$. We have already seen that $\Gamma_{p}(\mathcal{F})=S / O_{\mathcal{F}}^{p}(S)$ is isomorphic to $\pi_{1}\left(|\mathcal{L}|_{p}^{\wedge}\right)$ for any centric linking system $\mathcal{L}$ associated to $\mathcal{F}$, and our result also shows that all (connected) covering spaces of $|\mathcal{L}|_{p}^{\wedge}$ are realized as classifying spaces of subsystems of $p$-power index. The index of $\mathcal{F}_{T}$ in $\mathcal{F}$ can then be defined to be the index of $T$ in $\Gamma_{p}(\mathcal{F})$, or equivalently the covering degree of the covering space.

The main step in doing this is to construct a fusion mapping triple $\left(\Gamma_{p}(\mathcal{F}), \theta, \Theta\right)$ for $\mathcal{F}^{q}$, where $\theta$ is the canonical surjection of $S$ onto $\Gamma_{p}(\mathcal{F})$. This construction parallels very closely the construction in Proposition 2.4 of a functor from $\mathcal{L}^{q}$ to $\mathcal{B}\left(\Gamma_{p}(\mathcal{F})\right)$, when $\mathcal{L}$ is a linking system associated to $\mathcal{F}$. In fact, we could in principal state and prove the two results simultaneously, but the extra terminology which that would require seemed to add more complications than would be saved by combining the two.

The following lemma provides a very general, inductive tool for constructing explicit fusion mapping triples.

Lemma 4.1. Fix a saturated fusion system $\mathcal{F}$ over a p-group $S$. Let $\mathcal{H}_{0}$ be a set of $\mathcal{F}$-quasicentric subgroups of $S$ which is closed under $\mathcal{F}$-conjugacy and overgroups. Let $\mathcal{P}$ be an $\mathcal{F}$-conjugacy class of $\mathcal{F}$-quasicentric subgroups maximal among those 
not in $\mathcal{H}_{0}$, set $\mathcal{H}=\mathcal{H}_{0} \cup \mathcal{P}$, and let $\mathcal{F}^{\mathcal{H}_{0}} \subseteq \mathcal{F}^{\mathcal{H}} \subseteq \mathcal{F}^{q}$ be the full subcategories with these objects. Fix a group $\Gamma$ and a homomorphism $\theta: S \rightarrow \Gamma$, and let

$$
\Theta_{0}: \operatorname{Mor}\left(\mathcal{F}^{\mathcal{H}_{0}}\right) \longrightarrow \mathfrak{S} \mathfrak{u} \mathfrak{b}(\Gamma)
$$

be such that $\left(\Gamma, \theta, \Theta_{0}\right)$ is a fusion mapping triple for $\mathcal{F}^{\mathcal{H}_{0}}$. Let $P \in \mathcal{P}$ be fully normalized in $\mathcal{F}$, and fix a homomorphism

$$
\Theta_{P}: \operatorname{Aut}_{\mathcal{F}}(P) \longrightarrow N_{\Gamma}\left(\theta\left(C_{S}(P)\right)\right) / \theta\left(C_{S}(P)\right)
$$

such that the following two conditions hold:

$(+) x \theta(g) x^{-1}=\theta(\alpha(g))$ for all $g \in P, \alpha \in \operatorname{Aut}_{\mathcal{F}}(P)$, and $x \in \Theta_{P}(\alpha)$.

(*) For all $P \supsetneqq Q \leq S$ such that $P \triangleleft Q$ and $Q$ is fully normalized in $N_{\mathcal{F}}(P)$, and for all $\alpha \in \operatorname{Aut}_{\mathcal{F}}(P)$ and $\beta \in \operatorname{Aut}_{\mathcal{F}}(Q)$ such that $\alpha=\left.\beta\right|_{P}, \Theta_{P}(\alpha) \supseteq$ $\Theta_{0}(\beta)$.

Then there is a unique extension of $\Theta_{0}$ to a fusion mapping triple $(\Gamma, \theta, \Theta)$ for $\mathcal{F}^{\mathcal{H}}$ such that $\Theta(\alpha)=\Theta_{P}(\alpha)$ for all $\alpha \in \operatorname{Aut}_{\mathcal{F}}(P)$.

Proof. Note that $(+)$ is just point (v) of Lemma 3.7 applied to the subgroup $P$, while $(*)$ is just point (vi) applied to restrictions to $P$. So both of these conditions are necessary if we want to be able to extend $\Theta_{0}$ and $\Theta_{P}$ to a fusion mapping triple for $\mathcal{F}^{\mathcal{H}}$.

The uniqueness of the extension is an immediate consequence of Alperin's fusion theorem, in the form of Theorem 1.5(a). The proof of existence is almost identical to the proof of Lemma 2.3. so we just sketch it here briefly.

We first show that we can replace $(*)$ by the following (a priori stronger) statement:

(**) for all $Q, Q^{\prime} \leq S$ which strictly contain $P$, and for all $\beta \in \operatorname{Hom}_{\mathcal{F}}\left(Q, Q^{\prime}\right)$ and $\alpha \in \operatorname{Aut}_{\mathcal{F}}(P)$ such that $\alpha=\left.\beta\right|_{P}, \Theta_{P}(\alpha) \supseteq \Theta_{0}(\beta)$.

It suffices to show this when $P$ is normal in $Q$ and $Q^{\prime}$, since otherwise we can replace $Q$ and $Q^{\prime}$ by $N_{Q}(P)$ and $N_{Q^{\prime}}(P)$. In this case, $\beta \in \operatorname{Hom}_{N_{\mathcal{F}}(P)}\left(Q, Q^{\prime}\right)$, and by Theorem 1.5(a) (Alperin's fusion theorem), it is a composite of restrictions of automorphisms of subgroups fully normalized in $N_{\mathcal{F}}(P)$. So it suffices to prove (**) when $\beta$ is such an automorphism, and this is what is assumed in $(*)$.

Now fix any morphism $\varphi \in \operatorname{Hom}_{\mathcal{F}}\left(P_{1}, Q\right)$ which lies in $\mathcal{F}^{\mathcal{H}}$ but not in $\mathcal{F}^{\mathcal{H}_{0}}$; thus $P_{1} \in \mathcal{P}$. Set $P_{2}=\varphi\left(P_{1}\right) \leq Q$, and let $\varphi^{\prime} \in \operatorname{Iso}_{\mathcal{F}}\left(P_{1}, P_{2}\right)$ be the "restriction" of $\varphi$. By Lemma 1.3, there are isomorphisms $\bar{\varphi}_{i} \in \operatorname{Iso}_{\mathcal{F}^{q}}\left(N_{S}\left(P_{i}\right), N_{i}\right)$, for some $N_{i} \leq N_{S}(P)$ containing $P$, which restrict to isomorphisms $\varphi_{i} \in \operatorname{IsO}_{\mathcal{F}^{q}}\left(P_{i}, P\right)$. Fix elements $x_{i} \in \Theta_{0}\left(\bar{\varphi}_{i}\right)$. Set $\psi=\varphi_{2} \circ \varphi^{\prime} \circ \varphi_{1}^{-1} \in \operatorname{Aut}_{\mathcal{F}^{q}}(P)$. Thus $\varphi^{\prime}=\varphi_{2}^{-1} \circ \psi \circ \varphi_{1}$, and we define

$$
\Theta(\varphi)=\Theta\left(\varphi^{\prime}\right)=x_{2}^{-1} \cdot \Theta_{P}(\psi) \cdot x_{1} .
$$

This is independent of the choice of $x_{i}$, since $\Theta_{0}\left(\bar{\varphi}_{i}\right) \subseteq \theta\left(C_{S}(P)\right) \cdot x_{i}$ by axioms (i) and (ii) in the definition of a fusion mapping triple. It is independent of the choice of $\varphi_{1}$ and $\varphi_{2}$ by the same argument as was used in the proof of Lemma 2.3 (and this is where we need point $(* *))$. Conditions (i)-(iv) are easily checked. For example, (iv) - the condition that $x \theta(g) x^{-1}=\theta(\alpha(g))$ whenever $g \in P_{1}, \varphi \in \operatorname{Hom}_{\mathcal{F}}\left(P_{1}, P_{2}\right)$, and $x \in \Theta(\varphi)$ - holds when $\varphi$ can be extended to a larger subgroup since $\left(\Gamma, \theta, \Theta_{0}\right)$ is already a fusion mapping triple, holds for $\varphi \in \operatorname{Aut}_{\mathcal{F}}(P)$ by $(+)$, and thus holds in the general case since $\Theta(\varphi)$ was defined via a composition of such morphisms. Thus $(\Gamma, \theta, \Theta)$ is a fusion mapping triple on $\mathcal{F}^{\mathcal{H}}$. 
The construction of a fusion mapping triple to $\Gamma_{p}(\mathcal{F})$ in the following lemma is a first application of Lemma 4.1. Another application will be given in the next section.

Lemma 4.2. Let $\mathcal{F}$ be a saturated fusion system over a p-group $S$, and let

$$
\theta: S \longrightarrow \Gamma_{p}(\mathcal{F})=S / O_{\mathcal{F}}^{p}(S)
$$

be the projection. Then there is a fusion mapping triple $\left(\Gamma_{p}(\mathcal{F}), \theta, \Theta\right)$ on $\mathcal{F}^{q}$.

Proof. The function $\Theta$ will be constructed inductively, using Lemma 4.1. Let $\mathcal{H}_{0} \subseteq \mathrm{Ob}\left(\mathcal{F}^{q}\right)$ be a subset (possibly empty) which is closed under $\mathcal{F}$-conjugacy and overgroups. Let $\mathcal{P}$ be an $\mathcal{F}$-conjugacy class of $\mathcal{F}$-quasicentric subgroups maximal among those not in $\mathcal{H}_{0}$, set $\mathcal{H}=\mathcal{H}_{0} \cup \mathcal{P}$, and let $\mathcal{F}^{\mathcal{H}_{0}} \subseteq \mathcal{F}^{\mathcal{H}} \subseteq \mathcal{F}^{q}$ be the full subcategories with these objects. Assume we have already constructed a fusion mapping triple $\left(\Gamma_{p}(\mathcal{F}), \theta, \Theta_{0}\right)$ for $\mathcal{F}^{\mathcal{H}_{0}}$.

We recall the notation of Lemma 2.2. If $G$ is any finite group, and $S \in \operatorname{Syl}_{p}(G)$, then

$$
\left.O_{G}^{p}(S) \stackrel{\text { def }}{=}\langle[g, x]| g \in P \leq S, x \in N_{G}(P) \text { of order prime to } p\right\rangle .
$$

By Lemma 2.2, $O_{G}^{p}(S)=S \cap O^{p}(G)$, and hence $G / O^{p}(G) \cong S / O_{G}^{p}(S)$.

Fix $P \in \mathcal{P}$ which is fully normalized in $\mathcal{F}$. Let $N_{0}$ be the subgroup generated by commutators $[g, x]$ for $g \in N_{S}(P)$ and $x \in N_{\text {Aut }_{\mathcal{F}}(P)}\left(N_{S}(P)\right)$ of order prime to p. Then $\operatorname{Aut}_{S}(P) \in \operatorname{Syl}_{p}\left(\operatorname{Aut}_{\mathcal{F}}(P)\right)$ and $\operatorname{Aut}_{N_{0}}(P)=O_{\operatorname{Aut}_{\mathcal{F}}(P)}^{p}\left(\operatorname{Aut}_{S}(P)\right)$, and by Lemma 2.2

$$
\left.\operatorname{Aut}_{\mathcal{F}}(P) / O^{p} \operatorname{Aut}_{\mathcal{F}}(P)\right) \cong \operatorname{Aut}_{S}(P) / A u t_{N_{0}}(P) \cong N_{S}(P) /\left\langle N_{0}, C_{S}(P)\right\rangle .
$$

Also, $N_{0} \leq O_{\mathcal{F}}^{p}(S)$, and so the inclusion of $N_{S}(P)$ into $S$ induces a homomorphism $\Theta_{P}: \operatorname{Aut}_{\mathcal{F}}(P) \longrightarrow \operatorname{Aut}_{\mathcal{F}}(P) / O^{p}\left(\operatorname{Aut}_{\mathcal{F}}(P)\right)$
$\cong N_{S}(P) /\left\langle N_{0}, C_{S}(P)\right\rangle$$\underset{N_{\Gamma_{p}(\mathcal{F})}\left(\theta\left(C_{S}(P)\right)\right) / \theta\left(C_{S}(P)\right) .}{\cong N_{S}\left(C_{S}(P) \cdot S_{0}\right) /\left(C_{S}(P) \cdot S_{0}\right)}$

Here, we write $S_{0}=O_{\mathcal{F}}^{p}(S)$ for short; thus $\Gamma_{p}(\mathcal{F})=S / S_{0}$ and $\theta\left(C_{S}(P)\right)=$ $C_{S}(P) \cdot S_{0} / S_{0}$.

Point (+) in Lemma 4.1 holds by the construction of $\Theta_{P}$. So it remains only to prove that condition $(*)$ in Lemma 4.1 holds.

To see this, fix $P \supsetneqq Q \leq S$ such that $P \triangleleft Q$ and $Q$ is fully normalized in $N_{\mathcal{F}}(P)$, and fix $\alpha \in \operatorname{Aut}_{\mathcal{F}^{q}}(P)$ and $\beta \in \operatorname{Aut}_{\mathcal{F}^{q}}(Q)$ such that $\alpha=\left.\beta\right|_{P}$. We must show that $\Theta_{P}(\alpha) \supseteq \Theta_{0}(\beta)$. Upon replacing $\alpha$ by $\alpha^{k}$ and $\beta$ by $\beta^{k}$ for some appropriate $k \equiv 1$ $(\bmod p)$, we can assume that both automorphisms have order a power of $p$. Since $Q$ is fully normalized, $\operatorname{Aut}_{N_{S}(P)}(Q)$ is a Sylow subgroup of $\operatorname{Aut}_{N_{\mathcal{F}}(P)}(Q)$; and hence there are automorphisms $\bar{\gamma} \in \operatorname{Aut}_{\mathcal{F}}(Q)$ and $\gamma \in \operatorname{Aut}_{\mathcal{F}}(P)$ of order prime to $p$ such that $\gamma=\left.\bar{\gamma}\right|_{P}$ and $\bar{\gamma} \beta \bar{\gamma}^{-1}=\left.c_{g}\right|_{Q}$ for some $g \in N_{S}(Q) \cap N_{S}(P)$. Then $\gamma \alpha \gamma^{-1}=\left.c_{g}\right|_{P}$. Also, $1 \in \Theta_{P}(\gamma)$ and $1 \in \Theta_{0}(\bar{\gamma})$, since both automorphisms have order prime to $p$. So

$$
\Theta_{0}(\beta)=\Theta\left(\left.c_{g}\right|_{Q}\right)=g \cdot \theta\left(C_{S}(Q)\right) \subseteq g \cdot \theta\left(C_{S}(P)\right)=\Theta_{P}\left(\left.c_{g}\right|_{P}\right)=\Theta_{P}(\alpha) .
$$

Thus, by Lemma 2.3, we can extend $\Theta_{0}$ to a fusion mapping triple on $\mathcal{F}^{\mathcal{H}}$. Upon continuing this procedure, we obtain a fusion mapping triple defined on all of $\mathcal{F}^{q}$.

We now apply Lemma 4.2 to classify fusion subsystems of $p$-power index. Recall that by definition, if $\mathcal{F}$ is a saturated fusion system over $S$ and $\mathcal{F}_{0} \subseteq \mathcal{F}$ is a fusion subsystem over $S_{0} \leq S$, then $\mathcal{F}$ has $p$-power index if and only if $S_{0} \geq O_{\mathcal{F}}^{p}(S)$, and $\operatorname{Aut}_{\mathcal{F}_{0}}(P) \geq O^{p}\left(\operatorname{Aut}_{\mathcal{F}}(P)\right)$ for all $P \leq S_{0}$. 
Theorem 4.3. Fix a saturated fusion system $\mathcal{F}$ over a p-group $S$. Then for each subgroup $T \leq S$ containing $O_{\mathcal{F}}^{p}(S)$, there is a unique saturated fusion system $\mathcal{F}_{T} \subseteq$ $\mathcal{F}$ over $T$ with p-power index. For each such $T, \mathcal{F}_{T}$ has the properties:

(a) a subgroup $P \leq T$ is $\mathcal{F}_{T}$-quasicentric if and only if it is $\mathcal{F}$-quasicentric; and

(b) for each pair $P, Q \leq T$ of $\mathcal{F}$-quasicentric subgroups,

$$
\operatorname{Hom}_{\mathcal{F}_{T}}(P, Q)=\left\{\varphi \in \operatorname{Hom}_{\mathcal{F}}(P, Q) \mid \Theta(\varphi) \cap\left(T / O_{\mathcal{F}}^{p}(S)\right) \neq \emptyset\right\} .
$$

Here,

$$
\Theta: \operatorname{Mor}\left(\mathcal{F}^{q}\right) \longrightarrow \mathfrak{S u b}\left(\Gamma_{p}(\mathcal{F})\right)=\mathfrak{S u b}\left(S / O_{\mathcal{F}}^{p}(S)\right)
$$

is the map of Lemma 4.2 ,

Proof. Let $\mathcal{F}_{T} \subseteq \mathcal{F}$ be the fusion system over $T$ defined on $\mathcal{F}$-quasicentric subgroups by the formula in (b), and then extended to arbitrary subgroups by taking restrictions and composites. (This is the fusion system denoted $\mathcal{F}_{T / O_{\mathcal{F}}^{p}(S)}$ in Proposition 3.8, but we simplify the notation here.) By Proposition 3.8(a), (b) (applied with $\Gamma=\Gamma_{p}(\mathcal{F})$ and $\left.H=T / O_{\mathcal{F}}^{p}(S)\right), \mathcal{F}_{T}$ is saturated, a subgroup $P \leq T$ is $\mathcal{F}_{T^{-}}$

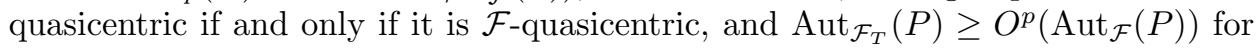
all $P \leq T$.

Now let $\mathcal{F}_{T}^{\prime} \subseteq \mathcal{F}$ be another saturated subsystem over the same subgroup $T$ which also has $p$-power index. We claim that $\mathcal{F}_{T}^{\prime}=\mathcal{F}_{T}$, and thus that $\mathcal{F}_{T}$ is the unique subsystem with these properties. By assumption, for each $P \leq T$, $\operatorname{Aut}_{\mathcal{F}_{T}}(P)$ and $\operatorname{Aut}_{\mathcal{F}_{T}^{\prime}}(P)$ both contain $O^{p}\left(\operatorname{Aut}_{\mathcal{F}}(P)\right)$, and hence each is generated by $O^{p}\left(\operatorname{Aut}_{\mathcal{F}}(P)\right)$ and any one of its Sylow $p$-subgroups. So if $P$ is fully normalized in both $\mathcal{F}_{T}$ and $\mathcal{F}_{T}^{\prime}$, then

$$
\operatorname{Aut}_{\mathcal{F}_{T}}(P)=\left\langle O^{p}\left(\operatorname{Aut}_{\mathcal{F}}(P)\right), \operatorname{Aut}_{T}(P)\right\rangle=\operatorname{Aut}_{\mathcal{F}_{T}^{\prime}}(P) .
$$

In particular, $\operatorname{Aut}_{\mathcal{F}_{T}}(T)=\operatorname{Aut}_{\mathcal{F}_{T}^{\prime}}(T)$. Set $p^{k}=|T|$, fix $0 \leq m<k$, and assume inductively that $\operatorname{Hom}_{\mathcal{F}_{T}}(P, Q)=\operatorname{Hom}_{\mathcal{F}_{T}^{\prime}}(P, Q)$ for all $P, Q \leq T$ of order $>p^{m}$. By Alperin's fusion theorem for saturated fusion systems (Theorem[1.5(a)), if $|P|=p^{m}$, $|Q| \geq p^{m}$, and $P \neq Q$, then all morphisms in $\operatorname{Hom}_{\mathcal{F}_{T}}(P, Q)$ and $\operatorname{Hom}_{\mathcal{F}_{T}^{\prime}}(P, Q)$ are composites of restrictions of morphisms between subgroups of order $>p^{m}$, and hence $\operatorname{Hom}_{\mathcal{F}_{T}}(P, Q)=\operatorname{Hom}_{\mathcal{F}_{T}^{\prime}}(P, Q)$ by the induction hypothesis. In particular, two subgroups of order $p^{m}$ are $\mathcal{F}_{T}$-conjugate if and only if they are $\mathcal{F}_{T}^{\prime}$-conjugate. So for any $P \leq T$ of order $p^{m}, P$ is fully normalized in $\mathcal{F}_{T}$ if and only if it is fully normalized in $\mathcal{F}_{T}^{\prime}$. In either case, $\operatorname{Aut}_{\mathcal{F}_{T}}(P)=\operatorname{Aut}_{\mathcal{F}_{T}^{\prime}}(P)$ : by (1) if $P$ is fully normalized, and by Alperin's fusion theorem again (and the induction hypothesis) if it is not.

We can now define $O^{p}(\mathcal{F})$ as the minimal fusion subsystem of $\mathcal{F}$ of $p$-power index: the unique fusion subsystem over $O_{\mathcal{F}}^{p}(S)$ of $p$-power index. The next theorem will show that when $\mathcal{F}$ has an associated linking system $\mathcal{L}$, then $O^{p}(\mathcal{F})$ has an associated linking system $O^{p}(\mathcal{L})$, and that $\left|O^{p}(\mathcal{L})\right|_{p}^{\wedge}$ is the universal cover of $|\mathcal{L}|_{p}^{\wedge}$.

Theorem 4.4. Fix a p-local finite group $(S, \mathcal{F}, \mathcal{L})$. Then for each subgroup $T \leq S$ containing $O_{\mathcal{F}}^{p}(S)$, there is a unique p-local finite subgroup $\left(T, \mathcal{F}_{T}, \mathcal{L}_{T}\right)$ such that $\mathcal{F}_{T}$ has p-power index in $\mathcal{F}$, and such that $\mathcal{L}_{T}^{q}=\pi^{-1}\left(\mathcal{F}_{T}^{q}\right)$ where $\pi$ is the usual projection of $\mathcal{L}^{q}$ onto $\mathcal{F}^{q}$. Furthermore, $\left|\mathcal{L}_{T}\right|$ is homotopy equivalent, via the inclusion of $\left|\mathcal{L}_{T}^{q}\right| \simeq\left|\mathcal{L}_{T}\right|$ into $\left|\mathcal{L}^{q}\right| \simeq|\mathcal{L}|$, to a covering space of $|\mathcal{L}|$ of degree $[S: T]$. Hence the classifying space $\left|\mathcal{L}_{T}\right|_{p}^{\wedge}$ of $\left(T, \mathcal{F}_{T}, \mathcal{L}_{T}\right)$ is homotopy equivalent to the covering space of $|\mathcal{L}|_{p}^{\wedge}$ with fundamental group $T / O_{\mathcal{F}}^{p}(S)$. 
Proof. Assume a compatible set of inclusions $\left\{\iota_{P}^{Q}\right\}$ has been chosen for $\mathcal{L}^{q}$. By Proposition 2.4 there is a functor $\lambda: \mathcal{L}^{q} \rightarrow \mathcal{B}\left(\Gamma_{p}(\mathcal{F})\right)$ which sends inclusions to the identity, and such that $\lambda\left(\delta_{S}(g)\right)=g$ for all $g \in S$. Hence by Theorem 3.9. $\left(T, \mathcal{F}_{T}, \mathcal{L}_{T}\right)$ is a $p$-local finite group, and $\left|\mathcal{L}_{T}\right|$ is a covering space of $|\mathcal{L}|$. Also, if we write $\mathcal{L}_{1}$ for the linking system over $O_{\mathcal{F}}^{p}(S)$, then the fibration sequences

$$
\left|\mathcal{L}_{1}\right| \rightarrow|\mathcal{L}| \rightarrow B \Gamma_{p}(\mathcal{F}) \quad \text { and } \quad\left|\mathcal{L}_{1}\right| \rightarrow\left|\mathcal{L}_{T}\right| \rightarrow B\left(T / O_{\mathcal{F}}^{p}(S)\right)
$$

are still fibration sequences after $p$-completion [BK, II.5.2(iv)], and hence $\left|\mathcal{L}_{T}\right|_{p}^{\wedge}$ is the covering space of $|\mathcal{L}|_{p}^{\wedge}$ with fundamental group $T / O_{\mathcal{F}}^{p}(S)$. The uniqueness follows from Theorem 4.3 .

Thus there is a bijective correspondence between fusion subsystems of $(S, \mathcal{F})$, or $p$-local finite subgroups of $(S, \mathcal{F}, \mathcal{L})$, of $p$-power index, and subgroups of $S / O_{\mathcal{F}}^{p}(S) \cong$ $\pi_{1}\left(|\mathcal{L}|_{p}^{\wedge}\right)$. The classifying spaces of the $p$-local finite subgroups of $(S, \mathcal{F}, \mathcal{L})$ of $p$ power index are (up to homotopy) just the covering spaces of the classifying space of $(S, \mathcal{F}, \mathcal{L})$.

4.2. Extensions of $p$-power index. We next consider the opposite problem: how to construct extensions of $p$-power index of a given $p$-local finite group. In the course of this construction, we will see that the linking system really is needed to construct an extension of the fusion system. The following definition will be useful.

Definition 4.5. Fix a saturated fusion system $\mathcal{F}$ over a $p$-group $S$. An automorphism $\alpha \in \operatorname{Aut}(S)$ is fusion preserving if it normalizes $\mathcal{F}$; i.e., if it induces an automorphism of the category $\mathcal{F}$ by sending $P$ to $\alpha(P)$ and $\varphi \in \operatorname{Mor}(\mathcal{F})$ to $\alpha \varphi \alpha^{-1} \in \operatorname{Mor}(\mathcal{F})$. Let $\operatorname{Aut}_{\text {fus }}(S, \mathcal{F}) \leq \operatorname{Aut}(S)$ denote the group of all fusion preserving automorphisms, and set

$$
\operatorname{Out}_{\text {fus }}(S, \mathcal{F})=\operatorname{Aut}_{\text {fus }}(S, \mathcal{F}) / \operatorname{Aut}_{\mathcal{F}}(S)
$$

We first describe the algebraic data needed to determine extensions of $p$-power index. Fix a $p$-local finite group $(S, \mathcal{F}, \mathcal{L})$, let $\mathcal{L}^{q}$ be the associated quasicentric linking system, and let $\left\{\iota_{P}^{Q}\right\}$ be a compatible set of inclusions. Then for any $g \in S$, $g$ acts on the set $\operatorname{Mor}\left(\mathcal{L}^{q}\right)$ by composing on the left or right with $\delta_{S}(g)$ and its restrictions. Thus for any $\varphi \in \operatorname{Mor}_{\mathcal{L}^{q}}(P, Q)$, we set

$$
g \varphi=\delta_{Q, g Q g^{-1}}(g) \circ \varphi \in \operatorname{Mor}_{\mathcal{L}^{q}}\left(P, g Q g^{-1}\right)
$$

and

$$
\varphi g=\varphi \circ \delta_{g^{-1} P g, P}(g) \in \operatorname{Mor}_{\mathcal{L}^{q}}\left(g^{-1} P g, Q\right) .
$$

This defines natural left and right actions of $S$ on the set $\operatorname{Mor}\left(\mathcal{L}^{q}\right)$. The resulting conjugation action $\varphi \mapsto g \varphi g^{-1}$ extends to an action on the category, where $g$ sends an object $P$ to $g \mathrm{Pg}^{-1}$. The functor $\pi: \mathcal{L}^{q} \rightarrow \mathcal{F}^{q}$ is equivariant with respect to the conjugation action of $S$ on $\mathcal{L}^{q}$ and the action of $\operatorname{Inn}(S) \leq \operatorname{Aut}_{\text {fus }}(S, \mathcal{F})$ on $\mathcal{F}$.

If $\left(S_{0}, \mathcal{F}_{0}, \mathcal{L}_{0}\right)$ is contained in $(S, \mathcal{F}, \mathcal{L})$ with $p$-power index, and $S_{0} \triangleleft S$, then the $S$ action on $\mathcal{L}$ clearly restricts to an $S$-action on $\mathcal{L}_{0}$. The following theorem provides a converse to this. Given a $p$-local finite group $\left(S_{0}, \mathcal{F}_{0}, \mathcal{L}_{0}\right)$, an extension $S$ of $S_{0}$, and an $S$-action on $\mathcal{L}$ which satisfies certain obvious compatibility conditions, this data always determines a $p$-local finite group which contains $\left(S_{0}, \mathcal{F}_{0}, \mathcal{L}_{0}\right)$ with $p$-power index. 
Theorem 4.6. Fix a p-local finite group $\left(S_{0}, \mathcal{F}_{0}, \mathcal{L}_{0}\right)$, and assume that a compatible set of inclusions $\left\{\iota_{P}^{Q}\right\}$ has been chosen for $\mathcal{L}_{0}$. Fix a p-group $S$ such that $S_{0} \triangleleft S$ and $\operatorname{Aut}_{S}\left(S_{0}\right) \leq \operatorname{Aut}_{\text {fus }}\left(S_{0}, \mathcal{F}_{0}\right)$, and an action of $S$ on $\mathcal{L}_{0}$ which:

(a) extends the conjugation action of $S_{0}$ on $\mathcal{L}_{0}$;

(b) makes the canonical monomorphism $\delta_{S_{0}}: S_{0} \longrightarrow$ Aut $_{\mathcal{L}_{0}}\left(S_{0}\right) S$-equivariant;

(c) makes the projection $\pi: \mathcal{L}_{0} \longrightarrow \mathcal{F}_{0} S$-equivariant with respect to the $\operatorname{Aut}_{S}\left(S_{0}\right)$-action on $\mathcal{F}_{0}$; and

(d) sends inclusion morphisms in $\mathcal{L}_{0}$ to inclusion morphisms.

Then there is a p-local finite group $(S, \mathcal{F}, \mathcal{L})$ such that $\mathcal{F} \supseteq \mathcal{F}_{0}, \mathcal{L}^{q} \supseteq \mathcal{L}_{0}$, the conjugation action of $S$ on $\mathcal{L}^{q}$ restricts to the given $S$-action on $\mathcal{L}_{0}$, and $\left(S_{0}, \mathcal{F}_{0}, \mathcal{L}_{0}\right)$ is a subgroup of p-power index in $(S, \mathcal{F}, \mathcal{L})$.

Proof. Set

$\mathcal{H}_{0}=\operatorname{Ob}\left(\mathcal{L}_{0}\right)=\left\{P \leq S_{0} \mid P\right.$ is $\mathcal{F}_{0}$-centric $\} \quad$ and $\quad \mathcal{H}=\left\{P \leq S \mid P \cap S_{0} \in \mathcal{H}_{0}\right\}$.

To simplify notation, for any $P \leq S$, we write $P_{0}=P \cap S_{0}$. For $g \in S$ and $\varphi \in \operatorname{Mor}_{\mathcal{L}_{0}}(P, Q)$, we write $g \varphi g^{-1} \in \operatorname{Mor}_{\mathcal{L}_{0}}\left(g P g^{-1}, g Q g^{-1}\right)$ for the given action of $g$ on $\varphi$. By (a), when $g \in S_{0}$, this agrees with the morphism $g \varphi g^{-1}$ already defined.

Step 1: We first define categories $\mathcal{L}_{1} \supseteq \mathcal{L}_{0}$ and $\mathcal{F}_{1} \supseteq \mathcal{F}_{0}$, where $\operatorname{Ob}\left(\mathcal{F}_{1}\right)=$ $\operatorname{Ob}\left(\mathcal{F}_{0}\right)$ and $\operatorname{Ob}\left(\mathcal{L}_{1}\right)=\mathcal{H}_{0}$. Set

$$
\operatorname{Mor}\left(\mathcal{L}_{1}\right)=S \times_{S_{0}} \operatorname{Mor}\left(\mathcal{L}_{0}\right)=\left(S \times \operatorname{Mor}\left(\mathcal{L}_{0}\right)\right) / \sim,
$$

where $\left(g g_{0}, \varphi\right) \sim\left(g, g_{0} \varphi\right)$ for $g \in S, g_{0} \in S_{0}$, and $\varphi \in \operatorname{Mor}\left(\mathcal{L}_{0}\right)$. If $\varphi \in \operatorname{Mor}_{\mathcal{L}_{0}}(P, Q)$, then $\llbracket g, \varphi \rrbracket \in \operatorname{Mor}_{\mathcal{L}_{1}}\left(P, g Q g^{-1}\right)$ denotes the equivalence class of the pair $(g, \varphi)$. Composition is defined by

$$
\llbracket g, \varphi \rrbracket \circ \llbracket h, \psi \rrbracket=\llbracket g h, h^{-1} \varphi h \circ \psi \rrbracket .
$$

Note that if $\varphi \in \operatorname{Mor}_{\mathcal{L}_{0}}(P, Q)$, then $h^{-1} \varphi h \in \operatorname{Mor}_{\mathcal{L}_{0}}\left(h^{-1} P h, h^{-1} Q h\right)$. To show that this is well defined, we note that for all $g, h \in S, g_{0}, h_{0} \in S_{0}$, and $\varphi, \psi \in \operatorname{Mor}\left(\mathcal{L}_{0}\right)$ with appropriate domain and range,

$$
\begin{aligned}
& \llbracket g g_{0}, \varphi \rrbracket \circ \llbracket h h_{0}, \psi \rrbracket=\llbracket g g_{0} h h_{0}, h_{0}^{-1}\left(h^{-1} \varphi h\right) h_{0} \circ \psi \rrbracket=\llbracket g h \cdot\left(h^{-1} g_{0} h\right),\left(h^{-1} \varphi h\right) \circ h_{0} \psi \rrbracket \\
& =\llbracket g h,\left(h^{-1} g_{0} h\right) \cdot\left(h^{-1} \varphi h\right) \circ h_{0} \psi \rrbracket=\llbracket g h, h^{-1}\left(g_{0} \varphi\right) h \circ h_{0} \psi \rrbracket=\llbracket g, g_{0} \varphi \rrbracket \circ \llbracket h, h_{0} \psi \rrbracket .
\end{aligned}
$$

Here, the second to last equality follows from assumptions (b) and (d).

Let $\mathcal{F}_{1}$ be the smallest fusion system over $S_{0}$ which contains $\mathcal{F}_{0}$ and $\operatorname{Aut}_{S}\left(S_{0}\right)$. By assumption, $\operatorname{Aut}_{S}\left(S_{0}\right) \leq \operatorname{Aut}_{\text {fus }}\left(S_{0}, \mathcal{F}_{0}\right)$. Thus for each $g \in S, c_{g}$ normalizes the fusion system $\mathcal{F}_{0}$ : for each $\varphi \in \operatorname{Mor}\left(\mathcal{F}_{0}\right)$ there is $\varphi^{\prime} \in \operatorname{Mor}\left(\mathcal{F}_{0}\right)$ such that $\varphi \circ c_{g}=c_{g} \circ \varphi^{\prime}$. Hence each morphism in $\mathcal{F}_{1}$ has the form $c_{g} \circ \varphi$ for some $g \in S$ and $\varphi \in \operatorname{Aut}\left(\mathcal{F}_{0}\right)$. Define

$$
\pi_{\mathcal{L}_{1}}: \mathcal{L}_{1} \longrightarrow \mathcal{F}_{1}
$$

by sending $\pi_{\mathcal{L}_{1}}(\llbracket g, \varphi \rrbracket)=c_{g} \circ \pi_{0}(\varphi)$, where $\pi_{0}$ denotes the natural projection from $\mathcal{L}_{0}$ to $\mathcal{F}_{0}$. This is a functor by (c).

For all $P, Q \in \mathcal{H}_{0}$, define

$$
\widehat{\delta}_{P, Q}: N_{S}(P, Q) \longrightarrow \operatorname{Mor}_{\mathcal{L}_{1}}(P, Q)
$$

by setting $\widehat{\delta}_{P, Q}(g)=\llbracket g, \iota_{P}^{g^{-1} Q g} \rrbracket$. This extends the canonical monomorphism $\delta_{P, Q}$ defined from $N_{S_{0}}(P, Q)$ to $\operatorname{Mor}_{\mathcal{L}_{0}}(P, Q)$. To simplify the notation below, we sometimes write $\widehat{x}=\widehat{\delta}_{P, Q}(x)$ for $x \in N_{S}(P, Q)$. 
Step 2: We next construct categories $\mathcal{L}_{2}$ and $\mathcal{F}_{2}$, both of which have object sets $\mathcal{H}$, and which contain $\mathcal{L}_{1}$ and the restriction of $\mathcal{F}_{1}$ to $\mathcal{H}_{0}$, respectively. Afterwards, we let $\mathcal{F}$ be the fusion system over $S$ generated by $\mathcal{F}_{2}$ and restrictions of morphisms.

Before doing this, we need to know that the following holds for each $P, Q \in \mathcal{H}_{0}$ and each $\psi \in \operatorname{Mor}_{\mathcal{L}_{1}}(P, Q)$ :

(1) $\forall x \in N_{S}(P)$ there is at most one $y \in N_{S}(Q)$ such that $\widehat{y} \circ \psi=\psi \circ \widehat{x}$.

Since $\psi$ is the composite of an isomorphism and an inclusion (and the claim clearly holds if $\psi$ is an isomorphism), it suffices to prove this when $P \leq Q$ and $\psi$ is the inclusion. We will show that we must have $y=x$ in that case. By definition,

$$
\iota_{P}^{Q} \circ \widehat{x}=\llbracket 1, \iota_{P}^{Q} \rrbracket \circ \llbracket x, \operatorname{Id}_{P} \rrbracket=\llbracket x, \iota_{P}^{x^{-1} Q x} \rrbracket \quad \text { and } \quad \widehat{y} \circ \iota_{P}^{Q}=\llbracket y, \iota_{P}^{Q} \rrbracket
$$

(since conjugation sends inclusions to inclusions). So if $\widehat{y} \circ \iota_{P}^{Q}=\iota_{P}^{Q} \circ \widehat{x}$, then there exists $g_{0} \in S_{0}$ such that $y=x g_{0}$ and $\iota_{P}^{Q}=g_{0}^{-1} \iota_{P}^{x^{-1} Q x}$, and thus

$$
\delta_{P, Q}(1)=\iota_{P}^{Q}=g_{0}^{-1} \iota_{P}^{x^{-1} Q x}=\delta_{x^{-1} Q x, Q}\left(g_{0}^{-1}\right) \circ \delta_{P, x^{-1} Q x}(1)=\delta_{P, Q}\left(g_{0}^{-1}\right) .
$$

Hence $g_{0}=1$ by the injectivity of $\delta_{P, Q}$ in Proposition 1.13, and so $\widehat{y} \circ \iota_{P}^{Q}=\iota_{P}^{Q} \circ \widehat{x}$ only if $x=y \in N_{S}(Q)$.

Now let $\mathcal{L}_{2}$ be the category with $\operatorname{Ob}\left(\mathcal{L}_{2}\right)=\mathcal{H}$, and where for all $P, Q \in \mathcal{H}$,

$$
\operatorname{Mor}_{\mathcal{L}_{2}}(P, Q)=\left\{\psi \in \operatorname{Mor}_{\mathcal{L}_{1}}\left(P_{0}, Q_{0}\right) \mid \forall x \in P \exists y \in Q \text { such that } \psi \circ \widehat{x}=\widehat{y} \circ \psi\right\} .
$$

Let

$$
\widehat{\delta}_{P, Q}: \underset{\subseteq N_{S}(P, Q)}{N_{S}\left(P_{0}\right)} \longrightarrow \longrightarrow \operatorname{Mor}_{\mathcal{L}_{2}}(P, Q)
$$

be the restriction of $\widehat{\delta}_{P_{0}, Q_{0}}$. Let $\mathcal{F}_{2}$ be the category with $\operatorname{Ob}\left(\mathcal{F}_{2}\right)=\mathcal{H}$, and where $\operatorname{Mor}_{\mathcal{F}_{2}}(P, Q)=\left\{\varphi \in \operatorname{Hom}(P, Q) \mid \exists \psi \in \operatorname{Mor}_{\mathcal{L}_{2}}(P, Q)\right.$ such that $\left.\psi \circ \widehat{x}=\widehat{\varphi(x)} \circ \psi \forall x \in P\right\}$. Let $\pi: \mathcal{L}_{2} \rightarrow \mathcal{F}_{2}$ be the functor which sends $\psi \in \operatorname{Mor}_{\mathcal{L}_{2}}(P, Q)$ to the homomorphism $\pi(\psi)(x)=y$ if $\psi \circ \widehat{x}=\widehat{y} \circ \psi$ (uniquely defined by $(1)$ ). Let $\mathcal{F}$ be the fusion system over $S$ generated by $\mathcal{F}_{2}$ and restriction of homomorphisms.

Set $\Gamma=S / S_{0}$. Let

$$
\widehat{\theta}: \mathcal{L}_{2} \longrightarrow \mathcal{B}(\Gamma)
$$

be the functor defined by setting $\widehat{\theta}(\llbracket g, \varphi \rrbracket)=g S_{0}$. In particular, $\left.\left(\theta^{-1}(1)\right)\right|_{\mathcal{H}_{0}}=\mathcal{L}_{0}$.

Step 3: We next show that each $P \in \mathcal{H}$ is $\mathcal{F}$-conjugate to a subgroup $P^{\prime}$ such that $P_{0}^{\prime}$ is fully normalized in $\mathcal{F}_{0}$. Moreover, we show that $P^{\prime}$ can be chosen so that the following holds:

$$
\forall g \in S \text { such that } g P_{0} g^{-1} \text { is } \mathcal{F}_{0} \text {-conjugate to } P_{0}, g \cdot S_{0} \cap N_{S}\left(P_{0}^{\prime}\right) \neq \emptyset .
$$

To see this, let $\mathcal{P}_{\text {fn }}$ be the set of all $S_{0}$-conjugacy classes $\left[P_{0}^{\prime}\right]$ of subgroups $P_{0}^{\prime} \leq S_{0}$ which are $\mathcal{F}_{0}$-conjugate to $P_{0}$ and fully normalized in $\mathcal{F}_{0}$. (If $P_{0}^{\prime}$ is fully normalized in $\mathcal{F}_{0}$, then so is every subgroup in $\left[P_{0}^{\prime}\right]$.) Let $S^{\prime} \subseteq S$ be the subset of elements $g \in S$ such that $g P_{0} g^{-1}$ is $\mathcal{F}_{0}$-conjugate to $P_{0}$. In particular, $S^{\prime} \geq N_{S}\left(P_{0}\right) \geq P$. Since each $g \in S$ acts on $\mathcal{F}_{0}$ - two subgroups $Q, Q^{\prime} \leq S_{0}$ are $\mathcal{F}_{0}$-conjugate if and only if $g Q g^{-1}$ and $g Q^{\prime} g^{-1}$ are $\mathcal{F}_{0}$-conjugate $-S^{\prime}$ is a subgroup of $S$.

For all $g \in S^{\prime}$ and $\left[P_{0}^{\prime}\right] \in \mathcal{P}_{\mathrm{fn}}, g P_{0}^{\prime} g^{-1}$ is $\mathcal{F}_{0}$-conjugate to $g P_{0} g^{-1}$ and hence to $P_{0}$, and is fully normalized since $g$ normalizes $S_{0}$. Thus $S^{\prime} / S_{0}$ acts on $\mathcal{P}_{\text {fn }}$, and this set has order prime to $p$ by Proposition 1.16. So we can thus choose a subgroup $P_{0}^{\prime} \leq S_{0}$ such that $\left[P_{0}^{\prime}\right] \in \mathcal{P}_{\text {fn }}$ and is fixed by $S^{\prime}$. In particular, $P_{0}^{\prime}$ is fully normalized in $\mathcal{F}_{0}$. 
Also, for each $g \in S^{\prime}$, some element of $g \cdot S_{0}$ normalizes $P_{0}^{\prime}$ (since $\left[P_{0}^{\prime}\right]=\left[g P_{0}^{\prime} g^{-1}\right]$ ) - and this proves (2).

Now consider the set $\operatorname{Rep}_{\mathcal{F}_{0}}\left(P_{0}, S_{0}\right)=\operatorname{Hom}_{\mathcal{F}_{0}}\left(P_{0}, S_{0}\right) / \operatorname{Inn}\left(S_{0}\right)$. Since $P \leq S^{\prime}$, the group $P / P_{0}$ acts on this set by conjugation (i.e., $g P_{0} \in P / P_{0}$ acts on $[\varphi]$, for $\varphi \in \operatorname{Hom}_{\mathcal{F}_{0}}\left(P_{0}, S_{0}\right)$, by sending it to $\left.\left[c_{g} \varphi c_{g}^{-1}\right]\right)$. In particular, since the $S_{0^{-}}$ conjugacy class $\left[P_{0}^{\prime}\right]$ is invariant under conjugation by $P / P_{0}$, this group leaves invariant the subset $X \subseteq \operatorname{Rep}_{\mathcal{F}_{0}}\left(P_{0}, S_{0}\right)$ of all conjugacy classes $[\varphi]$ of homomorphisms such that $[\operatorname{Im}(\varphi)]=\left[P_{0}^{\prime}\right]$. Fix any $\varphi \in \operatorname{Iso}_{\mathcal{F}_{0}}\left(P_{0}, P_{0}^{\prime}\right)$ (recall that subgroups in $\mathcal{P}_{\text {fn }}$ are $\mathcal{F}_{0}$-conjugate to $P_{0}$ ). Every element of $X$ has the form $[\alpha \varphi]$ for some $\alpha \in \operatorname{Aut}_{\mathcal{F}_{0}}\left(P_{0}^{\prime}\right)$, and $[\alpha \varphi]=[\beta \varphi]$ if and only if $\alpha \beta^{-1} \in \operatorname{Aut}_{S_{0}}\left(P_{0}^{\prime}\right)$. Thus $|X|=\left|\operatorname{Aut}_{\mathcal{F}_{0}}\left(P_{0}^{\prime}\right)\right| /\left|\operatorname{Aut}_{S_{0}}\left(P_{0}^{\prime}\right)\right|$, and is prime to $p$ since $P_{0}^{\prime}$ is fully normalized in $\mathcal{F}_{0}$. We can thus choose $\varphi_{0} \in \operatorname{Hom}_{\mathcal{F}_{0}}\left(P_{0}, S_{0}\right)$ such that $\varphi_{0}\left(P_{0}\right)=P_{0}^{\prime}$ and $\left[\varphi_{0}\right]$ is invariant under the $P / P_{0}$-action.

Fix $\psi \in \operatorname{Iso}_{\mathcal{L}_{0}}\left(P_{0}, P_{0}^{\prime}\right)$ such that $\pi(\psi)=\varphi_{0}$. Since $\left[\varphi_{0}\right]$ is $P / P_{0}$-invariant, for each $x \in P$, there is some $y \in x \cdot S_{0}$ such that $c_{y} \circ \varphi_{0}=\varphi_{0} \circ c_{x}$. By $(\mathrm{A})_{q}$ (and since $\psi$ is an isomorphism), there is $y^{\prime} \in y \cdot C_{S_{0}}\left(P_{0}^{\prime}\right)$ such that $\widehat{y^{\prime}} \circ \psi=\psi \circ \widehat{x}$ in $\mathcal{L}_{1}$. This element $y^{\prime}$ is unique by (1); and upon setting $\varphi(x)=y^{\prime}$ we get a homomorphism $\varphi \in \operatorname{Hom}_{\mathcal{F}}(P, S)$ which extends $\varphi_{0}$. Set $P^{\prime}=\varphi(P)$; then $P^{\prime}$ is $\mathcal{F}$-conjugate to $P$ and $P_{0}^{\prime}=P^{\prime} \cap S_{0}$.

Step 4: In Step 5, we will prove that $\mathcal{F}$ is saturated, using [5A1, Theorem 2.2]. Before that theorem can be applied, a certain technical condition must be checked.

Assume that $P$ is $\mathcal{F}$-centric, but not in $\mathcal{H}$. By Step $3, P$ is $\mathcal{F}$-conjugate to some $P^{\prime}$ such that $P_{0}^{\prime}$ is fully normalized in $\mathcal{F}_{0}$. Thus $P_{0}^{\prime}$ is fully centralized in $\mathcal{F}_{0}$ and not $\mathcal{F}_{0}$-centric, which implies that $C_{S_{0}}\left(P_{0}^{\prime}\right) \not \leq P_{0}^{\prime}$. Then $P^{\prime}$ acts on $C_{S_{0}}\left(P_{0}^{\prime}\right) \cdot P_{0}^{\prime} / P_{0}^{\prime}$ with fixed subgroup $Q P_{0}^{\prime} / P_{0}^{\prime} \neq 1$ for some $Q \leq C_{S_{0}}\left(P_{0}^{\prime}\right)$, and $\left[Q, P^{\prime}\right] \leq P_{0}^{\prime}$ since $P^{\prime} / P_{0}^{\prime}$ centralizes $Q P_{0}^{\prime} / P_{0}^{\prime}$. Hence $Q \not \leq P_{0}^{\prime}$, and $Q \leq N_{S}\left(P^{\prime}\right)$ since $\left[Q, P^{\prime}\right] \leq P^{\prime}$. For any $x \in Q \backslash P_{0}^{\prime},\left[c_{x}\right] \neq 1 \in \operatorname{Out}\left(P^{\prime}\right)\left(C_{S}\left(P^{\prime}\right) \leq P^{\prime}\right.$ since $P$ is $\mathcal{F}$-centric), but $c_{x}$ induces the identity on $P_{0}^{\prime}$ (since $\left.Q \leq C_{S_{0}}\left(P_{0}^{\prime}\right)\right)$ and on $P^{\prime} / P_{0}^{\prime}$ (since $x \in S_{0}$ ). Hence $\left[c_{x}\right] \in O_{p}\left(\operatorname{Out}_{\mathcal{F}}\left(P^{\prime}\right)\right)$ by Lemma 1.15. This shows that

(3)

$P \mathcal{F}$-centric, $P \notin \mathcal{H} \Longrightarrow \exists P^{\prime} \mathcal{F}$-conjugate to $P$, $\operatorname{Out}_{S}\left(P^{\prime}\right) \cap O_{p}\left(\operatorname{Out}_{\mathcal{F}}\left(P^{\prime}\right)\right) \neq 1$.

Step 5: We next show that $\mathcal{F}$ is saturated, and also (since it will be needed in the proof of $(\mathrm{II})$ ) that axiom $(\mathrm{A})_{q}$ holds for $\mathcal{L}_{2}$. By [5A1, Theorem 2.2] (the stronger form of Theorem $1.5(\mathrm{~b})$ ), it suffices to prove that the subgroups in $\mathcal{H}$ satisfy the axioms for saturation. Note in particular that condition $(*)$ in [5A1, Theorem 2.2] is precisely what is shown in (3).

Proof of (I). Fix a subgroup $P \in \mathcal{H}$ which is fully normalized in $\mathcal{F}$. Let $S^{\prime} / S_{0} \leq$ $S / S_{0}$ be the stabilizer of the $\mathcal{F}_{0}$-conjugacy class of $P_{0}$. By Step $3, P$ is $\mathcal{F}$-conjugate to a subgroup $P^{\prime}$ such that $P_{0}^{\prime}$ is fully normalized in $\mathcal{F}_{0}$; and such that for each $g \in S^{\prime}$, some element of $g \cdot S_{0}$ normalizes $P_{0}^{\prime}$ (see (2)). Hence there are short exact sequences

$$
\begin{gathered}
1 \longrightarrow \operatorname{Aut}_{\mathcal{L}_{0}}\left(P_{0}^{\prime}\right) \longrightarrow \operatorname{Aut}_{\mathcal{L}_{1}}\left(P_{0}^{\prime}\right) \longrightarrow S^{\prime} / S_{0} \longrightarrow 1, \\
\left.1 \longrightarrow N_{S_{0}}\left(P_{0}^{\prime}\right) \longrightarrow P_{0}^{\prime}\right) \longrightarrow S_{0} \longrightarrow 1
\end{gathered}
$$

We consider $P^{\prime} \leq N_{S}\left(P_{0}^{\prime}\right)$ as subgroups of $\operatorname{Aut}_{\mathcal{L}_{1}}\left(P_{0}^{\prime}\right)$ via $\widehat{\delta}_{P_{0}^{\prime}, P_{0}^{\prime}}$. Then

$$
\left[\operatorname{Aut}_{\mathcal{L}_{1}}\left(P_{0}^{\prime}\right): N_{S}\left(P_{0}^{\prime}\right)\right]=\left[\operatorname{Aut}_{\mathcal{L}_{0}}\left(P_{0}^{\prime}\right): N_{S_{0}}\left(P_{0}^{\prime}\right)\right]
$$


is prime to $p$ since $P_{0}^{\prime}$ is fully normalized in $\mathcal{F}_{0}$, and so $N_{S}\left(P_{0}^{\prime}\right) \in \operatorname{Syl}_{p}\left(\operatorname{Aut}_{\mathcal{L}_{1}}\left(P_{0}^{\prime}\right)\right.$ ). Fix $\psi \in \operatorname{Aut}_{\mathcal{L}_{1}}\left(P_{0}^{\prime}\right)$ such that $\psi^{-1} N_{S}\left(P_{0}^{\prime}\right) \psi$ contains a Sylow $p$-subgroup of the

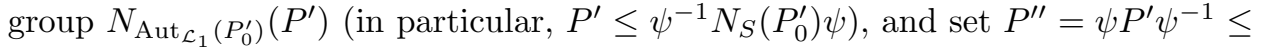
$N_{S}\left(P_{0}^{\prime}\right)$. Then $\psi \in \operatorname{Iso}_{\mathcal{L}_{2}}\left(P^{\prime}, P^{\prime \prime}\right)$ by definition of $\mathcal{L}_{2}$. In particular, $P^{\prime \prime}$ is $\mathcal{F}$ conjugate to $P$, and $P_{0}^{\prime \prime}=P_{0}^{\prime}$. Also, $N_{S}\left(P_{0}^{\prime}\right)$ contains a Sylow $p$-subgroup of $N_{\text {Aut }_{\mathcal{L}_{1}}\left(P_{0}^{\prime}\right)}\left(P^{\prime \prime}\right)$,

$$
\operatorname{Aut}_{\mathcal{L}_{2}}\left(P^{\prime \prime}\right)=N_{\operatorname{Aut}_{\mathcal{L}_{1}}\left(P_{0}^{\prime}\right)}\left(P^{\prime \prime}\right) \quad \text { and } \quad N_{S}\left(P^{\prime \prime}\right)=N_{N_{S}\left(P_{0}^{\prime}\right)}\left(P^{\prime \prime}\right),
$$

and it follows that $N_{S}\left(P^{\prime \prime}\right) \in \operatorname{Syl}_{p}\left(\operatorname{Aut}_{\mathcal{L}_{2}}\left(P^{\prime \prime}\right)\right)$.

Now, $\operatorname{Aut}_{\mathcal{L}_{2}}(P) \cong \operatorname{Aut}_{\mathcal{L}_{2}}\left(P^{\prime \prime}\right)$ since $P$ and $P^{\prime \prime}$ are $\mathcal{F}$-conjugate, and $\left|N_{S}(P)\right| \geq$ $\left|N_{S}\left(P^{\prime \prime}\right)\right|$ since $P$ is fully normalized. Thus $N_{S}(P) \in \operatorname{Syl}_{p}\left(\operatorname{Aut}_{\mathcal{L}_{2}}(P)\right)$, and hence $\operatorname{Aut}_{S}(P) \in \operatorname{Syl}_{p}\left(\operatorname{Aut}_{\mathcal{F}}(P)\right)$. Also, $N_{S}(P)$ contains the kernel of the projection from $\operatorname{Aut}_{\mathcal{L}_{2}}(P)$ to $\operatorname{Aut}_{\mathcal{F}}(P)$; i.e., $C_{S}(P)$ is isomorphic to this kernel. For all $Q$ which is $\mathcal{F}$-conjugate to $P, C_{S}(Q)$ is isomorphic to a subgroup of the same kernel, so $\left|C_{S}(Q)\right| \leq\left|C_{S}(P)\right|$, and thus $P$ is fully centralized in $\mathcal{F}$.

Proof of $(A)_{q}$ for $\mathcal{L}_{2}$. It is clear from the construction that for any $P, Q \in \mathcal{H}$, $C_{S}(P)$ acts freely on $\operatorname{Mor}_{\mathcal{L}_{2}}(P, Q)$ via $\widehat{\delta}_{P, P}$. So it remains to show that when $P$ is fully centralized in $\mathcal{F}$, then for all $\psi, \psi^{\prime} \in \operatorname{Mor}_{\mathcal{L}_{2}}(P, Q)$ such that $\pi(\psi)=\pi\left(\psi^{\prime}\right)$, there is some $x \in C_{S}(P)$ such that $\psi^{\prime}=\psi \circ \widehat{x}$. Since every morphism in $\mathcal{L}_{2}$ is the composite of an isomorphism followed by an inclusion, it suffices to show this when $\psi$ and $\psi^{\prime}$ are isomorphisms. But in this case, $\psi^{-1} \psi^{\prime} \in \operatorname{Aut}_{\mathcal{L}_{2}}(P)$ lies in the kernel of the map to $\operatorname{Aut}_{\mathcal{F}}(P)$. We have just seen, in the proof of $(\mathrm{I})$, that this implies there is some $x \in C_{S}(P)$ such that $\widehat{x}=\psi^{-1} \psi^{\prime}$, so $\psi^{\prime}=\psi \circ \widehat{x}$, and this is what we wanted to prove.

Proof of (II). Fix $\varphi \in \operatorname{Hom}_{\mathcal{F}}(P, S)$, where $\varphi(P)$ is fully centralized in $\mathcal{F}$. Set $P^{\prime}=\varphi(P)$. By definition of $\mathcal{F}_{2} \subseteq \mathcal{F}$ and of $\mathcal{L}_{2}$, there is some $\psi \in \operatorname{Iso}_{\mathcal{L}_{2}}\left(P, P^{\prime}\right) \subseteq$ Iso $_{\mathcal{L}_{1}}\left(P_{0}, P_{0}^{\prime}\right)$ such that $\psi \circ \widehat{g}=\widehat{\varphi(g)} \circ \psi$ for all $g \in P$. Upon replacing $\psi$ by $\widehat{x} \circ \psi$ for some appropriate $x \in S$ (and replacing $\varphi$ by $c_{x} \circ \varphi$ and $P^{\prime}$ by $x P^{\prime} x^{-1}$ ), we can assume that $\psi \in \operatorname{Iso}_{\mathcal{L}_{0}}\left(P_{0}, P_{0}^{\prime}\right)$ and $\left.\varphi\right|_{P_{0}} \in \operatorname{Hom}_{\mathcal{F}_{0}}\left(P_{0}, S_{0}\right)$.

Consider the subgroups

$$
\begin{aligned}
N_{\varphi} & =\left\{x \in N_{S}(P) \mid \varphi c_{x} \varphi^{-1} \in \operatorname{Aut}_{S}\left(P^{\prime}\right)\right\}, \\
N^{\prime}=N_{\varphi \mid P_{0}} \cap N_{\varphi} & =\left\{x \in N_{\varphi} \cap S_{0}\left|\left(\varphi c_{x} \varphi^{-1}\right)\right|_{P_{0}} \in \operatorname{Aut}_{S_{0}}\left(P_{0}^{\prime}\right)\right\} .
\end{aligned}
$$

We will see shortly that $N^{\prime}=N_{\varphi} \cap S_{0}$. By (II) applied to the saturated fusion system $\mathcal{F}_{0}$, there is $\bar{\varphi}_{0} \in \operatorname{Hom}_{\mathcal{F}_{0}}\left(N^{\prime}, S\right)$ which extends $\left.\varphi\right|_{P_{0}}$, and it lifts to $\bar{\psi} \in$ $\operatorname{Hom}_{\mathcal{L}_{0}}\left(N^{\prime}, S\right)$. By (A) (applied to $\left.\mathcal{L}_{0}\right)$, there is $z \in Z\left(P_{0}\right)$ such that $\psi=\left(\left.\bar{\psi}\right|_{P_{0}}\right) \circ \widehat{z}$. Upon replacing $\bar{\psi}$ by $\bar{\psi} \circ \widehat{z}$ (and $\bar{\varphi}_{0}$ by $\bar{\varphi}_{0} \circ c_{z}$ ), we can assume that $\psi=\left.\bar{\psi}\right|_{P_{0}}$.

For any $x \in N_{\varphi} \cap S_{0}, \varphi c_{x} \varphi^{-1}=c_{y} \in \operatorname{Aut}_{\mathcal{F}}\left(P^{\prime}\right)$ for some $y \in N_{S}\left(P^{\prime}\right)$. Hence by $(\mathrm{A})_{q}\left(\right.$ for $\left.\mathcal{L}_{2}\right), \psi \widehat{x} \psi^{-1}=\widehat{y z}$ for some unique $z \in C_{S}\left(P^{\prime}\right)$. Thus $\widehat{y z} \in \operatorname{Aut}_{\mathcal{L}_{0}}\left(P_{0}^{\prime}\right)$, and by definition of the distinguished monomorphisms for $\mathcal{L}_{1}$, this is possible only if $y z \in S_{0}$. Thus $\varphi c_{x} \varphi^{-1}=c_{y z}$ where $y z \in N_{S_{0}}\left(P^{\prime}\right)$, and so $x \in N^{\prime}$. This shows that $N^{\prime}=N_{\varphi} \cap S_{0}$.

Define $\bar{\varphi} \in \operatorname{Hom}\left(N_{\varphi}, S\right)$ by the relation $\varphi(x)=y$ if $\psi \widehat{x} \psi^{-1}=\widehat{y}$. In particular, this implies that $y x^{-1}=\psi \circ\left(x \psi x^{-1}\right)^{-1}$ is a morphism in $\mathcal{L}_{0}$, and hence that $y x^{-1} \in S_{0}$. Also, $\left.\bar{\varphi}\right|_{P}=\varphi$ by the original assumption on $\psi$. It remains to show 
that $\bar{\varphi} \in \operatorname{Hom}_{\mathcal{F}}\left(N_{\varphi}, S\right)$. To do this, it suffices to show that $\bar{\psi} \circ \widehat{x}=\widehat{y} \circ \bar{\psi}$ in $\operatorname{Mor}_{\mathcal{L}_{2}}\left(N^{\prime}, S\right)$ for all $x$, where $y=\bar{\varphi}(x)$. Equivalently, we must show that

$$
\bar{\psi}=\widehat{y x^{-1}} \circ\left(x \bar{\psi} x^{-1}\right) .
$$

Since $y x^{-1} \in S_{0}$, both sides in (4) are in $\mathcal{L}_{0}$, and they are equal after restriction to $P_{0}$. Hence they are equal as morphisms defined on $N_{\varphi} \cap S_{0}$ by [5A1, Lemma 3.9], and this finishes the proof.

Step 6: We next check that $\mathcal{F}_{0}$ has $p$-power index in $\mathcal{F}$. For any $P \leq S$ and any $\alpha \in \operatorname{Aut}_{\mathcal{F}}(P)$ of order prime to $P, \alpha$ induces the identity on $P / P_{0}$ by construction, and hence $x^{-1} \alpha(x) \in S_{0}$ for all $x \in P$. This shows that $S_{0} \geq O_{\mathcal{F}}^{p}(S)$ (see Definition 2.1). Also, by construction, for all $P \leq S_{0}, \operatorname{Aut}_{\mathcal{F}_{0}}(P)$ is normal of $p$-power index in $\operatorname{Aut}_{\mathcal{F}}(P)$, and thus contains $O^{p}\left(\operatorname{Aut}_{\mathcal{F}}(P)\right)$. This proves that the fusion subsystem $\mathcal{F}_{0}$ has $p$-power index in $\mathcal{F}$ in the sense of Definition 3.1 .

Step 7: It remains to construct a quasicentric linking system $\mathcal{L}^{q}$ which contains $\mathcal{L}_{2}$ as a full subcategory, and which is associated to $\mathcal{F}$. Note first that the axioms of Definition 1.9 are all satisfied by $\mathcal{L}_{2}$ : axiom $(\mathrm{A})_{q}$ holds by Step 5 , while axioms $(\mathrm{B})_{q},(\mathrm{C})_{q}$, and $(\mathrm{D})_{q}$ follow directly from the construction in Steps 1 and 2.

Let $\mathcal{L}_{2}^{c} \subseteq \mathcal{L}_{2}$ be the full subcategory whose objects are the set $\mathcal{H}^{c} \subseteq \mathcal{H}$ of subgroups in $\mathcal{H}$ which are $\mathcal{F}$-centric. We first construct a centric linking system $\mathcal{L} \supseteq \mathcal{L}_{2}^{c}$ associated to $\mathcal{F}$. For any set $\mathcal{K}$ of $\mathcal{F}$-centric subgroups of $S$, let $\mathcal{O}^{c}(\mathcal{F})$ be the orbit category of $\mathcal{F}$, let $\mathcal{O}^{\mathcal{K}}(\mathcal{F}) \subseteq \mathcal{O}^{c}(\mathcal{F})$ be the full subcategory with object set $\mathcal{K}$, and let $\mathcal{Z}_{\mathcal{F}}^{\mathcal{K}}$ be the functor $P \mapsto Z(P)$ on $\mathcal{O}^{\mathcal{K}}(\mathcal{F})$ which sends (see Definition 1.7 for more detail). By [BLO2, Proposition 3.1], when $\mathcal{K}$ is closed under $\mathcal{F}$-conjugacy and overgroups, the obstruction to the existence of a linking system with object set $\mathcal{K}$ lies in $\lim ^{3}\left(\mathcal{Z}_{\mathcal{F}}^{\mathcal{K}}\right)$, and the obstruction to its uniqueness lies in $\lim ^{2}\left(\mathcal{Z}_{\mathcal{F}}^{\mathcal{K}}\right)$. Furthermore, by [BLO2, Proposition 3.2], if $\mathcal{P}$ is an $\mathcal{F}$-conjugacy class of $\mathcal{F}$-centric subgroups maximal among those not in $\mathcal{K}$, and $\mathcal{K}^{\prime}=\mathcal{K} \cup \mathcal{P}$, then the inclusion of functors induces an isomorphism between the higher limits of $\mathcal{Z}_{\mathcal{F}}^{\mathcal{K}}$ and $\mathcal{Z}_{\mathcal{F}}^{\mathcal{K}^{\prime}}$ if certain groups $\Lambda^{*}\left(\operatorname{Out}_{\mathcal{F}}(P) ; Z(P)\right)$ vanish for $P \in \mathcal{P}$. By $(3), O_{p}\left(\operatorname{Out}_{\mathcal{F}}(P)\right) \neq 1$ for any $\mathcal{F}_{0}$-centric subgroup $P \notin \mathcal{H}^{c}$, and hence $\Lambda^{*}\left(\operatorname{Out}_{\mathcal{F}}(P) ; Z(P)\right)=0$ for such $P$ by [JMO, Proposition 6.1(ii)]. So these obstructions all vanish, and there is a centric linking system $\mathcal{L} \supseteq \mathcal{L}_{2}^{c}$ associated to $\mathcal{F}$.

Now let $\mathcal{L}^{q}$ be the quasicentric linking system associated to $(S, \mathcal{F}, \mathcal{L})$. For each $P \in \mathcal{H}=\operatorname{Ob}\left(\mathcal{L}_{2}\right), P \cap S_{0}$ is $\mathcal{F}_{0}$-centric by definition, hence is $\mathcal{F}$-quasicentric by Theorem 4.3(a), and thus $P$ is also $\mathcal{F}$-quasicentric. Also, $\mathcal{H}$ contains all $\mathcal{F}$-centric $\mathcal{F}$-radical subgroups by $(3)$, and $\mathcal{H}$ is closed under $\mathcal{F}$-conjugacy and overgroups (by definition of $\mathcal{F}$ and $\mathcal{H}$ ). Hence by [5A1, Proposition 3.12], since $\mathcal{L}_{2}^{c}$ is a full subcategory of $\mathcal{L}$ by construction, $\mathcal{L}_{2}$ is isomorphic to a full subcategory of $\mathcal{L}^{q}$ in a way which preserves the projection functors and distinguished monomorphisms. So $\mathcal{L}_{0}$ can also be identified with a linking subsystem of $\mathcal{L}^{q}$, and this finishes the proof.

We now prove a topological version of Theorem 4.6, By Theorem 4.4, for any inclusion $\left(S_{0}, \mathcal{F}_{0}, \mathcal{L}_{0}\right) \subseteq(S, \mathcal{F}, \mathcal{L})$ of $p$-local finite groups of $p$-power index, where $S_{0} \triangleleft S$ and $\Gamma=S / S_{0}$, there is a fibration sequence $\left|\mathcal{L}_{0}\right|_{p}^{\wedge} \longrightarrow|\mathcal{L}|_{p}^{\wedge} \longrightarrow B \Gamma$. So it is natural to ask whether the opposite is true: given a fibration sequence whose base is the classifying space of a finite $p$-group, and whose fiber is the classifying 
space of a $p$-local finite group, is the total space also the classifying space of a $p$-local finite group? The next proposition shows that this is, in fact, the case.

Before stating the proposition, we first define some categories which will be needed in its proof. Fix a space $Y$, a $p$-group $S$, and a map $f: B S \longrightarrow Y$. For $P \leq S$, we regard $B P$ as a subspace of $B S$; all of these subspaces contain the basepoint $* \in B S$. We define three categories in this situation, $\mathcal{F}_{S, f}(Y), \mathcal{L}_{S, f}(Y)$, and $\mathcal{M}_{S, f}(Y)$, all of which have as objects the subgroups of $S$. Of these, the first two are discrete categories, while $\mathcal{M}_{S, f}(Y)$ has a topology on its morphism sets. Morphisms in $\mathcal{F}_{S, f}(Y)$ are defined by setting

$$
\operatorname{Mor}_{\mathcal{F}_{S, f}(Y)}(P, Q)=\left\{\left.\varphi \in \operatorname{Hom}(P, Q)|f|_{B P} \simeq f\right|_{B Q} \circ B \varphi\right\}
$$

we think of this as the fusion category of $Y$ (with respect to $S$ and $f$ ).

Next define

$$
\begin{aligned}
\operatorname{Mor}_{\mathcal{M}_{S, f}(Y)}(P, Q)=\{(\varphi, H) \mid \varphi \in \operatorname{Hom}(P, Q), H: B P \times[0, t] & \rightarrow Y, \\
t & \left.\geq 0,\left.H\right|_{B P \times 0}=\left.f\right|_{B P},\left.H\right|_{B P \times t}=\left.f\right|_{B Q} \circ B \varphi\right\} .
\end{aligned}
$$

Thus a morphism in $\mathcal{M}_{S, f}(Y)$ has the form $(\varphi, H)$ where $H$ is a Moore homotopy in $Y$. Composition is defined by

$$
(\psi, K) \circ(\varphi, H)=(\psi \varphi,(K \circ(B \varphi \times \mathrm{Id})) \cdot H),
$$

where if $H$ and $K$ are homotopies parameterized by $[0, t]$ and $[0, s]$, respectively, then $(K \circ(B \varphi \times \mathrm{Id})) \cdot H$ is the composite homotopy parameterized by $[0, t+s]$.

Let $\mathcal{P}(Y)$ be the category of Moore paths in $Y$, and let $\operatorname{Res}_{*}: \mathcal{M}_{S, f}(Y) \rightarrow \mathcal{P}(Y)$ be the functor which sends each object to $f(*)$, and sends a morphism $(\varphi, H)$ to the path obtained by restricting $H$ to the basepoint $* \in B S$. Define a map ev from $|\mathcal{P}(Y)|$ to $Y$ as follows. For any $n$-simplex $\Delta^{n}$ in $|\mathcal{P}(Y)|$, indexed by a composable sequence of paths $\phi_{1}, \ldots, \phi_{n}$ where $\phi_{i}$ is defined on the interval $\left[0, t_{i}\right]$, let ev $\left.\right|_{\Delta^{n}}$ be the composite

$$
\Delta^{n} \stackrel{\lambda\left(t_{1}, \ldots, t_{n}\right)}{\longrightarrow}\left[0, t_{1}+\ldots+t_{n}\right] \stackrel{\phi_{n} \cdots \phi_{1}}{\longrightarrow} Y
$$

where $\lambda\left(t_{0}, \ldots, t_{n}\right)$ is the affine map which sends the $i$-th vertex to $t_{1}+\ldots+t_{i}$. The category $\mathcal{M}_{S, f}(Y)$ thus comes equipped with an "evaluation function"

$$
\text { eval: }\left|\mathcal{M}_{S, f}(Y)\right| \stackrel{\left|\operatorname{Res}_{*}\right|}{\longrightarrow}|\mathcal{P}(Y)| \stackrel{\text { ev }}{\longrightarrow} Y \text {. }
$$

Now set

$$
\operatorname{Mor}_{\mathcal{L}_{S, f}(Y)}(P, Q)=\pi_{0}\left(\operatorname{Mor}_{\mathcal{M}_{S, f}(Y)}(P, Q)\right) .
$$

We think of $\mathcal{L}_{S, f}(Y)$ as the linking category of $Y$. Also, for any set $\mathcal{H}$ of subgroups of $S$, we let $\mathcal{L}_{S, f}^{\mathcal{H}}(Y)$ and $\mathcal{M}_{S, f}^{\mathcal{H}}(Y)$ denote the full subcategories of $\mathcal{L}_{S, f}(Y)$ and $\mathcal{M}_{S, f}(Y)$ with object set $\mathcal{H}$. For more about the fusion and linking categories of a space, see [BLO2, $\S 7]$.

Theorem 4.7. Fix a p-local finite group $\left(S_{0}, \mathcal{F}_{0}, \mathcal{L}_{0}\right)$, a p-group $\Gamma$, and a fibration $X \stackrel{v}{\longrightarrow} B \Gamma$ with fiber $X_{0} \simeq\left|\mathcal{L}_{0}\right|_{p}^{\wedge}$. Then there is a p-local finite group $(S, \mathcal{F}, \mathcal{L})$ such that $S_{0} \triangleleft S, \mathcal{F}_{0} \subseteq \mathcal{F}$ is a fusion subsystem of $p$-power index, $S / S_{0} \cong \Gamma$, and $X \simeq|\mathcal{L}|_{p}^{\wedge}$.

Proof. Let $*$ denote the base point of $B \Gamma$, and assume $X_{0}=v^{-1}(*)$. Fix a homotopy equivalence $f:\left|\mathcal{L}_{0}\right|_{p}^{\wedge} \longrightarrow X_{0}$, regard $B S_{0}$ as a subspace of $\left|\mathcal{L}_{0}\right|$, and set $f_{0}=\left.f\right|_{B S_{0}}: B S_{0} \longrightarrow X_{0}$, also regarded as a map to $X$. Let $\mathcal{H}_{0}$ be the set of $\mathcal{F}_{0}$-centric subgroups of $S_{0}$. 
Step 1: By [BLO2, Proposition 7.3],

$$
\mathcal{F}_{0} \cong \mathcal{F}_{S_{0}, f_{0}}\left(X_{0}\right) \quad \text { and } \quad \mathcal{L}_{0} \cong \mathcal{L}_{S_{0}, f_{0}}^{\mathcal{H}_{0}}\left(X_{0}\right)
$$

We choose the inclusions $\iota_{P}^{Q} \in \operatorname{Mor}_{\mathcal{L}_{0}}(P, Q)$ (for $P \leq Q$ in $\mathcal{H}_{0}$ ) to correspond to the morphisms $\left(\operatorname{incl}_{P}^{Q},[c]\right)$ in $\mathcal{L}_{S_{0}, f_{0}}\left(X_{0}\right)$, where $c$ is the constant homotopy $\left.f_{0}\right|_{B P}$. Set

$$
\mathcal{F}_{1}=\mathcal{F}_{S_{0}, f_{0}}(X) \quad \text { and } \quad \mathcal{L}_{1}=\mathcal{L}_{S_{0}, f_{0}}^{\mathcal{H}_{0}}(X)
$$

where $f_{0}$ is now being regarded as a map $B S_{0} \longrightarrow X$. The inclusion $X_{0} \subseteq X$ makes $\mathcal{F}_{0}$ into a subcategory of $\mathcal{F}_{1}$ and $\mathcal{L}_{0}$ into a subcategory of $\mathcal{L}_{1}$.

For all $P \leq S_{0}$ which is fully centralized in $\mathcal{F}_{0}, \operatorname{Map}\left(B P, X_{0}\right)_{f_{0} \mid B P} \simeq\left|C_{\mathcal{L}_{0}}(P)\right|_{p}^{\wedge}$ by [BLO2, Theorem 6.3], where $C_{\mathcal{L}_{0}}(P)$ is a linking system over the centralizer $C_{S}(P)$. Since $P \in \mathcal{H}_{0}$ (i.e., $P$ is $\mathcal{F}_{0}$-centric) if and only if it is fully centralized and $C_{S_{0}}(P)=Z(P)$, this shows that

$$
P \in \mathcal{H}_{0} \Longleftrightarrow \operatorname{Map}\left(B P, X_{0}\right)_{\left.f_{0}\right|_{B P}} \simeq B Z(P) \text {. }
$$

If $P$ and $P^{\prime}$ are $\mathcal{F}_{1}$-conjugate, and $\varphi \in \operatorname{Iso}_{\mathcal{F}_{1}}\left(P, P^{\prime}\right)$, then the homotopy between $\left.f_{0}\right|_{B P}$ and $\left.f_{0}\right|_{B P^{\prime}} \circ B \varphi$ as maps from $B P$ to $X$ induces, using the homotopy lifting property for the fibration $v$, a homotopy between $\left.w \circ f_{0}\right|_{B P}$ and $\left.f_{0}\right|_{B P^{\prime}} \circ B \varphi$ (as maps from $B P$ to $X_{0}$ ), where $w: X_{0} \rightarrow \simeq X_{0}$ is the homotopy equivalence induced by lifting some loop in $B \Gamma$. Since $w$ is a homotopy equivalence and $B \varphi$ is a homeomorphism, this shows that the mapping spaces $\operatorname{Map}\left(B P, X_{0}\right)_{\left.f_{0}\right|_{B P}}$ and $\operatorname{Map}\left(B P^{\prime}, X_{0}\right)_{\left.f_{0}\right|_{B P^{\prime}}}$ are homotopy equivalent, and hence (by (1)) that $P^{\prime} \in \mathcal{H}_{0}$ if $P^{\prime} \in \mathcal{H}_{0}$. Thus for all $P, P^{\prime} \leq S_{0}$,

$$
P \mathcal{F}_{1} \text {-conjugate to } P^{\prime} \text { and } P \in \mathcal{H}_{0} \Longrightarrow P^{\prime} \in \mathcal{H}_{0} \text {. }
$$

Fix $S \in \operatorname{Syl}_{p}\left(\operatorname{Aut}_{\mathcal{L}_{1}}\left(S_{0}\right)\right)$. We identify $S_{0}$ as a subgroup of $S$ via the distinguished monomorphism $\delta_{S_{0}}$ from $S_{0}$ to $\operatorname{Aut}_{\mathcal{L}_{0}}\left(S_{0}\right) \leq \operatorname{Aut}_{\mathcal{L}_{1}}\left(S_{0}\right)$.

Step 2: For all $P \leq S$, and for $Y=X_{0}$ or $X$, we define

$$
\operatorname{Map}(B P, Y)_{\Phi}=\left\{f: B P \rightarrow Y \mid f \simeq f_{0} \circ B \varphi, \quad \varphi \in \operatorname{Hom}(P, S), \quad \varphi(P) \in \mathcal{H}_{0}\right\} .
$$

Using (2), we see that the fibration sequence $X_{0} \longrightarrow X \longrightarrow B \Gamma$ induces a fibration sequence of mapping spaces

$$
\operatorname{Map}\left(B P, X_{0}\right)_{\Phi} \longrightarrow \operatorname{Map}(B P, X)_{\Phi} \longrightarrow \operatorname{Map}(B P, B \Gamma)_{c t} \simeq B \Gamma,
$$

where $\operatorname{Map}(B P, B \Gamma)_{c t}$ is the space of null homotopic maps, and the last equivalence is induced by evaluation at the basepoint. By (1), each connected component of $\operatorname{Map}\left(B P, X_{0}\right)_{\Phi}$ is homotopy equivalent to $B Z(P)$, and hence the connected components of $\operatorname{Map}(B P, X)_{\Phi}$ are also aspherical.

For any morphism $(\varphi,[H]) \in \operatorname{Mor}_{\mathcal{L}_{1}}(P, Q)$, where $\varphi \in \operatorname{Hom}_{\mathcal{F}_{1}}(P, Q)$ and $[H]$ is the homotopy class of the path $H$ in $\operatorname{Map}(B P, X)_{\Phi}$, restricting $v \circ H$ to the basepoint of $B P$ defines a loop in $B \Gamma$, and thus an element of $\Gamma$. This defines a map from $\operatorname{Mor}\left(\mathcal{L}_{1}\right)$ to $\Gamma$ which sends composites to products, and thus a functor

$$
\widehat{\theta}: \mathcal{L}_{1} \longrightarrow \mathcal{B}(\Gamma) .
$$

By construction (and the fibration sequence (3)), for any $\psi \in \operatorname{Mor}\left(\mathcal{L}_{1}\right)$, we have $\psi \in \operatorname{Mor}\left(\mathcal{L}_{0}\right)$ if and only if $\widehat{\theta}(\psi)=1$.

Using the homotopy lifting property in (3), we see that $\widehat{\theta}$ restricts to a surjection of $\operatorname{Aut}_{\mathcal{L}_{1}}\left(S_{0}\right)$ onto $\Gamma$, with kernel $\operatorname{Aut}_{\mathcal{L}_{0}}\left(S_{0}\right)$. Since $S_{0} \in \operatorname{Syl}_{p}\left(\operatorname{Aut}_{\mathcal{L}_{0}}\left(S_{0}\right)\right)$, this shows that $\widehat{\theta}$ induces an isomorphism $S / S_{0} \cong \Gamma$, where $S$ is a Sylow $p$-subgroup 
of $\operatorname{Aut}_{\mathcal{L}_{1}}\left(S_{0}\right)$ which contains $S_{0}$. Hence for any $\psi \in \operatorname{Mor}\left(\mathcal{L}_{1}\right)$, there is $g \in S$ such that $\widehat{\theta}(\psi)=\widehat{\theta}(g)$; and for any such $g$ there is a unique morphism $\psi_{0} \in \operatorname{Mor}\left(\mathcal{L}_{0}\right)$ such that $\psi=\delta(g) \circ \psi_{0}$ where $\delta(g) \in \operatorname{Mor}\left(\mathcal{L}_{1}\right)$ denotes the appropriate restriction of $g \in \operatorname{Aut}_{\mathcal{L}_{1}}\left(S_{0}\right)$. In other words,

$$
\operatorname{Mor}\left(\mathcal{L}_{1}\right) \cong S \times_{S_{0}} \operatorname{Mor}\left(\mathcal{L}_{0}\right)
$$

Step 3: The conjugation action of $S$ on $\operatorname{Mor}\left(\mathcal{L}_{0}\right) \subseteq \operatorname{Mor}\left(\mathcal{L}_{1}\right)$ defines an action of $S$ on $\mathcal{L}_{0}$, which satisfies the hypotheses of Theorem 4.6. (Note in particular that this action sends inclusions to inclusions, since they are assumed to be represented by constant homotopies.) So we can now apply that theorem to construct a $p$-local finite group $(S, \mathcal{F}, \mathcal{L})$ which contains $\left(S_{0}, \mathcal{F}_{0}, \mathcal{L}_{0}\right)$ with $p$-power index. Let $\mathcal{L}^{q}$ be the quasicentric linking system associated to $(S, \mathcal{F}, \mathcal{L})$. By $(4)$, the category $\mathcal{L}_{1}$ defined here is equal to the category $\mathcal{L}_{1}$ defined in the proof of Theorem 4.6 i.e., the full subcategory of $\mathcal{L}^{q}$ with object set $\mathcal{H}_{0}$.

Let $\mathcal{H}$ be the set of subgroups $P \leq S$ such that $P \cap S_{0} \in \mathcal{H}_{0}$, and let $\mathcal{L}_{2} \subseteq \mathcal{L}^{q}$ be the full subcategory with object set $\mathcal{H}$. By Step 4 in the proof of Theorem 4.6, all $\mathcal{F}$-centric $\mathcal{F}$-radical subgroups of $S$ lie in $\mathcal{H}$, and so $|\mathcal{L}| \simeq\left|\mathcal{L}^{q}\right| \simeq\left|\mathcal{L}_{2}\right|$ by Proposition 1.12 (a). Also, $\left|\mathcal{L}_{1}\right|$ is a deformation retract of $\left|\mathcal{L}_{2}\right|$, where the retraction is defined by sending $P \in \mathcal{H}$ to $P \cap S_{0} \in \mathcal{H}_{0}$ (and morphisms are sent to their restrictions, uniquely defined by Proposition $1.12\left(\right.$ b)). Thus $|\mathcal{L}| \simeq\left|\mathcal{L}_{1}\right|$. So by Theorem 4.4, we have a homotopy fibration sequence $\left|\mathcal{L}_{0}\right|_{p}^{\wedge} \rightarrow\left|\mathcal{L}_{1}\right|_{p}^{\wedge} \rightarrow B \Gamma$.

Step 4: It remains to construct a homotopy equivalence $\left|\mathcal{L}_{1}\right| \wedge \longrightarrow X$, which extends to a homotopy equivalence between the fibration sequences. This is where we need to use the topological linking categories defined above. Set

$$
\mathcal{M}_{0}=\mathcal{M}_{S_{0}, f_{0}}^{\mathcal{H}_{0}}\left(X_{0}\right) \quad \text { and } \quad \mathcal{M}_{1}=\mathcal{M}_{S_{0}, f_{0}}^{\mathcal{H}_{0}}(X)
$$

for short, and consider the following commutative diagram:

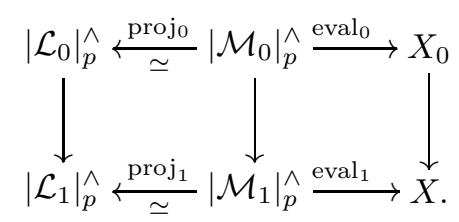

The vertical maps in the diagram are all inclusions. Also, $X_{0}$ is $p$-complete by definition and $X$ by BK, II.5.2(iv)], so the evaluation maps defined above extend to the $p$-completed nerves $\left|\mathcal{M}_{i}\right|_{p}^{\wedge}$. The maps $\operatorname{proj}_{1}$ and eval ${ }_{1}$ both commute up to homotopy with the projections to $B \Gamma$. The projection maps $\operatorname{proj}_{0}$ and $\operatorname{proj}_{1}$ are both homotopy equivalences: the connected components of the morphism spaces in $\mathcal{M}_{i}$ are contractible since the connected components of the fiber and total space in (3) are aspherical (see Step 2).

We claim that eval ${ }_{0}$ is homotopic to $f \circ \operatorname{proj}_{0}$ as maps to $X_{0}$. By naturality, it suffices to check this on the uncompleted nerve $\left|\mathcal{M}_{0}\right|$, and in the case where $X_{0}=|\mathcal{L}|_{p}^{\wedge}$ and $f=\mathrm{Id}$. But in this case, the only real difference between the maps is that eval $l_{0}$ sends all vertices of $\left|\mathcal{M}_{0}\right|$ to the base point of $X_{0}=|\mathcal{L}|_{p}^{\wedge}$, while $\operatorname{proj}_{0}$ sends the vertex for a subgroup $P \leq S$ to the corresponding vertex in $|\mathcal{L}|_{p}^{\wedge}$. So the maps are homotopic via a homotopy which sends vertices to the base point along the edges of $|\mathcal{L}|$ corresponding to the inclusion morphisms. In particular, this shows that eval $_{0}$ is also a homotopy equivalence. We thus have an equivalence between 
the fibration sequences

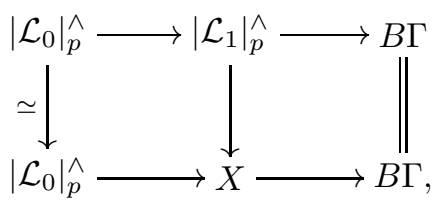

and this proves that the two sequences are equivalent.

Recall (Definition 4.5) that for any saturated fusion system $\mathcal{F}$ over a $p$-group $S$, $\operatorname{Aut}_{\text {fus }}(S, \mathcal{F})$ denotes the group of all fusion preserving automorphisms of $S$. The following corollary to Theorem [4.6 describes how "exotic" fusion systems could potentially arise as extensions of $p$-power index; we still do not know whether the situation it describes can occur.

Corollary 4.8. Fix a finite group $G$, with Sylow subgroup $S \in \operatorname{Syl}_{p}(G)$. Assume there is an automorphism $\bar{\alpha} \in \operatorname{Aut}_{\text {fus }}\left(S, \mathcal{F}_{S}(G)\right)$ of p-power order, which is not the restriction to $S$ of an automorphism of $G$, and which moreover is not the restriction of an automorphism of $G^{\prime}$ for any finite group $G^{\prime}$ with $S \in \operatorname{Syl}_{p}\left(G^{\prime}\right)$ and $\mathcal{F}_{S}\left(G^{\prime}\right)=$ $\mathcal{F}_{S}(G)$. Then there is a saturated fusion system $(\widehat{S}, \widehat{\mathcal{F}}) \supseteq\left(S, \mathcal{F}_{S}(G)\right)$, such that $\mathcal{F}_{S}(G)$ has p-power index in $\widehat{\mathcal{F}}$, and such that $\widehat{\mathcal{F}}$ is not the fusion system of any finite group.

Proof. By [BLO1, Theorem E], together with [01, Theorem A] and [O2, Theorem $\mathrm{A}]$, there is a short exact sequence

$$
0 \longrightarrow{\underset{\mathcal{O}}{\mathcal{O}_{S}^{c}(G)}}^{\lim ^{1}}\left(\mathcal{Z}_{G}\right) \longrightarrow \operatorname{Out}_{\text {typ }}\left(\mathcal{L}_{S}^{c}(G)\right) \longrightarrow \operatorname{Out}_{\text {fus }}\left(S, \mathcal{F}_{S}(G)\right) \longrightarrow 0
$$

where $\operatorname{Out}_{\text {fus }}(S, \mathcal{F})=\operatorname{Aut}_{\text {fus }}(S, \mathcal{F}) / \operatorname{Aut}_{\mathcal{F}}(S)$, where $\mathcal{O}_{S}^{c}(G)$ and $\mathcal{Z}_{G}$ are the category and functor of Definition $1.7(\mathrm{~b})$, and where $\operatorname{Out}_{\text {typ }}\left(\mathcal{L}_{S}^{c}(G)\right)$ is the group of "isotypical" automorphisms of $\mathcal{L}_{S}^{c}(G)$ modulo natural isomorphism (see the introduction of [BLO1], or BLO1, Definition 3.2], for the definition). Let $[\bar{\alpha}]$ be the class of $\bar{\alpha}$ in $\operatorname{Out}_{\text {fus }}\left(S, \mathcal{F}_{S}(G)\right)$; then $[\bar{\alpha}]$ lifts to an automorphism $\alpha$ of the linking system $\mathcal{L}_{S}^{c}(G)$. Upon replacing $\alpha$ by some appropriate power, we can assume that it still has $p$-power order. We can also assume, upon replacing $\alpha$ by another automorphism in the same conjugacy class if necessary, that the $\alpha$-action on $\operatorname{Aut}_{\mathcal{L}}(S)$ leaves invariant the subgroup $\delta_{S}(S)$; i.e., that the action of $\alpha$ on $\mathcal{L}_{S}^{c}(G)$ restricts to an action on $S$.

Set $\widehat{S}=S \rtimes\langle x\rangle$, where $|x|=|\alpha|$ and $x$ acts on $S$ via $\alpha$. Then $\widehat{S}$ has an action on $\mathcal{L}_{S}^{c}(G)$ induced by the actions of $S$ and of $\alpha$, and this action satisfies conditions (a)-(d) in Theorem 4.6. Let $\widehat{\mathcal{F}} \supseteq \mathcal{F}_{S}(G)$ be the saturated fusion system over $\widehat{S}$ constructed by the theorem.

We claim that $\widehat{\mathcal{F}}$ is not the fusion system of any finite group. Assume otherwise: assume $\widehat{\mathcal{F}}$ is the fusion system of a group $\widehat{G}$. Since $\mathcal{F}$ has $p$-power index in $\widehat{\mathcal{F}}$, $S \geq O_{\widehat{\mathcal{F}}}^{p}(\widehat{S})$, and so $S \geq \widehat{S} \cap O^{p}(\widehat{G})$ by the hyperfocal subgroup theorem (Lemma 2.2). Set $G^{\prime}=S \cdot O^{p}(\widehat{G})$. Then $G^{\prime} \triangleleft \widehat{G}$ since $S \triangleleft \widehat{S} ; \widehat{G} / G^{\prime} \cong \widehat{S} / S^{\prime}$, and thus $G^{\prime} \triangleleft \widehat{G}$ has $p$-power index and $S \in \operatorname{Syl}_{p}\left(G^{\prime}\right)$. By Theorem 4.3. there is a unique saturated fusion subsystem over $S$ of $p$-power index in $\widehat{\mathcal{F}}$, and thus $\mathcal{F}=\mathcal{F}_{S}\left(G^{\prime}\right)$. Also, $x \in \widehat{S} \leq \widehat{G}$ acts on $G^{\prime}$ via an automorphism whose restriction to $S$ is $\alpha$, and this contradicts the original assumption about $\bar{\alpha}$. 


\section{Fusion subsystems And EXtensions of IndeX PRIME TO $p$}

In this section, we classify all saturated fusion subsystems of index prime to $p$ in a given saturated fusion system $\mathcal{F}$, and show that there is a unique minimal subsystem $O^{p^{\prime}}(\mathcal{F})$ of this type. More precisely, we show that there is a certain finite group of order prime to $p$ associated to $\mathcal{F}$, denoted below $\Gamma_{p^{\prime}}(\mathcal{F})$, and a one-to-one correspondence between subgroups $T \leq \Gamma_{p^{\prime}}(\mathcal{F})$ and fusion subsystems $\mathcal{F}_{T}$ of index prime to $p$ in $\mathcal{F}$. The index of $\mathcal{F}_{T}$ in $\mathcal{F}$ can then be defined to be the index of $T$ in $\Gamma_{p^{\prime}}(\mathcal{F})$.

Conversely, we also describe extensions of saturated fusion systems of index prime to $p$. Once more the terminology requires motivation. Roughly speaking, an extension of index prime to $p$ of a given saturated fusion system $\mathcal{F}$ is a saturated fusion system $\mathcal{F}^{\prime}$ over the same $p$-group $S$, but where the morphism set has been "extended" by an action of a group of automorphisms whose order is prime to $p$. Here again, a one-to-one correspondence statement is obtained, thus providing a full classification.

As noted in the introduction, Puig Pu1 had earlier showed how to construct the minimal fusion subsystem of index prime to $p$ (which he called the adjoint subsystem), and also showed how to construct extensions of fusion systems of index prime to $p$. What is new here is that we obtain a more explicit classification on the level of fusion systems; and also show how such subsystems and extensions behave on the level of linking systems, in the sense that the geometric realization of the inclusion of linking systems is homotopy equivalent to a covering space projection.

5.1. Subsystems of index prime to $p$. We first classify all saturated fusion subsystems of index prime to $p$ in a given saturated fusion system $\mathcal{F}$. The fusion subsystems and associated linking systems will be constructed using Proposition 3.8 and Theorem 3.9, respectively. More precisely, Theorem 3.9 has already told us that for any $p$-local finite group $(S, \mathcal{F}, \mathcal{L})$, any surjection $\theta$ of $\pi_{1}(|\mathcal{L}|)$ onto a finite $p^{\prime}$-group $\Gamma$, and any subgroup $H \leq \Gamma$, there is a $p$-local finite subgroup $\left(S^{\prime}, \mathcal{F}^{\prime}, \mathcal{L}^{\prime}\right)$ such that $\left|\mathcal{L}^{\prime}\right|$ is homotopy equivalent to the covering space of $|\mathcal{L}|$ with fundamental group $\theta^{-1}(H)$. What is new in this section is first, that we describe the "universal" $p^{\prime}$-group quotient of $\pi_{1}(|\mathcal{L}|)$ as a certain quotient group of $\operatorname{Out}_{\mathcal{F}}(S)$; and second, we show that all fusion subsystems of index prime to $p$ in $\mathcal{F}$ (in the sense of Definition 3.1) are obtained in this way.

When applying Proposition 3.8 to this situation, we need to consider fusion mapping triples $(\Gamma, \theta, \Theta)$ on $\mathcal{F}^{q}$, where $\Gamma$ is finite of order prime to $p$. Since $\theta \in$ $\operatorname{Hom}(S, \Gamma)$, it must then be the trivial homomorphism. In this case, conditions (i)-(iii) in Definition 3.6 are equivalent to requiring that there is some functor $\widehat{\Theta}: \mathcal{F}^{q} \rightarrow \mathcal{B}(\Gamma)$ such that $\Theta(\varphi)=\{\widehat{\Theta}(\varphi)\}$ for each $\varphi \in \operatorname{Mor}\left(\mathcal{F}^{q}\right)$ (and condition (iv) is redundant). So instead of explicitly constructing fusion mapping triples, we instead construct functors of this form.

We start with some definitions. For a finite group $G$, one defines $O^{p^{\prime}}(G)$ to be the smallest normal subgroup of $G$ of index prime to $p$, or equivalently the subgroup generated by elements of $p$-power order in $G$. These two definitions are not, in general, equivalent in the case of an infinite group (the case $G=\mathbb{Z}$ being an obvious example). We do, however, need to deal with such subgroups here. The following definition is most suitable for our purposes, and it is a generalization of the finite case. 
Definition 5.1. For any group $G$ (possibly infinite), let $O^{p^{\prime}}(G)$ be the intersection of all normal subgroups in $G$ of finite index prime to $p$.

In particular, under this definition, an epimorphism $\alpha: G \longrightarrow H$ with $\operatorname{Ker}(\alpha) \leq$ $O^{p^{\prime}}(G)$ induces an isomorphism $G / O^{p^{\prime}}(G) \cong H / O^{p^{\prime}}(H)$. Thus by Proposition 2.6. for any $p$-local finite group $(S, \mathcal{F}, \mathcal{L})$, the projections of $|\mathcal{L}| \simeq\left|\mathcal{L}^{q}\right|$ onto $\left|\mathcal{F}^{c}\right|$ and $\left|\mathcal{F}^{q}\right|$ induce isomorphisms

$$
\pi_{1}(|\mathcal{L}|) / O^{p^{\prime}}\left(\pi_{1}(|\mathcal{L}|)\right) \cong \pi_{1}\left(\left|\mathcal{F}^{c}\right|\right) / O^{p^{\prime}}\left(\pi_{1}\left(\left|\mathcal{F}^{c}\right|\right)\right) \cong \pi_{1}\left(\left|\mathcal{F}^{q}\right|\right) / O^{p^{\prime}}\left(\pi_{1}\left(\left|\mathcal{F}^{q}\right|\right)\right)
$$

Fix a saturated fusion system $\mathcal{F}$ over a $p$-group $S$, and define

$$
\Gamma_{p^{\prime}}(\mathcal{F})=\pi_{1}\left(\left|\mathcal{F}^{c}\right|\right) / O^{p^{\prime}}\left(\pi_{1}\left(\left|\mathcal{F}^{c}\right|\right)\right) \text {. }
$$

We will show that the natural functor

$$
\varepsilon_{\mathcal{F}^{c}}: \mathcal{F}^{c} \longrightarrow \mathcal{B}\left(\Gamma_{p^{\prime}}(\mathcal{F})\right)
$$

induces a bijective correspondence between subgroups of $\Gamma_{p^{\prime}}(\mathcal{F})$ and fusion subsystems of $\mathcal{F}$ of index prime to $p$.

Recall (Definition 3.3) that for any saturated fusion system $\mathcal{F}$ over a $p$-group $S$, $O_{*}^{p^{\prime}}(\mathcal{F}) \subseteq \mathcal{F}$ is the smallest fusion subsystem which contains $O^{p^{\prime}}\left(\operatorname{Aut}_{\mathcal{F}}(P)\right)$ for all $P \leq S$; i.e., the smallest fusion subsystem which contains all automorphisms in $\mathcal{F}$ of $p$-power order. Define

$$
\operatorname{Out}_{\mathcal{F}}^{0}(S)=\left\langle\alpha \in \operatorname{Out}_{\mathcal{F}}(S)|\alpha|_{P} \in \operatorname{Mor}_{O_{*}^{p^{\prime}}(\mathcal{F})}(P, S) \text {, some } \mathcal{F} \text {-centric } P \leq S\right\rangle .
$$

Then $\operatorname{Out}_{\mathcal{F}}^{0}(S) \triangleleft \operatorname{Out}_{\mathcal{F}}(S)$, since $O_{*}^{p^{\prime}}(\mathcal{F})$ is normalized by $\operatorname{Aut}_{\mathcal{F}}(S)($ Lemma $3.4(\mathrm{a}))$.

Proposition 5.2. There is a unique functor

$$
\widehat{\theta}: \mathcal{F}^{c} \longrightarrow \mathcal{B}\left(\operatorname{Out}_{\mathcal{F}}(S) / \operatorname{Out}_{\mathcal{F}}^{0}(S)\right)
$$

with the following properties:

(a) $\widehat{\theta}(\alpha)=\alpha$ (modulo $\left.\operatorname{Out}_{\mathcal{F}}^{0}(S)\right)$ for all $\alpha \in \operatorname{Aut}_{\mathcal{F}}(S)$.

(b) $\hat{\theta}(\varphi)=1$ if $\varphi \in \operatorname{Mor}\left(O_{*}^{p^{\prime}}(\mathcal{F})^{c}\right)$. In particular, $\widehat{\theta}$ sends inclusion morphisms to the identity.

Furthermore, there is an isomorphism

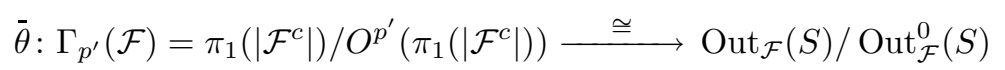

such that $\widehat{\theta}=\mathcal{B} \bar{\theta} \circ \varepsilon_{\mathcal{F}}^{c}$.

Proof. By Lemma 3.4(c), each morphism in $\mathcal{F}^{c}$ factors as the composite of the restriction of a morphism in $\operatorname{Aut}_{\mathcal{F}}(S)$ followed by a morphism in $O_{*}^{p^{\prime}}(\mathcal{F})^{c}$. If

$$
\varphi=\left.\varphi_{1} \circ \alpha_{1}\right|_{P}=\left.\varphi_{2} \circ \alpha_{2}\right|_{P}
$$

where $\varphi \in \operatorname{Hom}_{\mathcal{F}}(P, Q), \alpha_{i} \in \operatorname{Aut}_{\mathcal{F}}(S)$, and $\varphi_{i} \in \operatorname{Hom}_{O_{*}^{p^{\prime}}(\mathcal{F})^{c}}\left(\alpha_{i}(P), Q\right)$, then we can assume (after factoring out inclusions) that all of these are isomorphisms, and hence

$$
\left.\left(\alpha_{2} \circ \alpha_{1}^{-1}\right)\right|_{P}=\varphi_{2}^{-1} \circ \varphi_{1} \in \operatorname{Iso}_{O_{*}^{p^{\prime}}(\mathcal{F})^{c}}\left(\alpha_{1}(P), \alpha_{2}(P)\right) .
$$

Thus $\alpha_{2} \circ \alpha_{1}^{-1} \in \operatorname{Out}_{\mathcal{F}}^{0}(S)$; and so we can define

$$
\widehat{\theta}(\varphi)=\left[\alpha_{1}\right]=\left[\alpha_{2}\right] \in \operatorname{Out}_{\mathcal{F}}(S) / \operatorname{Out}_{\mathcal{F}}^{0}(S) .
$$


This shows that $\widehat{\theta}$ is well defined on morphisms (and sends each object in $\mathcal{F}^{c}$ to the unique object of $\left.\mathcal{B}\left(\operatorname{Out}_{\mathcal{F}}(S) / \operatorname{Out}_{\mathcal{F}}^{0}(S)\right)\right)$. By Lemma 3.4(c) again, $\widehat{\theta}$ preserves compositions, and hence is a well defined functor. It satisfies conditions (a) and (b) above by construction. The uniqueness of $\widehat{\theta}$ is clear.

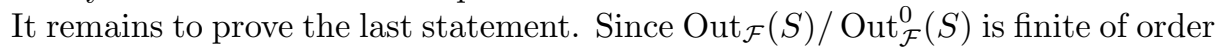
prime to $p, \pi_{1}(|\widehat{\theta}|)$ factors through a homomorphism

$$
\bar{\theta}: \pi_{1}\left(\left|\mathcal{F}^{c}\right|\right) / O^{p^{\prime}}\left(\pi_{1}\left(\left|\mathcal{F}^{c}\right|\right)\right) \longrightarrow \operatorname{Out}_{\mathcal{F}}(S) / \operatorname{Out}_{\mathcal{F}}^{0}(S) .
$$

The inclusion of $B \operatorname{Aut}_{\mathcal{F}}(S)$ into $\left|\mathcal{F}^{c}\right|$ (as the subcomplex with one vertex $S$ ) induces a homomorphism

$$
\tau: \operatorname{Out}_{\mathcal{F}}(S) \longrightarrow \pi_{1}\left(\left|\mathcal{F}^{c}\right|\right) / O^{p^{\prime}}\left(\pi_{1}\left(\left|\mathcal{F}^{c}\right|\right)\right) .
$$

Furthermore, $\tau$ is surjective since $\mathcal{F}=\left\langle O_{*}^{p^{\prime}}(\mathcal{F})\right.$, $\left.\operatorname{Aut}_{\mathcal{F}}(S)\right\rangle$ (Lemma 3.4(b)), and since any automorphism in $O_{*}^{p^{\prime}}(\mathcal{F})$ is a composite of restrictions of automorphisms of $p$-power order. By (a), and since $\theta$ restricted to $\operatorname{Aut}_{\mathcal{F}}(S)$ is the projection onto $\operatorname{Out}_{\mathcal{F}}(S)$, the composite $\bar{\theta} \circ \tau$ is the projection of $\operatorname{Out}_{\mathcal{F}}(S)$ onto the quotient group $\operatorname{Out}_{\mathcal{F}}(S) / \operatorname{Out}_{\mathcal{F}}^{0}(S)$. Finally, $\operatorname{Out}_{\mathcal{F}}^{0}(S) \leq \operatorname{Ker}(\tau)$ by definition of $\operatorname{Out}_{\mathcal{F}}^{0}(S)$, and this shows that $\bar{\theta}$ is an isomorphism.

The following lemma shows that any fusion mapping triple on $\mathcal{F}^{c}$ can be extended uniquely to $\mathcal{F}^{q}$. This will allow us later to apply Proposition 3.8 in order to produce saturated fusion subsystems of index prime to $p$.

Lemma 5.3. Let $\mathcal{F}$ be a saturated fusion system over a p-group $S$, and let $\left(\Gamma, \theta, \Theta_{0}\right)$ be a fusion mapping triple on $\mathcal{F}^{c}$. Then there is a unique extension

$$
\Theta: \operatorname{Mor}\left(\mathcal{F}^{q}\right) \rightarrow \mathfrak{S u b}(\Gamma)
$$

of $\Theta_{0}$, such that $(\Gamma, \theta, \Theta)$ is a fusion mapping triple on $\mathcal{F}^{q}$.

Proof. We construct the extension $\Theta$ one $\mathcal{F}$-conjugacy class at a time. Thus, assume $\Theta$ has been defined on a set $\mathcal{H}_{0}$ of $\mathcal{F}$-quasicentric subgroups of $S$ which is a union of $\mathcal{F}$-conjugacy classes, contains all $\mathcal{F}$-centric subgroups, and is closed under overgroups. Let $\mathcal{P}$ be maximal among $\mathcal{F}$-conjugacy classes of $\mathcal{F}$-quasicentric subgroups not in $\mathcal{H}_{0}$; we show that $\Theta$ can be extended to $\mathcal{H}=\mathcal{H}_{0} \cup \mathcal{P}$.

Fix $P \in \mathcal{P}$ which is fully normalized in $\mathcal{F}$. For each $\alpha \in \operatorname{Aut}_{\mathcal{F}}(P)$, there is an extension $\bar{\alpha} \in \operatorname{Aut}_{\mathcal{F}}\left(P \cdot C_{S}(P)\right)$ (axiom (II)), and we define a map

$$
\Theta_{P}: \operatorname{Aut}_{\mathcal{F}}(P) \rightarrow \mathfrak{S u b}\left(N_{\Gamma}\left(\theta\left(C_{S}(P)\right)\right)\right)
$$

by $\Theta_{P}(\alpha)=\Theta(\bar{\alpha}) \cdot \theta\left(C_{S}(P)\right)$. By Definition 3.6 ((i) and (ii)) $\Theta(\bar{\alpha})$ is a left coset of $\theta\left(C_{S}\left(P \cdot C_{S}(P)\right)\right)$, and by (iv), it is also a right coset (where the right coset representative can be taken to be the same as the one representing the left coset); hence $\Theta_{P}(\alpha)$ is a left and a right coset of $\theta\left(C_{S}(P)\right.$ ) (again with the same coset representative on both sides). If $\bar{\alpha}^{\prime} \in \operatorname{Aut}_{\mathcal{F}}\left(P \cdot C_{S}(P)\right)$ is any other extension of $\alpha$, then by [5A1, Lemma 3.8], there is some $g \in C_{S}(P)$ such that $\bar{\alpha}^{\prime}=c_{g} \circ \bar{\alpha}$. Thus, by Definition 3.6 again, $\Theta\left(\bar{\alpha}^{\prime}\right)=\Theta\left(c_{g} \circ \bar{\alpha}\right)=\theta(g) \Theta(\bar{\alpha})$, and

$\Theta\left(\bar{\alpha}^{\prime}\right) \cdot \theta\left(C_{S}(P)\right)=\theta(g) \Theta(\bar{\alpha}) \cdot \theta\left(C_{S}(P)\right)=\Theta(\bar{\alpha}) \theta(\bar{\alpha}(g)) \cdot \theta\left(C_{S}(P)\right)=\Theta(\bar{\alpha}) \cdot \theta\left(C_{S}(P)\right)$, and so the definition of $\Theta_{P}(\alpha)$ is independent of the choice of the extension $\bar{\alpha}$. This shows that $\Theta_{P}$ is well defined. 
Notice also that $\Theta_{P}$ clearly respects compositions, and since $\Theta_{P}(\alpha)=x$. $\theta\left(C_{S}(P)\right)=\theta\left(C_{S}(P)\right) \cdot x$, for some $x \in \Gamma$, we conclude that $x \in N_{\Gamma}\left(\theta\left(C_{S}(P)\right)\right)$. Thus $\Theta_{P}$ induces a homomorphism

$$
\Theta_{P}: \operatorname{Aut}_{\mathcal{F}}(P) \rightarrow N_{\Gamma}\left(\theta\left(C_{S}(P)\right)\right) / \theta\left(C_{S}(P)\right) .
$$

We now make use of Lemma 4.1, which gives sufficient conditions to the existence of an extension of a fusion mapping triple.

If $\alpha \in \operatorname{Aut}_{\mathcal{F}}(P)$, and $x \in \Theta_{P}(\alpha)$, then $x=y \cdot \theta(h)$ for some $h \in C_{S}(P)$ and $y \in \Theta(\bar{\alpha})$, where $\bar{\alpha}$ is an extension of $\alpha$ to $P \cdot C_{S}(P)$. Hence for any $g \in P$,

$$
x \theta(g) x^{-1}=y \cdot \theta\left(h g h^{-1}\right) y^{-1}=y \theta(g) y^{-1}=\theta(\bar{\alpha}(g))=\theta(\alpha(g)) .
$$

This shows that point $(+)$ of Lemma 4.1 holds, and so it remains to check $(*)$.

Assume $P \supsetneqq Q \leq S, P \triangleleft Q$, and let $\alpha \in \operatorname{Aut}_{\mathcal{F}}(P)$ and $\beta \in \operatorname{Aut}_{\mathcal{F}}(Q)$ be such that $\alpha=\left.\beta\right|_{P}$. Then $Q \cdot C_{S}(P) \leq N_{\alpha}$ in the terminology of axiom (II), so $\alpha$ extends to another automorphism $\gamma \in \operatorname{Aut}_{\mathcal{F}}\left(Q \cdot C_{S}(P)\right)$, and $\Theta_{P}(\alpha)=\Theta(\gamma) \cdot \theta\left(C_{S}(P)\right)$ by definition of $\Theta_{P}$. By [5A1, Lemma 3.8] again, $\left.\gamma\right|_{Q}=c_{g} \circ \beta$ for some $g \in C_{S}(P)$. Hence, by Definition 3.6. $\Theta(\gamma)=\Theta\left(c_{g} \circ \beta\right)=\theta(g) \cdot \Theta(\beta)$, and so

$$
\Theta_{P}(\alpha)=\theta(g) \cdot \Theta(\beta) \cdot \theta\left(C_{S}(P)\right)=\Theta(\beta) \theta(\beta(g)) \cdot \theta\left(C_{S}(P)\right)=\Theta(\beta) \cdot \theta\left(C_{S}(P)\right) .
$$

In particular, $\Theta_{P}(\alpha) \supseteq \Theta(\beta)$. This shows that point $(*)$ of Lemma 4.1 is satisfied as well, and thus, by the lemma, $\Theta$ can be extended to a fusion mapping triple on $\mathcal{F}^{\mathcal{H}}$.

Recall that a fusion subsystem of index prime to $p$ in a saturated fusion system $\mathcal{F}$ over $S$ is a saturated subsystem $\mathcal{F}_{0} \subseteq \mathcal{F}$ over the same $p$-group $S$, such that $\operatorname{Aut}_{\mathcal{F}_{0}}(P) \geq O^{p^{\prime}}\left(\operatorname{Aut}_{\mathcal{F}}(P)\right)$ for all $P \leq \bar{S}$. Equivalently, $\mathcal{F}_{0} \subseteq \mathcal{F}$ has index prime to $p$ if and only if it is saturated and contains the subcategory $O_{*}^{p^{\prime}}(\mathcal{F})$ of Definition 3.3. We are now ready to prove our main result about these subsystems.

Theorem 5.4. For any saturated fusion system $\mathcal{F}$ over a p-group $S$, there is a bijective correspondence between subgroups

$$
H \leq \Gamma_{p^{\prime}}(\mathcal{F})=\operatorname{Out}_{\mathcal{F}}(S) / \operatorname{Out}_{\mathcal{F}}^{0}(S),
$$

and saturated fusion subsystems $\mathcal{F}_{H}$ of $\mathcal{F}$ over $S$ of index prime to $p$ in $\mathcal{F}$. The correspondence is given by associating to $H$ the fusion system generated by $\widehat{\theta}^{-1}(\mathcal{B}(H))$, where $\widehat{\theta}$ is the functor of Proposition 5.2 .

Proof. Let $\mathcal{F}_{0} \subseteq \mathcal{F}$ be any saturated fusion subsystem over $S$ which contains $O_{*}^{p^{\prime}}(\mathcal{F})$. Then $\operatorname{Out}_{\mathcal{F}}^{0}(S) \triangleleft \operatorname{Out}_{\mathcal{F}_{0}}(S)$, and one can set $H=\operatorname{Out}_{\mathcal{F}_{0}}(S) / \operatorname{Out}_{\mathcal{F}}^{0}(S)$. We first show that a morphism $\varphi$ of $\mathcal{F}^{c}$ is in $\mathcal{F}_{0}$ if and only if $\widehat{\theta}(\varphi) \in H$. Clearly it suffices to prove this for isomorphisms in $\mathcal{F}^{c}$.

Let $P, Q \leq S$ be $\mathcal{F}$-centric, $\mathcal{F}$-conjugate subgroups, and fix an isomorphism $\varphi \in \operatorname{Iso}_{\mathcal{F}}(P, Q)$. By Lemma 3.4 we can write $\varphi=\psi \circ\left(\left.\alpha\right|_{P}\right)$, where $\alpha \in \operatorname{Aut}_{\mathcal{F}}(S)$ and $\psi \in \operatorname{Iso}_{O_{*}^{p^{\prime}}(\mathcal{F})}(\alpha(P), Q)$. Then $\varphi$ is in $\mathcal{F}_{0}$ if and only if $\left.\alpha\right|_{P}$ is in $\mathcal{F}_{0}$. Also, by definition of $\widehat{\theta}$ (and of $H$ ), $\widehat{\theta}(\varphi) \in H$ if and only if $\alpha \in \operatorname{Aut}_{\mathcal{F}_{0}}(S)$. So it remains to prove that $\left.\alpha\right|_{P} \in \operatorname{Mor}\left(\mathcal{F}_{0}\right)$ if and only if $\alpha \in \operatorname{Aut}_{\mathcal{F}_{0}}(S)$.

If $\alpha \in \operatorname{Aut}_{\mathcal{F}_{0}}(S)$, then $\left.\alpha\right|_{P}$ is also in $\mathcal{F}_{0}$ by definition of a fusion system. So it remains to prove the converse. Assume $\left.\alpha\right|_{P}$ is in $\mathcal{F}_{0}$. The same argument as that used to prove Proposition 3.8 (c) shows that $\alpha(P)$ is $\mathcal{F}_{0}$-centric, and hence fully centralized in $\mathcal{F}_{0}$. Since $\left.\alpha\right|_{P}$ extends to an (abstract) automorphism of $S$, 
axiom (II) implies that it extends to some $\alpha_{1} \in \operatorname{Hom}_{\mathcal{F}_{0}}\left(N_{S}(P), S\right)$. Since $P$ is $\mathcal{F}$-centric, [BLO2, Proposition A.8] applies to show that $\alpha_{1}=\left(\left.\alpha\right|_{N_{S}(P)}\right) \circ c_{g}$ for some $g \in Z(P)$, and thus that $\left.\alpha\right|_{N_{S}(P)} \in \operatorname{Hom}_{\mathcal{F}_{0}}\left(N_{S}(P), S\right)$. Also, $N_{S}(P) \supsetneqq P$ whenever $P \supsetneqq S$, and so we can continue this process to show that $\alpha \in \operatorname{Aut}_{\mathcal{F}_{0}}(S)$. This finishes the proof that $\mathcal{F}_{0}=\widehat{\theta}^{-1}(H)$.

Now fix a subgroup $H \leq \operatorname{Out}_{\mathcal{F}}(S) / \operatorname{Out}_{\mathcal{F}}^{0}(S)$, and let $\mathcal{F}_{H}$ be the smallest fusion system over $S$ which contains $\widehat{\theta}^{-1}(\mathcal{B}(H))$. We must show that $\mathcal{F}_{H}$ is a saturated fusion subsystem of index prime to $p$ in $\mathcal{F}$. For $\mathcal{F}$-centric subgroups $P, Q \leq S$, $\operatorname{Hom}_{\mathcal{F}_{H}}(P, Q)$ is the set of all morphisms $\varphi$ in $\operatorname{Hom}_{\mathcal{F}}(P, Q)$ such that $\widehat{\theta}(\varphi) \in H$. In particular, $\mathcal{F}_{H} \supseteq O_{*}^{p^{\prime}}(\mathcal{F})$, since morphisms in $O_{*}^{p^{\prime}}(\mathcal{F})$ are sent by $\widehat{\theta}$ to the identity element.

Define $\Theta: \operatorname{Mor}\left(\mathcal{F}^{c}\right) \rightarrow \mathfrak{S u b}\left(\Gamma_{p^{\prime}}(\mathcal{F})\right)$ by setting $\Theta(\varphi)=\{\widehat{\theta}(\varphi)\}$; i.e., the image consists of subsets with one element. Let $\theta \in \operatorname{Hom}\left(S, \Gamma_{p^{\prime}}(\mathcal{F})\right.$ ) be the trivial (and unique) homomorphism. Then $\left(\Gamma_{p^{\prime}}(\mathcal{F}), \theta, \Theta\right)$ is a fusion mapping triple on $\mathcal{F}^{c}$. By Lemma 5.3, this can be extended to a fusion mapping triple on $\mathcal{F}^{q}$; and hence $\mathcal{F}_{H}$ is saturated by Proposition 3.8.

By Theorem 1.5(a) (Alperin's fusion theorem), $\mathcal{F}_{H}$ is the unique saturated fusion subsystem of $\mathcal{F}$ with the property that a morphism $\varphi \in \operatorname{Hom}_{\mathcal{F}}(P, Q)$ between $\mathcal{F}$ centric subgroups of $S$ lies in $\mathcal{F}_{H}$ if and only if $\widehat{\theta}(\varphi) \in H$. This shows that the correspondence is indeed bijective.

The next theorem describes the relationship between subgroups of index prime to $p$ in a $p$-local finite group $(S, \mathcal{F}, \mathcal{L})$ and certain covering spaces of $|\mathcal{L}|$.

Theorem 5.5. Fix a p-local finite group $(S, \mathcal{F}, \mathcal{L})$. Then for each subgroup $H \leq$ $\operatorname{Out}_{\mathcal{F}}(S)$ containing $\operatorname{Out}_{\mathcal{F}}^{0}(S)$, there is a unique p-local finite subgroup $\left(S, \mathcal{F}_{H}, \mathcal{L}_{H}\right)$, such that $\mathcal{F}_{H}$ has index prime to $p$ in $\mathcal{F}$, $\operatorname{Out}_{\mathcal{F}_{H}}(S)=H$, and $\mathcal{L}_{H}=\pi^{-1}\left(\mathcal{F}_{H}\right)$ (where $\pi$ is the usual functor from $\mathcal{L}$ to $\mathcal{F}$ ). Furthermore, $\left|\mathcal{L}_{H}\right|$ is homotopy equivalent, via its inclusion into $|\mathcal{L}|$, to the covering space of $|\mathcal{L}|$ with fundamental group $\widetilde{H}$ : where $\widetilde{H} \leq \pi_{1}(|\mathcal{L}|)$ is the subgroup such that $\bar{\theta}\left(\widetilde{H} / O^{p^{\prime}}\left(\pi_{1}(|\mathcal{L}|)\right)\right)$ corresponds to $H / \operatorname{Out}_{\mathcal{F}}^{0}(S)$ under the isomorphism

$$
\pi_{1}(|\mathcal{L}|) / O^{p^{\prime}}\left(\pi_{1}(|\mathcal{L}|)\right) \cong \pi_{1}\left(\left|\mathcal{F}^{c}\right|\right) / O^{p^{\prime}}\left(\pi_{1}\left(\left|\mathcal{F}^{c}\right|\right)\right) \stackrel{\bar{\theta}}{\cong} \operatorname{Out}_{\mathcal{F}}(S) / \operatorname{Out}_{\mathcal{F}}^{0}(S)
$$

of Proposition 5.2.

Proof. By Theorem 3.9, applied to the composite functor

$$
\mathcal{L} \stackrel{\pi}{\longrightarrow} \mathcal{F}^{c} \stackrel{\widehat{\theta}}{\longrightarrow} \mathcal{B}\left(\operatorname{Out}_{\mathcal{F}}(S) / \operatorname{Out}_{\mathcal{F}}^{0}(S)\right),
$$

$\left(S, \mathcal{F}_{H}, \mathcal{L}_{H}\right)$ is a $p$-local finite group, and $\left|\mathcal{L}_{H}\right|$ is homotopy equivalent to the covering space of $|\mathcal{L}|$ with fundamental group $\widetilde{H}$ defined above. The uniqueness follows from Theorem [5.4.

Theorem 5.4 shows, in particular, that any saturated fusion system $\mathcal{F}$ over $S$ contains a unique minimal saturated subsystem $O^{p^{\prime}}(\mathcal{F})$ of index prime to $p$ : the subsystem $\mathcal{F}_{0} \subseteq \mathcal{F}$ with $\operatorname{Out}_{\mathcal{F}_{0}}(S)=\operatorname{Out}_{\mathcal{F}}^{0}(S)$. Furthermore, if $\mathcal{F}$ has an associated centric linking system $\mathcal{L}$, then Theorem $\left[5.5\right.$ shows that $O^{p^{\prime}}(\mathcal{F})$ has an associated linking system $O^{p^{\prime}}(\mathcal{L})$, whose geometric realization $\left|O^{p^{\prime}}(\mathcal{L})\right|$ is homotopy equivalent to the covering space of $|\mathcal{L}|$ with fundamental group $O^{p^{\prime}}\left(\pi_{1}(|\mathcal{L}|)\right)$. We emphasize, 
however, that if $\mathcal{F}$ is the fusion system of a finite group $G$, then $O^{p^{\prime}}(\mathcal{F})$ need not be the fusion system of $O^{p^{\prime}}(G): G=A_{5}$ (with $p=2$ ) provides an easy counterexample.

Remark added in proof. Michael Aschbacher has pointed out to us that for any saturated fusion system $\mathcal{F}$ over a $p$-group $S, \pi_{1}\left(\left|\mathcal{F}^{c}\right|\right)$ is finite of order prime to $p$, and hence is isomorphic to the group $\operatorname{Out}_{\mathcal{F}}(S) / \operatorname{Out}_{\mathcal{F}}^{0}(S)$ which appears in Theorems 5.4 and 5.5. In other words, there is a bijective correspondence between the set of saturated fusion subsystems of index prime to $p$ in $\mathcal{F}$ and the set of subgroups of $\pi_{1}\left(\left|\mathcal{F}^{c}\right|\right)$, or equivalently the set of covering spaces of $\left|\mathcal{F}^{c}\right|$. This result can be deduced from Lemma 3.4 and Proposition 5.2, using that the elements of $\operatorname{Inn}(S)$ (as a subgroup of $\operatorname{Aut}_{\mathcal{F}}(S)$ ) represent the identity in $\pi_{1}\left(\left|\mathcal{F}^{c}\right|\right)$.

5.2. Extensions of index prime to $p$. It remains to consider the opposite problem: that of describing the extensions of a given saturated fusion system of index prime to $p$. As before, for a saturated fusion system $\mathcal{F}$ over a $p$-group $S$, $\operatorname{Aut}_{\text {fus }}(S, \mathcal{F})$ denotes the group of fusion preserving automorphisms of $S$ (Definition 4.5). Theorem 5.7 below states that each subgroup of $\operatorname{Out}_{\text {fus }}(S, \mathcal{F})=$ $\operatorname{Aut}_{\text {fus }}(S, \mathcal{F}) / \operatorname{Aut}_{\mathcal{F}}(S)$ of order prime to $p$ gives rise to an extension of $\mathcal{F}$.

The following lemma will be needed to compare the obstructions to the existence and uniqueness of linking systems, in a fusion system and in a fusion subsystem of index prime to $p$. Recall the definition of the orbit category of a fusion system in Definition 1.7.

Lemma 5.6. Fix a saturated fusion system $\mathcal{F}$ over a p-group $S$, and let $\mathcal{F}^{\prime} \subseteq \mathcal{F}$ be a saturated subsystem of index prime to $p$. Assume $\operatorname{Out}_{\mathcal{F}^{\prime}}(S) \triangleleft \operatorname{Out}_{\mathcal{F}}(S)$, and set $\pi=\operatorname{Out}_{\mathcal{F}}(S) / \operatorname{Out}_{\mathcal{F}^{\prime}}(S)$. Then for any $F: \mathcal{O}^{c}(\mathcal{F}) \rightarrow \mathbb{Z}_{(p)}$-mod, there is a natural action of $\pi$ on the higher limits of $\left.F\right|_{\mathcal{O}^{c}\left(\mathcal{F}^{\prime}\right)}$, and

$$
\lim _{\mathcal{O}^{c}(\mathcal{F})}^{*}(F) \cong\left[{\underset{\mathcal{O}^{c}\left(\mathcal{F}^{\prime}\right)}{ }}^{*}\left(\left.F\right|_{\mathcal{O}^{c}\left(\mathcal{F}^{\prime}\right)}\right)\right]^{\pi}
$$

Proof. Let $\mathcal{O}^{c}(\mathcal{F})$-mod denote the category of functors $\mathcal{O}^{c}(\mathcal{F})^{\mathrm{op}} \rightarrow$ Ab. For any $F$ in $\mathcal{O}^{c}(\mathcal{F})$-mod, $\operatorname{Out}_{\mathcal{F}}(S)$ acts on $\prod_{P \in \operatorname{Ob}\left(\mathcal{O}^{c}(\mathcal{F})\right)} F(P)$ by letting $\alpha \in \operatorname{Out}_{\mathcal{F}}(S)$ send $F(\alpha(P))$ to $F(P)$ via the induced map $\alpha^{*}$. This restricts to an action of $\pi$ on $\lambda(F) \stackrel{\text { def }}{=} \varliminf_{\mathcal{O}^{c}\left(\mathcal{F}^{\prime}\right)}\left(\left.F\right|_{\mathcal{O}^{c}\left(\mathcal{F}^{\prime}\right)}\right)$; and by definition of inverse limits,

$$
\lim _{\mathcal{O}^{c}(\mathcal{F})}(F) \cong\left[\varliminf_{\mathcal{O}^{c}\left(\mathcal{F}^{\prime}\right)}\left(\left.F\right|_{\mathcal{O}^{c}\left(\mathcal{F}^{\prime}\right)}\right)\right]^{\pi}=[\lambda(F)]^{\pi}
$$

Since $\mathcal{F}^{\prime}$ is a subsystem of index prime to $p$, $\operatorname{Out}_{\mathcal{F}^{\prime}}(S)$ is a subgroup of $\operatorname{Out}_{\mathcal{F}}^{0}(S)$. Hence, by a slight abuse of notation, one has a functor $\widehat{\theta}: \mathcal{F} \rightarrow \mathcal{B}(\pi)$ given as the composite of the functor $\widehat{\theta}$ of Proposition $[5.2$ with projection to $\pi$. For any $\mathbb{Z}[\pi]$ module $M$, regarded as a functor on $\mathcal{B}(\pi)$, we let $\beta(M)$ denote the composite functor $M \circ \widehat{\theta}$. Then $\mathbb{Z}[\pi]-\bmod \rightarrow^{\beta} \mathcal{O}^{c}(\mathcal{F})$-mod is an exact functor, and a left adjoint to $\mathcal{O}^{c}(\mathcal{F})-\bmod \rightarrow^{\lambda} \mathbb{Z}[\pi]$-mod. In particular, the existence of a left adjoint shows that $\lambda$ sends injective objects in $\mathcal{O}^{c}(\mathcal{F})$-mod to injective $\mathbb{Z}[\pi]$-modules. 
Thus, if $0 \rightarrow F \rightarrow I_{0} \rightarrow I_{1} \rightarrow \cdots$ is an injective resolution of $F$ in $\mathcal{O}^{c}(\mathcal{F})$-mod, and $F$ takes values in $\mathbb{Z}_{(p)}$-modules, then

$$
\begin{aligned}
\lim _{\mathcal{O}^{c}(\mathcal{F})}^{*}(F) & \cong H^{*}\left(0 \rightarrow \lambda\left(I_{0}\right)_{(p)}^{\pi} \rightarrow \lambda\left(I_{1}\right)_{(p)}^{\pi} \rightarrow \cdots\right) \\
& \cong\left[H^{*}\left(0 \rightarrow \lambda\left(I_{0}\right)_{(p)} \rightarrow \lambda\left(I_{1}\right)_{(p)} \rightarrow \cdots\right)\right]^{\pi} \\
& \cong[\underbrace{}_{\mathcal{O}^{c}\left(\mathcal{F}^{\prime}\right)} \lim ^{*}\left(\left.F\right|_{\mathcal{O}^{c}\left(\mathcal{F}^{\prime}\right)}\right)]^{\pi} .
\end{aligned}
$$

We are now ready to examine extensions of index prime to $p$.

Theorem 5.7. Fix a saturated fusion system $\mathcal{F}$ over a p-group $S$. Let

$$
\pi \leq \operatorname{Out}_{\text {fus }}(S, \mathcal{F}) \stackrel{\text { def }}{=} \operatorname{Aut}_{\text {fus }}(S, \mathcal{F}) / \operatorname{Aut}_{\mathcal{F}}(S)
$$

be any subgroup of order prime to $p$, and let $\widetilde{\pi}$ denote the inverse image of $\pi$ in $\operatorname{Aut}_{\text {fus }}(S, \mathcal{F})$.

(a) Let $\mathcal{F} . \pi$ be the fusion system over $S$ generated (as a category) by $\mathcal{F}$ together with restrictions of automorphisms in $\tilde{\pi}$. Then $\mathcal{F} . \pi$ is a saturated fusion system, which contains $\mathcal{F}$ as a fusion subsystem of index prime to $p$.

(b) If $\mathcal{F}$ has an associated centric linking system, then so does $\mathcal{F}$. $\pi$.

(c) Let $\mathcal{L}$ be a centric linking system associated to $\mathcal{F}$. Assume that for each $\alpha \in \pi$, the action of $\alpha$ on $\mathcal{F}$ lifts to an action on $\mathcal{L}$. Then there is a unique centric linking system $\mathcal{L} . \pi$ associated to $\mathcal{F}$. $\pi$ whose restriction to $\mathcal{F}$ is $\mathcal{L}$.

Proof. (a) By definition, every morphism in $\mathcal{F} . \pi$ is the composite of morphisms in $\mathcal{F}$ and restrictions of automorphisms of $S$ which normalize $\mathcal{F}$. If $\psi \in \operatorname{Hom}_{\mathcal{F}}(P, Q)$ and $\varphi \in \operatorname{Aut}_{\text {fus }}(S, \mathcal{F})$, then one has

$$
\left.\varphi\right|_{Q} \circ \psi=\left.\left.\left.\varphi\right|_{Q} \circ \psi \circ \varphi^{-1}\right|_{\varphi(P)} \circ \varphi\right|_{P}=\left.\psi^{\prime} \circ \varphi\right|_{P},
$$

where $\psi^{\prime} \in \operatorname{Hom}_{\mathcal{F}}(\varphi(P), \varphi(Q))$ (since $\varphi$ is fusion preserving). Hence each morphism in $\mathcal{F} . \pi$ is the composite of the restriction of a morphism in $\widetilde{\pi}$ followed by a morphism in $\mathcal{F}$.

We next claim $\mathcal{F}$ is a fusion subsystem of $\mathcal{F} . \pi$ of index prime to $p$. More precisely, we will show, for all $P \leq S$, that $\operatorname{Aut}_{\mathcal{F}}(P) \triangleleft \operatorname{Aut}_{\mathcal{F} . \pi}(P)$ with index prime to $p$. To see this, let $\pi_{1} \subseteq \tilde{\pi}$ be the set of automorphisms $\varphi$ in $\tilde{\pi} \leq \operatorname{Aut}_{\text {fus }}(S, \mathcal{F})$ such that $\varphi(P)$ is $\mathcal{F}$-conjugate to $P$, and let $\pi_{0} \subseteq \pi_{1}$ be the set of classes $\varphi$ such that $\left.\varphi\right|_{P} \in \operatorname{Hom}_{\mathcal{F}}(P, S)$. If $\varphi(P)$ and $\psi(P)$ are both $\mathcal{F}$-conjugate to $P$, then $\psi(\varphi(P))$ is $\mathcal{F}$-conjugate to $\psi(P)$ since $\psi$ is fusion preserving, and thus is $\mathcal{F}$-conjugate to $P$. This shows that $\pi_{1}$ is a subgroup of $\tilde{\pi}$, and an argument using (1) shows that $\pi_{0}$ is also a subgroup. By definition, $\pi_{0} \geq \operatorname{Aut}_{\mathcal{F}}(S)$, so $\pi_{1} / \pi_{0}$ has order prime to $p$ since $\pi$ does. Using (1), define

$$
\theta_{P}: \operatorname{Aut}_{\mathcal{F} . \pi}(P) \longrightarrow \pi_{1} / \pi_{0}
$$

by setting $\theta_{P}(\alpha)=[\psi]$ if $\alpha=\left.\psi\right|_{\beta(P)} \circ \beta$ for some $\beta \in \operatorname{Hom}_{\mathcal{F}}(P, S)$ and some $\psi \cdot \operatorname{Aut}_{\mathcal{F}}(S) \in \pi$. By definition of $\pi_{0}$, this is well defined, and $\operatorname{Ker}\left(\theta_{P}\right)=\operatorname{Aut}_{\mathcal{F}}(P)$. Since $\pi_{1} / \pi_{0}$ has order prime to $p$, this shows that $\operatorname{Aut}_{\mathcal{F}}(P) \triangleleft \operatorname{Aut}_{\mathcal{F} . \pi}(P)$ with index prime to $p$.

We next claim that $\mathcal{F}$ and $\mathcal{F}$. $\pi$ have the same fully centralized, fully normalized and centric subgroups (compare with the proof of Proposition 3.8(c)). By definition, $\mathcal{F}$ and $\mathcal{F}$. $\pi$ are fusion systems over the same $p$-group $S$. Since each $\mathcal{F}$-conjugacy 
class is contained in some $\mathcal{F}$. $\pi$-conjugacy class, any subgroup $P \leq S$ which is fully centralized (fully normalized) in $\mathcal{F} . \pi$ is fully centralized (fully normalized) in $\mathcal{F}$. By the same argument, any $\mathcal{F}$. $\pi$-centric subgroup is also $\mathcal{F}$-centric.

Conversely, assume that $P$ is not fully centralized in $\mathcal{F}$. $\pi$, and let $P^{\prime} \leq S$ be a subgroup $\mathcal{F}$. $\pi$-conjugate to $P$ such that $\left|C_{S}\left(P^{\prime}\right)\right|>\left|C_{S}(P)\right|$. Let $\psi: P^{\prime} \rightarrow P$ be an $\mathcal{F}$. $\pi$-isomorphism between them. Then, by the argument above $\psi=\psi^{\prime} \circ \varphi$, where $\psi^{\prime}$ is a morphism in $\mathcal{F}$ and $\varphi \in \tilde{\pi}$ is the restriction to $P^{\prime}$ of an automorphism of $S$. Hence $\left|C_{S}\left(\varphi\left(P^{\prime}\right)\right)\right|=\left|C_{S}\left(P^{\prime}\right)\right|>\left|C_{S}(P)\right|$, and since $\varphi\left(P^{\prime}\right)$ is $\mathcal{F}$-conjugate to $P$, this shows that $P$ is not fully centralized in $\mathcal{F}$. A similar argument shows that if $P$ is not fully normalized (or not centric) in $\mathcal{F}$. $\pi$, then it is not fully normalized (or not centric) in $\mathcal{F}$.

We prove that $\mathcal{F} . \pi$ is saturated using Theorem 1.5(b). Thus, we must show that conditions (I) and (II) of Definition 1.2 are satisfied for all $\mathcal{F}$. $\pi$-centric subgroups, and that $\mathcal{F} . \pi$ is generated by restrictions of morphisms between its centric subgroups. Let $P \leq S$ be a subgroup which is fully normalized in $\mathcal{F}$. $\pi$. Then it is fully normalized in $\mathcal{F}$, and since $\mathcal{F}$ is saturated, it is fully centralized there and $\operatorname{Aut}_{S}(P) \in \operatorname{Syl}_{p}\left(\operatorname{Aut}_{\mathcal{F}}(P)\right)=\operatorname{Syl}_{p}\left(\operatorname{Aut}_{\mathcal{F} . \pi}\right)$. Hence, condition (I) holds for any $P$ in $(\mathcal{F} \cdot \pi)^{c}$.

Let $\psi: P \rightarrow Q$ be a morphism in $\mathcal{F}$. $\pi$, and write $\psi=\psi^{\prime} \circ \varphi$, as before. Set

$$
N_{\psi}=\left\{g \in N_{S}(P) \mid \psi \circ c_{g} \circ \psi^{-1} \in \operatorname{Aut}_{S}(\psi(P))\right\} ;
$$

then $\varphi\left(N_{\psi}\right)=N_{\psi^{\prime}}$ since $\varphi c_{g} \varphi^{-1}=c_{\varphi(g)}$ for all $g \in S$. Since condition (II) holds for $\mathcal{F}$, the morphism $\psi^{\prime}$ can be extended to $N_{\psi^{\prime}}$. Hence $\psi=\psi^{\prime} \circ \varphi$ can be extended to $N_{\psi}=\varphi\left(N_{\psi^{\prime}}\right)$, and so condition (II) holds for $\mathcal{F} . \pi$.

That all morphisms in $\mathcal{F} . \pi$ are composites of restrictions of $\mathcal{F}$. $\pi$-morphisms between $\mathcal{F}$. $\pi$-centric subgroups holds by construction. Thus Theorem 1.5(b) applies, and $\mathcal{F}$. $\pi$ is saturated.

(b) Let $\mathcal{Z}_{\mathcal{F}}: \mathcal{O}^{c}(\mathcal{F}) \rightarrow A$ b be the functor $\mathcal{Z}_{\mathcal{F}}(P)=Z(P)$, and similarly for $\mathcal{Z}_{\mathcal{F} . \pi}$ (see Definition 1.7). By Lemma 5.6, restriction of categories induces a monomorphism

$$
\underset{\mathcal{O}^{c}(\mathcal{F} . \pi)}{\lim ^{*}}\left(\mathcal{Z}_{\mathcal{F} . \pi}\right) \longrightarrow \lim _{\mathcal{O}^{c}(\mathcal{F})}^{*}\left(\mathcal{Z}_{\mathcal{F}}\right)
$$

whose image is the subgroup of $\pi$-invariant elements.

By [BLO2, Proposition 3.1], the obstruction $\eta(\mathcal{F} . \pi)$ to the existence of a centric linking system associated to $\mathcal{F} . \pi$ lies in $\lim ^{3}\left(\mathcal{Z}_{\mathcal{F} . \pi}\right)$. From the construction in BLO2 of these obstructions (and the fact that $\mathcal{F} . \pi$ and $\mathcal{F}$ have the same centric subgroups), it is clear that the restriction map $(2)$ sends $\eta(\mathcal{F} . \pi)$ to $\eta(\mathcal{F})$. So if there is a linking system $\mathcal{L}$ associated to $\mathcal{F}$, then $\eta(\mathcal{F})=0$, so $\eta(\mathcal{F} . \pi)=0$ by Lemma 5.6. and there is a linking system $\mathcal{L}$. $\pi$ associated to $\mathcal{F} . \pi$.

(c) Let $\mathcal{L}$. $\pi$ be a centric linking system associated to $\mathcal{F}$. $\pi$, as constructed in (b), and let $\mathcal{L}^{\prime}$ be its restriction to $\mathcal{F}$ (i.e., the inverse image of $\mathcal{F}$ under the the projection $\left.\mathcal{L} . \pi \rightarrow(\mathcal{F} . \pi)^{c}\right)$. By BLO2, Proposition 3.1] again, the group $\lim ^{2}\left(\mathcal{Z}_{\mathcal{F}}\right)$ acts freely and transitively on the set of all centric linking systems associated to $\mathcal{F}$. If the action on $\mathcal{F}$ of each $\alpha \in \pi$ lifts to an action on $\mathcal{L}$, then the element of $\lim ^{2}\left(\mathcal{Z}_{\mathcal{F}}\right)$ which measures the difference between $\mathcal{L}$ and $\mathcal{L}^{\prime}$ is $\pi$-invariant, and hence (by Lemma 5.6 again) is the restriction of an element of $\varliminf^{2}\left(\mathcal{Z}_{\mathcal{F} . \pi}\right)$. Upon modifying the equivalence class of $\mathcal{L} . \pi$ by this element, if necessary, we get a centric linking system associated to $\mathcal{F}$. $\pi$ whose restriction to $\mathcal{F}$ is $\mathcal{L}$. 
As was done in the last section for extensions with a $p$-group quotient, we now translate this last result to a theorem stated in terms of fibration sequences.

Theorem 5.8. Fix a p-local finite group $(S, \mathcal{F}, \mathcal{L})$, a finite group $\Gamma$ of order prime to $p$, and a fibration $E \stackrel{v}{\longrightarrow} B \Gamma$ with fiber homotopy equivalent to $|\mathcal{L}|_{p}^{\wedge}$. Then there is a p-local finite group $\left(S, \mathcal{F}^{\prime}, \mathcal{L}^{\prime}\right)$ such that $\mathcal{F} \subseteq \mathcal{F}^{\prime}$ is normal of index prime to $p$, $\operatorname{Aut}_{\mathcal{F}^{\prime}}(S) / \operatorname{Aut}_{\mathcal{F}}(S)$ is a quotient group of $\Gamma$, and $E_{p}^{\wedge} \simeq\left|\mathcal{L}^{\prime}\right|_{p}^{\wedge}$.

Proof. For any space $Y$, let $\operatorname{Aut}(Y)$ denote the topological monoid of homotopy equivalences $Y \stackrel{\simeq}{\longrightarrow} Y$. Fibrations with fiber $Y$ and base $B$ are classified by homotopy classes of maps $B \rightarrow B \operatorname{Aut}(Y)$. This follows, for example, as a special case of the main theorem in $[\mathrm{DKS}]$.

We are thus interested in the classifying space $B \operatorname{Aut}\left(|\mathcal{L}|_{p}^{\wedge}\right)$, whose homotopy groups were determined in [BLO2, §8]. To describe these, let $\operatorname{Aut}_{\text {typ }}(\mathcal{L})$ be the monoid of isotypical self equivalences of the category $\mathcal{L}$; i.e., the monoid of all equivalences of categories $\psi \in \operatorname{Aut}(\mathcal{L})$ such that for all $P \in \mathrm{Ob}(\mathcal{L}), \psi_{P, P}\left(\operatorname{Im}\left(\delta_{P}\right)\right)=$ $\operatorname{Im}\left(\delta_{\psi(P)}\right)$. Let $\mathrm{Out}_{\text {typ }}(\mathcal{L})$ be the group of all isotypical self equivalences modulo natural isomorphisms of functors. By $\left[\mathrm{BLO} 2\right.$, Theorem 8.1], $\pi_{i}\left(B \operatorname{Aut}\left(|\mathcal{L}|_{p}^{\wedge}\right)\right)$ is a finite $p$-group for $i=2$ and vanishes for $i>2$, and

$$
\pi_{1}\left(B \operatorname{Aut}\left(|\mathcal{L}|_{p}^{\wedge}\right)\right) \cong \operatorname{Out}_{\text {typ }}(\mathcal{L})
$$

Each isotypical self equivalence of $\mathcal{L}$ is naturally isomorphic to one which sends inclusions to inclusions (this was shown in [BLO1, Lemma 5.1] for linking systems of a group, and the general case follows by the same argument). Thus each element of $\operatorname{Out}_{\text {typ }}(\mathcal{L})$ is represented by some $\beta$ which sends inclusions to inclusions. This in turn implies that $\beta_{S}$ - the restriction of $\beta_{S, S}$ to $\delta_{S}(S) \leq \operatorname{Aut}_{\mathcal{L}}(S)$ - lies in $\operatorname{Aut}_{\text {fus }}(S, \mathcal{F})$, and that for every $\mathcal{F}$-centric subgroup $P$ of $S$, the functor $\beta$ sends $P$ to the subgroup $\beta_{S}(P) \leq S$. In particular, $\beta$ is an automorphism of $\mathcal{L}$, since it induces a bijection on the set of objects.

Now let $B \Gamma \rightarrow^{f_{v}} B$ Aut $\left(|\mathcal{L}|_{p}^{\wedge}\right)$ be the map which classifies the fibration $E \stackrel{v}{\rightarrow} B \Gamma$. Since $\Gamma$ is a $p^{\prime}$-group, the fibration $E \stackrel{v}{\longrightarrow} B \Gamma$ is uniquely determined by the action of $\Gamma$ on $|\mathcal{L}|_{p}^{\wedge}$; more precisely, by the map induced by $f_{v}$ on fundamental groups:

$$
\pi_{1}\left(f_{v}\right): \Gamma \longrightarrow \operatorname{Out}_{\text {typ }}(\mathcal{L})
$$

By an argument identical to that used to prove [BLO1, Theorem 6.2], there is an exact sequence

$$
0 \longrightarrow \lim _{\mathcal{O}^{c}(\mathcal{F})}^{1}\left(\mathcal{Z}_{\mathcal{F}}\right) \longrightarrow \operatorname{Out}_{\text {typ }}(\mathcal{L}) \stackrel{\mu_{\mathcal{L}}}{\longrightarrow} \operatorname{Out}_{\text {fus }}(S, \mathcal{F})
$$

where $\mu_{\mathcal{L}}$ is defined by restricting a functor $\mathcal{L} \rightarrow \mathcal{L}$ to $\delta_{S}(S) \leq \operatorname{Aut}_{\mathcal{L}}(S)$. Also, $\mathcal{O}^{c}(\mathcal{F})$ and $\mathcal{Z}_{\mathcal{F}}$ are as in Definition [1.7. but all that we need to know here is that $\operatorname{Ker}\left(\mu_{\mathcal{L}}\right)$ is a $p$-group. Set

$$
\psi_{v}=\mu_{\mathcal{L}} \circ \pi_{1}\left(f_{v}\right): \Gamma \longrightarrow \operatorname{Out}_{\text {fus }}(S, \mathcal{F}) .
$$

Set $\pi=\operatorname{Im}\left(\psi_{v}\right) \leq \operatorname{Out}_{\text {fus }}(S, \mathcal{F})$. By its definition, $\psi_{v}$ comes equipped with a lift to $\operatorname{Out}_{\text {typ }}(\mathcal{L})$. So by the above remarks, every element of $\pi$ lifts to an automorphism of $\mathcal{L}$. Let $(S, \mathcal{F} . \pi, \mathcal{L} . \pi)$ be the $p$-local finite group constructed in Theorem 5.7(a), (c) as an extension of $(S, \mathcal{F}, \mathcal{L})$. This induces a fibration

$$
|\mathcal{L}| \longrightarrow|\mathcal{L} . \pi| \longrightarrow B \pi \text {. }
$$


By [BK, Corollary I.8.3], there is a fiberwise completion $E_{w}$ of $|\mathcal{L} . \pi|$ which sits in a fibration sequence

$$
|\mathcal{L}|_{p}^{\wedge} \longrightarrow E_{w} \stackrel{w}{\longrightarrow} B \pi
$$

and this fibration is classified by a map

$$
f_{w}: B \pi \longrightarrow B \operatorname{Aut}\left(|\mathcal{L}|_{p}^{\wedge}\right) .
$$

As was the case for $f_{v}$, the induced map between fundamental groups $\pi_{1}\left(f_{w}\right)$ determines the fibration.

Consider the diagram

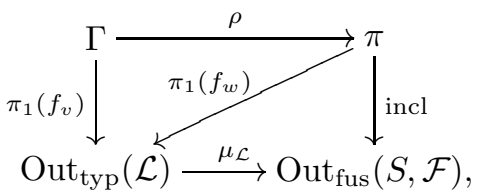

where $\rho: \Gamma \rightarrow \pi$ is the restriction of $\psi_{v}$ to its image, and where the square commutes since each composite is equal to $\psi_{v}$. The composite $\mu_{\mathcal{L}} \circ \pi_{1}\left(f_{w}\right) \in$ $\operatorname{Hom}\left(\pi, \operatorname{Out}_{\text {fus }}(S, \mathcal{F})\right)$ is the homomorphism induced by the homotopy action of $\pi=\pi_{1}(B \pi)$ on the fiber $|\mathcal{L}|_{p}^{\wedge}$. By the construction in Theorem [5.7, this is just the inclusion map. Hence the lower right triangle in the above diagram commutes. Since $\Gamma$ has order prime to $p$ and $\operatorname{Ker}\left(\mu_{\mathcal{L}}\right) \cong \lim ^{1}\left(\mathcal{Z}_{\mathcal{F}}\right)$ is a $p$-group, any homomorphism from $\Gamma$ to $\operatorname{Out}_{\text {fus }}(S, \mathcal{F})$ has a unique conjugacy class of lifting to $\operatorname{Out}_{\text {typ }}(\mathcal{L})$ by the Schur-Zassenhaus theorem (cf. Go, Theorem 6.2.1]). In particular, $\pi_{1}\left(f_{w}\right) \circ \rho$ and $\pi_{1}\left(f_{v}\right)$ are conjugate as homomorphisms from $\Gamma$ to $\operatorname{Out}_{\text {typ }}(\mathcal{L})$. Thus, the upper left triangle in (1) commutes up to conjugacy.

Since $\pi_{2}\left(B \operatorname{Aut}\left(|\mathcal{L}|_{p}^{\wedge}\right)\right)$ is a finite $p$-group and the higher homotopy groups all vanish, we have shown that $f_{w} \circ B \rho$ is homotopic to $f_{v}$. In other words, we have a map of fibrations

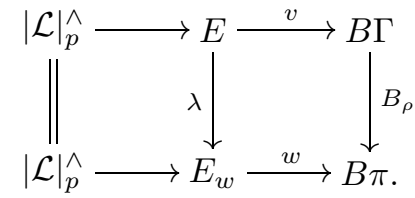

A comparison of the spectral sequences for these two fibrations shows that $\lambda$ is an $\mathbb{F}_{p}$-homology equivalence. Since $|\mathcal{L}|$ is $p$-good [BLO2, Proposition 1.12], the natural map from $|\mathcal{L} . \pi|$ to its fiberwise completion $E_{w}$ is also an $\mathbb{F}_{p}$-homology equivalence. Hence these maps induce homotopy equivalences $E_{p}^{\wedge} \simeq\left(E_{w}\right)_{p}^{\wedge} \simeq|\mathcal{L} . \pi|_{p}^{\wedge}$. This finishes the proof of the theorem, with $\mathcal{F}^{\prime}=\mathcal{F} . \pi$ and $\mathcal{L}^{\prime}=\mathcal{L}$. $\pi$.

Note that we are not assuming that $\Gamma$ injects into $\operatorname{Out}_{\text {fus }}(S, \mathcal{F})$ in the hypotheses of Theorem 5.8. Thus, for example, if we start with a product fibration $E=$ $|\mathcal{L}|_{p}^{\wedge} \times B \Gamma$, then we end up with $\left(S, \mathcal{F}^{\prime}, \mathcal{L}^{\prime}\right)=(S, \mathcal{F}, \mathcal{L})\left(\right.$ and $\left.E_{p}^{\wedge} \simeq|\mathcal{L}|_{p}^{\wedge}\right)$.

We do not know whether the index prime to $p$ analog of Corollary 4.8 holds. The key point in the proof of Corollary 4.8 is the observation that if $G$ is a finite group, whose fusion system at $p$ contains a normal subsystem of index $p^{m}$, then $G$ contains a normal subgroup of index $p^{m}$. This is not true for subsystems of index prime to $p$. For example, the fusion system at the prime 3 of the simple groups $J_{4}$ and $R u$ has a subsystem of index 2 . 


\section{Central extensions of Fusion systems And Linking Systems}

Let $\mathcal{F}$ be a fusion system over a $p$-group $S$. We say that a subgroup $A \leq S$ is central in $\mathcal{F}$ if $C_{\mathcal{F}}(A)=\mathcal{F}$, where $C_{\mathcal{F}}(A)$ is the centralizer fusion system defined in BLO2, Definition A.3]. Thus $A$ is central in $\mathcal{F}$ if $A \leq Z(S)$, and each morphism $\varphi \in \operatorname{Hom}_{\mathcal{F}}(P, Q)$ in $\mathcal{F}$ extends to a morphism $\bar{\varphi} \in \operatorname{Hom}_{\mathcal{F}}(P A, Q A)$ such that $\left.\bar{\varphi}\right|_{A}=\operatorname{Id}_{A}$.

In this section, we first study quotients of fusion systems, and of $p$-local finite groups, by central subgroups. Afterwards, we will invert this procedure, and study central extensions of fusion systems and $p$-local finite groups.

6.1. Central quotients of fusion and linking systems. We first note that every saturated fusion system $\mathcal{F}$ contains a unique maximal central subgroup, which we regard as the center of $\mathcal{F}$.

Proposition 6.1. For any saturated fusion system $\mathcal{F}$ over a p-group $S$, define

$$
Z_{\mathcal{F}}(S)=\left\{x \in Z(S) \mid \varphi(x)=x, \text { all } \varphi \in \operatorname{Mor}\left(\mathcal{F}^{c}\right)\right\}=\underset{\mathcal{F}^{c}}{\lim } Z(-):
$$

the inverse limit of the centers of all $\mathcal{F}$-centric subgroups of $S$. Then $Z_{\mathcal{F}}(S)$ is the center of $\mathcal{F}$ : it is central in $\mathcal{F}$, and contains all other central subgroups.

Proof. By definition, if $A$ is central in $\mathcal{F}$, then $A \leq Z(S)$, and any morphism in $\mathcal{F}$ between subgroups containing $A$ restricts to the identity on $A$. Since all $\mathcal{F}$-centric subgroups contain $Z(S)$, this shows that $A \leq Z_{\mathcal{F}}(S)$.

By Alperin's fusion theorem (Theorem 1.5(a)), each morphism in $\mathcal{F}$ is a composite of restrictions of morphisms between $\mathcal{F}$-centric subgroups. In particular, each morphism is a restriction of a morphism between subgroups containing $Z_{\mathcal{F}}(S)$ which is the identity on $Z_{\mathcal{F}}(S)$, and thus $Z_{\mathcal{F}}(S)$ is central in $\mathcal{F}$.

The center of a fusion system $\mathcal{F}$ has already appeared when studying mapping spaces of classifying spaces associated to $\mathcal{F}$. By [BLO2, Theorem 8.1], for any $p$-local finite group $(S, \mathcal{F}, \mathcal{L})$,

$$
\operatorname{Map}\left(|\mathcal{L}|_{p}^{\wedge},|\mathcal{L}|_{p}^{\wedge}\right)_{\mathrm{Id}} \simeq B Z_{\mathcal{F}}(S)
$$

We next define the quotient of a fusion system by a central subgroup.

Definition 6.2. Let $\mathcal{F}$ be a fusion system over a $p$-group $S$, and let $A$ be a central subgroup. Define $\mathcal{F} / A$ to be the fusion system over $S / A$ with morphism sets

$$
\operatorname{Hom}_{\mathcal{F} / A}(P / A, Q / A)=\operatorname{Im}\left[\operatorname{Hom}_{\mathcal{F}}(P, Q) \longrightarrow \operatorname{Hom}(P / A, Q / A)\right] .
$$

By [BLO2, Lemma 5.6], if $\mathcal{F}$ is a saturated fusion system over a $p$-group $S$, and $A \leq Z_{\mathcal{F}}(S)$, then $\mathcal{F} / A$ is also saturated as a fusion system over $S / A$. We now want to study the opposite question: if $\mathcal{F} / A$ is saturated, is $\mathcal{F}$ also saturated? The following example shows that it is very easy to construct counterexamples to this. In fact, under a mild hypothesis on $S$, if a fusion system over $S / A$ has the form $\mathcal{F} / A$ for any fusion system $\mathcal{F}$ over $S$, then it has that form for a fusion system $\mathcal{F}$ which is not saturated.

Example 6.3. Fix a $p$-group $S$ and a central subgroup $A \leq Z(S)$. Assume there is $\alpha \in \operatorname{Aut}(S) \backslash \operatorname{Inn}(S)$ such that $\left.\alpha\right|_{A}=\operatorname{Id}_{A}$ and $\alpha$ induces the identity on $S / A$. Let $\overline{\mathcal{F}}$ be a saturated fusion system over $S / A$, and assume there is some fusion system 
$\mathcal{F}_{0}$ over $S$ such that $\mathcal{F}_{0} / A=\overline{\mathcal{F}}$. Let $\mathcal{F} \supseteq \mathcal{F}_{0}$ be the fusion system over $S$ defined by setting

$$
\begin{aligned}
\operatorname{Hom}_{\mathcal{F}}(P, Q)=\{\varphi \in \operatorname{Hom}(P, Q) \mid \exists \bar{\varphi} \in \operatorname{Hom}(P A, Q A), \\
\left.\qquad\left.\bar{\varphi}\right|_{P}=\varphi,\left.\varphi\right|_{A}=\operatorname{Id}_{A}, \bar{\varphi} / A \in \operatorname{Hom}_{\overline{\mathcal{F}}}(P A / A, Q A / A)\right\} .
\end{aligned}
$$

Then $A$ is a central subgroup of $\mathcal{F}$, and $\mathcal{F} / A=\overline{\mathcal{F}}$. But $\mathcal{F}$ is not saturated, since $\operatorname{Aut}_{\mathcal{F}}(S)$ contains as normal subgroup the group of automorphisms of $S$ which are the identity on $A$ and on $S / A$ (a $p$-group by Lemma 1.15), and this subgroup is by assumption not contained in $\operatorname{Inn}(S)$. Hence $\operatorname{Aut}_{S}(S)$ is not a Sylow subgroup of $\operatorname{Aut}_{\mathcal{F}}(S)$.

The hypotheses of the above example are satisfied, for example, by any pair of $p$-groups $1 \neq A \supsetneqq S$ with $A \leq Z(S)$, such that $S$ is abelian, or more generally such that $A \cap[S, S]=1$.

We will now describe conditions which allow us to say when $\mathcal{F}$ is saturated. Before doing so, in the next two lemmas, we first compare properties of subgroups in $\mathcal{F}$ with those of subgroups of $\mathcal{F} / A$, when $\mathcal{F}$ is a fusion system with central subgroup $A$. This is done under varying assumptions as to whether $\mathcal{F}$ or $\mathcal{F} / A$ is saturated.

Lemma 6.4. The following hold for any fusion system $\mathcal{F}$ over a p-group $S$, and any subgroup $A \leq Z(S)$ central in $\mathcal{F}$.

(a) If $A \leq P \leq S$ and $P / A$ is $\mathcal{F} / A$-centric, then $P$ is $\mathcal{F}$-centric.

(b) If $\mathcal{F} / A$ is saturated, and $P \leq S$ is $\mathcal{F}$-quasicentric, then $P A / A$ is $\mathcal{F} / A$ quasicentric.

(c) If $\mathcal{F}$ and $\mathcal{F} / A$ are both saturated and $P / A \leq S / A$ is $\mathcal{F} / A$-quasicentric, then $P$ is $\mathcal{F}$-quasicentric.

Proof. For each $P \leq S$ containing $A$, let $\widetilde{C}_{S}(P) \leq S$ be the subgroup such that $\widetilde{C}_{S}(P) / A=C_{S / A}(P / A)$. Let

$$
\eta_{P}: \widetilde{C}_{S}(P) \longrightarrow \operatorname{Hom}(P, A)
$$

be the homomorphism $\eta_{P}(x)(g)=[x, g]$ for $x \in \widetilde{C}_{S}(P)$ and $g \in P$. Thus $\operatorname{Ker}\left(\eta_{P}\right)=$ $C_{S}(P)$.

For each $P \leq S$ containing $A$, set

$$
\Gamma_{P}=\operatorname{Ker}\left[\operatorname{Aut}_{\mathcal{F}}(P) \longrightarrow \operatorname{Aut}_{\mathcal{F} / A}(P / A)\right] .
$$

Since every $\alpha \in \operatorname{Aut}_{\mathcal{F}}(P)$ is the identity on $A, \Gamma_{P}$ is a $p$-group by Lemma 1.15,

(a) If $g, g^{\prime}$ commute in $S$, then their images commute in $S / A$. Thus for all $Q \leq S, C_{S}(Q) / A \leq C_{S / A}(Q / A)$.

Assume $P / A$ is $\mathcal{F} / A$-centric. Then for each $P^{\prime}$ which is $\mathcal{F}$-conjugate to $P, P^{\prime} / A$ is $\mathcal{F} / A$-conjugate to $P / A$, and hence $C_{S}\left(P^{\prime}\right) / A \leq C_{S / A}\left(P^{\prime} / A\right) \leq P^{\prime} / A$. Thus $P$ is $\mathcal{F}$-centric.

(b) Fix $P \leq S$ such that $P$ is $\mathcal{F}$-quasicentric. Since $C_{S}\left(P^{\prime} A\right)=C_{S}\left(P^{\prime}\right)$ for all $P^{\prime}$ which is $\mathcal{F}$-conjugate to $P, P^{\prime} A$ is fully centralized in $\mathcal{F}$ if and only if $P^{\prime}$ is. Also, $C_{\mathcal{F}}\left(P^{\prime}\right)=C_{\mathcal{F}}\left(P^{\prime} A\right)$ for such $P^{\prime}$, and this shows that $P A$ is also $\mathcal{F}$-quasicentric. So after replacing $P$ by $P A$, if necessary, we can assume $P \geq A$.

Choose $P^{\prime} / A$ which is fully centralized in $\mathcal{F} / A$ and $\mathcal{F} / A$-conjugate to $P / A$. Then $P^{\prime}$ is $\mathcal{F}$-conjugate to $P$, hence still $\mathcal{F}$-quasicentric. So upon replacing $P$ by 
$P^{\prime}$, we are reduced to showing that $P / A$ is $\mathcal{F} / A$-quasicentric when $P \geq A, P$ is $\mathcal{F}$-quasicentric, and $P / A$ is fully centralized.

For any $P^{\prime}$ which is $\mathcal{F}$-conjugate to $P$, there is a morphism

$$
\varphi / A \in \operatorname{Hom}_{\mathcal{F} / A}\left(\left(P^{\prime} \cdot \widetilde{C}_{S}\left(P^{\prime}\right)\right) / A,\left(P \cdot \widetilde{C}_{S}(P)\right) / A\right)
$$

which sends $P^{\prime} / A$ to $P / A$ (by axiom (II) for the saturated fusion system $\mathcal{F} / A$ ). Then $\varphi$ restricts to a morphism from $C_{S}\left(P^{\prime}\right)$ to $C_{S}(P)$. Thus $\left|C_{S}\left(P^{\prime}\right)\right| \leq\left|C_{S}(P)\right|$ for all $P^{\prime}$ which is $\mathcal{F}$-conjugate to $P$, and this proves that $P$ is fully centralized in $\mathcal{F}$.

If $P / A$ is not $\mathcal{F} / A$-quasicentric, then by Lemma 1.6 (and since $\mathcal{F} / A$ is saturated), there is some $Q / A \leq P / A \cdot C_{S / A}(P / A)$ containing $P / A$, and some $\alpha \in \operatorname{Aut}_{\mathcal{F}}(Q)$, such that $\operatorname{Id} \neq \alpha / A \in \operatorname{Aut}_{\mathcal{F} / A}(Q / A)$ has order prime to $p$ and $\alpha / A$ is the identity on $P / A$. Since $\alpha$ is also the identity on $A$, Lemma 1.15 implies that $\left.\alpha\right|_{P}=\operatorname{Id}_{P}$. Set $Q^{\prime}=Q \cap \widetilde{C}_{S}(P)$. Then $\alpha\left(Q^{\prime}\right)=Q^{\prime}$, and $\eta_{P} \circ \alpha=\eta_{P}$ since $\alpha$ is the identity on $P \geq A$. Thus $\alpha$ induces the identity on $Q^{\prime} / C_{Q^{\prime}}(P)$ since $\operatorname{Ker}\left(\eta_{P}\right)=C_{S}(P)$. Since $\alpha$ has order prime to $p$, Lemma 1.15 now implies that $\left.\alpha\right|_{C_{Q^{\prime}}(P)} \neq$ Id. Thus $C_{Q^{\prime}}(P) \leq C_{S}(P)$ and $\left.\alpha\right|_{P \cdot C_{Q^{\prime}}(P)}$ is a nontrivial automorphism in $C_{\mathcal{F}}(P)$ of order prime to $p$. Since $P$ is fully centralized in $\mathcal{F}$, this implies (by definition) that $C_{\mathcal{F}}(P)$ is not the fusion system of $C_{S}(P)$, and hence that $P$ is not $\mathcal{F}$-quasicentric.

(c) Now assume that $\mathcal{F}$ and $\mathcal{F} / A$ are both saturated, and that $P / A \leq S / A$ is $\mathcal{F} / A$-quasicentric. If $P$ is not $\mathcal{F}$-quasicentric, and $P^{\prime}$ is $\mathcal{F}$-conjugate to $P$ and fully centralized in $\mathcal{F}$, then by Lemma 1.6(b), there is some $P^{\prime} \leq Q \leq P^{\prime} \cdot C_{S}\left(P^{\prime}\right)$ and some $\operatorname{Id} \neq \alpha \in \operatorname{Aut}_{\mathcal{F}}(Q)$ such that $\left.\alpha\right|_{P^{\prime}}=\operatorname{Id}_{P^{\prime}}$ and $\alpha$ has order prime to $p$. Then $Q / A \leq\left(P^{\prime} / A\right) \cdot C_{S / A}\left(P^{\prime} / A\right), \alpha / A \in \operatorname{Aut}_{\mathcal{F} / A}(Q / A)$ also has order prime to $p$, and so $\alpha / A \neq$ Id by Lemma 1.15] again. But by Lemma [1.6(a), this contradicts the assumption that $P / A$ is $\mathcal{F} / A$-quasicentric.

In the next lemma, we compare conditions for being fully normalized in $\mathcal{F}$ and in $\mathcal{F} / A$.

Lemma 6.5. The following hold for any fusion system $\mathcal{F}$ over a p-group $S$, and any subgroup $A \leq Z(S)$ central in $\mathcal{F}$.

(a) Assume $\mathcal{F}$ is saturated. Then for all $P, Q \leq S$ containing $A$ such that $P$ is fully normalized in $\mathcal{F}$, if $\varphi, \varphi^{\prime} \in \operatorname{Hom}_{\mathcal{F}}(P, Q)$ are such that $\varphi / A=\varphi^{\prime} / A$, then $\varphi^{\prime}=\varphi \circ c_{x}$ for some $x \in N_{S}(P)$ such that $x A \in C_{S / A}(P / A)$.

(b) Assume $\mathcal{F} / A$ is saturated, and let $P, P^{\prime} \leq S$ be $\mathcal{F}$-conjugate subgroups which contain $A$. Then $P$ is fully normalized in $\mathcal{F}$ if and only if $P / A$ is fully normalized in $\mathcal{F} / A$. Moreover, if $P$ is fully normalized in $\mathcal{F}$, then there is $\psi \in \operatorname{Hom}_{\mathcal{F}}\left(N_{S}\left(P^{\prime}\right), N_{S}(P)\right)$ such that $\psi\left(P^{\prime}\right)=P$.

Proof. (a) Assume $\mathcal{F}$ is saturated, and fix $P, Q \leq S$ containing $A$ such that $P$ is fully normalized in $\mathcal{F}$. Let $\varphi, \varphi^{\prime} \in \operatorname{Hom}_{\mathcal{F}}(P, Q)$ be such that $\varphi / A=\varphi^{\prime} / A$. Then $\operatorname{Im}(\varphi)=\operatorname{Im}\left(\varphi^{\prime}\right)$. Set $\alpha=\varphi^{-1} \circ \varphi^{\prime} \in \operatorname{Aut}_{\mathcal{F}}(P) ;$ then $\varphi^{\prime}=\varphi \circ \alpha$, and $\alpha / A=\operatorname{Id}_{P / A}$. Thus

$$
\alpha \in \operatorname{Ker}\left[\operatorname{Aut}_{\mathcal{F}}(P) \longrightarrow \operatorname{Aut}_{\mathcal{F} / A}(P / A)\right],
$$

which is a normal $p$-subgroup by Lemma 1.15. Also, $\operatorname{Aut}_{S}(P) \in \operatorname{Syl}_{p}\left(\operatorname{Aut}_{\mathcal{F}}(P)\right)$ since $P$ is fully normalized, and so $\alpha \in \operatorname{Aut}_{S}(P)$. Thus $\alpha \in c_{x}$ for some $x \in N_{S}(P)$, and $x A \in C_{S / A}(P / A)$ since $\alpha / A=\operatorname{Id}_{P / A}$. 
(b) We are now assuming that $\mathcal{F} / A$ is saturated. Assume first that $P / A$ is fully normalized in $\mathcal{F} / A$. Then by Lemma 1.3, there is a morphism

$$
\varphi_{0} \in \operatorname{Hom}_{\mathcal{F} / A}\left(N_{S / A}\left(P^{\prime} / A\right), N_{S / A}(P / A)\right)
$$

such that $\varphi_{0}\left(P^{\prime} / A\right)=P / A$, and this lifts to $\varphi \in \operatorname{Hom}_{\mathcal{F}}\left(N_{S}\left(P^{\prime}\right), N_{S}(P)\right)$ such that $\varphi\left(P^{\prime}\right)=P$. Therefore $\left|N_{S}\left(P^{\prime}\right)\right| \leq\left|N_{S}(P)\right|$ for any $P^{\prime}$ which is $\mathcal{F}$-conjugate to $P$ and $P$ is fully normalized in $\mathcal{F}$. This also proves that the last statement in (b) holds, once we know that $P / A$ is fully normalized.

Assume now that $P$ is fully normalized in $\mathcal{F}$; we want to show that $P / A$ is fully normalized in $\mathcal{F} / A$. Fix $P^{\prime}$ which is $\mathcal{F}$-conjugate to $P$ and such that $P^{\prime} / A$ is fully normalized in $\mathcal{F} / A$. By Lemma 1.3 again, there exists

$$
\varphi_{0} \in \operatorname{Hom}_{\mathcal{F} / A}\left(N_{S / A}(P / A), N_{S / A}\left(P^{\prime} / A\right)\right)
$$

such that $\varphi_{0}(P / A)=P^{\prime} / A$. Then $\varphi_{0}=\varphi / A$ for some $\varphi \in \operatorname{Hom}_{\mathcal{F}}\left(N_{S}(P), N_{S}\left(P^{\prime}\right)\right)$, and $\varphi$ is an isomorphism since $P$ is fully normalized in $\mathcal{F}$. Thus $\varphi_{0}$ is an isomorphism, and hence $P / A$ is fully normalized in $\mathcal{F} / A$.

Thus if $P$ is fully normalized in $\mathcal{F}$, then $P / A$ is also fully normalized, and we have already seen that the last statement in (b) holds in this case.

We are now ready to give conditions under which we show that $\mathcal{F}$ is saturated if $\mathcal{F} / A$ is saturated. As in [5A1, §2], for any fusion system $\mathcal{F}$ over a $p$-group $S$, and any set $\mathcal{H}$ of subgroups of $S$, we say that $\mathcal{F}$ is $\mathcal{H}$-generated if each morphism in $\mathcal{F}$ is a composite of restrictions of morphisms between subgroups in $\mathcal{H}$.

Proposition 6.6. Let $A$ be a central subgroup of a fusion system $\mathcal{F}$ over a p-group $S$, such that $\mathcal{F} / A$ is a saturated fusion system. Let $\mathcal{H}$ be any set of subgroups of $S$, closed under $\mathcal{F}$-conjugacy and overgroups, which contains all $\mathcal{F}$-centric subgroups of $S$. Assume

(a) $\operatorname{Ker}\left[\operatorname{Aut}_{\mathcal{F}}(P) \longrightarrow \operatorname{Aut}_{\mathcal{F} / A}(P / A)\right] \leq \operatorname{Aut}_{S}(P)$ for each $P \in \mathcal{H}$ which is fully normalized in $\mathcal{F}$; and

(b) $\mathcal{F}$ is $\mathcal{H}$-generated.

Then $\mathcal{F}$ is saturated.

Proof. Let $\mathcal{H}_{0}$ be the set of all $P \in \mathcal{H}$ such that $P \geq A$. Since $A$ is central, each morphism $\varphi \in \operatorname{Hom}_{\mathcal{F}}(P, Q)$ extends to some $\bar{\varphi} \in \operatorname{Hom}_{\mathcal{F}}(P A, Q A)$ such that $\left.\bar{\varphi}\right|_{A}=\operatorname{Id}_{A}$. Thus $\mathcal{F}$ is $\mathcal{H}_{0}$-generated if it is $\mathcal{H}$-generated. So upon replacing $\mathcal{H}$ by $\mathcal{H}_{0}$, we can assume all subgroups in $\mathcal{H}$ contain $A$.

By assumption, $\mathcal{H}$ contains all $\mathcal{F}$-centric subgroups of $S$. So by Theorem 1.5(b), it suffices to check that axioms (I) and (II) hold for all $P \in \mathcal{H}$. For all $P \leq S$ containing $A$, we write

$$
\Gamma_{P} \stackrel{\text { def }}{=} \operatorname{Ker}\left[\operatorname{Aut}_{\mathcal{F}}(P) \longrightarrow \operatorname{Aut}_{\mathcal{F} / A}(P / A)\right] .
$$

(I) Assume $P \in \mathcal{H}$ is fully normalized in $\mathcal{F}$. Then $P / A$ is fully normalized in $\mathcal{F} / A$ by Lemma 6.5(b). By assumption, $\Gamma_{P} \leq \operatorname{Aut}_{S}(P)$. Hence

$$
\left[\operatorname{Aut}_{\mathcal{F}}(P): \operatorname{Aut}_{S}(P)\right]=\left[\operatorname{Aut}_{\mathcal{F} / A}(P / A): \operatorname{Aut}_{S / A}(P / A)\right] \text {, }
$$

and $\operatorname{Aut}_{S}(P) \in \operatorname{Syl}_{p}\left(\operatorname{Aut}_{\mathcal{F}}(P)\right)$ since $\operatorname{Aut}_{S / A}(P / A) \in \operatorname{Syl}_{p}\left(\operatorname{Aut}_{\mathcal{F} / A}(P / A)\right)$.

Assume $P^{\prime} \leq S$ is $\mathcal{F}$-conjugate to $P$ and fully centralized in $\mathcal{F}$. By Lemma 6.5(b) again, there is $\psi \in \operatorname{Hom}_{\mathcal{F}}\left(N_{S}\left(P^{\prime}\right), N_{S}(P)\right)$ such that $\psi\left(P^{\prime}\right)=P$. Then $\psi\left(C_{S}\left(P^{\prime}\right)\right) \leq C_{S}(P)$, so $P$ is also fully centralized. 
(II) Assume $\varphi \in \operatorname{Hom}_{\mathcal{F}}(P, S)$ is such that $P \in \mathcal{H}$ and $\varphi(P)$ is fully centralized. Set

$$
N_{\varphi}=\left\{g \in N_{S}(P) \mid \varphi c_{g} \varphi^{-1} \in \operatorname{Aut}_{S}(\varphi(P))\right\}
$$

as usual. Then $N_{\varphi} / A \leq N_{\varphi / A}$.

Assume first that $\varphi(P)$ is fully normalized in $\mathcal{F}$. Then $\varphi(P) / A$ is fully normalized in $\mathcal{F} / A$ by Lemma 6.5(b), and hence also fully centralized. So by (II), applied to the saturated fusion system $\mathcal{F} / A$, there is $\widehat{\varphi} \in \operatorname{Hom}_{\mathcal{F}}\left(N_{\varphi / A}, S / A\right)$ such that $\left.\widehat{\varphi}\right|_{(P / A)}=\varphi / A$. Let $\bar{\varphi} \in \operatorname{Hom}_{\mathcal{F}}\left(N_{\varphi}, S\right)$ be a lift of $\widehat{\varphi}$; then $\left(\left.\bar{\varphi}\right|_{P}\right) / A=\varphi / A$ and $\bar{\varphi}(P)=\varphi(P)$. So there is $\alpha=\varphi \circ\left(\left.\bar{\varphi}\right|_{P}\right)^{-1} \in \Gamma_{\varphi(P)} \leq \operatorname{Aut}_{\mathcal{F}}(\varphi(P))$ such that $\left.\alpha \circ \bar{\varphi}\right|_{P}=\varphi$. By (a), there is $x \in N_{S}(\varphi(P))$ such that $\alpha=c_{x}$; then $c_{x} \circ \bar{\varphi}$ lies in $\operatorname{Hom}_{\mathcal{F}}\left(N_{\varphi}, S\right)$ and extends $\varphi$.

It remains to prove the general case. Choose $P^{\prime}$ which is fully normalized in $\mathcal{F}$ and $\mathcal{F}$-conjugate to $P$. By Lemma 6.5 (b), there is $\psi \in \operatorname{Hom}_{\mathcal{F}}\left(N_{S}(\varphi(P)), N_{S}\left(P^{\prime}\right)\right)$ such that $\psi(\varphi(P))=P^{\prime}$. Then $N_{\varphi} \leq N_{\psi \varphi}$. Since $\psi \varphi(P)=P^{\prime}$ is fully normalized, $\psi \varphi$ extends to some $\bar{\psi} \in \operatorname{Hom}_{\mathcal{F}}\left(N_{\varphi}, N_{S}\left(P^{\prime}\right)\right)$. We will show that $\operatorname{Im}(\bar{\psi}) \leq \operatorname{Im}(\psi)$, and thus there is $\bar{\varphi} \in \operatorname{Hom}_{\mathcal{F}}\left(N_{\varphi}, N_{S}(\varphi(P))\right)$ such that $\left.\bar{\varphi}\right|_{P}=\varphi$.

For each $g \in N_{\varphi}$, choose $x \in N_{S}(\varphi(P))$ such that $\varphi c_{g} \varphi^{-1}=c_{x}$; then we obtain $c_{\psi(x)}=\psi c_{x} \psi^{-1}=c_{\bar{\psi}(g)}$, and so $\psi(x) \bar{\psi}(g)^{-1} \in C_{S}\left(P^{\prime}\right)$. Since $\varphi(P)$ is fully centralized in $\mathcal{F}, \psi\left(C_{S}(\varphi(P))\right)=C_{S}\left(P^{\prime}\right)$, and thus $\bar{\psi}(g) \in \operatorname{Im}(\psi)$.

For example, one can take as the set $\mathcal{H}$ in Proposition 6.6 either the set of subgroups $P \leq S$ containing $A$ such that $P / A$ is $\mathcal{F} / A$-quasicentric (by Lemma 6.4(b)), or the set of $\mathcal{F}$-centric subgroups of $S$.

Now let $(S, \mathcal{F}, \mathcal{L})$ be a $p$-local finite group. One can also define a centralizer linking system $C_{\mathcal{L}}(A)$ when $A \leq S$ is fully centralized [BLO2, Definition 2.4]. Since this is always a linking system associated to $C_{\mathcal{F}}(A), A$ is central in $\mathcal{L}$ (i.e., $C_{\mathcal{L}}(A)=\mathcal{L}$ ) if and only if $A$ is central in $\mathcal{F}$. So from now on, by a central subgroup of $(S, \mathcal{F}, \mathcal{L})$, we just mean a subgroup $A \leq Z(S)$ which is central in $\mathcal{F}$.

Definition 6.7. Let $(S, \mathcal{F}, \mathcal{L})$ be a $p$-local finite group with a central subgroup $A$. Define $\mathcal{L} / A$ to be the category with objects the subgroups $P / A$ for $P \in \operatorname{Ob}(\mathcal{L})$ (i.e., such that $P$ is $\mathcal{F}$-centric), and with morphism sets

$$
\operatorname{Mor}_{\mathcal{L} / A}(P / A, Q / A)=\operatorname{Mor}_{\mathcal{L}}(P, Q) / \delta_{P}(A) .
$$

Let $(\mathcal{L} / A)^{c} \subseteq \mathcal{L} / A$ be the full subcategory with object set the $\mathcal{F} / A$-centric subgroups in $S / A$.

Similarly, if $\mathcal{L}^{q}$ is the associated quasicentric linking system, then define $\mathcal{L}^{q} / A$ to be the category with objects the $\mathcal{F} / A$-quasicentric subgroups of $S / A$ - equivalently, the subgroups $P A / A$ for $P \in \operatorname{Ob}\left(\mathcal{L}^{q}\right)$ - and with morphisms

$$
\operatorname{Mor}_{\mathcal{L}^{q} / A}(P / A, Q / A)=\operatorname{Mor}_{\mathcal{L}^{q}}(P, Q) / \delta_{P}(A) .
$$

Note that by Lemma 6.4(a), (c), for any $P / A \leq S / A$, if $P / A$ is $\mathcal{F} / A$-centric or $\mathcal{F} / A$-quasicentric, then $P$ is $\mathcal{F}$-centric or $\mathcal{F}$-quasicentric, respectively. Thus the categories $(\mathcal{L} / A)^{c} \subseteq \mathcal{L} / A$ and $\mathcal{L}^{q} / A$ are well defined.

We are now ready to prove our main theorem about quotient fusion and linking systems.

Theorem 6.8. Let $A$ be a central subgroup of a p-local finite group $(S, \mathcal{F}, \mathcal{L})$ with associated quasicentric linking system $\mathcal{L}^{q}$. Let $\mathcal{L}_{\geq A}^{q} \subseteq \mathcal{L}^{q}$ be the full subcategory with 
objects those $\mathcal{F}$-quasicentric subgroups of $S$ which contain $A$. Then the following hold:

(a) $\left(S / A, \mathcal{F} / A,(\mathcal{L} / A)^{c}\right)$ is again a $p$-local finite group, and $\mathcal{L}^{q} / A$ is a quasicentric linking system associated to $\mathcal{F} / A$.

(b) The inclusions of categories induce homotopy equivalences $|\mathcal{L}| \simeq\left|\mathcal{L}_{\geq A}^{q}\right| \simeq$ $\left|\mathcal{L}^{q}\right|$ and $\left|(\mathcal{L} / A)^{c}\right| \simeq|\mathcal{L} / A| \simeq\left|\mathcal{L}^{q} / A\right|$.

(c) The functor $\tau: \mathcal{L}^{q} \rightarrow \mathcal{L}^{q} / A$, defined by $\tau(P)=P A / A$ and with the obvious maps on morphisms, induces principal fibration sequences

$$
B A \longrightarrow|\mathcal{L}| \stackrel{|\tau|}{\longrightarrow}|\mathcal{L} / A| \quad \text { and } \quad B A \longrightarrow\left|\mathcal{L}_{\geq A}^{q}\right| \stackrel{|\tau|}{\longrightarrow}\left|\mathcal{L}^{q} / A\right|
$$

which remain principal fibration sequences after p-completion.

Proof. (a) The first statement is shown in [BLO2, Lemma 5.6] when $|A|=p$, and the general case follows by iteration. So we need only prove that $\mathcal{L}^{q} / A$ is a quasicentric linking system associated to $\mathcal{F} / A$. Axioms $(\mathrm{B})_{q},(\mathrm{C})_{q}$, and $(\mathrm{D})_{q}$ for $\mathcal{L}^{q} / A$ follow immediately from those axioms applied to $\mathcal{L}^{q}$, so it remains only to prove $(\mathrm{A})_{q}$.

Let $\mathcal{H}$ be the set of subgroups $P \leq S$ such that $A \leq P$ and $P / A$ is $\mathcal{F} / A$ quasicentric and fix $P, Q \in \mathcal{H}$. By construction, $C_{S / A}(P / A)$ acts freely on the morphism set $\operatorname{Mor}_{\mathcal{L}^{q} / A}(P / A, Q / A)$, and induces a surjection

$$
\operatorname{Mor}_{\mathcal{L}^{q} / A}(P / A, Q / A) / C_{S / A}(P / A) \longrightarrow \operatorname{Hom}_{\mathcal{F} / A}(P / A, Q / A) \text {. }
$$

We must show that this is a bijection whenever $P / A$ is fully centralized in $\mathcal{F} / A$. Since any other fully centralized subgroup in the same $\mathcal{F}$-conjugacy class has a centralizer of the same order, it suffices to show this when $P / A$ is fully normalized in $\mathcal{F} / A$; or equivalently (by Lemma 6.5 (b)) when $P$ is fully normalized in $\mathcal{F}$.

Let $\widetilde{C}_{S}(P) \leq S$ be the subgroup such that $\widetilde{C}_{S}(P) / A=C_{S / A}(P / A)$. Fix any $\mathcal{F} / A$-quasicentric subgroup $Q / A$, and consider the following sequence of maps:

$$
\operatorname{Mor}_{\mathcal{L}^{q}}(P, Q) \longrightarrow \operatorname{Hom}_{\mathcal{F}}(P, Q) \longrightarrow \operatorname{Hom}_{\mathcal{F} / A}(P / A, Q / A) .
$$

Since $\mathcal{L}^{q}$ is the quasicentric linking system of $\mathcal{F}$, by property $(\mathrm{A})_{q}$ the first map is the orbit map of the action of $C_{S}(P)$. The second is the orbit map for the action of $\operatorname{Aut}_{\widetilde{C}_{S}(P)}(P)$ by Lemma 6.5(a). Thus the composite is the orbit map for the free action of $\widetilde{C}_{S}(P)$. It now follows that $\widetilde{C}_{S}(P) / A \cong C_{S / A}(P / A)$ acts freely on $\operatorname{Mor}_{\mathcal{L}^{q} / A}(P / A, Q / A)=\operatorname{Mor}_{\mathcal{L}^{q}}(P, Q) / A$ with orbit set $\operatorname{Hom}_{\mathcal{F} / A}(P / A, Q / A)$.

(b) These homotopy equivalences are all special cases of Proposition 1.12(a).

(c) For any category $\mathcal{C}$ and any $n \geq 0$, let $\mathcal{C}_{n}$ denote the set of $n$-simplices in the nerve of $\mathcal{C}$; i.e., the set of composable $n$-tuples of morphisms. For each $n \geq 0$, the group $\mathcal{B}(A)_{n}$ acts freely on $\left(\mathcal{L}_{\geq A}^{q}\right)_{n}$ : an element $\left(a_{1}, \ldots, a_{n}\right)$ acts by composing the $i$-th component with $\delta_{P}\left(a_{i}\right)$ for appropriate $P$. This action commutes with the face and degeneracy maps, and its orbit set is $\left(\mathcal{L}^{q} / A\right)_{n}$. It follows that the projection of $\left|\mathcal{L}_{\geq A}^{q}\right|$ onto $\left|\mathcal{L}^{q} / A\right|$ (and of $|\mathcal{L}|$ onto $|\mathcal{L} / A|$ ) is a principal fibration with fiber the topological group $B A=|\mathcal{B}(A)|$ (see, e.g., [May, §§18-20] or [GJ, Corollary V.2.7]).

By the principal fibration lemma of Bousfield and Kan [BK, II.2.2], these sequences are still principal fibration sequencesafter $p$-completion. 
6.2. Central extensions of $p$-local finite groups. We first make it more precise what we mean by this.

Definition 6.9. A central extension of a (saturated) fusion system $\mathcal{F}$ over a $p$ group $S$ by an abelian $p$-group $A$ consists of a (saturated) fusion system $\widetilde{\mathcal{F}}$ over a $p$-group $\widetilde{S}$, together with an inclusion $A \leq Z(\widetilde{S})$, such that $A$ is a central subgroup of $\widetilde{\mathcal{F}}, \widetilde{S} / A \cong S$, and $\widetilde{\mathcal{F}} / A \cong \mathcal{F}$ as fusion systems over $S$.

Similarly, a central extension of a $p$-local finite group $(S, \mathcal{F}, \mathcal{L})$ by an abelian group $A$ consists of a $p$-local finite group $(\widetilde{S}, \widetilde{\mathcal{F}}, \widetilde{\mathcal{L}})$, together with an inclusion $A \leq Z_{\widetilde{\mathcal{F}}}(\widetilde{S})$, such that $\left(\widetilde{S} / A, \widetilde{\mathcal{F}} / A,(\widetilde{\mathcal{L}} / A)^{c}\right) \cong(S, \mathcal{F}, \mathcal{L})$.

Extensions of categories were defined and studied by Georges Hoff in [Hf], where he proves that they are classified by certain Ext-groups. We will deal with one particular case of this. Hoff's theorem implies that an extension of categories of the type $\mathcal{B}(A) \longrightarrow \widetilde{\mathcal{L}}_{\geq A}^{q} \stackrel{\tau}{\longrightarrow} \mathcal{L}^{q}$ is classified by an element in ${\overleftarrow{\mathcal{L}^{q}}}_{\lim ^{2}}(A)$. What this extension really means is that $\mathcal{L}^{q}$ is a quotient category of $\widetilde{\mathcal{L}}_{\geq A}^{q}$, where each morphism set in $\widetilde{\mathcal{L}}_{\geq A}^{q}$ admits a free action of $A$ with orbit set the corresponding morphism set in $\mathcal{L}^{q}$. Also, $\lim ^{2}(A)$ means the second derived functor of the limit of the constant functor which sends each object of $\mathcal{L}^{q}$ to $A$ and each morphism to $\operatorname{Id}_{A}$.

We regard $A$ as an additive group. Fix an element $[\omega] \in{\overleftarrow{\mathcal{L}^{q}}}_{\lim ^{2}}^{2}(A)$, where $\omega$ is a (reduced) 2-cocyle. Thus $\omega$ is a function from pairs of composable morphisms in $\mathcal{L}^{q}$ to $A$ such that $\omega(f, g)=0$ if $f$ or $g$ is an identity morphism, and such that for any triple $f, g, h$ of composable morphisms, the cocycle condition is satisfied:

$$
d \omega(f, g, h) \stackrel{\text { def }}{=} \omega(g, h)-\omega(g f, h)+\omega(f, h g)-\omega(f, g)=0 .
$$

Consider the composite $i \circ \delta_{S}: \mathcal{B S} \rightarrow \mathcal{L}^{q}$, where $\delta_{S}$ is induced by the distinguished morphism $S \rightarrow \operatorname{Aut}_{\mathcal{L}^{q}}(S)$ and $i$ is induced by the inclusion of $\operatorname{Aut}_{\mathcal{L}^{q}}(S)$ in $\mathcal{L}^{q}$ as the full subcategory with one object $S$. Then $\omega_{S}=\left(i \circ \delta_{S}\right)^{*}(\omega)$ is a 2-cocycle defined on $S$ which classifies a central extension of $p$-groups

$$
1 \longrightarrow A \longrightarrow \widetilde{S} \stackrel{\tau}{\longrightarrow} S \longrightarrow 1 \text {. }
$$

Thus $\widetilde{S}=S \times A$, with group multiplication defined by

$$
(h, b) \cdot(g, a)=\left(h g, b+a+\omega_{S}(g, h)\right),
$$

and $\tau(g, a)=g$. For each $\mathcal{F}$-quasicentric subgroup $P \leq S$, set $\widetilde{P}=\tau^{-1}(P) \leq \widetilde{S}$.

Using this cocycle $\omega$, we can define a new category $\widetilde{\mathcal{L}}_{0}$ as follows. There is one object $\widetilde{P}=\tau^{-1}(P)$ of $\widetilde{\mathcal{L}}_{0}$ for each object $P$ of $\mathcal{L}^{q}$. Morphism sets in $\widetilde{\mathcal{L}}_{0}$ are defined by setting

$$
\operatorname{Mor}_{\widetilde{\mathcal{L}}_{0}}(\widetilde{P}, \widetilde{Q})=\operatorname{Mor}_{\mathcal{L}^{q}}(P, Q) \times A .
$$

Composition in $\widetilde{\mathcal{L}}_{0}$ is defined by

$$
(g, b) \circ(f, a)=(g f, a+b+\omega(f, g)) .
$$

The associativity of this composition law follows since $d \omega=0$. Furthermore, if we choose another representative $\omega+d \mu$ where $\mu$ is a 1-cochain, the categories obtained are isomorphic. This construction comes together with a projection functor $\tau: \widetilde{\mathcal{L}}_{0} \rightarrow \mathcal{L}^{q}$. 
Finally, we define

$$
\delta_{\widetilde{S}}: S \longrightarrow \operatorname{Aut}_{\widetilde{\mathcal{L}}_{0}}(S)
$$

by setting $\delta_{\widetilde{S}}(g, a)=\left(\delta_{S}(g), a\right)$. This is a group homomorphism by definition of $\omega_{S}$.

Proposition 6.10. Let $(S, \mathcal{F}, \mathcal{L})$ be a p-local finite group, and let $\mathcal{L}^{q}$ be the associated quasicentric linking system. Fix an abelian group $A$ and a reduced 2-cocycle $\omega$ on $\mathcal{L}^{q}$ with coefficients in $A$. Let

$$
\widetilde{\mathcal{L}}_{0} \stackrel{\tau}{\longrightarrow} \mathcal{L}^{q} \quad \text { and } \quad \widetilde{S} \stackrel{\tau}{\longrightarrow} S
$$

be the induced extensions of categories and of groups, with distinguished monomorphism $\delta_{\widetilde{S}}$ as defined above.

Then there is a unique saturated fusion system $\widetilde{\mathcal{F}}$ over $\widetilde{S}$ equipped with a functor $\widetilde{\pi}: \widetilde{\mathcal{L}}_{0} \rightarrow \widetilde{\mathcal{F}}$, and also unique distinguished monomorphisms $\delta_{\widetilde{P}}: \widetilde{P} \rightarrow \operatorname{Aut}_{\widetilde{\mathcal{L}}_{0}}(P)$.

If $\widetilde{\mathcal{L}} \subseteq \widetilde{\mathcal{L}}_{0}$ denotes the full subcategory whose objects are the $\widetilde{\mathcal{F}}$-centric subgroups of $\widetilde{S}$, then $(\widetilde{S}, \widetilde{\mathcal{F}}, \widetilde{\mathcal{L}})$ is a p-local finite group with central subgroup $A \leq Z(\widetilde{S})$, such that

$$
\left(\widetilde{S} / A, \widetilde{\mathcal{F}} / A, \widetilde{\mathcal{L}}_{0} / A\right) \cong\left(S, \mathcal{F}, \mathcal{L}^{q}\right) .
$$

Also, $\widetilde{\mathcal{L}}_{0}$ extends to a quasicentric linking system $\widetilde{\mathcal{L}}^{q}$ associated to $\widetilde{\mathcal{F}}$.

Proof. Assume that inclusion morphisms $\iota_{P}$ have been chosen in the quasicentric linking system $\mathcal{L}^{q}$ associated to $(S, \mathcal{F}, \mathcal{L})$. For each $\mathcal{F}$-quasicentric $P \supsetneqq S$, define

$$
\iota_{\widetilde{P}}=\left(\iota_{P}, 0\right) \in \operatorname{Mor}_{\widetilde{\mathcal{L}}_{0}}(\widetilde{P}, \widetilde{S}) .
$$

Next, define the distinguished monomorphism

$$
\delta_{\widetilde{P}}: \widetilde{P} \cdot C_{\widetilde{S}}(\widetilde{P}) \longrightarrow \operatorname{Aut}_{\widetilde{\mathcal{L}}_{0}}(\widetilde{P})
$$

to be the unique monomorphism such that the following square commutes for each $\widetilde{P}$ and each $q \in \widetilde{P} \cdot C_{\widetilde{S}}(\widetilde{P})$ :

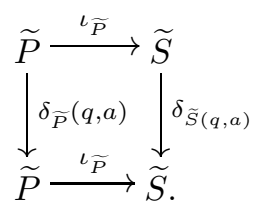

More precisely, since $\delta_{\widetilde{S}}(q, a)=\left(\delta_{S}(q), a\right)$ by definition, this means that

$$
\delta_{\widetilde{P}}(q, a)=\left(\delta_{P}(q), a+\omega\left(\iota_{P}, \delta_{S}(q)\right)-\omega\left(\delta_{P}(q), \iota_{P}\right)\right) .
$$

For each morphism $(f, a) \in \operatorname{Mor}_{\widetilde{\mathcal{L}}_{0}}(\widetilde{P}, \widetilde{Q})$, and each element $(q, b) \in \widetilde{P}$, there is a unique element $c \in A$ such that the following square commutes:

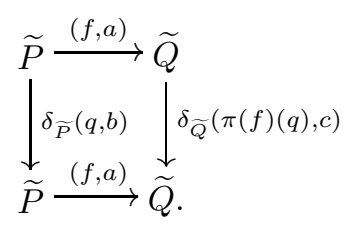

In this situation, we set

$$
\widetilde{\pi}(f, a)(q, b)=(\pi(f)(q), c) \in \widetilde{Q} .
$$


By juxtaposing squares of the form $(3)$, we see that $\widetilde{\pi}(f, a) \in \operatorname{Hom}(\widetilde{P}, \widetilde{Q})$, and that this defines a functor $\widetilde{\pi}$ from $\widetilde{\mathcal{L}}_{0}$ to the category of subgroups of $\widetilde{S}$ with monomorphisms.

Define $\widetilde{\mathcal{F}}$ to be the fusion system over $\widetilde{S}$ generated by the image of $\widetilde{\pi}$ and restrictions. By construction, the surjection $\tau: \widetilde{S} \longrightarrow S$ induces a functor $\tau_{*}: \widetilde{\mathcal{F}} \longrightarrow \mathcal{F}$ between the fusion systems, which is surjective since $\mathcal{F}$ is generated by restrictions of morphisms between $\mathcal{F}$-quasicentric subgroups (Theorem[1.5(a)). So we can identify $\mathcal{F}$ with $\widetilde{\mathcal{F}} / A$. By Lemma 6.4(b), for each $\widetilde{\mathcal{F}}$-quasicentric subgroup $P \leq \widetilde{S}, P A / A$ is $\mathcal{F}$-quasicentric. So we can extend $\widetilde{\mathcal{L}}_{0}$ to a category $\widetilde{\mathcal{L}}^{q}$ defined on all $\overline{\mathcal{F}}$-quasicentric subgroups, by setting

$$
\operatorname{Mor}_{\mathcal{L}^{q}}(P, Q)=\left\{f \in \operatorname{Mor}_{\widetilde{\mathcal{L}}_{0}}(P A, Q A) \mid \widetilde{\pi}(f)(P) \leq Q\right\} \quad \text { and } \quad \delta_{P}=\delta_{P A}
$$

for each pair $P, Q$ of $\widetilde{\mathcal{F}}$-quasicentric subgroups. We extend $\widetilde{\pi}$ to $\widetilde{\mathcal{L}}^{q}$ in the obvious way.

It remains to prove that $\widetilde{\mathcal{F}}$ is saturated, and that $\widetilde{\mathcal{L}}^{q}$ is a quasicentric linking system associated to $\widetilde{\mathcal{F}}$. In the process of doing this, we will also prove the isomorphism (1).

Let $\mathcal{H}$ be the set of subgroups $\widetilde{P}=\tau^{-1}(P) \leq \widetilde{S}$ for all $\mathcal{F}$-quasicentric subgroups $P \leq S$.

$\widetilde{\mathcal{F}}$ is saturated: We want to apply Proposition 6.6 to prove that $\widetilde{\mathcal{F}}$ is saturated. By Lemma 6.4(b), $\mathcal{H}$ contains all $\widetilde{\mathcal{F}}$-quasicentric subgroups of $\widetilde{S}$ which contain $A$, and in particular, all $\widetilde{\mathcal{F}}$-centric subgroups (since every $\widetilde{\mathcal{F}}$-centric subgroup of $\widetilde{S}$ must contain $A$ ). Since $\widetilde{\mathcal{F}}$ is $\mathcal{H}$-generated by construction, it remains only to prove condition (a) in Proposition 6.6.

Fix some $\widetilde{P}=\tau^{-1}(P)$ in $\mathcal{H}$, and let $\varphi \in \operatorname{Aut}_{\widetilde{\mathcal{F}}}(\widetilde{P})$ be such that $\tau_{*}(\varphi)=\operatorname{Id}_{P}$. Choose $(f, a) \in \widetilde{\pi}^{-1}(\varphi)$; thus

$$
\varphi=\widetilde{\pi}(f, a) \in \operatorname{Ker}\left[\operatorname{Aut}_{\widetilde{\mathcal{F}}}(\widetilde{P}) \longrightarrow \operatorname{Aut}_{\mathcal{F}}(P)\right] .
$$

Then $\pi(f)=\operatorname{Id}_{P}$, so $f=\delta_{P}(q)$ for some $q \in C_{S}(P)$, and $(f, a)=\delta_{\widetilde{P}}(q, c)$ for some $c \in A$. Since $\delta_{\widetilde{P}}$ is a monomorphism, the definition of $\varphi=\widetilde{\pi}(f, a)$ via (3) shows that $\varphi=\widetilde{\pi}(f, a)=\widetilde{\pi}\left(\delta_{\widetilde{P}}(q, c)\right)=c_{(q, c)}$, and thus that $\varphi \in \operatorname{Aut}_{\widetilde{S}}(\widetilde{P})$. Thus condition (a) in Proposition 6.6 holds, and this finishes the proof that $\widetilde{\mathcal{F}}$ is saturated.

$\widetilde{\mathcal{L}}^{q}$ is a quasicentric linking system associated to $\widetilde{\mathcal{F}}$ : The distinguished monomorphisms $\delta_{\widetilde{P}}$, for $\widetilde{P} \in \mathrm{Ob}\left(\widetilde{\mathcal{L}}^{q}\right)$, were chosen so as to satisfy $(\mathrm{D})_{q}$, and this was independent of the choice of inclusion morphisms which lift the chosen inclusion morphisms in $\mathcal{L}^{q}$. Once the $\delta_{\widetilde{P}}$ were determined, then $\widetilde{\pi}$ was defined to satisfy $(\mathrm{C})_{q}$, and $\widetilde{\mathcal{F}}$ was defined as the category generated by $\operatorname{Im}(\widetilde{\pi})$ and restrictions. Thus all of these structures were uniquely determined by the starting data. Axiom (B) ${ }_{q}$ follows from $(\mathrm{C})_{q}$ by Lemma 1.10 .

It remains only to prove $(\mathrm{A})_{q}$. We have already seen that the functor $\widetilde{\pi}$ is surjective on all morphism sets. Also, since $C_{\widetilde{S}}(P)=C_{\widetilde{S}}(P A)$ for all $P \leq \widetilde{S}$, it suffices to prove $(\mathrm{A})_{q}$ for morphisms between subgroups $\widetilde{P}=\tau^{-1}(P)$ and $\widetilde{Q}=$ $\tau^{-1}(Q)$ containing $A$. By construction, $C_{\widetilde{S}}(\widetilde{P})$ acts freely on each morphism set $\operatorname{Mor}_{\widetilde{\mathcal{L}}^{q}}(\widetilde{P}, \widetilde{Q})$, and it remains to show that if $\widetilde{P}$ is fully centralized, then $\widetilde{\pi}_{\widetilde{P}, \widetilde{Q}}$ is the orbit map of this action. As in the proof of Theorem 6.8(a), it suffices to do this when $\widetilde{P}$ and $P$ are fully normalized. 
Fix two morphisms $(f, a)$ and $(g, b)$ from $\widetilde{P}$ to $\widetilde{Q}$ such that $\widetilde{\pi}(f, a)=\widetilde{\pi}(g, b)$. Then $\pi(f)=\pi(g)$, so $g=f \circ \delta_{P}(x)$ for some $x \in C_{S}(P)$, and

$$
(g, b)=(f, a) \circ\left(\delta_{P}(x), b-a-\omega\left(\delta_{P}(x), f\right)\right)=(f, a) \circ \delta_{\tilde{P}}(x, c),
$$

where $c=b-a-\omega\left(\delta_{P}(x), f\right)-\left(\omega\left(\iota_{P}, \delta_{S}(x)\right)-\omega\left(\delta_{P}(x), \iota_{P}\right)\right)$. Also, $\widetilde{\pi}\left(\delta_{\widetilde{P}}(x, c)\right)=\operatorname{Id}_{\widetilde{P}}$ implies (via (3)) that $(x, c)$ commutes with all elements of $\widetilde{P}$, so $(x, c) \in C_{\widetilde{S}}(\widetilde{P})$, and this finishes the argument.

We now want to relate the obstruction theory for central extensions of linking systems with kernel $A$ to those for central extensions of $p$-groups, and to those for principal fibrations with fiber $B A$. As a consequence of this, we will show (in Theorem [6.13) that when appropriate restrictions are added, these three types of extensions are equivalent.

Given a central extension $\widetilde{\mathcal{F}}$ of $\mathcal{F}$ by the central subgroup $A$, there is an induced central extension $1 \rightarrow A \rightarrow \widetilde{S} \rightarrow S \rightarrow 1$ of Sylow subgroups. Restriction to subgroups $P \leq S$ produces corresponding central extensions $1 \rightarrow A \rightarrow \widetilde{P} \rightarrow P \rightarrow 1$. The homology classes of these central extensions are all compatible with morphisms

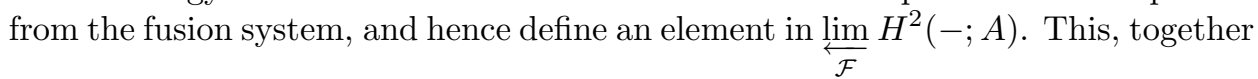
with notation already used in [BLO2, motivates the following definition:

Definition 6.11. For any saturated fusion system over a $p$-group $S$, and any finite abelian $p$-group $A$, define

$$
H^{*}(\mathcal{F} ; A)=\underbrace{\lim _{F}}_{\mathcal{F}} H^{*}(-; A) \cong \underbrace{\lim _{c}}_{\mathcal{F} c} H^{*}(-; A) .
$$

The following lemma describes the relation between the cohomology of $\mathcal{F}$ and the cohomology of the geometric realization of any linking system associated to $\mathcal{F}$.

Lemma 6.12. For any $p$-local finite group $(S, \mathcal{F}, \mathcal{L})$, and any finite abelian p-group $A$, the natural homomorphism

$$
H^{*}(|\mathcal{L}| ; A) \longrightarrow H^{*}(\mathcal{F} ; A)
$$

induced by the inclusion of $B S$ into $|\mathcal{L}|$, is an isomorphism. Furthermore, there are natural isomorphisms

$$
{\underset{\lim ^{q}}{ }}^{2}(A) \cong H^{2}\left(\left|\mathcal{L}^{q}\right| ; A\right) \cong H^{2}(|\mathcal{L}| ; A)
$$

Proof. The second isomorphism in (2) follows from Proposition 1.12(a). The first isomorphism holds for higher limits of any constant functor over any small, discrete category $\mathcal{C}$, since both groups are cohomology groups of the same cochain complex

$$
0 \longrightarrow \prod_{c} A \longrightarrow \prod_{c_{0} \rightarrow c_{1}} A \longrightarrow \prod_{c_{0} \rightarrow c_{1} \rightarrow c_{2}} A \longrightarrow \cdots .
$$

This cochain complex for higher limits is shown in GZ, Appendix II, Proposition 3.3] (applied with $\mathcal{M}=A \mathrm{~b}^{\mathrm{op}}$ ).

To prove the isomorphism (1), it suffices to consider the case where $A=\mathbb{Z} / p^{n}$ for some $n$. This was shown in BLO2, Theorem 5.8] when $A=\mathbb{Z} / p$, so we can assume that $n \geq 2$, and that the lemma holds when $A=\mathbb{Z} / p^{n-1}$. Consider the 
following diagram of Bockstein exact sequences:

(1)

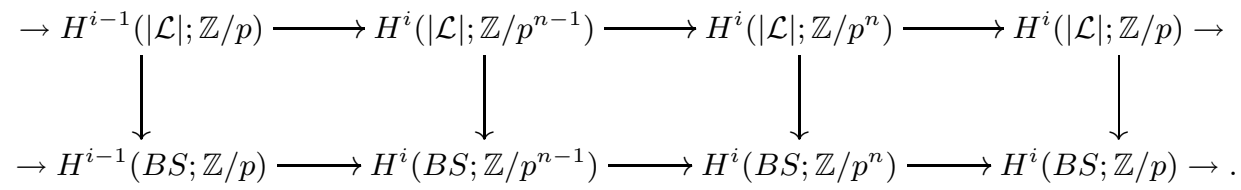

We claim that the bottom row restricts to an exact sequence of groups $H^{*}(\mathcal{F} ;-)$. Once this is shown, the result follows by the 5-lemma.

By [BLO2, Proposition 5.5], there is a certain $(S, S)$-biset $\Omega$ which induces, via a sum of composites of transfer maps and maps induced by homomorphisms, an idempotent endomorphism of $H^{*}(B S ; \mathbb{Z} / p)$ whose image is $H^{*}(\mathcal{F} ; \mathbb{Z} / p)$. This biset $\Omega$ also induces endomorphisms $[\Omega]$ of $H^{*}\left(B S ; \mathbb{Z} / p^{n}\right)$ and $H^{*}\left(B S ; \mathbb{Z} / p^{n-1}\right)$ which commute with the bottom row in $(1)$, since any exact sequence induced by a short exact sequence of coefficient groups will commute with transfer maps and maps induced by homomorphisms. The same argument as that used in the proof of BLO2, Proposition 5.5] shows that in all of these cases, $\operatorname{Im}([\Omega])=H^{*}(\mathcal{F} ;-)$, and the restriction of $[\Omega]$ to its image is multiplication by $|\Omega| /|S| \in 1+p \mathbb{Z}$. Thus the sequence of the $H^{*}(\mathcal{F} ;-)$ splits as a direct summand of the bottom row in $(1)$, and hence is exact.

We can now collect the results about central extensions of $(S, \mathcal{F}, \mathcal{L})$ in the following theorem, which is the analog for $p$-local finite groups of the classical classification of central extensions of groups.

Theorem 6.13. Let $(S, \mathcal{F}, \mathcal{L})$ be a p-local finite group. For each finite abelian p-group $A$, the following three sets are in one-to-one correspondence:

(a) equivalence classes of central extensions of $(S, \mathcal{F}, \mathcal{L})$ by $A$;

(b) equivalence classes of principal fibrations $B A \rightarrow X \rightarrow|\mathcal{L}|_{p}^{\wedge}$; and

(c) isomorphism classes of central extensions $1 \rightarrow A \rightarrow \widetilde{S} \rightarrow^{\tau} S \rightarrow 1$ for which each morphism $\varphi \in \operatorname{Hom}_{\mathcal{F}}(P, Q)$ lifts to some $\widetilde{\varphi} \in \operatorname{Hom}\left(\tau^{-1}(P), \tau^{-1}(Q)\right)$.

The equivalence between the first two is induced by taking classifying spaces, and the equivalence between (a) and (c) is induced by restriction to the underlying p-group. These sets are all in natural one-to-one correspondence with

$$
{\underset{\lim }{\mathcal{L}}}^{2}(A) \cong H^{2}(|\mathcal{L}| ; A) \cong H^{2}(\mathcal{F} ; A) \text {. }
$$

Proof. The three groups in (1) are isomorphic by Lemma 6.12, By Proposition 6.10. central extensions of $\mathcal{L}$ are classified by $\underset{\mathcal{L}}{\lim ^{2}}(A)$. Principal fibrations over $|\mathcal{L}|_{p}^{\wedge}$ with fiber $B A$ are classified by

$$
\left[|\mathcal{L}|_{p}^{\wedge}, B(B A)\right] \cong H^{2}\left(|\mathcal{L}|_{p}^{\wedge} ; A\right) \cong H^{2}(|\mathcal{L}| ; A),
$$

where the second isomorphism holds since $|\mathcal{L}|$ is $p$-good ([BLO2, Proposition 1.12]) and $A$ is an abelian $p$-group. Central extensions of $S$ by $A$ are classified by $H^{2}(S ; A)$, and the central extension satisfies the condition in (c) if and only if the corresponding element of $H^{2}(S ; A)$ extends to an element in the inverse limit $H^{2}(\mathcal{F} ; A)$.

A central extension of $p$-local finite groups induces a principal fibration of classifying spaces by Theorem 6.8(c), and this principal fibration restricts to the principal fibration over $B S$ of classifying spaces of $p$-groups. Thus, if the fibration 
over $|\mathcal{L}|$ is classified by $\chi \in H^{2}(|\mathcal{L}| ; A)$, then the fibration over $B S$ is classified by the restriction of $\chi$ to $H^{2}(B S ; A)$. It is well known that the invariant in $H^{2}(S ; A)=H^{2}(B S ; A)$ for a central extension of $S$ by $A$ is the same as that which describes the principal fibration over $B S$ with fiber $B A$ (see AM, Lemma IV.1.12]). Since $H^{2}(|\mathcal{L}| ; A)$ injects into $H^{2}(\mathcal{F} ; A)$ (Lemma 6.12), this shows that $\chi$ is also the class of the 2-cocycle which defines the extension of categories. So the map between the sets in (a) and (b) defined by taking geometric realization is equal to the bijection defined by the obstruction theory.

Since the isomorphism ${\underset{\mathcal{L}}{\mathcal{L}}}^{2}(A) \cong H^{2}(\mathcal{F} ; A)$ is defined by restriction to $S$ (as a group of automorphisms in $\mathcal{L}$ ), the bijection between $(\mathrm{a})$ and $(\mathrm{c})$ induced by the bijection of obstruction groups is the same as that induced by restriction to $S$.

The following corollary shows that all minimal examples of "exotic" fusion systems have trivial center.

Corollary 6.14. Let $\mathcal{F}$ be a saturated fusion system over a p-group $S$, and assume there is a nontrivial subgroup $1 \neq A \leq Z(S)$ which is central in $\mathcal{F}$. Then $\mathcal{F}$ is the fusion system of some finite group if and only if $\mathcal{F} / A$ is.

Proof. Assume $\mathcal{F}$ is the fusion system of the finite group $G$, with $S \in \operatorname{Syl}_{p}(G)$. By assumption $(A$ is central in $\mathcal{F}$ ), each morphism in $\mathcal{F}$ extends to a morphism between subgroups containing $A$ which is the identity on $A$. Hence $\mathcal{F}$ is also the fusion system of $C_{G}(A)$ over $S$, and so $\mathcal{F} / A$ is the fusion system of $C_{G}(A) / A$.

It remains to prove the converse. Assume $\mathcal{F} / A$ is isomorphic to the fusion system of the finite group $\bar{G}$, and identify $S / A$ with a Sylow $p$-subgroup of $\bar{G}$. Since by Lemma 6.12 $H^{2}(\mathcal{F} / A ; A) \cong H^{2}(B \bar{G} ; A)$ and $\mathrm{A}$ is $\mathcal{F}$-central, the cocycle classifying the extension is in $H^{2}(B \bar{G} ; A)$, and hence there is an extension of finite groups

$$
1 \longrightarrow A \longrightarrow G \stackrel{\tau}{\longrightarrow} \bar{G} \longrightarrow 1
$$

with the same obstruction invariant as the extension $\mathcal{F} \rightarrow \mathcal{F} / A$. In particular, we can identify $S=\tau^{-1}(S / A) \in \operatorname{Syl}_{p}(G)$, and $\bar{G}=G / A$.

We will prove the following two statements:

(a) $\mathcal{F}$ has an associated centric linking system $\mathcal{L}$; and

(b) $\mathcal{L}_{S / A}^{c}(G / A)$ is the unique centric linking system associated to the fusion system $\mathcal{F} / A=\mathcal{F}_{S / A}(G / A)$.

Once these have been shown, then they imply that

$$
\left(S / A, \mathcal{F} / A,(\mathcal{L} / A)^{c}\right) \cong\left(S / A, \mathcal{F}_{S / A}(G / A), \mathcal{L}_{S / A}^{c}(G / A)\right)
$$

as $p$-local finite groups. Hence $(S, \mathcal{F}, \mathcal{L}) \cong\left(S, \mathcal{F}_{S}(G), \mathcal{L}_{S}^{c}(G)\right)$ as $p$-local finite groups by Theorem 6.13, and thus $\mathcal{F}$ is the fusion system of $G$.

It remains to prove (a) and (b). Let

$$
\left.\mathcal{Z}_{\mathcal{F}}: \mathcal{O}^{c}(\mathcal{F}) \longrightarrow \mathbb{Z}_{(p)^{-} \bmod } \quad \text { and } \quad \mathcal{Z}_{G}: \mathcal{O}_{S}^{c}(G) \longrightarrow \mathbb{Z}_{(p)}\right)^{-\bmod }
$$

be the categories and functors of Definition [1.7 By [01, Lemma 2.1], $\mathcal{Z}_{G}$ can also be regarded as a functor on $\mathcal{O}^{c}\left(\mathcal{F}_{S}(G)\right)$, and

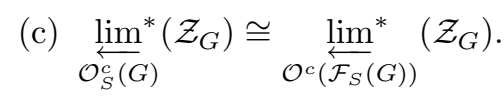


By [BLO2, Proposition 3.1], the existence and uniqueness of a centric linking system depends on the vanishing of certain obstruction classes: the obstruction to existence lies in $\underset{\mathcal{O}_{S}^{c}(G)}{\lim ^{3}}\left(\mathcal{Z}_{\mathcal{F}}\right)$ and the obstruction to uniqueness in ${\underset{\mathcal{O}_{S}^{c}(G)}{\lim }}^{2}\left(\mathcal{Z}_{\mathcal{F}}\right)$. Thus (b) follows from [BLO2, Proposition 3.1] and (c), once we know that $\lim ^{2}\left(\mathcal{Z}_{G / A}\right)=$ 0 ; and this is shown in [01, Theorem A] (if $p$ is odd) or [O2, Theorem $\mathrm{A}$ ] (if $p=2$ ).

It remains to prove point (a), and we will do this by showing that

$$
{\underset{\mathcal{O}}{\mathcal{O}^{c}(\mathcal{F})}}^{3}\left(\mathcal{Z}_{\mathcal{F}}\right) \cong{\underset{\mathcal{O}}{\check{S}(G)}}_{\lim ^{3}}\left(\mathcal{Z}_{G}\right)=0
$$

The last equality follows from [01, Theorem A] or [O2, Theorem A] again, so it remains only to prove the isomorphism.

Let $\mathcal{H}$ be the set of subgroups $P \leq S$ containing $A$ such that $P / A$ is $\mathcal{F} / A$ centric; or equivalently, $p$-centric in $G / A$. Let $\mathcal{O}^{\mathcal{H}}(\mathcal{F}) \subseteq \mathcal{O}^{c}(\mathcal{F})$ and $\mathcal{O}^{\mathcal{H}}\left(\mathcal{F}_{S}(G)\right) \subseteq$ $\mathcal{O}^{c}\left(\mathcal{F}_{S}(G)\right)$ be the full subcategories with object sets $\mathcal{H}$.

We claim that

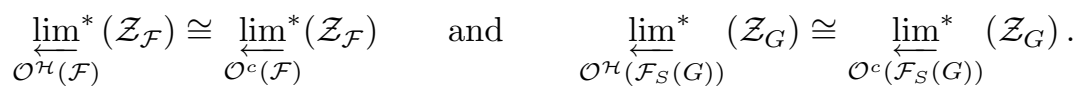

If $P \leq S$ is $\mathcal{F}$-centric but not in $\mathcal{H}$, then there is $x \in N_{S}(P) \backslash P$ such that $c_{x} \in \operatorname{Aut}_{\mathcal{F}}(P)$ is the identity on $P / A$ and on $A$, but is not in $\operatorname{Inn}(P)$. Thus $1 \neq\left[c_{x}\right] \in O^{p}\left(\operatorname{Out}_{\mathcal{F}}(P)\right)$, and so $P$ is not $\mathcal{F}$-radical. By [BLO2, Proposition 3.2], if $F: \mathcal{O}^{c}(\mathcal{F})^{\text {op }} \rightarrow \mathbb{Z}_{(p)}$-mod is any functor which vanishes except on the conjugacy class of $P$, then $\lim ^{*}(F) \cong \Lambda^{*}\left(\operatorname{Out}_{\mathcal{F}}(P) ; F(P)\right)$, where $\Lambda^{*}$ is a certain functor defined in [JMO, §5]. By [JMO, Proposition 6.1(ii)], $\Lambda^{*}\left(\operatorname{Out}_{\mathcal{F}}(P) ; F(P)\right)=0$ for any $F$, since $O_{p}\left(\operatorname{Out}_{\mathcal{F}}(P)\right) \neq 1$. From the long exact sequences of higher limits induced by an extension of functors, it now follows that $\lim _{\leftarrow}^{*}(F)=0$ for any functor $F$ on $\mathcal{O}^{c}(\mathcal{F})$ which vanishes on $\mathcal{O}^{\mathcal{H}}(\mathcal{F})$; and this proves the first isomorphism in $(2)$. The second isomorphism follows by a similar argument.

The natural surjection of $\mathcal{F}$ onto $\mathcal{F} / A$ induces isomorphisms of categories

$$
\mathcal{O}^{\mathcal{H}}(\mathcal{F}) \cong \mathcal{O}^{c}(\mathcal{F} / A) \cong \mathcal{O}^{\mathcal{H}}\left(\mathcal{F}_{S}(G)\right) \text {. }
$$

By the definition of $\mathcal{H}$, we clearly have bijections between the sets of objects in these categories, so it remains only to compare morphism sets. The result follows from Lemma 6.5. (a) for sets of morphisms between pairs of fully normalized subgroups, and the general case follows since every object is isomorphic to one which is fully normalized.

We next claim that for all $i>0$,

$$
\text { (4) } \lim _{\mathcal{O}^{\leftarrow}(\mathcal{F})}^{i}\left(\mathcal{Z}_{\mathcal{F}}\right) \cong \lim _{\mathcal{O}^{c}(\mathcal{F} / A)}^{i}\left(\mathcal{Z}_{\mathcal{F}} / A\right) \quad \text { and } \quad \underbrace{i}_{\mathcal{O}^{\mathcal{H}}\left(\dot{\mathcal{F}}_{S}(G)\right)}\left(\mathcal{Z}_{G}\right) \cong \underbrace{\lim ^{i}}_{\mathcal{O}^{c}(\mathcal{F} / A)}\left(\mathcal{Z}_{G} / A\right) \text {. }
$$

To show this, by (3), together with the long exact sequence of higher limits induced by the short exact sequence of functors

$$
1 \rightarrow A \longrightarrow \mathcal{Z}_{\mathcal{F}} \longrightarrow \mathcal{Z}_{\mathcal{F}} / A \rightarrow 1,
$$

we need only show that $\underset{\mathcal{O}^{c}(\mathcal{F} / A)}{\lim ^{i}}(A)=0$ for all $i>0$. Here, $A$ denotes the constant functor on $\mathcal{O}^{c}(\mathcal{F} / A)$ which sends all objects to $A$ and all morphisms to $\operatorname{Id}_{A}$. For each $P \in \mathcal{H}$, let $F_{A, P}$ be the functor on $\mathcal{O}^{c}(\mathcal{F} / A)$ where $F_{A, P}\left(P^{\prime} / A\right)=A$ if 
$P^{\prime}$ is $\mathcal{F}$-conjugate to $P$ and $F_{A, P}\left(P^{\prime} / A\right)=0$ otherwise; and which sends isomorphisms between subgroups conjugate to $P$ to $\mathrm{Id}_{A}$. By [BLO2, Proposition 3.2], $\lim ^{*}\left(F_{A, P}\right) \cong \Lambda^{*}\left(\operatorname{Out}_{\mathcal{F} / A}(P / A) ; A\right)$. Also, $\Lambda^{i}\left(\operatorname{Out}_{\mathcal{F} / A}(P / A) ; A\right)=0$ for $i>0$ by [JMO, Proposition 6.1(i,ii)], since the action of $\operatorname{Out}_{\mathcal{F} / A}(P / A)$ on $A$ is trivial. From the long exact sequences of higher limits induced by an extension of functors, we now see that $\lim ^{i}(A)=0$ for all $i>0$.

Finally, we claim that

$$
\mathcal{Z}_{\mathcal{F}} / A \cong \mathcal{Z}_{G} / A
$$

as functors on $\mathcal{O}^{c}(\mathcal{F} / A)$ under the identifications in (3). To see this, note that for each $P,\left(\mathcal{Z}_{\mathcal{F}} / A\right)(P)=Z(P) / A=\left(\mathcal{Z}_{G} / A\right)(P)$; and since these are both identified as subgroups of $P / A$, any morphism in $\mathcal{O}^{c}(\mathcal{F} / A)$ from $P / A$ to $Q / A$ induces the same map (under the two functors) from $Z(Q) / A$ to $Z(P) / A$. (This argument does not apply to prove that $\mathcal{Z}_{\mathcal{F}} \cong \mathcal{Z}_{G}$. These two functors send each object to the same subgroup of $S$, but we do not know that they send each morphism to the same homomorphism between the subgroups.)

Thus by (2), (4), and (5), for all $i>0$,

$$
\begin{aligned}
& \lim _{\mathcal{O}^{c}(\mathcal{F})}^{i}\left(\mathcal{Z}_{\mathcal{F}}\right) \cong \lim _{\mathcal{O}^{\mathcal{H}(\mathcal{F})}}^{i}\left(\mathcal{Z}_{\mathcal{F}}\right) \cong \lim _{\mathcal{O}^{c}(\mathcal{F} / A)}^{i}\left(\mathcal{Z}_{\mathcal{F}} / A\right)
\end{aligned}
$$

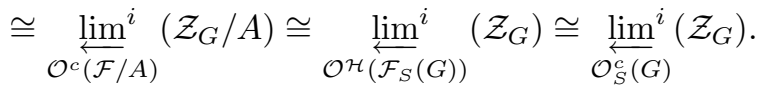

This finishes the proof of (1), and hence finishes the proof of the corollary.

\section{REFERENCES}

[AM] A. Adem \& J. Milgram, Cohomology of finite groups, Springer-Verlag (1994) MR.1317096 (96f:20082)

[Al] J. Alperin, Sylow intersections and fusion, J. Algebra 6 (1967), 222-241 MR0215913 (35:6748)

[BK] P. Bousfield \& D. Kan, Homotopy limits, completions, and localizations, Lecture notes in math. 304, Springer-Verlag (1972) MR0365573 (51:1825)

[5A1] C. Broto, N. Castellana, J. Grodal, R. Levi and B. Oliver, Subgroup families controlling p-local finite groups, Proc. London Math. Soc. 91 (2005), 325-354 MR2167090

[BLO1] C. Broto, R. Levi, \& B. Oliver, Homotopy equivalences of $p$-completed classifying spaces of finite groups, Invent. math. 151 (2003), 611-664 MR1961340(2004c:55031)

[BLO2] C. Broto, R. Levi, \& B. Oliver, The homotopy theory of fusion systems, J. Amer. Math. Soc. 16 (2003), 779-856 MR1992826 (2004k:55016)

[DKS] W. Dwyer, D. Kan, \& J. Smith, Towers of fibrations and homotopical wreath products, J. Pure Appl. Algebra 56 (1989), 9-28 MR.0974710 (89k:55009)

[GZ] P. Gabriel \& M. Zisman, Calculus of fractions and homotopy theory, Springer-Verlag (1967) MR.0210125 (35:1019)

[Gl] G. Glauberman, Central elements in core-free groups, J. Algebra 4 (1966), 403-420 MR0202822 (34:2681)

[GJ] P. Goerss \& R. Jardine, Simplicial homotopy theory, Birkhäuser (1999) MR.1711612 (2001d:55012)

[Go] D. Gorenstein, Finite groups, Harper \& Row (1968) MR0231903 (38:229)

[Hf] G. Hoff, Cohomologies et extensions de categories, Math. Scand. 74 (1994), 191-207. MR.1298361 (95k:18012)

[JMO] S. Jackowski, J. McClure, \& B. Oliver, Homotopy classification of self-maps of $B G$ via $G$-actions, Annals of Math. 135 (1992), 184-270

[May] P. May, Simplicial objects in algebraic topology, Van Nostrand (1967) MR0222892 $(36: 5942)$ 
[O1] B. Oliver, Equivalences of classifying spaces completed at odd primes, Math. Proc. Camb. Phil. Soc. 137 (2004), 321-347 MR2092063 (2006g:55010)

[O2] B. Oliver, Equivalences of classifying spaces completed at the prime two, Amer. Math. Soc. Memoirs 848 (2006) MR2203209 (2007c:55014)

[Pu1] Ll. Puig, Unpublished notes (ca. 1990)

[Pu2] Ll. Puig, The hyperfocal subalgebra of a block, Invent. math. 141 (2000), 365-397 MR 1775217 (2001h:20012)

[Pu3] L. Puig, Full Frobenius systems and their localizing categories, preprint (2001)

[St] R. Stancu, Equivalent definitions of fusion systems, preprint

[Suz2] M. Suzuki, Group theory II, Springer-Verlag (1986) MR0815926 (87e:20001)

Departament de Matemàtiques, Universitat Autònoma de Barcelona, E-08193 BelLATERra, Spain

E-mail address: broto@mat.uab.es

Departament de Matemàtiques, Universitat Autònoma de Barcelona, E-08193 BelLATERRA, SPAIN

E-mail address: natalia@mat.uab.es

Department of Mathematics, University of Chicago, Chicago, Illinois 60637

E-mail address: jg@math.uchicago.edu

Current address: Department of Mathematical Sciences, University of Copenhagen, Universitetsparken 5, DK-2100 København Ø, Denmark

E-mail address: jg@math.ku.dk

Department of Mathematical Sciences, University of Aberdeen, Meston Building 339, Aberdeen AB24 3UE, United Kingdom

E-mail address: ran@maths.abdn.ac.uk

LAGa, Institut Galilée, Av. J-B Clément, 93430 Villetaneuse, France

E-mail address: bob@math.univ-paris13.fr 